\title{
Regulation of skeletal muscle recovery
}

Citation for published version (APA):

Kneppers, A. (2018). Regulation of skeletal muscle recovery: Implications for COPD. [Doctoral Thesis, Maastricht University]. ProefschriftMaken Maastricht. https://doi.org/10.26481/dis.20181221ak

Document status and date:

Published: 01/01/2018

DOI:

10.26481/dis.20181221ak

Document Version:

Publisher's PDF, also known as Version of record

\section{Please check the document version of this publication:}

- A submitted manuscript is the version of the article upon submission and before peer-review. There can be important differences between the submitted version and the official published version of record.

People interested in the research are advised to contact the author for the final version of the publication, or visit the DOI to the publisher's website.

- The final author version and the galley proof are versions of the publication after peer review.

- The final published version features the final layout of the paper including the volume, issue and page numbers.

Link to publication

\footnotetext{
General rights rights.

- You may freely distribute the URL identifying the publication in the public portal. please follow below link for the End User Agreement:

www.umlib.nl/taverne-license

Take down policy

If you believe that this document breaches copyright please contact us at:

repository@maastrichtuniversity.nl

providing details and we will investigate your claim.
}

Copyright and moral rights for the publications made accessible in the public portal are retained by the authors and/or other copyright owners and it is a condition of accessing publications that users recognise and abide by the legal requirements associated with these

- Users may download and print one copy of any publication from the public portal for the purpose of private study or research.

- You may not further distribute the material or use it for any profit-making activity or commercial gain

If the publication is distributed under the terms of Article $25 \mathrm{fa}$ of the Dutch Copyright Act, indicated by the "Taverne" license above, 


\title{
REGULATION OF SKELETAL MUSCLE RECOVERY
}

IMPLICATIONS FOR COPD

\author{
DISSERTATION
}

by

Anna Elisabeth Maria Kneppers 


\section{Q}

(c) Copyright Anita Kneppers, Maastricht 2018

Cover design: Roy Haast

Layout: $\quad$ Anita Kneppers

Printed by: $\quad$ ProefschriftMaken | www.proefschriftmaken.nl

ISBN: $\quad$ 978-94-6380-168-3

The research described in this thesis was performed at the Department of Respiratory Medicine, in close collaboration with the Department of Human Biology, NUTRIM School of Nutrition and Translational Research in Metabolism at Maastricht University Medical Center+, Maastricht, the Netherlands. 


\title{
REGULATION OF SKELETAL MUSCLE RECOVERY
}

\section{IMPLICATIONS FOR COPD}

\author{
DISSERTATION \\ To obtain the degree of doctor at Maastricht University, \\ on the authority of the Rector Magnificus, Prof. dr. Rianne M. Letschert \\ in accordance with the decision of the Board of Deans, \\ to be defended in public on Friday 21 December 2018, at 10:00 hours \\ by
}

\section{Anna Elisabeth Maria Kneppers}

born in Valkenburg aan de Geul, the Netherlands, 30 June 1990 


\section{Promotor}

Prof. dr. ir. A.M.W.J Schols

\section{Co-promotors}

Dr. R.C.J. Langen

Dr. L.B. Verdijk

\section{Assessment Committee}

Prof. dr. M.K.C. Hesselink (Chair)

Prof. dr. B.L.M. Schroen

Prof. dr. ir. A.H. Kersten (WUR, Wageningen)

Prof. dr. M. Sandri (UNIPD, Padova, Italy)

Dr. F.M.E. Franssen 


\section{CONTENTS}

$\begin{array}{llr}\text { Chapter } 1 & \text { General introduction } & 7\end{array}$

Chapter 2 Cachexia in chronic obstructive pulmonary disease: new insights and therapeutic perspective

Chapter 3 Increased myogenic and protein turnover signaling in skeletal muscle of chronic obstructive pulmonary disease patients with sarcopenia

Chapter 4 Coordinated regulation of skeletal muscle mass and metabolic plasticity during recovery from disuse

Chapter 5 Distinct skeletal muscle molecular responses to pulmonary rehabiliation in COPD: a cluster analysis

Chapter 6 A novel in vitro model for the assessment of postnatal myonuclear accretion

Chapter 7 Preliminary report: Increased myonuclear accretion during recovery from metabolic stress

Chapter 8 General discussion

Summary

Samenvatting

Valorization

Acknowledgments

Curriculum Vitae 

GENERAL INTRODUCTION 


\section{CHAPTER 1}

Skeletal muscle is essential for the generation of forces for postural support and physical functioning. However, skeletal muscle is not just a locomotor organ, but also an important site for control of metabolism [1]. These functions are supported by the muscles' biomechanical and morphological makeup, which are tightly regulated, and can rapidly adapt to alterations in mechanical and metabolic demand - referred to as skeletal muscle plasticity. A well-studied display of skeletal muscle plasticity is the gain in muscle mass and muscle fiber cross-sectional-area (i.e., hypertrophy) upon resistance-type exercise [2]. Conversely, reduced mechanical loading and prolonged starvation lead to the loss of skeletal muscle mass and muscle fiber cross-sectional area (i.e., atrophy) [3, 4]. Both hypertrophy and atrophy are part of normal skeletal muscle plasticity, and may occur in various situations throughout life. Importantly, being the resultant of these processes, total skeletal muscle mass is a strong predictor of survival $[5,6]$, and a robust control of muscle mass is critical for the maintenance of whole-body homeostasis and health [7].

A decreased muscle mass and function (i.e., muscle wasting) is more prevalent with advancing age, and in patients with chronic obstructive pulmonary disease (COPD) compared to age-matched controls [8]. This muscle pathology is generally ascribed to either chronic catabolism or frequent acute catabolic events, although to date, limited longitudinal studies are available to support this hypothesis. Conversely, a potential role for impaired recovery after muscle atrophy is both underappreciated and understudied. In this thesis, we aim to provide further insight in the regulation of skeletal muscle plasticity using a translational research approach, with a specific focus on recovery after atrophy and potential implications for COPD-induced skeletal muscle pathology.

\section{Protein turnover regulation of skeletal muscle mass plasticity}

Plasticity of skeletal muscle mass is primarily regulated by the balance between muscle protein synthesis and muscle protein degradation over time (i.e., protein turnover) (Figure 1) [2]. Whereas a constant muscle mass is maintained due to a dynamic balance between these processes, hypertrophy occurs when muscle protein synthesis persistently exceeds muscle protein degradation, and conversely, atrophy occurs when protein degradation persistently exceeds protein synthesis.

The muscle protein turnover balance is regulated by sequential expression programs that orchestrate protein synthesis and degradation pathways, coordinated by a complex biological network of intracellular signaling mechanisms, as reviewed by e.g. Bilodeau et al. and Langen et al. $[9,10]$. Signals that act as anabolic or catabolic 
A
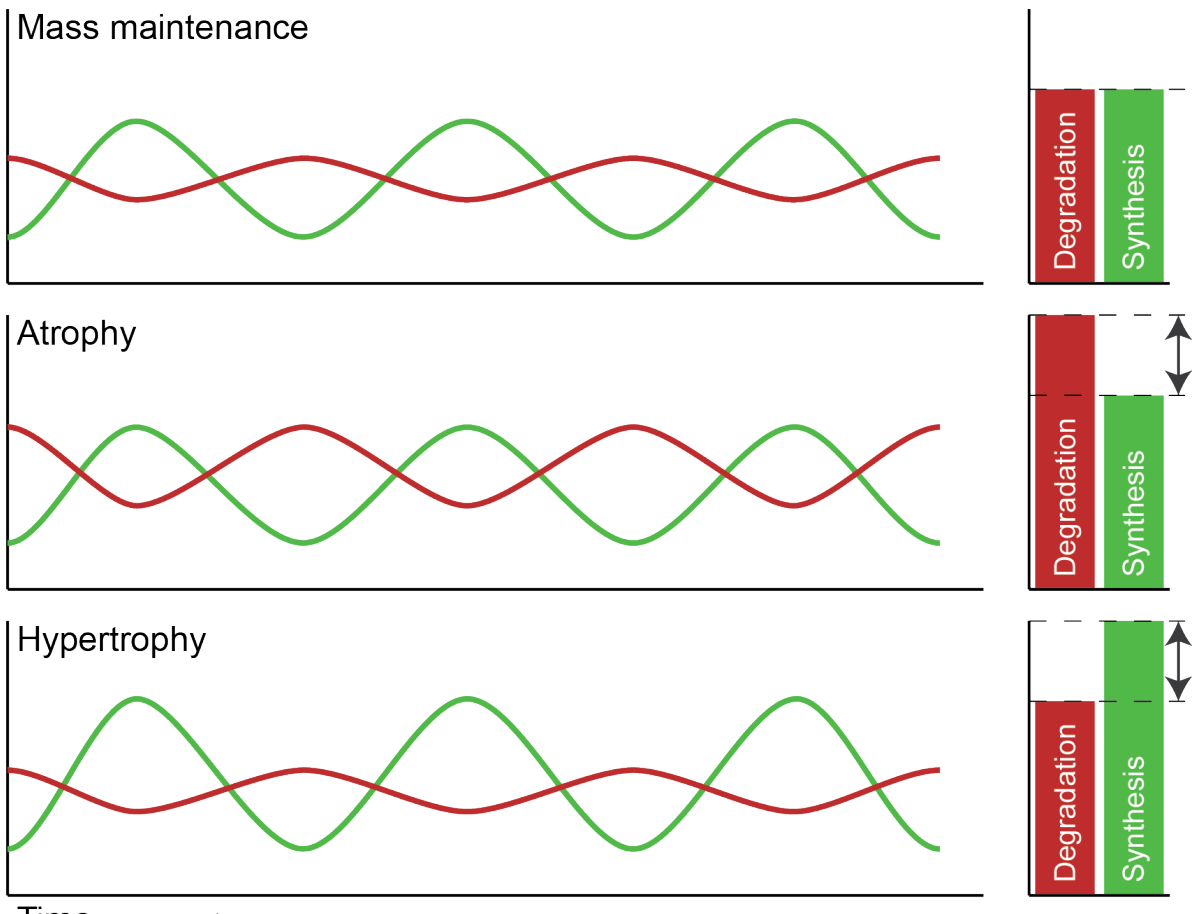

Time

\section{B}
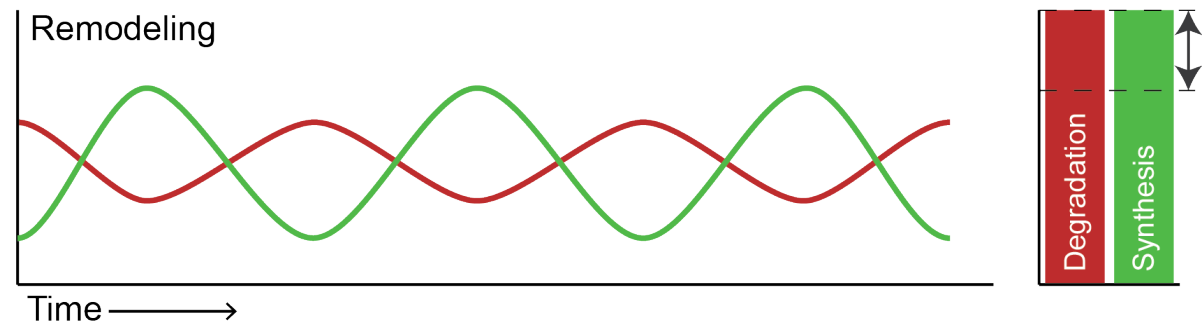

Figure 1. | Role of anabolic and catabolic processes in the regulation of muscle mass and quality. A. Schematic presentation of muscle mass regulation, dictated by the difference between protein synthesis and degradation, and coordinated by skeletal muscle protein synthesis and degradation signaling. B. Schematic presentation of muscle quality regulation, dictated by the rate rather than the balance of protein turnover, and coordinated by skeletal muscle protein synthesis and protein degradation. Bars represent area under the curve.

triggers are integrated via AKT serine/threonine kinase 1 (AKT1), which coordinates the major protein turnover regulatory pathways; including protein translation, and the degradation of proteins via the autophagy-lysosome pathway (autophagy) and ubiquitin proteasome system (UPS) [11]. Specifically, anabolic factors signal through insulin-like growth factor 1 (IGF1)/phosphatidylinositol 3-kinase (PI3K)/AKT1, leading to 


\section{CHAPTER 1}

the activation of mechanistic target of rapamycin kinase (mTOR). Subsequently, mTOR phosphorylates and activates ribosomal protein S6 kinase B1 (RPS6KB1) and inhibits eukaryotic translation initiation factor 4E binding protein 1 (EIF4EBP1), resulting in the activation of protein translation $[12,13]$. Furthermore, anabolic signaling inhibits the induction of protein degradation by affecting both the autophagy-lysosome system (autophagy) and ubiquitin proteasome system (UPS). Specifically, mTOR phosphorylates unc-51-like kinase (ULK1) at ser757, thereby preventing activation of autophagy initiation [13,14]. Moreover, activated AKT phosphorylates Forkhead box O (FOXO), thereby preventing its nuclear translocation, and inhibiting the transcription of autophagy and UPS-related genes $[12,14]$. Conversely, catabolic signaling leads to the inhibition of protein translation, as well as the nuclear translocation of FOXO and nuclear factor KB (NF-KB), resulting in the transcription of autophagy- and UPSrelated genes $[12,14]$. Furthermore, a reduced AKT1/mTOR signaling and induced AMPK signaling lead to an increase in ULK1 activating phosphorylation at ser555, and subsequent induction of autophagy initiation [13]. Together, this shows a tight coordination between anabolic and catabolic pathways through shared critical nodes, including sensors of mechanical and metabolic activity [15, 16], which respond to alterations in contractile activity, energy status, nutrient availability, growth factors, and cytokines.

\section{Drivers of skeletal muscle atrophy}

Although often described in the context of pathological conditions, muscle atrophy is also part of a normal physiological adaptation to decreased mechanical and metabolic demand. Exemplary to this is disuse-induced atrophy, which acutely leads to an increase in UPS and autophagy signaling, and a decrease in protein synthesis signaling in skeletal muscle tissue [17-19]. Similarly, such catabolic signaling is induced during periods of amino acid deprivation [18]. However, although both disuse and an insufficient dietary protein intake may occur intermittently in young, healthy subjects, these conditions frequently persist in elderly and in patients with COPD [2023], leading to the progressive loss of muscle mass. Importantly, while muscle atrophy under these conditions may be part of a normal adaptation to reduced physical activity and low protein intake, long-term skeletal muscle disuse has been shown to reduce anabolic responsiveness $[24,25]$. Particularly in patients with COPD, sustained catabolic signaling is reinforced by a plethora of pathological factors, which include systemic inflammation and hypoxia [26-29]. These conditions further increase muscle protein breakdown, thereby skewing the dynamic muscle protein turnover balance towards a net degradation of muscle tissue. These adaptations are outside the normal range of skeletal muscle plasticity, and are associated with a reduced ability to restore 
skeletal muscle homeostasis [30].

\section{Cellular regulation of skeletal muscle mass recovery}

In muscle wasted, but otherwise healthy individuals, skeletal muscle homeostasis is regained upon the removal of catabolic triggers [31, 32]. However, for a normalization of muscle mass and function to occur, a period of excess protein synthesis is required. Indeed, upon skeletal muscle reloading after disuse atrophy, IGF1 mRNA expression and AKT1/mTOR signaling are transiently increased, while FOXO signaling and atrogene expression are reduced [33-37]. Skeletal muscle overload-training may further augment this response beyond the 'natural' reloading response, and has been shown to promote the recovery of muscle mass [35].

In addition to the anabolic shift in protein turnover balance, reloading and exercise training after muscle atrophy induce the accretion of myonuclei [38]. Due to the postmitotic nature of the syncytial muscle fibers, myonuclear accretion is accomplished by donation of satellite cell-derived myonuclei [39]. Skeletal muscle satellite cells are stem cells that contribute to skeletal muscle maintenance, growth, repair, and regeneration through their sequential proliferation, differentiation, and fusion with a muscle fiber - a process referred to as 'postnatal myogenesis' $[40,41]$ (Figure 2). In the adult, satellite cells are mitotically quiescent, and display limited gene transcription and translation [42]. However, upon external ques such as exercise and muscle injury, satellite cells become activated and proliferate to expand the precursor pool [38, 42, 43]. Subsequently, satellite cells either return to quiescence to replenish the satellite cell pool, or initiate the myogenic transcription program, and fuse to give rise to a new muscle fiber or donate their content to a pre-existing muscle fiber to contribute to muscle fiber (re)generation, repair, and growth $[42,43]$. The requirement of satellite cells for skeletal muscle hypertrophy and recovery has been clearly demonstrated in animal studies, which show that satellite cell depletion prevents sustained overload-induced muscle hypertrophy and recovery from muscle injury [39, 44-48]. Furthermore, the role of satellite cells in human skeletal muscle is demonstrated by the acute increase in satellite cell activation status and number after exercise, and the positive association between changes in muscle fiber size, satellite cell content, and myonuclear content after prolonged exercise training [38].

In both elderly and COPD patients, the negative consequences of muscle atrophy persist, potentially due to impaired skeletal muscle recovery [34, 49-51]. Indeed, although exercise training elicits overall beneficial effects, the anabolic response to exercise training is blunted in elderly and patients with COPD [52-54]. To overcome 


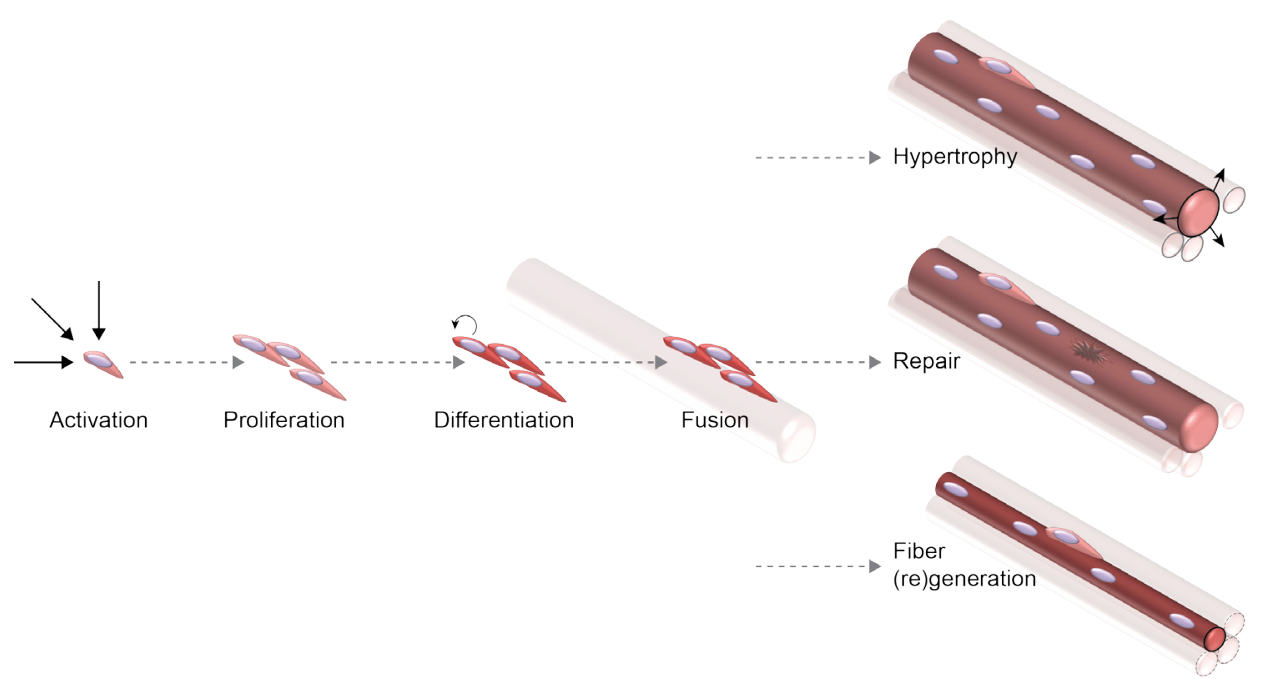

Figure 2. | Satellite cell function in the regulation of muscle mass, repair and regeneration. Upon external ques, satellite cells are activated and proliferate to expand the precursor pool. Subsequently, cells differentiate and fuse with a pre-existing muscle fiber to donate their nucleus to facilitate muscle fiber re-growth after atrophy or extensive muscle growth e.g. after resistance exercise (hypertrophy), or muscle fiber repair after damage or injury. In addition, differentiated satellite cells can fuse amongst each other to regenerate muscle after muscle fiber loss. Moreover, a subpopulation of satellite cells returns to quiescence, to maintain the precursor pool.

this, several anabolic stimuli have been proposed to aid the exercise training response, including anabolic steroid administration and nutritional supplementation [35, 5557]. However, these additional anabolic stimuli only provide marginal improvements in muscle function above the effect of exercise training alone [57, 58]. In part, this impairment in the functional response to anabolic stimuli may be due to systemic factors, particularly in the case of COPD. However, there is also ample evidence for skeletal muscle intrinsic alterations in both elderly and patients with COPD, with a central role for satellite cells $[59,60]$. Indeed, both elderly and COPD patients display a decrease in satellite cell number and/or function [60-63], which likely contributes to reduced skeletal muscle recovery and, as a consequence, the progressive loss of skeletal muscle mass.

\section{Cellular regulation of skeletal muscle quality}

Importantly, skeletal muscle pathology in patients with COPD is not just characterized by a change in muscle mass, but also by a change in the biochemical and structural makeup of the muscle. These changes include a shift in fiber type distribution, a decreased oxidative capacity, and mitochondrial dysfunction [64], which contribute to the loss of physical function. Furthermore, COPD is associated with changes in the extracellular environment, which similar to the observations in elderly, may include 
the accumulation of fibrotic tissue and intermuscular adipose tissue (IMAT), and the reduction of capillary density and number of neuromuscular junctions [65-67]. These qualitative alterations may be driven by COPD-associated factors such as hypoxia, inflammation and disuse [68-71], which are also implicated in the loss of muscle mass. Moreover, alterations in muscle quality may in itself contribute to the loss of muscle mass and thereby accelerate the progression of muscle pathology [72], suggesting that COPD-induced muscle pathology can be at least partially regarded as a model of accelerated aging.

Although anabolic stimuli such as hormone replacement therapies (e.g. androgens), dietary interventions (e.g. $\beta$-Alanine), and pharmaceuticals (e.g. PPARGC1A and PPIF inhibitors) have also been proposed as means to specifically counteract skeletal muscle qualitative alterations such as the loss of oxidative capacity and fat infiltration, these treatments seem to lack effectiveness [73]. In contrast, physical activity and exercise training were shown to improve muscle quality in both elderly and patients with COPD, depending on the exercise stimulus $[54,65,66,73]$. For example, exercise training has been shown to promote mitochondrial biogenesis and mitochondrial respiration $[65,74]$.

The plasticity of skeletal muscle quality upon altered external ques, by e.g. exercise training, comprises both the change in biochemical and morphological makeup, as well as the changes in the functionality of the cellular components. To facilitate this, both anabolic and catabolic processes are involved [75, 76], which interestingly, display a large overlap with the molecular mechanisms involved in the regulation of muscle mass (Figure 1). Indeed, it was shown that the catabolic pathways autophagy and mitophagy are essential in the mediation of skeletal muscle metabolic alterations upon recovery from muscle injury and in response to exercise training $[75,77]$. Furthermore, there are indications that autophagy could play a role in the change in fiber type composition upon exercise [78]. Interestingly, exercise-induced activation of autophagy even involves a response similar as to those triggers that are implicated in the induction of muscle atrophy (e.g. energy and nutrient deprivation [79]), although with exercise these originate intracellularly, and usually in the presence of an anabolic environment. In concert with catabolic signaling, exercises increases anabolic signaling though the AKT1/mTOR pathway [80]. Although this is known to promote protein synthesis, it also coordinates mitochondrial biogenesis and activity [81], reflecting an overlap in the protein turnover regulation of muscle mass and quality.

Similarly, satellite cells may have a role in the regulation of muscle quality in addition 


\section{CHAPTER 1}

to their role in muscle mass regulation. Indeed, it has been shown that satellite cells are not only activated upon damaging exercise, but also upon endurance exercise [82]. Recent evidence indicates a role for satellite cells in the regulation of the skeletal muscle quality by modulation of the muscle fiber microenvironment [83]. In addition, there are indications for interconnections between satellite cells and the muscle fiber protein turnover regulation of muscle fiber remodeling [84]. Interestingly, there is extensive evidence that alterations in skeletal muscle metabolism affect the activation and function of satellite cells [84]. However, it remains to be studied whether satellite cells also contribute to muscle fiber metabolic alterations. We hypothesize that there is a strong interrelationship between skeletal muscle mass and metabolic regulation during recovery from atrophy, and that satellite cells exert a central role in their coordination.

\section{Technical implications}

Muscle quantitative and qualitative alterations in COPD patients have been extensively researched, albeit in isolation. However, considering the clear overlap in processes, and the recent indications for interrelationships between skeletal muscle mass and metabolic plasticity, there is a need to study these regulatory processes in an integrative manner (Figure 3). Integrative assessment of processes should occur at several levels. First, as the regulation of processes is studied by the assessment of static mRNA and protein markers, a panel of markers should be composed to assess the activation status of a process. Secondly, both anabolic and catabolic processes should be studied simultaneously to indicate the net turnover regulation. In addition, the regulation of protein and myonuclear turnover and, ideally, even the regulation of muscle mass and metabolism should be studied together.

As the processes regulating skeletal muscle remodeling are transiently activated upon external triggers, and are assessed by static markers, the timing of measurements is essential for the identification of relevant process activation, as well as for the subsequent interpretation. The dependence on static markers has limited the knowledge on the temporal regulation of remodeling-related processes. Although serial measurements after initiation of skeletal muscle remodeling would provide more insights in this temporal response, this is not feasible in humans. Therefore, rodent models are used to provide a better time-resolution of skeletal muscle remodeling/ recovery-related processes. Still, animal studies provide limited flexibility to study the causal relationship between protein-, mitochondrial-, and myonuclear turnoverrelated processes, as these processes are dynamic and seem to be highly interrelated. Recent advances have been made to perform in vivo measurements of protein turnover 
A

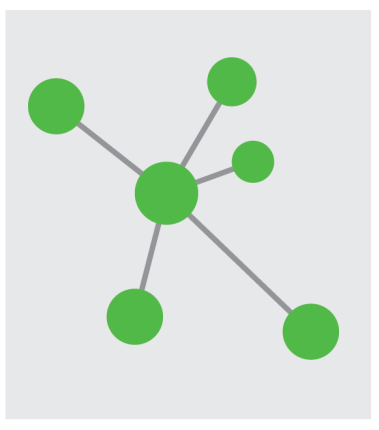

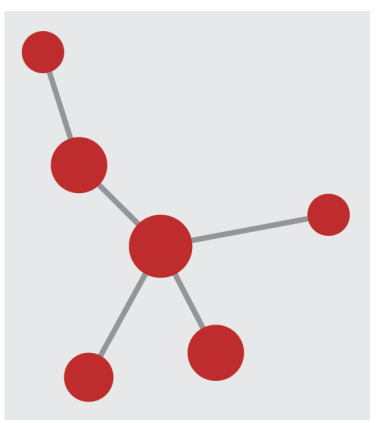

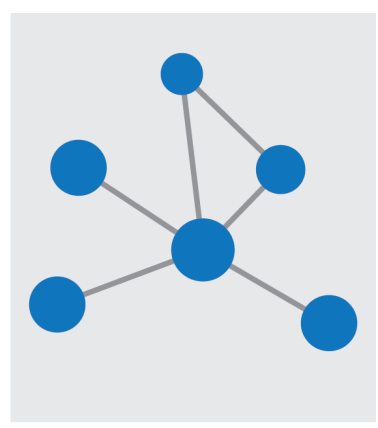

B
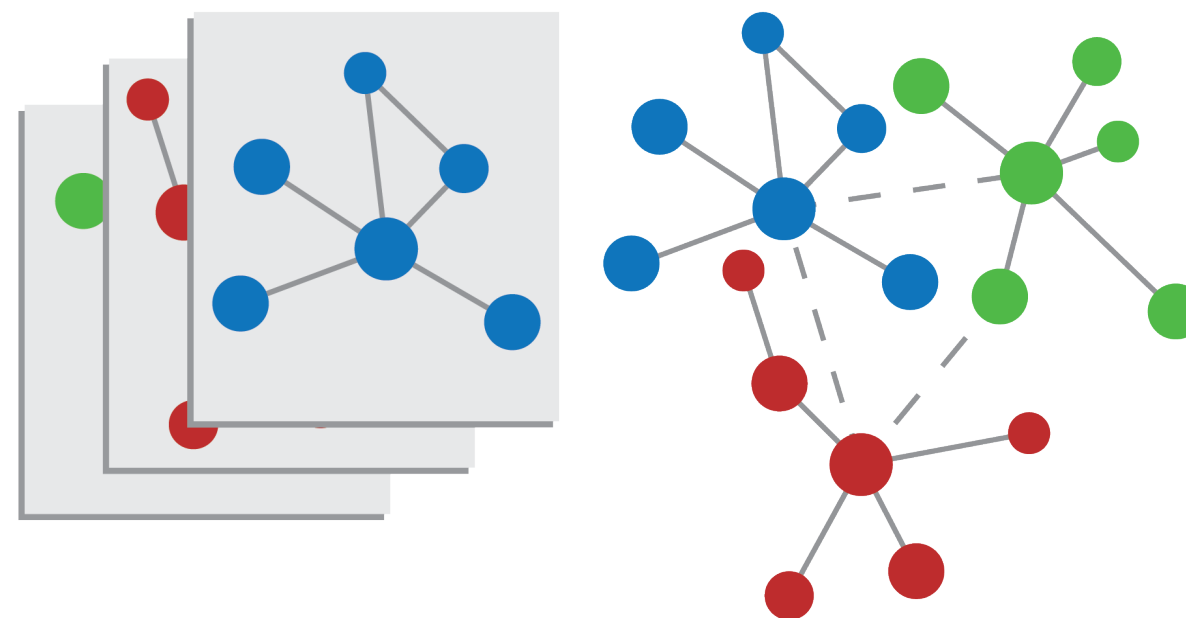

Figure 3. | Integrative assessment of muscle mass and metabolic plasticity. Schematic presentation of the integrative assessment of muscle mass and metabolic plasticity regulation. A. Integration of a panel of markers indicating molecular regulation of protein synthesis (green), protein degradation (red), and myogenesis (blue). Classically studied as separate processes. B) Combined assessment of anabolic and catabolic signaling, studied using an integrative approach to assess the interrelationship between protein synthesis, protein degradation, and myogenesis signaling, and their coordination.

and mitochondrial turnover [85-88]. Moreover, the regulatory pathways are widely studied, and largely known. In contrast, in vivo assessment of myonuclear turnover remains complicated, e.g. due to the difficulty to efficiently label and trace myonuclei, and the low basal turnover rate. Hence, direct assessments of myonuclear turnover have not yet been performed in humans. Moreover, there is a lack of knowledge on specific molecular checkpoints and processes regulating myonuclear turnover, while important signaling mechanisms involved in the activation of proliferation and differentiation are well-established. Until recently, the molecular regulation of satellite cell fusion was under-explored. A first opportunity to assess the regulation of satellite cell fusion in vivo was presented by the recent identification of satellite cell fusion mediators [89-91]. Yet, the development of knowledge on the regulation and role of 


\section{CHAPTER 1}

satellite cell fusion in both muscle mass and metabolic plasticity would benefit from a more efficient in vitro assessment tool.

\section{A potential role for impaired skeletal muscle recovery?}

As described, there are clear indications for alterations in several mechanisms regulating skeletal muscle mass and metabolism in patients with COPD. However, the current methodological and technical limitations hamper addressing their direct implications for skeletal muscle recovery. In this thesis, we therefore set out the development and use of novel strategies for the assessment of molecular disturbances in the regulation of skeletal muscle mass and quality in COPD patients, and discuss the implications for the development of COPD-related muscle pathology.

\section{OUTLINE THESIS}

The overall objective of the work described in this thesis is to study the mechanisms involved in skeletal muscle recovery, and to assess the implications of potential disturbances in these processes for skeletal muscle maintenance in COPD patients.

In Chapter 2, we review recent insights on the diagnosis and pathophysiology of muscle wasting in COPD, to establish the current knowledge on the molecular alterations underlying muscle atrophy in COPD. Furthermore, we describe the potential implications of these insights for established and novel therapies, and recommend directions for future research.

In Chapter 3, we perform a comprehensive assessment and comparison of the basal molecular regulation of protein degradation, protein synthesis, and myogenesis in skeletal muscles tissue of muscle-wasted COPD patients, non-wasted COPD patients, and healthy controls, to identify potential differentially regulated processes in a basal state.

The molecular regulation of muscle quality and quantity is important for the response to rehabilitation, which is highly variable between COPD patients. Next, we therefore aim to identify potential differences in the skeletal muscle regenerative response between COPD patients. To this end, we first perform an integrative assessment of the molecular processes implicated in the regulation of muscle quality and quantity during recovery following disuse in healthy humans and rodents, in Chapter $\mathbf{4}$. Secondly, we use an unbiased, clustering based analysis, to identify groups of COPD patients with a differential molecular rehabilitation response, and extensively characterize these 
patient groups for physiological and functional differences in Chapter $\mathbf{5}$.

To elucidate the role and regulation of postnatal myonuclear accretion in skeletal muscle mass and metabolic plasticity, we aim to study the functional contribution of satellite cells to muscle fiber recovery after atrophy or damage. To this end, we have developed an in vitro model that better reflects in vivo postnatal myogenesis. The model, and its use to study cell intrinsic and extrinsic processes and signaling involved in the regulation of postnatal myogenesis, are discussed in Chapter 6. In Chapter 7, we use this in vitro approach to study the triggers and physiological role of myonuclear accretion in the regulation of muscle fiber mass and metabolic plasticity.

Finally, the implications of the experimental findings for the regulation of skeletal muscle atrophy-recovery in health and disease, and for the development of COPDinduced skeletal muscle pathology are discussed in Chapter $\mathbf{8}$. 


\section{CHAPTER 1}

\section{REFERENCES}

1. Egan B, Zierath JR. Exercise metabolism and the molecular regulation of skeletal muscle adaptation. Cell Metab 2013;17(2):162-84.

2. McGlory C, Phillips SM. Exercise and the Regulation of Skeletal Muscle Hypertrophy. Prog Mol Biol Transl Sci 2015;135:153-73.

3. Brooks NE, Myburgh KH. Skeletal muscle wasting with disuse atrophy is multi-dimensional: the response and interaction of myonuclei, satellite cells and signaling pathways. Frontiers in physiology 2014;5:99.

4. Cohen S, Nathan JA, Goldberg AL. Muscle wasting in disease: molecular mechanisms and promising therapies. Nat Rev Drug Discov 2015;14(1):58-74.[published Online First: 2015/01/01]

5. Schols AM, Broekhuizen R, Weling-Scheepers CA, et al. Body composition and mortality in chronic obstructive pulmonary disease. Am J Clin Nutr 2005;82(1):53-9.

6. Metter EJ, Talbot LA, Schrager M, et al. Skeletal muscle strength as a predictor of all-cause mortality in healthy men. J Gerontol A Biol Sci Med Sci 2002;57(10):B359-65.[published Online First: 2002/09/21]

7. Wolfe RR. The underappreciated role of muscle in health and disease. Am J Clin Nutr 2006;84(3):475-82.

8. Trajanoska K, Schoufour JD, Darweesh SK, et al. Sarcopenia and Its Clinical Correlates in the General Population: The Rotterdam Study. J Bone Miner Res 2018

9. Bilodeau PA, Coyne ES, Wing SS. The ubiquitin proteasome system in atrophying skeletal muscle: roles and regulation. Am J Physiol Cell Physiol 2016;311(3):C392-403.

10. Langen RC, Gosker HR, Remels AH, et al. Triggers and mechanisms of skeletal muscle wasting in chronic obstructive pulmonary disease. Int J Biochem Cell Biol 2013;45(10):224556.

11. Frost RA, Lang $\mathrm{CH}$. Protein kinase B/Akt: a nexus of growth factor and cytokine signaling in determining muscle mass. Journal of applied physiology 2007;103(1):378-87.

12. Egerman MA, Glass DJ. Signaling pathways controlling skeletal muscle mass. Crit Rev Biochem Mol Biol 2014;49(1):59-68.

13. Saxton RA, Sabatini DM. mTOR Signaling in Growth, Metabolism, and Disease. Cell 2017;168(6):960-76.

14. Martin-Rincon M, Morales-Alamo D, Calbet JAL. Exercise-mediated modulation of autophagy in skeletal muscle. Scand J Med Sci Sports 2018;28(3):772-81.

15. Fujita $\mathrm{S}$, Dreyer $\mathrm{HC}$, Drummond $\mathrm{MJ}$, et al. Nutrient signalling in the regulation of human muscle protein synthesis. J Physiol 2007;582(Pt 2):813-23.

16. Witczak CA, Sharoff CG, Goodyear LJ. AMP-activated protein kinase in skeletal muscle: from structure and localization to its role as a master regulator of cellular metabolism. Cell Mol Life Sci 2008;65(23):3737-55.

17. Rudrappa SS, Wilkinson DJ, Greenhaff PL, et al. Human Skeletal Muscle Disuse Atrophy: Effects on Muscle Protein Synthesis, Breakdown, and Insulin Resistance-A Qualitative Review. Frontiers in physiology 2016;7:361.

18. Schiaffino $S$, Dyar KA, Ciciliot $S$, et al. Mechanisms regulating skeletal muscle growth and atrophy. FEBS J 2013;280(17):4294-314.

19. Wall BT, Dirks ML, van Loon LJ. Skeletal muscle atrophy during short-term disuse: implications for age-related sarcopenia. Ageing Res Rev 2013;12(4):898-906.

20. Laudisio A, Costanzo L, Di Gioia C, et al. Dietary intake of elderly outpatients with chronic obstructive pulmonary disease. Arch Gerontol Geriatr 2016;64:75-81. 
21. Tieland $\mathrm{M}$, Borgonjen-Van den Berg KJ, Van Loon LJ, et al. Dietary Protein Intake in Dutch Elderly People: A Focus on Protein Sources. Nutrients 2015;7(12):9697-706.

22. Bell KE, von Allmen MT, Devries MC, et al. Muscle Disuse as a Pivotal Problem in Sarcopeniarelated Muscle Loss and Dysfunction. J Frailty Aging 2016;5(1):33-41.

23. Pitta F, Troosters T, Spruit MA, et al. Characteristics of physical activities in daily life in chronic obstructive pulmonary disease. Am J Respir Crit Care Med 2005;171(9):972-7.

24. Wall BT, Dirks ML, Snijders T, et al. Short-term muscle disuse lowers myofibrillar protein synthesis rates and induces anabolic resistance to protein ingestion. Am J Physiol Endocrinol Metab 2016;310(2):E137-47.

25. Glover El, Phillips SM, Oates BR, et al. Immobilization induces anabolic resistance in human myofibrillar protein synthesis with low and high dose amino acid infusion. J Physiol 2008;586(24):6049-61.

26. Dirks AJ, Leeuwenburgh C. Tumor necrosis factor alpha signaling in skeletal muscle: effects of age and caloric restriction. J Nutr Biochem 2006;17(8):501-8.

27. Fanzani A, Conraads VM, Penna F, et al. Molecular and cellular mechanisms of skeletal muscle atrophy: an update. J Cachexia Sarcopenia Muscle 2012;3(3):163-79.

28. Ceelen JJM, Schols A, van Hoof SJ, et al. Differential regulation of muscle protein turnover in response to emphysema and acute pulmonary inflammation. Respir Res 2017;18(1):75.

29. de Theije C, Costes F, Langen RC, et al. Hypoxia and muscle maintenance regulation: implications for chronic respiratory disease. Current opinion in clinical nutrition and metabolic care 2011;14(6):548-53.

30. Ceelen JJM, Schols A, Kneppers AEM, et al. Altered protein turnover signaling and myogenesis during impaired recovery of inflammation-induced muscle atrophy in emphysematous mice. Sci Rep 2018;8(1):10761.[published Online First: 2018/07/19]

31. Dideriksen K, Boesen AP, Kristiansen JF, et al. Skeletal muscle adaptation to immobilization and subsequent retraining in elderly men: No effect of anti-inflammatory medication. Exp Gerontol 2016;82:8-18.

32. Tanner RE, Brunker LB, Agergaard J, et al. Age-related differences in lean mass, protein synthesis and skeletal muscle markers of proteolysis after bed rest and exercise rehabilitation. J Physiol 2015;593(18):4259-73.

33. Pansters NA, Schols AM, Verhees KJ, et al. Muscle-specific GSK-3beta ablation accelerates regeneration of disuse-atrophied skeletal muscle. Biochim Biophys Acta 2015;1852(3):490506.

34. Baehr LM, West DW, Marcotte G, et al. Age-related deficits in skeletal muscle recovery following disuse are associated with neuromuscular junction instability and ER stress, not impaired protein synthesis. Aging (Albany NY) 2016;8(1):127-46.

35. Mitchell CJ, D'Souza RF, Mitchell SM, et al. Impact of dairy protein during limb immobilization and recovery on muscle size and protein synthesis; a randomized controlled trial. Journal of applied physiology 2018;124(3):717-28.

36. Jones SW, Hill RJ, Krasney PA, et al. Disuse atrophy and exercise rehabilitation in humans profoundly affects the expression of genes associated with the regulation of skeletal muscle mass. FASEB journal : official publication of the Federation of American Societies for Experimental Biology 2004;18(9):1025-7.

37. Suetta C, Frandsen U, Mackey AL, et al. Ageing is associated with diminished muscle regrowth and myogenic precursor cell expansion early after immobility-induced atrophy in human skeletal muscle. J Physiol 2013;591(15):3789-804.

38. Snijders T, Nederveen JP, McKay BR, et al. Satellite cells in human skeletal muscle plasticity. Frontiers in physiology 2015;6:283.

39. Relaix F, Zammit PS. Satellite cells are essential for skeletal muscle regeneration: the cell on the edge returns centre stage. Development 2012;139(16):2845-56. 


\section{CHAPTER 1}

40. Davis TA, Fiorotto ML. Regulation of muscle growth in neonates. Current opinion in clinical nutrition and metabolic care 2009;12(1):78-85.

41. Wang YX, Rudnicki MA. Satellite cells, the engines of muscle repair. Nat Rev Mol Cell Biol 2011;13(2):127-33.

42. Dhawan J, Rando TA. Stem cells in postnatal myogenesis: molecular mechanisms of satellite cell quiescence, activation and replenishment. Trends in cell biology 2005;15(12):666-73.

43. Yin $H$, Price F, Rudnicki MA. Satellite cells and the muscle stem cell niche. Physiol Rev 2013;93(1):23-67.

44. Rosenblatt JD, Parry DJ. Gamma irradiation prevents compensatory hypertrophy of overloaded mouse extensor digitorum longus muscle. Journal of applied physiology 1992;73(6):2538-43.

45. Rosenblatt JD, Parry DJ. Adaptation of rat extensor digitorum longus muscle to gamma irradiation and overload. Pflugers Archiv : European journal of physiology 1993;423(34):255-64.

46. Fry CS, Lee JD, Jackson JR, et al. Regulation of the muscle fiber microenvironment by activated satellite cells during hypertrophy. FASEB journal : official publication of the Federation of American Societies for Experimental Biology 2014;28(4):1654-65.

47. Egner IM, Bruusgaard JC, Gundersen K. Satellite cell depletion prevents fiber hypertrophy in skeletal muscle. Development 2016;143(16):2898-906.

48. Goh Q, Millay DP. Requirement of myomaker-mediated stem cell fusion for skeletal muscle hypertrophy. Elife 2017;6

49. Suetta C, Hvid LG, Justesen L, et al. Effects of aging on human skeletal muscle after immobilization and retraining. Journal of applied physiology 2009;107(4):1172-80.

50. Joanisse S, Nederveen JP, Snijders $T$, et al. Skeletal Muscle Regeneration, Repair and Remodelling in Aging: The Importance of Muscle Stem Cells and Vascularization. Gerontology 2017;63(1):91-100.

51. Theriault ME, Pare ME, Lemire BB, et al. Regenerative defect in vastus lateralis muscle of patients with chronic obstructive pulmonary disease. Respir Res 2014;15:35.

52. Kumar V, Selby A, Rankin D, et al. Age-related differences in the dose-response relationship of muscle protein synthesis to resistance exercise in young and old men. J Physiol 2009;587(1):211-7.

53. Fry CS, Drummond MJ, Glynn EL, et al. Aging impairs contraction-induced human skeletal muscle mTORC1 signaling and protein synthesis. Skelet Muscle 2011;1(1):11.

54. De Brandt J, Spruit MA, Derave W, et al. Changes in structural and metabolic muscle characteristics following exercise-based interventions in patients with COPD: a systematic review. Expert Rev Respir Med 2016;10(5):521-45.

55. Morton RW, McGlory C, Phillips SM. Nutritional interventions to augment resistance training-induced skeletal muscle hypertrophy. Frontiers in physiology 2015;6:245.

56. van de Bool C, Rutten EPA, van Helvoort $A$, et al. A randomized clinical trial investigating the efficacy of targeted nutrition as adjunct to exercise training in COPD. J Cachexia Sarcopenia Muscle 2017;8(5):748-58.

57. Camillo CA, Osadnik CR, van Remoortel $\mathrm{H}$, et al. Effect of "add-on" interventions on exercise training in individuals with COPD: a systematic review. ERJ Open Res 2016;2(1)

58. Creutzberg EC, Wouters EF, Mostert R, et al. A role for anabolic steroids in the rehabilitation of patients with COPD? A double-blind, placebo-controlled, randomized trial. Chest 2003;124(5):1733-42.

59. Snijders T, Verdijk LB, van Loon LJ. The impact of sarcopenia and exercise training on skeletal muscle satellite cells. Ageing Res Rev 2009;8(4):328-38.

60. Theriault ME, Pare ME, Maltais F, et al. Satellite cells senescence in limb muscle of severe 
patients with COPD. PLoS One 2012;7(6):e39124.

61. Dreyer HC, Blanco CE, Sattler FR, et al. Satellite cell numbers in young and older men 24 hours after eccentric exercise. Muscle Nerve 2006;33(2):242-53.

62. Snijders T, Verdijk LB, Smeets JS, et al. The skeletal muscle satellite cell response to a single bout of resistance-type exercise is delayed with aging in men. Age (Dordr) 2014;36(4):9699.

63. Pomies $\mathrm{P}$, Rodriguez J, Blaquiere $\mathrm{M}$, et al. Reduced myotube diameter, atrophic signalling and elevated oxidative stress in cultured satellite cells from COPD patients. J Cell Mol Med 2015;19(1):175-86.

64. Maltais F, Decramer M, Casaburi R, et al. An official American Thoracic Society/European Respiratory Society statement: update on limb muscle dysfunction in chronic obstructive pulmonary disease. Am J Respir Crit Care Med 2014;189(9):e15-62.

65. Distefano G, Goodpaster BH. Effects of Exercise and Aging on Skeletal Muscle. Cold Spring Harb Perspect Med 2018;8(3)

66. McGregor RA, Cameron-Smith D, Poppitt SD. It is not just muscle mass: a review of muscle quality, composition and metabolism during ageing as determinants of muscle function and mobility in later life. Longev Healthspan 2014;3(1):9.

67. Robles PG, Sussman MS, Naraghi A, et al. Intramuscular Fat Infiltration Contributes to Impaired Muscle Function in COPD. Medicine and science in sports and exercise 2015;47(7):1334-41.

68. Latroche C, Gitiaux C, Chretien F, et al. Skeletal Muscle Microvasculature: A Highly Dynamic Lifeline. Physiology (Bethesda) 2015;30(6):417-27.

69. Landers-Ramos RQ, Prior SJ. The Microvasculature and Skeletal Muscle Health in Aging. Exerc Sport Sci Rev 2018

70. Liu W, Klose A, Forman S, et al. Loss of adult skeletal muscle stem cells drives age-related neuromuscular junction degeneration. Elife 2017;6

71. Gonzalez-Freire M, de Cabo R, Studenski SA, et al. The Neuromuscular Junction: Aging at the Crossroad between Nerves and Muscle. Front Aging Neurosci 2014;6:208.

72. Romanello V, Sandri M. Mitochondrial Quality Control and Muscle Mass Maintenance. Frontiers in physiology 2015;6:422.

73. Fragala MS, Kenny AM, Kuchel GA. Muscle quality in aging: a multi-dimensional approach to muscle functioning with applications for treatment. Sports Med 2015;45(5):641-58.

74. MacMillan NJ, Kapchinsky S, Konokhova Y, et al. Eccentric Ergometer Training Promotes Locomotor Muscle Strength but Not Mitochondrial Adaptation in Patients with Severe Chronic Obstructive Pulmonary Disease. Frontiers in physiology 2017;8:114.

75. Call JA, Wilson RJ, Laker RC, et al. Ulk1-mediated autophagy plays an essential role in mitochondrial remodeling and functional regeneration of skeletal muscle. Am J Physiol Cell Physiol 2017;312(6):C724-C32.

76. Vainshtein A, Grumati P, Sandri M, et al. Skeletal muscle, autophagy, and physical activity: the menage a trois of metabolic regulation in health and disease. J Mol Med (Berl) 2014;92(2):127-37.

77. Sanchez AM, Bernardi H, Py G, et al. Autophagy is essential to support skeletal muscle plasticity in response to endurance exercise. Am J Physiol Regul Integr Comp Physiol 2014;307(8):R956-69.

78. Tam BT, Pei XM, Yu AP, et al. Autophagic adaptation is associated with exercise-induced fibre-type shifting in skeletal muscle. Acta Physiol (Oxf) 2015;214(2):221-36.

79. Fritzen $A M$, Madsen $A B$, Kleinert $M$, et al. Regulation of autophagy in human skeletal muscle: effects of exercise, exercise training and insulin stimulation. J Physiol 2016;594(3):745-61.

80. Drummond MJ, Fry CS, Glynn EL, et al. Rapamycin administration in humans blocks the contraction-induced increase in skeletal muscle protein synthesis. J Physiol 2009;587(Pt 


\section{CHAPTER 1}

7):1535-46.

81. Morita M, Gravel SP, Hulea $L$, et al. mTOR coordinates protein synthesis, mitochondrial activity and proliferation. Cell Cycle 2015;14(4):473-80.

82. Abreu P, Mendes SV, Ceccatto VM, et al. Satellite cell activation induced by aerobic muscle adaptation in response to endurance exercise in humans and rodents. Life Sci 2017;170:3340.

83. Fry CS, Lee JD, Mula J, et al. Inducible depletion of satellite cells in adult, sedentary mice impairs muscle regenerative capacity without affecting sarcopenia. Nat Med 2015;21(1):7680.

84. Burd NA, De Lisio M. Skeletal Muscle Remodeling: Interconnections Between Stem Cells and Protein Turnover. Exerc Sport Sci Rev 2017;45(3):187-91.

85. Chan XC, Black CM, Lin AJ, et al. Mitochondrial protein turnover: methods to measure turnover rates on a large scale. J Mol Cell Cardiol 2015;78:54-61.

86. Williams JA, Zhao K, Jin S, et al. New methods for monitoring mitochondrial biogenesis and mitophagy in vitro and in vivo. Exp Biol Med (Maywood) 2017;242(8):781-87.

87. Hellerstein $M$, Evans W. Recent advances for measurement of protein synthesis rates, use of the 'Virtual Biopsy' approach, and measurement of muscle mass. Current opinion in clinical nutrition and metabolic care 2017;20(3):191-200.

88. Wilkinson DJ, Brook MS, Smith K, et al. Stable isotope tracers and exercise physiology: past, present and future. J Physiol 2017;595(9):2873-82.

89. Millay DP, O'Rourke JR, Sutherland LB, et al. Myomaker is a membrane activator of myoblast fusion and muscle formation. Nature 2013;499(7458):301-5.

90. Bi P, McAnally JR, Shelton JM, et al. Fusogenic micropeptide Myomixer is essential for satellite cell fusion and muscle regeneration. Proceedings of the National Academy of Sciences 2018;115(15):3864-69.

91. Kim GW, Nam GH, Kim IS, et al. Xk-related protein 8 regulates myoblast differentiation and survival. FEBS J 2017;284(21):3575-88. 
CACHEXIA IN CHRONIC OBSTRUKTIVE PULMONARY DISEASE: NEW INSIGHTS AND THERAPEUTIC PERSPECTIVE

J Cachexia Sarcopenia Muscle 2016;7(1):5-22.

KARIN J.C. SANDERS* ANITA E.M. KNERPERS* COBY VAN DE BQOL RAMON C.J. LANGEN \& ANNEMIE M.W.J. SCHOLS *these authors contributed equally 


\section{ABSTRACT}

Cachexia and muscle wasting are well recognized as common and partly reversible features of chronic obstructive pulmonary disease (COPD) adversely affecting disease progression and prognosis. This argues for integration of weight and muscle maintenance in patient care. In this review, recent insights are presented in the diagnosis of muscle wasting in COPD, the pathophysiology of muscle wasting, and putative mechanisms involved in a disturbed energy balance as cachexia driver. We discuss the therapeutic implications of these new insights for optimizing and personalizing management of COPD-induced cachexia. 


\section{INTRODUCTION}

Chronic obstructive pulmonary disease (COPD) is one of the leading causes of death worldwide. It has been estimated that COPD-related mortality rates will even increase in the coming decades. This increase is not only related to the prevalence of smoking, but also ageing and reduced mortality from other common causes of death play a role [1]. Additionally, COPD is a major contributor to global disease burden, accounting for 43.3 million disability-adjusted life years in 2010 [2]. The disease is characterized by persistent airflow obstruction, resulting from inflammation and remodeling of the airways, and may include development of emphysema. Furthermore, systemic disease manifestations and acute exacerbations influence disease burden and mortality risk [3]. Extending the classical descriptions of the 'pink puffer' and 'blue bloater', recent unbiased statistical approaches [4,5] support the concept that body weight and body composition discriminate pulmonary phenotypes and are predictors of outcome. Extra-pulmonary degenerative manifestations that may occur in COPD include osteoporosis [6] and muscle wasting. The prevalence of muscle wasting is relatively high in COPD: 15-40\% depending on definition and disease stage [7, 8]. Importantly, muscle wasting not only contributes to diminished skeletal muscle function, reduced exercise capacity, and decreased health status $[9,10]$, but is also a determinant of mortality in COPD, independent of airflow obstruction $[8,11]$.

Muscle wasting in COPD has been demonstrated by decreases in fat-free mass (FFM) at whole body level, but also specifically at the level of the extremities [12]. Whole body and trunk FFM reduction are more pronounced in the emphysematous phenotype, whereas reduced FFM in extremities is not different between the pulmonary phenotypes $[13,14]$. In addition, muscle wasting is apparent as a decrease in the size of individual muscle fibers, and this muscle fiber atrophy in COPD seems selective for type II fibers in peripheral muscle $[15,16]$, which is in line with other chronic diseases prone to cachexia such as chronic heart failure [17]. Furthermore, a shift in muscle fiber composition from type I (oxidative) to type II (glycolytic), accompanied by a decrease in oxidative capacity, culminates in reduced muscle endurance [18]. This not only contributes to reduced exercise capacity [19] but may also affect muscle mass in COPD [20], because type I and II fibers display different responses to anabolic and catabolic signals $[21,22]$.

While unintended weight loss was initially considered to be an indicator of inevitable and terminal progression of the disease process, there is now convincing evidence that it is an independent determinant of survival, arguing for weight maintenance in 


\section{CHAPTER 2}

patient care. There are indications that the pathophysiology of unintended weight loss is different between clinically stable COPD and during acute flare-ups of the disease. To date, data in acute exacerbations of COPD are, however, very limited. Therefore, lung cancer is used as a comparative acute pulmonary cachexia model.

A recent unbiased statistical approach suggests that not all COPD patients but only the emphysematous phenotype is prone to cachexia [4], although the informative value of available clinical studies is limited by a cross-sectional study design. The last two decades have also yielded insight in the impairments of the processes governing muscle mass and identified putative triggers of muscle wasting in COPD. However, it remains unclear to what extent acute flare-ups of COPD may accelerate chronic wasting of muscle mass and whether muscle wasting involves similar mechanisms as in other chronic diseases or in lung cancer cachexia. In this review, we present recent insights in the pathophysiology of muscle wasting in COPD and (putative) mechanisms involved in the pathophysiology of a disturbed energy balance as important driver of cachexia, which may lead to novel targets for clinical management of cachexia in COPD.

\section{Recent developments in identifying muscle wasting in COPD}

Incorporation of body composition into nutritional assessment has been a major step forward in understanding systemic COPD pathophysiology, since changes in weight and classification of body mass index (BMI) do not account for (hidden) body compositional shifts in fat mass (FM), FFM, and bone mineral density. In clinical research, bioelectrical impedance analysis is commonly used to identify cachexia. Traditionally, reference values for fat-free mass index (FFMI) in COPD were developed based on age-specific and gender-specific 10th percentile values [8]. These reference values were defined as abnormally low, based on well-established adverse effects of low FFMI on physical performance and survival in normal to underweight COPD patients [7, 11]. However, this might underestimate low muscle mass in the increasing proportion of overweight to obese COPD patients [23]. The recent European Respiratory Society statement on nutritional assessment and therapy in COPD [24] proposed dual-energy X-ray absorptiometry (DEXA) as the most appropriate method for body composition analysis in COPD, mostly because it combines screening for osteoporosis with assessment of FM and FFM at the regional level in addition to whole body level. Consequently, body composition assessed by DEXA also allows measurement of appendicular skeletal muscle mass (ASM), which has been demonstrated to be stronger related to physical functioning than total FFM [23]. Moreover, we recently showed that this particularly applies to overweight to obese COPD patients [23, 25]. 
Whereas low muscle mass is prevalent in $\pm 15 \%$ of well-functioning elderly in the general aged population [26], a higher prevalence can be expected in COPD as a reflection of accelerated ageing [25]. Indeed, Van de Bool et al. recently identified low ASM in even $87 \%$ of Dutch COPD patients eligible for pulmonary rehabilitation, along with a high persisting prevalence across all BMI categories [23]. The ASM-wasted phenotype was not only associated with impaired strength but in men also with decreased endurance capacity. Coexistent abdominal obesity was identified in 78\% of muscle-wasted patients, which appeared to have a protective effect on physical functioning [23]. Physiological alterations in terms of less hyperinflation and a larger inspiratory capacity in obese COPD patients contribute to certain advantages during physical activity [27]. In addition, mortality rates in advanced COPD are the lowest among obese subjects [28]. This prognostic advantage typically reflects the 'obesity paradox', because obesity, on the other hand, is also associated with increased risk of cardiovascular and metabolic diseases.

Although clinically useful estimates can be derived by DEXA, a more precise distinction between muscle mass, and visceral and subcutaneous adipose tissue, requires more advanced imaging technologies. This could be relevant in COPD as Van den Borst et al. and Furutate et al. recently reported a higher visceral adipose tissue in older-age patients with COPD compared with age-matched healthy controls, despite comparable subcutaneous adipose tissue and BMI $[29,30]$.

McDonald et al. [31] recently demonstrated that CT-derived pectoralis muscle area (PMA) provides a more clinically relevant measure of COPD-related outcomes in comparison with BMI, as lower PMA was associated with more severe expiratory airflow obstruction, lower quality of life, and impaired exercise capacity. Because gender differences have been documented in body composition and its functional implications [23], Diaz et al. explored gender differences in computed tomography (CT)-derived PMA and observed lower PMA in women compared with men [32]. CT scans are often used to exclude other underlying illnesses, and therefore, chest CTderived analysis of body composition may be an attractive diagnostic tool to combine screening for pulmonary and systemic pathology. However, it first needs to be properly validated against reference methods of whole body and regional body composition to allow use in clinical practice. Furthermore, due to the radiation exposure, use of CT scans for body composition assessment is only admissible when scans are already performed to screen for pulmonary pathology. 


\section{CHAPTER 2}

\section{New insights in the pathophysiology of muscle wasting in chronic obstructive pulmonary disease}

The loss of muscle mass and cross-sectional area in COPD patients as determined by imaging techniques has been confirmed at the cellular level, that is, a reduction in muscle fiber cross-sectional area [16]. As reviewed by Langen et al. [33] and Remels et al. [20], triggers of muscle wasting include hypoxemia, oxidative stress, inflammation, impaired growth factor signaling, oral glucocorticoids, disuse, and malnutrition, some of which are influenced by smoking [34]. Wasting of skeletal muscle is due to a net catabolic state, which may result from an imbalance in muscle protein synthesis and breakdown (i.e., protein turnover), as well as from an imbalance in myonuclear accretion and loss (i.e., myonuclear turnover).

\section{Protein turnover}

To get insight in the rate of (muscle) protein turnover in COPD, information on (muscle) protein synthesis and breakdown is required. Both increased and normal rates of whole body protein turnover have been reported in patients with COPD [35, 36], but the relative contribution of muscle versus other tissues to protein turnover is unknown. Rutten et al. observed an increase in myofibrillar protein breakdown in cachectic COPD patients compared with non-cachectic patients and controls [35], but no data are available regarding muscle protein synthesis rate, except for a small study showing depressed muscle protein synthesis rates in malnourished patients with emphysema [37]. Numerous studies, however, have addressed molecular regulation of anabolic and catabolic pathways in the quadriceps muscle of COPD patients, which provides some insight in altered muscle protein turnover in muscle-wasted COPD patients.

\section{Proteolytic signaling}

Several environmental triggers can lead to catabolic signaling in the skeletal muscle, mediated by transcriptional regulators including nuclear factor kappa-light-chainenhancer of activated $\mathrm{B}$ cells (NF-KB) and forkhead box $\mathrm{O}$ transcription factors (FOXO). NF-KB activity is increased in COPD patients compared with controls [38, 39] and in cachectic COPD patients compared with non-cachectic COPD patients [38, 40]. Furthermore, limb muscle NF-KB activity is increased in patients with lung cancer cachexia [41]. FOXO mRNA and protein expression are increased in patients with COPD $[38,39,42-45]$, seemingly independent of body composition, although it is noticeable that in all studies, the patient group showed signs of emphysema. In the COPD patient group, FOXO-1 protein expression was higher in limb muscles than in respiratory muscles, while this difference was not found in controls [46]. Interestingly, the 
respiratory muscles of COPD patients show an opposite fiber type shift compared with limb muscles, that is, towards more type I fibers $[47,48]$. This will have implications for the expression levels of constituents of atrophy signaling pathways [22, 49]. Increased catabolic signaling through FOXO and NF-KB can induce gene expression of key factors in both the ubiquitin proteasome system (UPS) $[50,51]$ and the autophagy lysosome pathway [33, 52].

\section{Ubiquitin proteasome-mediated degradation}

The ubiquitin 26S-proteasome pathway consists of coordinated actions of the ubiquitin conjugating and ligating enzymes that link ubiquitin chains onto proteins to mark them for degradation by the proteasome [53,54]. These enzymes include tripartite motif containing 63, E3 ubiquitin protein ligase (TRIM63, referred to as MURF1), F-box protein 32 (FBXO32, referred to as ATROGIN1), and neural precursor cell expressed, developmentally downregulated 4, E3 ubiquitin protein (NEDD4).

MURF1 limb and respiratory muscle mRNA and protein expression appear unaltered in COPD patients compared with controls [38, 40,55-57], although one study reported increased MURF1 protein expression in the limb muscles of cachectic COPD patients [39]. In COPD patients, MURF1 protein expression is relatively increased in limb muscle than in the respiratory muscle, while this difference was not found in controls [46]. Furthermore, cachectic COPD patients show increased limb muscle mRNA expression of MURF1 compared with a control population [44]. ATROGIN1 mRNA and protein expression are increased in limb muscles [38-40, 44, 56, 57], but unaltered in respiratory muscles of patients with COPD [55]. Similarly, ATROGIN1 mRNA expression is increased in the limb muscles of smokers [58]. Furthermore, Doucet et al. found that COPD patients display a higher ATROGIN1 protein expression in limb muscles than in respiratory muscles, while this difference was not found in controls [46]. Additionally, limb muscle NEDD4 protein expression is increased in patients with COPD [57]. Total poly-ubiquitinated protein is increased in limb muscles of COPD patients compared with healthy controls $[39,56]$, and in cachectic COPD patients compared with noncachectic COPD patients [38].

Taken together, the majority of the literature suggests that wasting in COPD is accompanied by an increase in UPS activation. The increase in catabolic signaling in cachectic COPD patients is site-specific. This may reflect disuse atrophy of the limb muscle with maintained or increased respiratory muscle activity, or it may result from an interaction between inactivity and other triggers of atrophy, such as smoking. 


\section{CHAPTER 2}

\section{Autophagy-lysosome-mediated degradation}

The autophagy-lysosome pathway is a protein degradation pathway, which recently gained interest in the context of COPD-associated muscle dysfunction. Upon activation, autophagosomes form and mature to subsequently fuse with lysosomes. The autophago-lysosomes degrade the cargo and release amino-acids for de novo protein synthesis or other metabolic fates [59].

Signaling through v-akt murine thymoma viral oncogene (AKT) regulates mechanistic target of rapamycin (serine/threonine kinase) complex 1 (MTORC1) activity and downstream of MTORC1, unc-51 like autophagy activating kinase 1 (ULK1) activity, thereby regulating autophagy initiation [60,61]. Inhibitory MTORC1-mediated ULK1 phosphorylation is decreased in limb muscles of COPD patients compared with controls [45], which may implicate an increase in autophagic flux induction. The increase of FOXO mRNA and protein expression in COPD patients may induce the transcription of autophagy-related genes. However, it should be taken into account that FOXO transcriptional activity is also regulated by post-translational modifications. Plant et al. found that the mRNA expression of autophagy-related genes Beclin-1 and microtubule-associated protein 1 light chain 3 alpha (MAP1LC3A, referred to as LC3) is unaltered in the limb muscles of COPD patients compared with controls [57]. Limb muscle sequestosome 1 (SQSTM1, referred to as P62) mRNA expression, however, is increased in COPD patients [45]. Although the mRNA expression of autophagy-related genes and the activation of ULK1 may give some insight in the level of autophagy initiation, it does not directly reflect the level of autophagic flux. In muscle biopsies, the conversion of LC3BI to LC3BII can be used as a measure for autophagic flux. Furthermore, P62 is used as a marker for autophagic flux, because it is broken down by the lysosome. In the limb muscles of COPD patients, the LC3BII/I ratio is increased $[39,45]$, pointing to an increase in the autophagic flux. In contrast, P62 protein expression is increased, pointing to a decrease in autophagic flux [45]. However, it cannot be excluded that the increase in P62 protein expression is due to the increase in P62 transcription. The number of autophagosomes was found to be increased in the limb muscle of COPD patients $[39,45]$, which suggests an increase in autophagic flux. However, it is only possible to speculate on the level of autophagic flux in the limb muscles of patients with COPD based on the currently applied markers, as these incompletely cover autophagic flux, autophagy induction, and autophagic-lysosomal degradation. Therefore, autophagic flux markers should be analyzed coupled to the activity status of upstream regulators such as MTORC1 and AMP-activated protein kinase (AMPK) in muscle biopsies. Moreover, besides its role in protein breakdown, autophagy also acts as a quality control mechanism for proteins and intracellular 
components $[62,63]$. Therefore, an impaired autophagic flux in COPD patients may have consequences for the integrity and function of intracellular components such as the nucleus and mitochondria.

It currently is unknown if the autophagic-lysosome pathway activity is altered during acute exacerbations of COPD, because most studies were conducted in stable COPD patients. However, in lung cancer cachexia, LC3BII protein expression and BCL2/ adenovirus E1B 19kDa interacting protein 3 (BNIP3) mRNA expression are induced [41], pointing to an increase in autophagy. From this, autophagy induction in skeletal muscle might be anticipated during acute stages of COPD wasting.

\section{Protein synthesis signaling}

A major anabolic pathway is the insulin-like growth factor 1 (IGF1)/phosphatidylinositol4,5-bisphosphate 3-kinase, catalytic subunit alpha (PIK3CA referred to as PI3K)/AKT pathway. Most studies found an increase in IGF1 mRNA expression in the limb muscle of COPD patients compared with controls [42, 43, 64], although Crul et al. found a decrease in IGF1 mRNA expression in stable COPD patients [65]. Unfortunately, this study did not provide body composition data. Cachectic COPD patients seem to have a lower limb muscle IGF1 mRNA and protein expression than non-cachectic COPD patients [40]. Furthermore, during an acute exacerbation, muscle IGF1 mRNA expression is lower in COPD patients than in controls, although IGF1 protein expression remains unaltered [65].

Even though IGF1 mRNA expression is increased in limb muscles of COPD patients, AKT activation remains unaltered $[39,45,57]$. AKT activity is relatively increased in cachectic patients compared with non-cachectic patients and healthy controls [40,44], while the decrease in IGF1 mRNA expression in this group would generally implicate a decrease of IGF1/AKT signaling. Interestingly, an increase in AKT activation is also observed in patients with lung cancer-related cachexia [41], suggesting it may be a common feature of pulmonary cachexia. The discrepancy in IGF1 mRNA expression and AKT activation suggests altered regulation at the IGF1 protein or IGF1 receptor level [66].

Signaling through AKT inhibits the upstream inhibitor of MTORC1, thereby inducing MTORC1 activation and subsequent phosphorylation of its downstream targets eukaryotic translation initiation factor 4E-binding protein 1 (EIF4EBP1, also called 4EBP1) and ribosomal protein $\mathrm{S} 6$ kinase, 70kDa, polypeptide 1 (PRPS6KB1, also called p70S6K) [67]. The increased AKT activation in the limb muscle of cachectic 


\section{CHAPTER 2}

patients compared with non-cachectic COPD patients is paralleled by an increase in phosphorylation of the downstream targets 4E-BP1 and p70S6K [44]. P70S6K phosphorylation is unaltered in COPD patients compared with controls [44], while ribosomal protein S6 (RPS6 referred to as S6) phosphorylation was even decreased in COPD patients [45]. Together, these studies show an increase in protein synthesis signaling in the limb muscles of cachectic COPD patients compared with noncachectic COPD patients, but no alteration in the general COPD population. In patients with lung cancer-related cachexia, AKT activation is increased without concurrent activation of MTOR or its downstream targets [41]. This may indicate that, although impaired AKT signaling is found in lung cancer cachexia, AKT signaling is largely intact in COPD-induced muscle wasting. However, one limitation of these studies concerns the evaluation of the activation status of protein synthesis signaling solely in a basal state. Although the current data may suggest that the protein synthesis pathway is a promising target for the treatment of COPD-induced muscle wasting, the integrity of the anabolic response should be further addressed.

Only limited data are available on anabolic signaling in respiratory muscles of COPD patients, and although the results also point to an increase in anabolic signaling, it remains unclear if this is different between cachectic and non-cachectic COPD patients. Martinez-Llorens et al. found an increase in IGF1 mRNA expression in the intercostal muscles of patients with COPD [68]. Doucet et al. compared the ratio of quadriceps to diaphragm AKT activation in COPD patients with controls and found a lower ratio in COPD [20]. This implicates that the AKT activation is relatively higher in the diaphragm than in the quadriceps. In line with this, the p70S6K phosphorylation is relatively higher in the diaphragm, while 4E-BP1 phosphorylation is higher in the quadriceps [20].

Interestingly, Tannerstedt et al. showed a difference in anabolic response between type-I and type-II muscle fibers, with increased AKT phosphorylation and downstream pathway activation in the type-II fibers [69]. In contrast, in COPD, the respiratory muscle with a shift towards more type-I fibers displayed a larger AKT activation and downstream signaling than the limb muscles with a shift towards more type-ll fibers. Therefore, the shift in fiber type does not explain the variation in AKT phosphorylation and downstream signaling between the limb muscle and respiratory muscle. Other discriminating factors, such as the muscle activity level, may be more closely linked to differences in AKT phosphorylation.

Taken together, anabolic signaling is increased in the skeletal muscle of patients with 
COPD, with an even larger increase in the diaphragm than the limb muscles. One may speculate that the increased activation of AKT signaling in the respiratory muscles is an attempt to preserve respiratory function by compensating catabolic triggers, although it may also reflect intrinsic alterations in muscle fiber composition.

\section{Myonuclear turnover}

Besides the turnover of proteins, the turnover of myonuclei appears essential for muscle regeneration [70-72]. Furthermore, although at a lower rate, myonuclear turnover might be indispensable for the maintenance of skeletal muscle mass. The regulated loss of a nucleus may involve apoptosis. Increased apoptosis, as determined by an increase in terminal deoxynucleotidyl transferase dUTP nick end labelling (TUNEL) staining and poly (ADP-ribose) polymerase cleavage in the skeletal muscle of cachectic COPD patients has been reported [73]. In the diaphragm of COPD patients with muscle wasting, elevated caspase-3 levels indicated an increase in apoptosis [55]. No difference in apoptosis measured by TUNEL staining or caspase-3 was found in COPD patients with maintained muscle mass compared with controls [74]. A possible alteration in apoptosis in the muscle of COPD patients remains inconclusive due to the lack of studies and the use of different markers. Furthermore, the possible role of apoptotic signaling in the skeletal muscle atrophy remains obscure [75], and multinucleated muscle fibers may utilize other mechanisms for selective myonuclear loss, such as autophagy [76].

To replenish the myonuclear pool, satellite cells are essential [72]. In contrast to muscle wasting in ageing $[77,78]$, the number of satellite cells per muscle fiber is unaltered in the limb and respiratory muscles of patients with COPD compared with controls $[68,79-81]$. Furthermore, no differences in satellite cell number have been reported between muscle-wasted and non-muscle wasted COPD patients [80].

Upon activation, satellite cells proliferate, differentiate, and fuse with myofibers. Activation and proliferation of satellite cells in the limb muscles does not seem to differ between COPD patients and age-matched controls based on number of satellite cells $24 \mathrm{~h}$ after a resistance-exercise bout [81]. However, molecular markers of satellite cell activation may be more sensitive than satellite cell number to quantify the satellite cell response.

In a basal condition, myogenic factor 5 mRNA expression is unaltered in the limb muscles of patients with COPD compared with controls [57]. Myogenic differentiation 1 (MYOD1) mRNA expression [40] and protein expression appear similar in the limb 


\section{CHAPTER 2}

muscles of COPD patients and healthy controls [57]. Furthermore, Myogenin (MYOG) mRNA expression is unaltered in COPD patients [57]. However, in cachectic COPD patients, MYOD1 [40] and MYOG [38, 39] protein expression are reduced compared with controls, while no alteration in MYOG protein expression was found in the limb muscles of cachectic compared with non-cachectic COPD patients [38]. It should be considered that in different populations and disease stages, the course of the satellite cell response might be altered, which may have implications for the timing of the measurements.

A negative regulator of myogenesis is Myostatin (MSTN) [82]. Limb muscle MSTN mRNA expression is increased in COPD compared with controls [57, 83], while no difference seems to be present between cachectic and non-cachectic COPD patients [40]. The increased MSTN mRNA expression in COPD patients may be partially explained by smoking status, as this increase is also found in smokers [58]. MSTN protein expression is unaltered in the limb muscle of COPD patients, independent of the pulmonary phenotype [38-40], while the circulatory level of MSTN is increased in COPD compared with control subjects [84]. It should be noted that Snijders et al. showed a delayed response in MSTN protein levels upon a single bout of resistance exercise in elderly, while basal levels did not seem to differ [85]. This implies that in the absence of a myogenic trigger, intrinsic alterations in satellite cell plasticity responses in muscle of COPD patients may be masked.

Centrally localized myonuclei in myofibers are considered derived from newly fused satellite cells prior to their final location peripherally in the myofiber against the sarcolemma. COPD patients with preserved muscle mass have higher amounts of central nuclei in the limb muscle than muscle-wasted COPD patients and controls [80]. This could be interpreted as an attempt to counteract atrophic signaling in COPD patients, which may be essential to preserve muscle mass. However, as there is only indirect evidence that central myonuclei reflect recent regenerative events, central nuclei could also reflect an increase in myonuclear turnover to compensate for increased loss of myonuclei.

To gain further insight in the regulation of myonuclear turnover and possible defects in COPD-induced skeletal muscle wasting, it is essential to incorporate satellite cell activation stimuli and sensitive techniques to monitor myonuclear accretion and turnover in the study design. This will require further development of new techniques, in parallel with novel ex vivo and in vitro approaches to monitor myonuclear accretion and possibly myonuclear loss, and assessment of the role of alterations in myonuclear turnover in muscle atrophy. 


\section{Loss of muscle oxidative phenotype}

Besides the importance of the muscle quantity for muscle function, the quality of the muscle should also be considered. This is highlighted by the finding that muscle massspecific muscle strength and endurance are reduced in patients with COPD [86-88]. A well-established qualitative alteration in the skeletal muscle of COPD patients is the loss of oxidative phenotype (OXPHEN) characterized by a muscle fiber type I to type II shift and a loss of oxidative capacity $[20,88,89]$. The loss of OXPHEN is associated with increased oxidative stress [88,90], which may render the muscle more susceptible to muscle atrophy [38]. In addition, type II fibers are generally more susceptible to atrophy stimuli including, for example, inflammation [21] and hypoxia [22]. Therefore, the loss of OXPHEN in COPD may accelerate the loss of muscle mass, thereby linking muscle quality to muscle quantity. This is supported by the fact that non-symptomatic smokers already exhibit reduced mitochondrial capacity and a similar fiber-type shift [91]. Although less extensively investigated, striking similarities are reported regarding muscle oxidative metabolism in chronic heart failure [92]. As these patients also share other systemic features and lifestyle characteristics (e.g. muscle wasting and low physical activity level) comparative analyses between well-phenotyped patients with COPD and chronic heart failure may provide more insight in common and diseasespecific denominators and mechanisms.

\section{Therapeutic perspective}

Because muscle wasting may result from alterations in the protein and myonuclear turnover, targeting key pathways in these processes will be required to combat muscle wasting.

Ubiquitin proteasome system activity is increased in the muscles of cachectic COPD patients, which implicates the atrogenes MURF1 and ATROGIN1 as targets to normalize UPS activity. This is supported by the finding that in a cell culture model and in a mouse model of muscle disuse, MURF1 inhibition and knockout, respectively, prevented muscle fiber atrophy $[93,94]$. Pharmacological inhibitors that target specific ubiquitin-conjugating and deconjugating enzymes are being developed to treat cancer, neurodegenerative disorders, and autoimmune diseases [95], but may also be highly relevant for the treatment of COPD-induced muscle wasting. Furthermore, exercise training may attenuate MURF1 expression, as was observed in the skeletal muscle of chronic heart failure patients $[50,96]$. In contrast to exercise training, one bout of exercise leads to an increase in MURF1 expression, albeit blunted in COPD [97, 98], while the increase in proteolytic signaling is reduced by branched-chain amino acid supplementation in a healthy population [97]. 


\section{CHAPTER 2}

Autophagy is disturbed in patients with COPD, although it remains unclear whether there is an increased induction of autophagy or an inhibition of autophagic-lysosomal degradation. Low amino acid availability can activate autophagy by inhibition of MTOR [99]. In line with this, branched-chain amino acid supplementation leads to an inhibition of autophagy by activation of MTOR [100]. Furthermore, overall low energy status, DNA damage, and hypoxia can inhibit MTOR through AMPK and hypoxia inducible factor 1, alpha subunit (HIF1-a) [101, 102] and thereby induce autophagy. Interestingly, exercise targets these factors, and exercise training results in elevated levels of basal autophagy [103]. However, exercise leads to an increase in muscle mass and strength in COPD patients [98]. Moreover, autophagy is required for muscle adaptations to training $[103,104]$. The counterintuitive effect of exercise on autophagy may therefore be more tightly linked to its function in quality control.

A more speculative thought is that autophagy may play a role in selective removal of damaged myonuclei. Besides causing mitochondrial DNA (mtDNA) damage, oxidative stress also causes nuclear DNA damage. COPD patients display elevated levels of oxidative stress, which may lead to increased DNA damage and requires increased removal of damaged nuclei. Although exercise induces oxidative stress, which is even accentuated in COPD patients [105], exercise also triggers myogenesis [106]. In COPD, this myogenic response to exercise seems intact, although it is specifically impaired in cachectic COPD $[40,98]$. Exercise-induced satellite cell activation is mediated by IGF signaling [107]. In contrast, MSTN signaling inhibits satellite cell activation [108]. Because MSTN expression is increased in COPD, pharmacological inhibition of MSTN might be beneficial to prevent COPD-induced muscle wasting. This idea is supported by the finding that in mice with chronic kidney failure, pharmacological inhibition of MSTN blocks muscle atrophy [109], and that pharmacological inhibition of the Actllreceptor, which mediates MSTN signaling, prevents glucocorticoid-induced muscle atrophy [110]. In vitro, glutamine reduced the tumour necrosis factor alpha-dependent increase of MSTN [111]. This implicates that availability of amino acids is important for normal satellite cell function in COPD and that restoration of normal amino acid levels may be required for muscle maintenance. Taken together, the dual function and differential regulation of UPS and autophagy in the maintenance of muscle mass and quality reflects a highly interactive signaling network that is regulated by several upstream pathways. The effect of specific modulation of UPS and autophagy mediators may therefore transcend catabolic signaling and may affect a range of other cellular processes, yielding it difficult to predict long-term side effects. So far, exercise seems to be the only intervention that can target UPS and autophagy leading to improved quantity, as well as an improved quality of the muscle in COPD patients. 
One prerequisite is that COPD patients, and specifically cachectic COPD patients, have maintained responsiveness to exercise stimuli, which remains to be established. Exercise capacity in COPD may be limited by impaired pulmonary function, leading to incapability to supply a sufficiently strong exercise trigger to the muscles. In this case, pharmacological or nutritional activators of AMPK, sirtuin 1, and peroxisome proliferator-activated receptors such as Metformin, Resveratrol, Rosglitazone, and polyunsaturated fatty acids could be used as exercise mimetics and may help sensitize the muscle to a following exercise bout. Furthermore, anabolic steroids could be considered in the treatment of COPD-induced muscle wasting, although a recent meta-analysis showed that exercise capacity of COPD patients was not improved [112]. It should also be considered that an appropriate nutritional status is necessary for the beneficial effects of exercise and that exercise (in particular, endurance type of exercise) in a malnourished state could even have detrimental effects by worsening the energy imbalance. Taken together, a multi-modal approach may be required to combat COPD-induced muscle wasting, in which exercise training is central. However, to establish such intervention, further research is crucial to determine whether the response to exercise is intact or if specific defects occur in cachectic patients with COPD.

\section{Putative mechanisms involved in a disturbed energy balance in COPD}

Specific loss of muscle mass in weight-stable COPD patients has been observed, which may reflect a tissue-specific sensitivity to an overall catabolic state. A net catabolic state may also result from an imbalance in energy expenditure and energy availability (energy balance).

\section{Increased energy expenditure}

It is well established that components of whole body energy expenditure may be increased in patients with COPD [113]. Total daily energy expenditure (TEE) is the sum of resting energy expenditure (REE), activity-induced energy expenditure (AEE), and diet-induced thermogenesis (DIT). Assessment of TEE requires sophisticated methodology including a respiration chamber [114] or doubly labelled water to allow assessment of TEE in free living conditions $[115,116]$. Data on TEE in COPD are scarce and sometimes contradicting, which may be related to the use of different methodology or patient characteristics. Based on the available evidence, it seems that in particular the emphysematous phenotype is prone to increased TEE, as high values are more often observed in patients with low carbon monoxide diffusing capacity [117-119]. This is in line with a positive effect of lung volume-reduction 


\section{CHAPTER 2}

surgery on body weight in emphysema, although surgical intervention might not only decrease energy requirements but also improve dietary intake by alleviating dyspnea [120]. More research is needed to assess putative differences in whole-body energy metabolism and its components among COPD phenotypes under comparable standardized circumstances.

Numerous studies have shown that REE is raised [121-123]. This is more prevalent in emphysema [124, 125], during acute exacerbations [126], and appears inversely correlated with forced expiratory volume in $1 \mathrm{~s}$ when comparing different studies [118, $119,122,127]$. Highest values are found among weight-losing patients [122]. This is in contrast with non-pathology-induced malnutrition, where subjects with low BMI have lower REE due to hypometabolic adjustments [128]. The same results are found for non-small-cell lung cancer (NSCLC), where REE is found to be unregulated in $74 \%$ of primary lung cancer patients [129-131]. Hypermetabolism at rest was also found to be more pronounced in weight-losing compared with weight-stable lung cancer patients [132]. Thus, increased REE is a consistent feature of chronic and more acute cachexia and seems to be more pronounced in the emphysematous subtype.

Activity-induced energy expenditure is the most variable component of TEE, and it has been postulated that COPD patients reduce physical activity to compensate for dyspnea severity or to anticipate to breathlessness. Indeed, lower physical activity levels are seen in COPD [133]. Physical inactivity is associated with advanced disease stage [134-136], exacerbations [137, 138], and degree of emphysema [139]. In addition, lung volume-reduction surgery in patients with severe emphysema improved exercise performance due to reduced lung hyperinflation, less dyspnea severity, and less cost of breathing [140]. However, it did not cause augmentation of physical activity level, implying other factors play a role, including motivation or anxiety [141]. There are several indications that when COPD patients perform physical activities, they require more energy. For example, Lahaije et al. found a higher daily activity-related oxygen consumption assessed by a face mask measuring ventilatory and metabolic demand in COPD patients compared with healthy controls [142], while Vaes et al. found an increase in FFM adjusted oxygen consumption in COPD patients compared with controls, although total oxygen consumption was not altered [143]. Indirect evidence for altered AEE is a rise in plasma ammonia in COPD patients during low intensity walking, which is an indicator of muscle ATP depletion and metabolic stress [144]. These collective data may indicate that COPD patients use oxygen less efficiently and exhibit an altered energy metabolism during physical activity. This is not surprising in view of the shift in lower limb muscle fiber type composition in COPD towards 
less oxidative fibers, which appears to be more pronounced in the emphysematous phenotype [18]. The opposite shift in muscle fiber type of the diaphragm relative to the limb muscle $[47,48]$ indicates an adaptation to chronic increase in work of breathing. Together with hyperinflation-induced mechanical inefficiency, this muscle fiber type shift could contribute to enhanced oxygen cost of breathing, illustrated by the effects of lung volume-reduction surgery [145] and non-invasive positive-pressure ventilation therapy [146]. For comparison, in lung cancer, physical activity level assessed by accelerometery is also reduced $[147,148]$, but no specific data about AEE is available.

Diet-induced thermogenesis represents metabolic oxygen cost for processing of ingested nutrients. Green and colleagues [125] indeed reported enhanced DIT in emphysematous COPD patients, but this was not confirmed by other authors [149, 150], independently of BMI [151]. These differences may be due to different test meal composition and portion size. Although oxygen desaturations during meals was noticed in severe COPD patients [152], it is unknown whether this is DIT related or not. Therefore, the thermic effect of dietary intake remains unclear. Taken together, it indicates that energy requirements are increased in COPD, and there is certainly no adaptive reduced energy demand.

In addition to the hypermetabolic state, early clinical trials have shown that enhanced systemic inflammation is a contributing factor to elevated REE, both in COPD [153] and in lung cancer [154], the source of which is yet unclear. Besides pulmonary inflammation [155], also adipose tissue has also been suggested to contribute to a higher inflammatory gene expression in adipose tissue, as has been reported in malnourished patients with advanced COPD [156].

\section{Adipose tissue metabolism}

In cachexia, muscle wasting is accompanied by loss of adipose tissue [14, 157]. In fact, in cancer-induced cachexia, adipose tissue is often one of the first affected organs, illustrated by decreasing fat cell volume and upregulation of fatty acid metabolism [158]. Regarding COPD, low BMI [11] and fat mass depletion [14] particularly occur in those with advanced disease and in the emphysematous phenotype.

Schols et al. observed low leptin levels in blood of patients with emphysema compared with chronic bronchitis in line with a lower BMI and fat mass [159]. After adjustment for FM and oral corticosteroid use as possible confounders, leptin was associated with systemic inflammation, in particular in the emphysematous patients. More recently, Brusik et al. investigated serum levels and adipose tissue expression of leptin and 


\section{CHAPTER 2}

adiponectin in patients with COPD and reported an association between decreased serum and tissue leptin levels, and decreased serum adiponectin and increased REE adjusted for body weight in underweight patients [160]. In adipose tissue two cell types can be distinguished: white adipose tissue (WAT) and brown adipose tissue (BAT). Brown adipose tissue is differentiated from WAT by the presence of cold and diet-induced thermogenesis. Thermogenesis is facilitated by BAT-specific uncoupling proteins (UCP) that dissipate the proton gradient in mitochondria in order to generate heat [161]. High amounts of mitochondria and high vascularization are responsible for the brown color of BAT [162]. Additionally, WAT can be converted in BAT, called WAT browning [163]. BAT activation negatively correlates with BMI, as demonstrated by decreased BAT activation in obese subjects [164] and during ageing [165]. Cachexia on the other hand is characterized by fat mass depletion. This raises the question whether there is a role of BAT activity in the hypermetabolic state, as seen in pulmonary cachexia.

No studies are performed to determine BAT activity in COPD patients. With respect to lung cancer, results are conflicting and scarce. Despite negative results of BAT activation reported by some authors $[166,167]$, Shellock and colleagues provided evidence for BAT activation as a cachexia mediator. Autopsy reports of cachectic cancer patients revealed high incidence of BAT in this group compared with age-matched controls [168]. Furthermore, a correlation between BAT activity and neoplastic status has been suggested [169], although the authors also reported high amount of BAT activation in non-malignancy subjects.

There is indirect evidence that BAT activation might be a potential cachexia driver in COPD as well. Hypoxia [170] and hypermetabolism [122] are hallmarks of COPD. In response to hypoxia, cells can produce vascular endothelial growth factor (VEGF) in order to restore oxygen supply [171]. This has been established by Van den Borst et al., who found an upregulation of the VEGF gene in adipose tissue in response to chronic hypoxia in mice. Congruently, adipose tissue showed a brown appearance. This browning of adipose tissue was established by increased expression of UCP1 [172], which proposes a link between hypoxia-induced VEGF activation and browning. Indeed, Sun et al. revealed upregulation of UCP-1, the main characteristic of BAT, in a VEGF overexpressing mouse model [173]. In addition, recently, increased thermogenesis and energy expenditure were found in mice with VEGF overexpression in BAT and WAT [174].

Another hypoxia alignment occurs in the form of lactate. In peripheral muscle of COPD patients, increased glycolysis metabolism is observed [175], which in turn causes 
rising lactate levels $[176,177]$. Lactate is indeed increasingly released by adipocytes in a hypoxic environment [178], which in turn is able to control the expression of UCP1. The UCP-1 regulation is independent of HIF-1a and thereby also promotes WAT browning under normoxic circumstances [179].

Another possible browning factor is beta-adrenergic stimulation mediated by norepinephrine [180, 181]. Emphysematous COPD patients indeed exhibit increased plasma norepinephrine levels [182], indicating a possible activation of the autonomic nervous system. However, Schiffelers et al. showed a blunted beta-adrenoceptormediated lipolytic and thermogenic response [183], suggesting desensitization. Additionally, in 10 lean healthy men, BAT activity in response to a systemic nonselective beta-agonist was not enhanced [184]. In contrast, blocking the receptors by propranolol decreases BAT activity [185]. Therefore, activation of brown fat through beta-adrenergic stimulation remains disputable.

It can be concluded that there is some indirect evidence pointing towards a role of BAT in pulmonary cachexia, but this area requires more research to identify therapeutic potential.

\section{Compromised dietary intake}

In order to compensate for increased energy requirements in COPD, patients should be able to adapt their dietary intake. Systematic analyses of dietary intake in COPD patients are rare. In terms of caloric content, dietary intake was found to be normal compared with healthy controls, but inadequate for measured energy expenditure [118, 186-188]. During severe acute exacerbations, the gap between energy intake and energy expenditure becomes even wider, which slowly decreases upon recovery $[126,189]$. To our knowledge, no human studies have systematically investigated the relation between dietary intake and disease severity or putative differences between emphysematous and non-emphysematous patients. Advanced disease stages and acute exacerbations are often characterized by chronic or acute hypoxemia [170]. It is well established that mice under chronic hypoxic circumstances experience weight loss, which is partly due to temporarily decreased dietary intake [172].

\section{Anorexia}

It is acknowledged that apparently normal dietary intake in COPD patients may be insufficient to meet elevated energy requirements, but reduced food intake may also be caused by anorexia, that is, loss of appetite [190]. A few underlying causes have 


\section{CHAPTER 2}

been mentioned, including nicotine use [191], physical discomfort such as dyspnea and increased breathing effort [192], depression, and anxiety [193], seen in COPD [194] as well as in NSCLC $[195,196]$.

Besides pulmonary and psychological symptoms, COPD patients often experience pain. In a Norwegian study, which controlled for age and gender, $45 \%$ of the COPD patients reported chronic pain, compared with $34 \%$ of the general population [197]. Opioids are commonly used to combat pain in COPD [198]. Side-effects of opioids occur regularly, and opioids are able to cause gastrointestinal motility disorders [199], of which constipation is the most common [200]. People suffering from constipation often present with anorexia [201], probably due to early satiety. Separate from use of pain medication, early satiety and abdominal bloating is highly prevalent in COPD [202].

\section{Chemosensory alterations}

Food intake is regulated by taste and smell [203,204], and chemosensory dysfunction could influence dietary intake. Nordén et al. showed that 21 out of 169 stable COPD patients reported taste changes, which contributed to a decreased energy intake [205]. In addition, Dewan et al. compared 20 COPD subjects with long-term oxygen therapy to 20 COPD patients without oxygen therapy and 20 healthy elderly controls. They found reduced smell and taste test scores among COPD patients compared with controls, independent of oxygen supply [206]. Also, Wardwell and colleagues found that healthy elderly tended to be able to identify more different tastes correctly than COPD patients, although not statistically significant [207]. Both authors did not report medication use, and therefore, the influence of treatment is unknown. Although data are scarce and methodological quality of the studies is limited, these data suggest that COPD or its treatment could modify taste and smell detection.

\section{Food reward system}

Fullness is regulated by gastrointestinal hormones, including leptin and ghrelin [208], and their secretion is affected by dietary intake and nutritional status. Clinically stable emphysematous COPD patients exhibit low leptin levels compared with the chronic bronchitis subtype [159]. During acute flare-ups, these plasma levels rise temporary [189], as seen in NSCLC [209, 210]. Likewise, enhanced plasma ghrelin levels are noticed in COPD [211] and NSCLC [212], and are related to cachectic status.

The peripheral hormonal satiety system closely interacts with the central nervous 
system in order to regulate food intake. Brain imaging studies have revealed rewardspecific brain regions related to food reward [213], and activation of these regions correlate with food rewarding [214]. Different orexigenic and anorexigenic peptides and hormones can stimulate neurons in these specific cerebral regions [208, 215]. For instance, leptin inhibits neurons, causing reduced food intake and increased energy expenditure [216]. Ghrelin, considered to be a leptin counterpart, can induce food intake mediated by stimulation of neurons in this area [217].

There is surprisingly no human study available that explored the role of central dysregulation in food reward in patients with COPD. In relation to lung cancer cachexia, only one study was performed identifying brain activity in anorectic and non-anorectic patients while receiving pleasant and unpleasant food cues [218]. In contrast to nonanorectic patients, anorectic patients showed no brain activity differences in response to pleasant versus unpleasant pictures. This implies an overall blunted response in the perceptual and motivational system that could also be involved in COPD but requires further investigation.

\section{Therapeutic perspective}

The importance of nutritional status is not only emphasized by adverse effects on muscle function and exercise performance but also by detrimental effects of malnutrition on lung tissue. These effects have mostly been studied in animal models. Following the clinical phenotyping of the pink puffer and the blue bloater in the 1960s, Sahebjami et al. found reinforcement of pre-existing emphysematous processes due to caloric food deprivation in rats [219], which was more pronounced in young rats [220]. These deleterious effects could partly be reversed by refeeding [221]. In contrast, Bishai et al. found no alveolar size changes in calorie-restricted mice, although the lungs became stiffer and lung capacity was decreased [222]. Supplementary evidence was provided by emphysema-like changes present in anorexia nervosa patients, which underscores the impact of chronic malnutrition on alveoli [223]. In addition to lung tissue, respiratory muscles also contribute to breathing. Weight loss does not spare the respiratory muscles, because weight loss is related to diminished diaphragm weight [224] and decreased function [225] in experimental models and in humans.

As proof of concept, Efthimiou et al. conducted a randomized controlled trial to investigate the effect of nutritional support on respiratory and peripheral muscle function in malnourished COPD patients. They reported improvement in respiratory muscle strength and hand grip strength, accompanied by less dyspnea and enhanced distance in 6-min walk test. Importantly, these effects diminished after quitting the 


\section{CHAPTER 2}

dietary supplementation [226]. The positive effects of dietary support on body weight was verified by Weekes et al., who found weight gain in the intervention group, whereas the control group continued to lose weight. Addition of dietary counselling to dietary support has been shown to maintain weight loss after cessation of intervention [227].

Initially, the focus was primarily on caloric intake to balance energy requirements, but more recent proof of concept experiments also highlighted the importance of optimizing protein intake [228, 229]. Low intake of other essential nutrients is identified, including vitamin D and calcium [230], which are also relevant in the context of osteoporosis as clustering comorbid condition.

One should keep in mind that dietary intake does not reflect actual availability of ingested micronutrients. There are indications that intestinal function is impaired in COPD, illustrated by splanchnic hypoperfusion and reduced intestinal permeability [231]. Altered intestinal function translates into reduced splanchnic extraction of amino acids derived from nutritional intake [36, 232], but as a result, the amino acid uptake in the skeletal muscle of clinically stable COPD patients appears increased [233]. Thus, the significance for clinical applications remains ambiguous.

Both dietary intake and nutrient availability are controlled by gastrointestinal hormones. By binding to the growth hormone secretagogue receptor, ghrelin can induce secretion of growth hormone [234]. This leads to modulation of the growth hormone/IGF1 axis, which is an important anabolic pathway in human skeletal muscle [235]. Furthermore, ghrelin can induce food intake, mediated by stimulation of specific neurons in the food reward center [217]. Due to its orexigenic property, ghrelin analogues have been proposed for clinical application in cachexia. One clinical trial with ghrelin analogues have been conducted in COPD patients. They reported improvements of ventilatory efficiency at peak exercise, reflected by increased peak oxygen uptake [236]. However, it did not translate in improved 6-min walk distance, and no data are available about body composition or food intake. Clinical trials in cancer cachexia [237], including lung cancer [238], demonstrate an enhanced lean body mass and quality of life. Hence, ghrelin analogues warrant further investigation in COPD. Besides dietary and pharmacological interventions, cognitive behavioral interventions are relatively underexplored in the management of cachexia in COPD. Although results from different functional neuroimaging studies are inconsistent and sometimes conflicting [239], there might be altered reactivity in the brain reward system in response to perceived food stimuli in people with altered eating patterns, including anorexia nervosa and obesity $[240,241]$. Therefore, cognitive behavioral 
therapy may serve as a treatment for patients with an eating disorder like anorexia nervosa [242]. Recently, a randomized controlled trial was conducted in obese subjects, receiving behavioral therapy for 6 months in order to reduce weight. Analysis of functional magnetic resonance imaging revealed changes in reward system activity in the intervention group versus controls. Further research has to identify whether it is possible to enhance neuroplasticity in the food reward center in order to increase successfulness for eating disorder treatment [243]. This opens up new insights and therapeutic opportunities for suspected nutritional therapy-resistant cachectic COPD patients, if disturbances in the central food reward system are indeed identified.

\section{Conclusions}

It is well established that the prevalence and related disease burden of cachexia is high in COPD and likely to increase in the near future given the high and increasing prevalence of the disease in an ageing population. Nevertheless, cachexia management is still poorly implemented in clinical practice. In 2014, the European Respiratory Society published a statement on nutritional assessment and therapy in COPD including a nutritional risk stratification diagram based on assessment of BMI, weight changes, and body composition, which could be useful in patient counselling [24]. In order to increase overall survival and compress morbidity, a multi-modal intervention approach is needed, which should target the discussed factors involved in cachexia (Figure 1). Such a multi-modal intervention approach, encompassing exercise training and improvement of energy balance and nutrient availability, is currently feasible as supported by recent statements and meta-analyses, possibly improved in the near future by targeted pharmacological interventions and cognitive behavioral therapy to sensitize patients to anabolic stimuli. 


\section{CHAPTER 2}
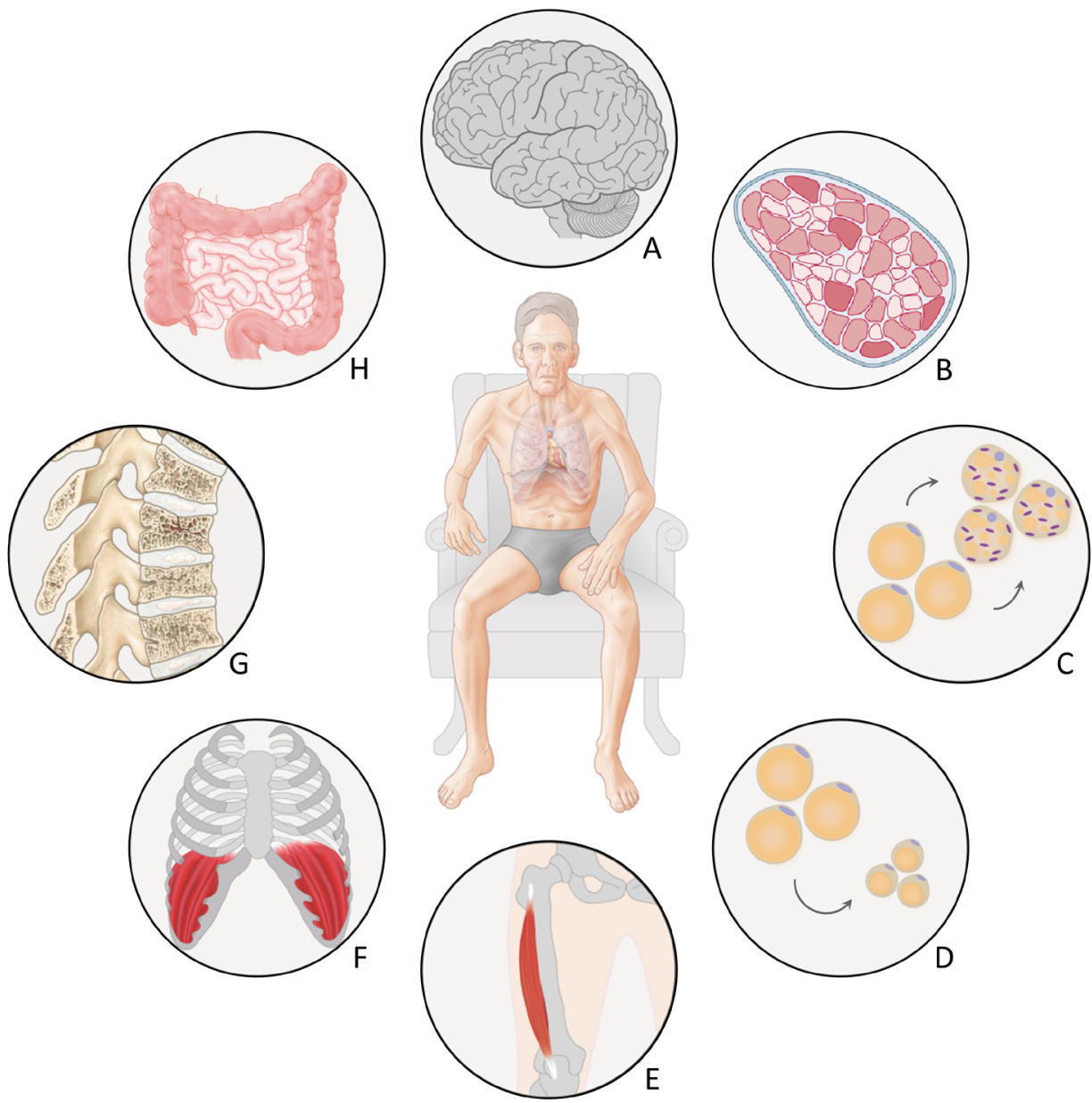

Figure 1. | Pulmonary and extra-pulmonary cross-talk in COPD cachexia. A. Altered brain responses to food stimuli. B. Muscle fiber type shifting and oxidative metabolism. C. Altered adipose tissue metabolism. D. Adipose tissue wasting. E. Limb muscle dysfunction. F. Respiratory muscle dysfunction. G. Osteoporosis. H. Altered gut integrity and reduced splanchnic extraction 


\section{REFERENCES}

1. Mathers CD, Loncar D. Projections of global mortality and burden of disease from 2002 to 2030. PLoS medicine 2006;3(11):e442.

2. Prince $M J, W u F, G u o ~ Y$, et al. The burden of disease in older people and implications for health policy and practice. Lancet 2014 [published Online First: 2014/12/04]

3. Vestbo J, Hurd SS, Agusti AG, et al. Global strategy for the diagnosis, management, and prevention of chronic obstructive pulmonary disease: GOLD executive summary. Am J Respir Crit Care Med 2013;187(4):347-65.

4. Vanfleteren LE, Spruit MA, Groenen M, et al. Clusters of comorbidities based on validated objective measurements and systemic inflammation in patients with chronic obstructive pulmonary disease. Am J Respir Crit Care Med 2013;187(7):728-35.

5. Burgel PR, Paillasseur JL, Peene B, et al. Two distinct chronic obstructive pulmonary disease (COPD) phenotypes are associated with high risk of mortality. PloS one 2012;7(12):e51048. [published Online First: 2012/12/14]

6. Romme EA, Murchison JT, Phang KF, et al. Bone attenuation on routine chest $\mathrm{CT}$ correlates with bone mineral density on DXA in patients with COPD. J Bone Miner Res 2012;27(11):233843. [published Online First: 2012/06/14]

7. Schols AM, Soeters PB, Dingemans AM, et al. Prevalence and characteristics of nutritional depletion in patients with stable COPD eligible for pulmonary rehabilitation. Am Rev Respir Dis 1993;147(5):1151-6. [published Online First: 1993/05/01]

8. Vestbo J, Prescott E, Almdal T, et al. Body mass, fat-free body mass, and prognosis in patients with chronic obstructive pulmonary disease from a random population sample: findings from the Copenhagen City Heart Study. Am J Respir Crit Care Med 2006;173(1):79-83.

9. Mostert R, Goris A, Weling-Scheepers $C$, et al. Tissue depletion and health related quality of life in patients with chronic obstructive pulmonary disease. Respir Med 2000;94(9):859-67.

10. Engelen MP, Schols AM, Baken WC, et al. Nutritional depletion in relation to respiratory and peripheral skeletal muscle function in out-patients with COPD. Eur Respir J 1994;7(10): 17937.

11. Schols AM, Broekhuizen R, Weling-Scheepers CA, et al. Body composition and mortality in chronic obstructive pulmonary disease. The American journal of clinical nutrition 2005;82(1):53-9.

12. Bernard $S$, LeBlanc $P$, Whittom $F$, et al. Peripheral muscle weakness in patients with chronic obstructive pulmonary disease. Am J Respir Crit Care Med 1998;158(2):629-34. [published Online First: 1998/08/12]

13. Engelen MP, Schols AM, Does JD, et al. Skeletal muscle weakness is associated with wasting of extremity fat-free mass but not with airflow obstruction in patients with chronic obstructive pulmonary disease. Am J Clin Nutr 2000;71(3):733-8. [published Online First: 2000/03/04]

14. Engelen MP, Schols AM, Lamers RJ, et al. Different patterns of chronic tissue wasting among patients with chronic obstructive pulmonary disease. Clin Nutr 1999;18(5):275-80. [published Online First: 1999/12/22]

15. Caron MA, Debigare R, Dekhuijzen PN, et al. Comparative assessment of the quadriceps and the diaphragm in patients with COPD. Journal of applied physiology 2009;107(3):952-61. [published Online First: 2009/04/11]

16. Gosker HR, Engelen MP, van Mameren $\mathrm{H}$, et al. Muscle fiber type IIX atrophy is involved in the loss of fat-free mass in chronic obstructive pulmonary disease. Am J Clin Nutr 2002;76(1):113-9. [published Online First: 2002/06/26]

17. Mancini DM, Coyle E, Coggan A, et al. Contribution of intrinsic skeletal muscle changes to 31P NMR skeletal muscle metabolic abnormalities in patients with chronic heart failure. 


\section{CHAPTER 2}

Circulation 1989;80(5):1338-46.

18. Gosker HR, van Mameren $H$, van Dijk PJ, et al. Skeletal muscle fibre-type shifting and metabolic profile in patients with chronic obstructive pulmonary disease. Eur Respir J 2002;19(4):617-25.

19. Maltais $F$, LeBlanc $P$, Whittom $F$, et al. Oxidative enzyme activities of the vastus lateralis muscle and the functional status in patients with COPD. Thorax 2000;55(10):848-53.

20. Remels $A H$, Gosker HR, Langen $\mathrm{RC}$, et al. The mechanisms of cachexia underlying muscle dysfunction in COPD. Journal of applied physiology 2013;114(9):1253-62.

21. Files DC, D'Alessio FR, Johnston LF, et al. A critical role for muscle ring finger-1 in acute lung injury-associated skeletal muscle wasting. Am J Respir Crit Care Med 2012;185(8):825-34. [published Online First: 2012/02/09]

22. de Theije CC, Langen RC, Lamers WH, et al. Differential sensitivity of oxidative and glycolytic muscles to hypoxia-induced muscle atrophy. Journal of applied physiology 2014:jap 00624 2014. [published Online First: 2014/11/28]

23. van de Bool C, Rutten EP, Franssen FM, et al. Antagonistic implications of sarcopenia and abdominal obesity on physical performance in COPD. Eur Respir J 2015

24. Schols AM, Ferreira IM, Franssen FM, et al. Nutritional assessment and therapy in COPD: a European Respiratory Society statement. Eur Respir J 2014

25. Jones SE, Maddocks M, Kon SS, et al. Sarcopenia in COPD: prevalence, clinical correlates and response to pulmonary rehabilitation. Thorax 2015

26. Cherin $\mathrm{P}$, Voronska $\mathrm{E}$, Fraoucene $\mathrm{N}$, et al. Prevalence of sarcopenia among healthy ambulatory subjects: the sarcopenia begins from 45 years. Aging Clin Exp Res 2014;26(2):137-46. [published Online First: 2013/10/17]

27. O'Donnell DE, Ciavaglia CE, Neder JA. When obesity and chronic obstructive pulmonary disease collide. Physiological and clinical consequences. Ann Am Thorac Soc 2014;11(4):63544.

28. Guenette JA, Jensen $D, O^{\prime}$ Donnell $D E$. Respiratory function and the obesity paradox. Current opinion in clinical nutrition and metabolic care 2010;13(6):618-24. [published Online First: 2010/10/27]

29. van den Borst B, Gosker HR, Koster A, et al. The influence of abdominal visceral fat on inflammatory pathways and mortality risk in obstructive lung disease. Am J Clin Nutr 2012;96(3):516-26. [published Online First: 2012/07/20]

30. Furutate $\mathrm{R}$, Ishii $\mathrm{T}$, Wakabayashi $\mathrm{R}$, et al. Excessive visceral fat accumulation in advanced chronic obstructive pulmonary disease. Int J Chron Obstruct Pulmon Dis 2011;6:423-30.

31. McDonald ML, Diaz AA, Ross JC, et al. Quantitative computed tomography measures of pectoralis muscle area and disease severity in chronic obstructive pulmonary disease. A cross-sectional study. Ann Am Thorac Soc 2014;11(3):326-34. [published Online First: 2014/02/25]

32. Diaz AA, Zhou L, Young TP, et al. Chest CT measures of muscle and adipose tissue in COPD: gender-based differences in content and in relationships with blood biomarkers. Academic radiology 2014;21(10):1255-61. [published Online First: 2014/08/05]

33. Langen RC, Gosker HR, Remels AH, et al. Triggers and mechanisms of skeletal muscle wasting in chronic obstructive pulmonary disease. The international journal of biochemistry \& cell biology 2013;45(10):2245-56. [published Online First: 2013/07/06]

34. Zuo L, He F, Sergakis GG, et al. Interrelated role of cigarette smoking, oxidative stress, and immune response in COPD and corresponding treatments. Am J Physiol Lung Cell Mol Physiol 2014;307(3):L205-18.

35. Rutten EP, Franssen FM, Engelen MP, et al. Greater whole-body myofibrillar protein breakdown in cachectic patients with chronic obstructive pulmonary disease. Am J Clin Nutr 2006;83(4):829-34. 
36. Engelen MP, Deutz NE, Wouters EF, et al. Enhanced levels of whole-body protein turnover in patients with chronic obstructive pulmonary disease. Am J Respir Crit Care Med 2000;162(4 Pt 1):1488-92.

37. Morrison WL, Gibson JN, Scrimgeour C, et al. Muscle wasting in emphysema. Clin Sci (Lond) 1988;75(4):415-20. [published Online First: 1988/10/01]

38. Fermoselle $C$, Rabinovich $R$, Ausin $P$, et al. Does oxidative stress modulate limb muscle atrophy in severe COPD patients? Eur Respir J 2012;40(4):851-62.

39. Puig-Vilanova E, Rodriguez DA, Lloreta J, et al. Oxidative Stress, Redox Signaling Pathways, And Autophagy In Cachectic Muscles Of Male Patients With Advanced Copd And Lung Cancer. Free Radic Biol Med 2014 [published Online First: 2014/12/03]

40. Vogiatzis I, Simoes DC, Stratakos G, et al. Effect of pulmonary rehabilitation on muscle remodelling in cachectic patients with COPD. Eur Respir J 2010;36(2):301-10.

41. Op den Kamp CM, Langen RC, Snepvangers FJ, et al. Nuclear transcription factor kappa B activation and protein turnover adaptations in skeletal muscle of patients with progressive stages of lung cancer cachexia. Am J Clin Nutr 2013;98(3):738-48.

42. Debigare $\mathrm{R}$, Cote $\mathrm{CH}$, Maltais F. Ubiquitination and proteolysis in limb and respiratory muscles of patients with chronic obstructive pulmonary disease. Proceedings of the American Thoracic Society 2010;7(1):84-90. [published Online First: 2010/02/18]

43. Debigare $\mathrm{R}$, Maltais $\mathrm{F}$, Cote $\mathrm{CH}$, et al. Profiling of mRNA expression in quadriceps of patients with COPD and muscle wasting. COPD 2008;5(2):75-84.

44. Doucet M, Russell AP, Leger B, et al. Muscle atrophy and hypertrophy signaling in patients with chronic obstructive pulmonary disease. Am J Respir Crit Care Med 2007;176(3):261-9.

45. Guo Y, Gosker HR, Schols AM, et al. Autophagy in locomotor muscles of patients with chronic obstructive pulmonary disease. Am J Respir Crit Care Med 2013;188(11):1313-20.

46. Doucet $M$, Dube A, Joanisse DR, et al. Atrophy and hypertrophy signalling of the quadriceps and diaphragm in COPD. Thorax 2010;65(11):963-70.

47. Levine S, Gregory C, Nguyen T, et al. Bioenergetic adaptation of individual human diaphragmatic myofibers to severe COPD. Journal of applied physiology 2002;92(3):120513.

48. Levine S, Kaiser L, Leferovich J, et al. Cellular adaptations in the diaphragm in chronic obstructive pulmonary disease. N Engl J Med 1997;337(25):1799-806.

49. Mofarrahi M, Guo Y, Haspel JA, et al. Autophagic flux and oxidative capacity of skeletal muscles during acute starvation. Autophagy 2013;9(10):1604-20.

50. Gielen S, Sandri M, Kozarez I, et al. Exercise training attenuates MuRF-1 expression in the skeletal muscle of patients with chronic heart failure independent of age: the randomized Leipzig Exercise Intervention in Chronic Heart Failure and Aging catabolism study. Circulation 2012;125(22):2716-27.

51. Sandri M, Sandri C, Gilbert A, et al. Foxo transcription factors induce the atrophy-related ubiquitin ligase atrogin-1 and cause skeletal muscle atrophy. Cell 2004;117(3):399-412.

52. Mammucari C, Milan G, Romanello V, et al. FoxO3 controls autophagy in skeletal muscle in vivo. Cell Metab 2007;6(6):458-71. [published Online First: 2007/12/07]

53. Glickman $\mathrm{MH}$, Ciechanover A. The ubiquitin-proteasome proteolytic pathway: destruction for the sake of construction. Physiol Rev 2002;82(2):373-428. [published Online First: 2002/03/28]

54. Pickart CM. Back to the future with ubiquitin. Cell 2004;116(2):181-90. [published Online First: 2004/01/28]

55. Ottenheijm CA, Heunks LM, Li YP, et al. Activation of the ubiquitin-proteasome pathway in the diaphragm in chronic obstructive pulmonary disease. Am J Respir Crit Care Med 2006;174(9):997-1002. 


\section{CHAPTER 2}

56. Lemire BB, Debigare $R$, Dube $A$, et al. MAPK signaling in the quadriceps of patients with chronic obstructive pulmonary disease. Journal of applied physiology 2012;113(1):159-66.

57. Plant PJ, Brooks D, Faughnan $M$, et al. Cellular markers of muscle atrophy in chronic obstructive pulmonary disease. Am J Respir Cell Mol Biol 2010;42(4):461-71.

58. Petersen AM, Magkos $F$, Atherton $P$, et al. Smoking impairs muscle protein synthesis and increases the expression of myostatin and MAFbx in muscle. Am J Physiol Endocrinol Metab 2007;293(3):E843-8.

59. Yang Z, Klionsky DJ. An overview of the molecular mechanism of autophagy. Curr Top Microbiol Immunol 2009;335:1-32.

60. Mizushima N. The role of the Atg1/ULK1 complex in autophagy regulation. Current opinion in cell biology 2010;22(2):132-9. [published Online First: 2010/01/09]

61. Mammucari $C$, Schiaffino S, Sandri M. Downstream of Akt: FoxO3 and mTOR in the regulation of autophagy in skeletal muscle. Autophagy 2008;4(4):524-6. [published Online First: 2008/03/28]

62. Mai S, Muster B, Bereiter-Hahn J, et al. Autophagy proteins LC3B, ATG5 and ATG12 participate in quality control after mitochondrial damage and influence lifespan. Autophagy 2012;8(1):47-62. [published Online First: 2011/12/16]

63. Rello-Varona S, Lissa D, Shen S, et al. Autophagic removal of micronuclei. Cell Cycle 2012;11(1):170-6. [published Online First: 2011/12/22]

64. Lewis A, Riddoch-Contreras J, Natanek SA, et al. Downregulation of the serum response factor/miR-1 axis in the quadriceps of patients with COPD. Thorax 2012;67(1):26-34.

65. Crul T, Spruit MA, Gayan-Ramirez G, et al. Markers of inflammation and disuse in vastus lateralis of chronic obstructive pulmonary disease patients. Eur J Clin Invest 2007;37(11):897904.

66. Adams GR. Invited Review: Autocrine/paracrine IGF-I and skeletal muscle adaptation. Journal of applied physiology 2002;93(3):1159-67.

67. Hay N, Sonenberg N. Upstream and downstream of mTOR. Genes \& development 2004;18(16):1926-45. [published Online First: 2004/08/18]

68. Martinez-Llorens J, Casadevall C, Lloreta J, et al. [Activation of satellite cells in the intercostal muscles of patients with chronic obstructive pulmonary disease]. Archivos de bronconeumologia 2008;44(5):239-44.

69. Tannerstedt J, Apro W, Blomstrand E. Maximal lengthening contractions induce different signaling responses in the type I and type II fibers of human skeletal muscle. Journal of applied physiology 2009;106(4):1412-8. [published Online First: 2008/12/30]

70. Lepper C, Partridge TA, Fan CM. An absolute requirement for Pax7-positive satellite cells in acute injury-induced skeletal muscle regeneration. Development 2011;138(17):3639-46.

71. Relaix F, Zammit PS. Satellite cells are essential for skeletal muscle regeneration: the cell on the edge returns centre stage. Development 2012;139(16):2845-56.

72. Adams GR, Caiozzo VJ, Haddad F, et al. Cellular and molecular responses to increased skeletal muscle loading after irradiation. Am J Physiol Cell Physiol 2002;283(4):C1182-95.

73. Agusti AG, Sauleda J, Miralles C, et al. Skeletal muscle apoptosis and weight loss in chronic obstructive pulmonary disease. Am J Respir Crit Care Med 2002;166(4):485-9. [published Online First: 2002/08/21]

74. Gosker HR, Kubat B, Schaart G, et al. Myopathological features in skeletal muscle of patients with chronic obstructive pulmonary disease. Eur Respir J 2003;22(2):280-5.

75. Dupont-Versteegden EE. Apoptosis in skeletal muscle and its relevance to atrophy. World J Gastroenterol 2006;12(46):7463-6.

76. Mijaljica D, Devenish RJ. Nucleophagy at a glance. J Cell Sci 2013;126(Pt 19):4325-30. [published Online First: 2013/09/10] 
77. Verdijk LB, Snijders T, Drost M, et al. Satellite cells in human skeletal muscle; from birth to old age. Age (Dordr) 2014;36(2):545-7.

78. Snijders T, Verdijk LB, van Loon LJ. The impact of sarcopenia and exercise training on skeletal muscle satellite cells. Ageing Res Rev 2009;8(4):328-38.

79. Eliason G, Abdel-Halim S, Arvidsson B, et al. Physical performance and muscular characteristics in different stages of COPD. Scand J Med Sci Sports 2009;19(6):865-70.

80. Theriault ME, Pare ME, Maltais F, et al. Satellite cells senescence in limb muscle of severe patients with COPD. PLoS One 2012;7(6):e39124. [published Online First: 2012/06/22]

81. Menon MK, Houchen L, Singh SJ, et al. Inflammatory and satellite cells in the quadriceps of patients with COPD and response to resistance training. Chest 2012;142(5):1134-42.

82. Rodriguez J, Vernus B, Chelh I, et al. Myostatin and the skeletal muscle atrophy and hypertrophy signaling pathways. Cell Mol Life Sci 2014;71(22):4361-71. [published Online First: 2014/08/01]

83. Man WD, Natanek SA, Riddoch-Contreras J, et al. Quadriceps myostatin expression in COPD. Eur Respir J 2010;36(3):686-8.

84. Ju CR, Chen RC. Serum myostatin levels and skeletal muscle wasting in chronic obstructive pulmonary disease. Respir Med 2012;106(1):102-8.

85. Snijders T, Verdijk LB, Smeets JS, et al. The skeletal muscle satellite cell response to a single bout of resistance-type exercise is delayed with aging in men. Age 2014;36(4):9699. [published Online First: 2014/08/12]

86. Malaguti C, Nery LE, Dal Corso $S$, et al. Scaling skeletal muscle function to mass in patients with moderate-to-severe COPD. Eur J Appl Physiol 2006;98(5):482-8.

87. Malaguti $C$, Napolis LM, Villaca $D$, et al. Relationship between peripheral muscle structure and function in patients with chronic obstructive pulmonary disease with different nutritional status. J Strength Cond Res 2011;25(7):1795-803.

88. van den Borst B, Slot IG, Hellwig VA, et al. Loss of quadriceps muscle oxidative phenotype and decreased endurance in patients with mild-to-moderate COPD. Journal of applied physiology 2013;114(9):1319-28.

89. Gosker HR, Zeegers MP, Wouters EF, et al. Muscle fibre type shifting in the vastus lateralis of patients with COPD is associated with disease severity: a systematic review and metaanalysis. Thorax 2007;62(11):944-9.

90. Barreiro E, de la Puente B, Minguella J, et al. Oxidative stress and respiratory muscle dysfunction in severe chronic obstructive pulmonary disease. Am J Respir Crit Care Med 2005;171(10):1116-24. [published Online First: 2005/03/01]

91. Degens H, Gayan-Ramirez G, van Hees HW. Smoking-induced skeletal muscle dysfunction: from evidence to mechanisms. Am J Respir Crit Care Med 2015;191(6):620-5.

92. Gosker HR, Wouters EF, van der Vusse GJ, et al. Skeletal muscle dysfunction in chronic obstructive pulmonary disease and chronic heart failure: underlying mechanisms and therapy perspectives. Am J Clin Nutr 2000;71(5):1033-47.

93. Labeit $\mathrm{S}$, Kohl $\mathrm{CH}$, Witt CC, et al. Modulation of muscle atrophy, fatigue and MLC phosphorylation by MuRF1 as indicated by hindlimb suspension studies on MuRF1-KO mice. J Biomed Biotechnol 2010;2010:693741.

94. Eddins MJ, Marblestone JG, Suresh Kumar KG, et al. Targeting the ubiquitin E3 ligase MuRF1 to inhibit muscle atrophy. Cell biochemistry and biophysics 2011;60(1-2):113-8.

95. Edelmann MJ, Nicholson B, Kessler BM. Pharmacological targets in the ubiquitin system offer new ways of treating cancer, neurodegenerative disorders and infectious diseases. Expert reviews in molecular medicine 2011;13:e35.

96. Gosker HR, Lencer NH, Franssen FM, et al. Striking similarities in systemic factors contributing to decreased exercise capacity in patients with severe chronic heart failure or COPD. Chest 


\section{CHAPTER 2}

2003;123(5):1416-24.

97. Borgenvik $\mathrm{M}$, Apro W, Blomstrand E. Intake of branched-chain amino acids influences the levels of MAFbx mRNA and MuRF-1 total protein in resting and exercising human muscle. Am J Physiol Endocrinol Metab 2012;302(5):E510-21.

98. Constantin D, Menon MK, Houchen-Wolloff L, et al. Skeletal muscle molecular responses to resistance training and dietary supplementation in COPD. Thorax 2013;68(7):625-33.

99. Hara K, Yonezawa K, Weng QP, et al. Amino acid sufficiency and mTOR regulate p70 S6 kinase and elF-4E BP1 through a common effector mechanism. J Biol Chem 1998;273(23):1448494.

100. Drummond MJ, Rasmussen BB. Leucine-enriched nutrients and the regulation of mammalian target of rapamycin signalling and human skeletal muscle protein synthesis. Current opinion in clinical nutrition and metabolic care 2008;11(3):222-6. [published Online First: 2008/04/12]

101. Ditch S, Paull TT. The ATM protein kinase and cellular redox signaling: beyond the DNA damage response. Trends in biochemical sciences 2012;37(1):15-22.

102. Sabatini DM. mTOR and cancer: insights into a complex relationship. Nature reviews Cancer 2006;6(9):729-34.

103. Lira VA, Okutsu $M$, Zhang $M$, et al. Autophagy is required for exercise training-induced skeletal muscle adaptation and improvement of physical performance. FASEB journal : official publication of the Federation of American Societies for Experimental Biology 2013;27(10):4184-93. [published Online First: 2013/07/05]

104. Sanchez AM, Bernardi H, Py G, et al. Autophagy is essential to support skeletal muscle plasticity in response to endurance exercise. Am J Physiol Regul Integr Comp Physiol 2014;307(8):R956-69.

105. Couillard A, Maltais F, Saey D, et al. Exercise-induced quadriceps oxidative stress and peripheral muscle dysfunction in patients with chronic obstructive pulmonary disease. Am J Respir Crit Care Med 2003;167(12):1664-9.

106. Wilborn CD, Taylor LW, Greenwood M, et al. Effects of different intensities of resistance exercise on regulators of myogenesis. J Strength Cond Res 2009;23(8):2179-87.

107. Hill M, Goldspink G. Expression and splicing of the insulin-like growth factor gene in rodent muscle is associated with muscle satellite (stem) cell activation following local tissue damage. J Physiol 2003;549(Pt 2):409-18.

108. Snijders T, Verdijk LB, McKay BR, et al. Acute dietary protein intake restriction is associated with changes in myostatin expression after a single bout of resistance exercise in healthy young men. J Nutr 2014;144(2):137-45.

109.Zhang L, Rajan V, Lin E, et al. Pharmacological inhibition of myostatin suppresses systemic inflammation and muscle atrophy in mice with chronic kidney disease. FASEB journal : official publication of the Federation of American Societies for Experimental Biology 2011;25(5):1653-63.

110. Lach-Trifilieff E, Minetti GC, Sheppard K, et al. An antibody blocking activin type II receptors induces strong skeletal muscle hypertrophy and protects from atrophy. Mol Cell Biol 2014;34(4):606-18.

111. Bonetto A, Penna F, Minero VG, et al. Glutamine prevents myostatin hyperexpression and protein hypercatabolism induced in $\mathrm{C} 2 \mathrm{C} 12$ myotubes by tumor necrosis factor-alpha. Amino Acids 2011;40(2):585-94.

112. Pan L, Wang $M$, Xie $X$, et al. Effects of anabolic steroids on chronic obstructive pulmonary disease: a meta-analysis of randomised controlled trials. PLoS One 2014;9(1):e84855.

113. Schols AM. Nutrition as a metabolic modulator in COPD. Chest 2013;144(4):1340-5. [published Online First: 2013/10/02]

114. Schoffelen PF, Westerterp KR, Saris WH, et al. A dual-respiration chamber system with 
automated calibration. Journal of applied physiology 1997;83(6):2064-72.

115. Roberts SB. Use of the doubly labeled water method for measurement of energy expenditure, total body water, water intake, and metabolizable energy intake in humans and small animals. Canadian journal of physiology and pharmacology 1989;67(10):1190-8.

116. Schoeller DA, Ravussin E, Schutz Y, et al. Energy expenditure by doubly labeled water: validation in humans and proposed calculation. Am J Physiol 1986;250(5 Pt 2):R823-30.

117. Hugli O, Schutz Y, Fitting JW. The daily energy expenditure in stable chronic obstructive pulmonary disease. Am J Respir Crit Care Med 1996;153(1):294-300.

118. Slinde F, Ellegard L, Gronberg AM, et al. Total energy expenditure in underweight patients with severe chronic obstructive pulmonary disease living at home. Clin Nutr 2003;22(2):15965.

119. Baarends EM, Schols AM, Westerterp KR, et al. Total daily energy expenditure relative to resting energy expenditure in clinically stable patients with COPD. Thorax 1997;52(9):780-5.

120. Mineo TC, Pompeo E, Mineo D, et al. Resting energy expenditure and metabolic changes after lung volume reduction surgery for emphysema. Ann Thorac Surg 2006;82(4):1205-11.

121. Kao CC, Hsu JW, Bandi V, et al. Resting energy expenditure and protein turnover are increased in patients with severe chronic obstructive pulmonary disease. Metabolism 2011;60(10):1449-55.

122. Schols AM, Fredrix EW, Soeters PB, et al. Resting energy expenditure in patients with chronic obstructive pulmonary disease. Am J Clin Nutr 1991;54(6):983-7. [published Online First: 1991/12/01]

123. Goldstein S, Askanazi J, Weissman C, et al. Energy expenditure in patients with chronic obstructive pulmonary disease. Chest 1987;91(2):222-4.

124. Cohen Rl, Marzouk K, Berkoski P, et al. Body composition and resting energy expenditure in clinically stable, non-weight-losing patients with severe emphysema. Chest 2003;124(4):1365-72.

125. Green JH, Muers MF. The thermic effect of food in underweight patients with emphysematous chronic obstructive pulmonary disease. Eur Respir J 1991;4(7):813-9.

126. Vermeeren MA, Schols AM, Wouters EF. Effects of an acute exacerbation on nutritional and metabolic profile of patients with COPD. Eur Respir J 1997;10(10):2264-9. [published Online First: 1997/12/05]

127. Ryan CF, Road JD, Buckley PA, et al. Energy balance in stable malnourished patients with chronic obstructive pulmonary disease. Chest 1993;103(4):1038-44.

128. Kurpad AV, Muthayya S, Vaz M. Consequences of inadequate food energy and negative energy balance in humans. Public health nutrition 2005;8(7A):1053-76.

129. Fredrix EW, Staal-van den Brekel AJ, Wouters EF. Energy balance in nonsmall cell lung carcinoma patients before and after surgical resection of their tumors. Cancer 1997;79(4):717-23.

130. Harvie MN, Campbell IT, Thatcher N, et al. Changes in body composition in men and women with advanced nonsmall cell lung cancer (NSCLC) undergoing chemotherapy. Journal of human nutrition and dietetics : the official journal of the British Dietetic Association 2003;16(5):323-6.

131. Staal-van den Brekel AJ, Schols AM, Dentener MA, et al. Metabolism in patients with small cell lung carcinoma compared with patients with non-small cell lung carcinoma and healthy controls. Thorax 1997;52(4):338-41.

132. Cao DX, Wu GH, Zhang B, et al. Resting energy expenditure and body composition in patients with newly detected cancer. Clin Nutr 2010;29(1):72-7.

133. Van Remoortel $\mathrm{H}$, Hornikx M, Demeyer $\mathrm{H}$, et al. Daily physical activity in subjects with newly diagnosed COPD. Thorax 2013;68(10):962-3. 


\section{CHAPTER 2}

134. Andersson $M$, Slinde F, Gronberg AM, et al. Physical activity level and its clinical correlates in chronic obstructive pulmonary disease: a cross-sectional study. Respir Res 2013;14:128.

135. Troosters T, Sciurba F, Battaglia S, et al. Physical inactivity in patients with COPD, a controlled multi-center pilot-study. Respir Med 2010;104(7):1005-11.

136. Pitta F, Troosters T, Spruit MA, et al. Characteristics of physical activities in daily life in chronic obstructive pulmonary disease. Am J Respir Crit Care Med 2005;171(9):972-7.

137. Ehsan $M$, Khan $R$, Wakefield $D$, et al. A longitudinal study evaluating the effect of exacerbations on physical activity in patients with chronic obstructive pulmonary disease. Ann Am Thorac Soc 2013;10(6):559-64.

138. Pitta F, Troosters T, Probst VS, et al. Physical activity and hospitalization for exacerbation of COPD. Chest 2006;129(3):536-44.

139. Waschki B, Spruit MA, Watz H, et al. Physical activity monitoring in COPD: compliance and associations with clinical characteristics in a multicenter study. Respir Med 2012;106(4):52230. [published Online First: 2011/11/29]

140. Ferguson GT, Fernandez E, Zamora MR, et al. Improved exercise performance following lung volume reduction surgery for emphysema. Am J Respir Crit Care Med 1998;157(4 Pt 1):1195203.

141. Hartman JE, Boezen HM, Heintzbergen S, et al. Daily physical activity after bronchoscopic lung volume reduction: a pilot study. Eur Respir J 2012;40(6):1566-7.

142. Lahaije AJ, van Helvoort HA, Dekhuijzen PN, et al. Physiologic limitations during daily life activities in COPD patients. Respir Med 2010;104(8):1152-9.

143. Vaes AW, Wouters EF, Franssen FM, et al.Task-related oxygen uptake during domesticactivities of daily life in patients with COPD and healthy elderly subjects. Chest 2011;140(4):970-9.

144. Calvert LD, Steiner MC, Morgan MD, et al. Plasma ammonia response to incremental cycling and walking tests in COPD. Respir Med 2010;104(5):675-81.

145. Criner $G J$, Belt $P$, Sternberg $A L$, et al. Effects of lung volume reduction surgery on gas exchange and breathing pattern during maximum exercise. Chest 2009;135(5):1268-79.

146. Budweiser S, Heinemann F, Meyer K, et al. Weight gain in cachectic COPD patients receiving noninvasive positive-pressure ventilation. Respir Care 2006;51(2):126-32. [published Online First: 2006/01/31]

147. Granger CL, McDonald CF, Irving L, et al. Low physical activity levels and functional decline in individuals with lung cancer. Lung Cancer 2014;83(2):292-9.

148. Hummler S, Thomas M, Hoffmann B, et al. Physical performance and psychosocial status in lung cancer patients: results from a pilot study. Oncology research and treatment 2014;37(12):36-41.

149. Hugli O, Frascarolo P, Schutz Y, et al. Diet-induced thermogenesis in chronic obstructive pulmonary disease. Am Rev Respir Dis 1993;148(6 Pt 1):1479-83.

150. Dore MF, Laaban JP, Orvoen-Frija E, et al. Role of the thermic effect of food in malnutrition of patients with chronic obstructive pulmonary disease. Am J Respir Crit Care Med 1997;155(5):1535-40.

151. Tang NL, Chung ML, Elia M, et al. Total daily energy expenditure in wasted chronic obstructive pulmonary disease patients. Eur J Clin Nutr 2002;56(4):282-7.

152. Schols A, Mostert R, Cobben N, et al. Transcutaneous oxygen saturation and carbon dioxide tension during meals in patients with chronic obstructive pulmonary disease. Chest 1991;100(5):1287-92.

153. Broekhuizen R, Wouters EF, Creutzberg EC, et al. Raised CRP levels mark metabolic and functional impairment in advanced COPD. Thorax 2006;61(1):17-22. [published Online First: 2005/08/02]

154. Staal-van den Brekel AJ, Schols AM, ten Velde GP, et al. Analysis of the energy balance in 
lung cancer patients. Cancer Res 1994;54(24):6430-3.

155. Langen RC, Haegens A, Vernooy JH, et al. NF-kappaB activation is required for the transition of pulmonary inflammation to muscle atrophy. Am J Respir Cell Mol Biol 2012;47(3):288-97.

156. Tkacova R, Ukropec J, Skyba P, et al. Increased adipose tissue expression of proinflammatory CD40, MKK4 and JNK in patients with very severe chronic obstructive pulmonary disease. Respiration 2011;81(5):386-93.

157. Fearon K, Strasser F, Anker SD, et al. Definition and classification of cancer cachexia: an international consensus. Lancet Oncol 2011;12(5):489-95. [published Online First: 2011/02/08]

158. Dahlman I, Mejhert N, Linder K, et al. Adipose tissue pathways involved in weight loss of cancer cachexia. British journal of cancer 2010;102(10):1541-8.

159. Schols AM, Creutzberg EC, Buurman WA, et al. Plasma leptin is related to proinflammatory status and dietary intake in patients with chronic obstructive pulmonary disease. Am J Respir Crit Care Med 1999;160(4):1220-6.

160. Brusik M, Ukropec J, Joppa P, et al. Circulatory and adipose tissue leptin and adiponectin in relationship to resting energy expenditure in patients with chronic obstructive pulmonary disease. Physiol Res 2012;61(5):469-80.

161. Kajimura S, Saito M. A new era in brown adipose tissue biology: molecular control of brown fat development and energy homeostasis. Annu Rev Physiol 2014;76:225-49. [published Online First: 2013/11/06]

162. Cinti S. Transdifferentiation properties of adipocytes in the adipose organ. Am J Physiol Endocrinol Metab 2009;297(5):E977-86.

163. Petruzzelli $M$, Schweiger $M$, Schreiber R, et al. A switch from white to brown fat increases energy expenditure in cancer-associated cachexia. Cell Metab 2014;20(3):433-47. [published Online First: 2014/07/22]

164. Vijgen GH, Bouvy ND, Teule GJ, et al. Brown adipose tissue in morbidly obese subjects. PLoS One 2011;6(2):e17247.

165. Yoneshiro T, Aita S, Matsushita M, et al. Age-related decrease in cold-activated brown adipose tissue and accumulation of body fat in healthy humans. Obesity (Silver Spring) 2011;19(9):1755-60.

166. Lee P, Greenfield JR, Ho KK, et al. A critical appraisal of the prevalence and metabolic significance of brown adipose tissue in adult humans. Am J Physiol Endocrinol Metab 2010;299(4):E601-6. [published Online First: 2010/07/08]

167. Cypess AM, Lehman S, Williams G, et al. Identification and importance of brown adipose tissue in adult humans. N Engl J Med 2009;360(15):1509-17. [published Online First: 2009/04/10]

168. Shellock FG, Riedinger MS, Fishbein MC. Brown adipose tissue in cancer patients: possible cause of cancer-induced cachexia. J Cancer Res Clin Oncol 1986;111(1):82-5. [published Online First: 1986/01/01]

169. Huang YC, Chen TB, Hsu CC, et al. The relationship between brown adipose tissue activity and neoplastic status: an (18)F-FDG PET/CT study in the tropics. Lipids Health Dis 2011;10:238. [published Online First: 2011/12/21]

170. Saure EW, Eagan TM, Jensen RL, et al. Explained variance for blood gases in a population with COPD. The clinical respiratory journal 2012;6(2):72-80.

171. Nakayama K, Frew IJ, Hagensen M, et al. Siah2 regulates stability of prolyl-hydroxylases, controls HIF1alpha abundance, and modulates physiological responses to hypoxia. Cell 2004;117(7):941-52.

172. van den Borst B, Schols AM, de Theije C, et al. Characterization of the inflammatory and metabolic profile of adipose tissue in a mouse model of chronic hypoxia. Journal of applied physiology 2013;114(11):1619-28. [published Online First: 2013/03/30] 


\section{CHAPTER 2}

173. Sun K, Wernstedt Asterholm I, Kusminski CM, et al. Dichotomous effects of VEGF-A on adipose tissue dysfunction. Proc Natl Acad Sci U S A 2012;109(15):5874-9.

174. Elias I, Franckhauser S, Ferre T, et al. Adipose tissue overexpression of vascular endothelial growth factor protects against diet-induced obesity and insulin resistance. Diabetes 2012;61(7):1801-13.

175. Franssen FM, Sauerwein HP, Ackermans MT, et al. Increased postabsorptive and exerciseinduced whole-body glucose production in patients with chronic obstructive pulmonary disease. Metabolism 2011;60(7):957-64.

176. Engelen MP, Schols AM, Does JD, et al. Altered glutamate metabolism is associated with reduced muscle glutathione levels in patients with emphysema. Am J Respir Crit Care Med 2000;161(1):98-103.

177. Engelen MP, Schols AM, Does JD, et al. Exercise-induced lactate increase in relation to muscle substrates in patients with chronic obstructive pulmonary disease. Am J Respir Crit Care Med 2000;162(5):1697-704.

178. Perez de Heredia F, Wood IS, Trayhurn P. Hypoxia stimulates lactate release and modulates monocarboxylate transporter (MCT1, MCT2, and MCT4) expression in human adipocytes. Pflugers Archiv : European journal of physiology 2010;459(3):509-18.

179. Carriere A, Jeanson Y, Berger-Muller S, et al. Browning of white adipose cells by intermediate metabolites: an adaptive mechanism to alleviate redox pressure. Diabetes 2014;63(10):325365.

180. Virtanen KA, Lidell ME, Orava J, et al. Functional brown adipose tissue in healthy adults. $\mathrm{N}$ Engl J Med 2009;360(15):1518-25.

181. Soderlund V, Larsson SA, Jacobsson H. Reduction of FDG uptake in brown adipose tissue in clinical patients by a single dose of propranolol. European journal of nuclear medicine and molecular imaging 2007;34(7):1018-22.

182. Hofford JM, Milakofsky L, Vogel WH, et al. The nutritional status in advanced emphysema associated with chronic bronchitis. A study of amino acid and catecholamine levels. Am Rev Respir Dis 1990;141(4 Pt 1):902-8.

183. Schiffelers SL, BlaakEE, Baarends EM, et al. beta-Adrenoceptor-mediated thermogenesis and lipolysis in patients with chronic obstructive pulmonary disease. Am J Physiol Endocrinol Metab 2001;280(2):E357-64.

184. Vosselman MJ, van der Lans AA, Brans B, et al. Systemic beta-adrenergic stimulation of thermogenesis is not accompanied by brown adipose tissue activity in humans. Diabetes 2012;61(12):3106-13.

185. Parysow O, Mollerach AM, Jager V, et al. Low-dose oral propranolol could reduce brown adipose tissue F-18 FDG uptake in patients undergoing PET scans. Clinical nuclear medicine 2007;32(5):351-7.

186. Lee H, Kim S, Lim Y, et al. Nutritional status and disease severity in patients with chronic obstructive pulmonary disease (COPD). Arch Gerontol Geriatr 2013;56(3):518-23.

187. Goris AH, Vermeeren MA, Wouters EF, et al. Energy balance in depleted ambulatory patients with chronic obstructive pulmonary disease: the effect of physical activity and oral nutritional supplementation. Br J Nutr 2003;89(5):725-31. [published Online First: 2003/05/02]

188. Schols AM, Soeters PB, Mostert R, et al. Energy balance in chronic obstructive pulmonary disease. Am Rev Respir Dis 1991;143(6):1248-52.

189. Creutzberg EC, Wouters EF, Vanderhoven-Augustin IM, et al. Disturbances in leptin metabolism are related to energy imbalance during acute exacerbations of chronic obstructive pulmonary disease. Am J Respir Crit Care Med 2000;162(4 Pt 1):1239-45. [published Online First: 2000/10/13]

190. Bruera E. ABC of palliative care. Anorexia, cachexia, and nutrition. BMJ 1997;315(7117):1219- 
22. [published Online First: 1997/12/11]

191. Pilhatsch $M$, Scheuing $H$, Kroemer $N$, et al. Nicotine administration in healthy non-smokers reduces appetite but does not alter plasma ghrelin. Human psychopharmacology 2014;29(4):384-7.

192. Eckerblad J, Todt K, Jakobsson P, et al. Symptom burden in stable COPD patients with moderate or severe airflow limitation. Heart \& lung:the journal of critical care 2014;43(4):3517.

193. Hilmarsen CW, Wilke S, Engan H, et al. Impact of symptoms of anxiety and depression on COPD Assessment Test scores. Eur Respir J 2014;43(3):898-900.

194. Gronberg AM, Slinde F, Engstrom CP, et al. Dietary problems in patients with severe chronic obstructive pulmonary disease. Journal of human nutrition and dietetics : the official journal of the British Dietetic Association 2005;18(6):445-52.

195. Sarna L, Lindsey AM, Dean $H$, et al. Weight change and lung cancer: relationships with symptom distress, functional status, and smoking. Research in nursing \& health 1994;17(5):371-9.

196. Iyer S, Roughley A, Rider A, et al. The symptom burden of non-small cell lung cancer in the USA: a real-world cross-sectional study. Support Care Cancer 2014;22(1):181-7.

197. Bentsen SB, Rustoen T, Miaskowski C. Prevalence and characteristics of pain in patients with chronic obstructive pulmonary disease compared to the Norwegian general population. J Pain 2011;12(5):539-45.

198. Roberts MH, Mapel DW, Hartry A, et al. Chronic pain and pain medication use in chronic obstructive pulmonary disease. A cross-sectional study. Ann Am Thorac Soc 2013;10(4):2908.

199. Ketwaroo GA, Cheng V, Lembo A. Opioid-induced bowel dysfunction. Curr Gastroenterol Rep 2013;15(9):344. [published Online First: 2013/08/01]

200. Portenoy RK, Ahmed E. Principles of opioid use in cancer pain. J Clin Oncol 2014;32(16):166270. [published Online First: 2014/05/07]

201. Jamshed N, Lee ZE, Olden KW. Diagnostic approach to chronic constipation in adults. Am Fam Physician 2011;84(3):299-306. [published Online First: 2011/08/17]

202. Tielemans MM, Jaspers Focks J, van Rossum LG, et al. Gastrointestinal symptoms are still prevalent and negatively impact health-related quality of life: a large cross-sectional population based study in The Netherlands. PLoS One 2013;8(7):e69876.

203. Veldhuizen MG, Albrecht J, Zelano C, et al. Identification of human gustatory cortex by activation likelihood estimation. Human brain mapping 2011;32(12):2256-66.

204. Oliveira-Maia AJ, Roberts CD, Simon SA, et al. Gustatory and reward brain circuits in the control of food intake. Advances and technical standards in neurosurgery 2011;36:31-59.

205. Norden J, Gronberg AM, Bosaeus I, et al. Nutrition impact symptoms and body composition in patients with COPD. Eur J Clin Nutr 2014 [published Online First: 2014/05/08]

206. Dewan NA, Bell CW, Moore J, et al. Smell and taste function in subjects with chronic obstructive pulmonary disease. Effect of long-term oxygen via nasal cannulas. Chest 1990;97(3):595-9.

207. Wardwell L, Chapman-Novakofski K, Brewer MS. Effects of age, gender and chronic obstructive pulmonary disease on taste acuity. International journal of food sciences and nutrition 2009;60 Suppl 6:84-97.

208. Janssen $P$, Vanden Berghe $P$, Verschueren $S$, et al. Review article: the role of gastric motility in the control of food intake. Aliment Pharmacol Ther 2011;33(8):880-94. [published Online First: 2011/02/24]

209. Karapanagiotou EM, Tsochatzis EA, Dilana KD, et al. The significance of leptin, adiponectin, and resistin serum levels in non-small cell lung cancer (NSCLC). Lung Cancer 2008;61 (3):391- 


\section{CHAPTER 2}

7.

210. Terzidis A, Sergentanis TN, Antonopoulos G, et al. Elevated serum leptin levels: a risk factor for non-small-cell lung cancer? Oncology 2009;76(1):19-25.

211. Itoh T, Nagaya $N$, Yoshikawa $M$, et al. Elevated plasma ghrelin level in underweight patients with chronic obstructive pulmonary disease. Am J Respir Crit Care Med 2004;170(8):879-82.

212. Karapanagiotou EM, Polyzos A, Dilana KD, et al. Increased serum levels of ghrelin at diagnosis mediate body weight loss in non-small cell lung cancer (NSCLC) patients. Lung Cancer 2009;66(3):393-8. [published Online First: 2009/03/14]

213. Sescousse G, Caldu X, Segura B, et al. Processing of primary and secondary rewards: a quantitative meta-analysis and review of human functional neuroimaging studies. Neurosci Biobehav Rev 2013;37(4):681-96. [published Online First: 2013/02/19]

214. Murdaugh DL, Cox JE, CookEW, 3rd, et al. fMRI reactivity to high-calorie food pictures predicts short- and long-term outcome in a weight-loss program. Neuroimage 2012;59(3):2709-21. [published Online First: 2012/02/15]

215. Patra SK, Arora S. Integrative role of neuropeptides and cytokines in cancer anorexiacachexia syndrome. Clin Chim Acta 2012;413(13-14):1025-34. [published Online First: 2012/01/19]

216. Luo N, Marcelin G, Liu SM, et al. Neuropeptide $Y$ and agouti-related peptide mediate complementary functions of hyperphagia and reduced energy expenditure in leptin receptor deficiency. Endocrinology 2011;152(3):883-9. [published Online First: 2011/02/03]

217. Briggs DI, Andrews ZB. Metabolic status regulates ghrelin function on energy homeostasis. Neuroendocrinology 2011;93(1):48-57. [published Online First: 2010/12/03]

218. Sanchez-Lara K, Arrieta O, Pasaye E, et al. Brain activity correlated with food preferences: a functional study comparing advanced non-small cell lung cancer patients with and without anorexia. Nutrition 2013;29(7-8):1013-9.

219. Sahebjami H, Vassallo CL. Influence of starvation on enzyme-induced emphysema. J Appl Physiol Respir Environ Exerc Physiol 1980;48(2):284-8.

220. Sahebjami H, MacGee J. Effects of starvation on lung mechanics and biochemistry in young and old rats. Journal of applied physiology 1985;58(3):778-84.

221. Sahebjami $H$, Domino $M$. Effects of starvation and refeeding on elastase-induced emphysema. Journal of applied physiology 1989;66(6):2611-6.

222. Bishai JM, Mitzner W. Effect of severe calorie restriction on the lung in two strains of mice. Am J Physiol Lung Cell Mol Physiol 2008;295(2):L356-62.

223. Coxson HO, Chan IH, Mayo JR, et al. Early emphysema in patients with anorexia nervosa. Am J Respir Crit Care Med 2004;170(7):748-52. [published Online First: 2004/07/17]

224. Thurlbeck WM. Diaphragm and body weight in emphysema. Thorax 1978;33(4):483-7. [published Online First: 1978/08/01]

225. Golgeli A, Ozesmi C, Suer C. Effect of acute starvation on rat diaphragm function. Jpn J Physiol 1994;44(6):743-7. [published Online First: 1994/01/01]

226. Efthimiou J, Fleming J, Gomes C, et al. The effect of supplementary oral nutrition in poorly nourished patients with chronic obstructive pulmonary disease. Am Rev Respir Dis 1988;137(5):1075-82.

227. Weekes CE, Emery PW, Elia M. Dietary counselling and food fortification in stable COPD: a randomised trial. Thorax 2009;64(4):326-31.

228. Engelen MP, Rutten EP, De Castro $C L$, et al. Casein protein results in higher prandial and exercise induced whole body protein anabolism than whey protein in chronic obstructive pulmonary disease. Metabolism 2012;61(9):1289-300. [published Online First: 2012/04/20]

229. Engelen MP, De Castro CL, Rutten EP, et al. Enhanced anabolic response to milk protein sip feeding in elderly subjects with COPD is associated with a reduced splanchnic extraction of 
multiple amino acids. Clin Nutr 2012;31(5):616-24. [published Online First: 2012/06/12]

230. van de Bool C, Mattijssen-Verdonschot C, van Melick PP, et al. Quality of dietary intake in relation to body composition in patients with chronic obstructive pulmonary disease eligible for pulmonary rehabilitation. Eur J Clin Nutr 2014;68(2):159-65. [published Online First: 2013/12/12]

231. Rutten EP, Lenaerts K, Buurman WA, et al. Disturbed intestinal integrity in patients with COPD: effects of activities of daily living. Chest 2014;145(2):245-52.

232. Engelen $M P$, Rutten $E P$, De Castro $C L$, et al. Altered interorgan response to feeding in patients with chronic obstructive pulmonary disease. Am J Clin Nutr 2005;82(2):366-72. [published Online First: 2005/08/10]

233. Pouw EM, Schols AM, Deutz NE, et al. Plasma and muscle amino acid levels in relation to resting energy expenditure and inflammation in stable chronic obstructive pulmonary disease. Am J Respir Crit Care Med 1998;158(3):797-801.

234. Kojima M, Hosoda H, Date Y, et al. Ghrelin is a growth-hormone-releasing acylated peptide from stomach. Nature 1999;402(6762):656-60. [published Online First: 1999/12/22]

235. Sun $Y$, Wang $P$, Zheng $H$, et al. Ghrelin stimulation of growth hormone release and appetite is mediated through the growth hormone secretagogue receptor. Proc Natl Acad Sci U S A 2004;101(13):4679-84. [published Online First: 2004/04/09]

236. Miki K, Maekura R, Nagaya N, et al. Effects of ghrelin treatment on exercise capacity in underweight COPD patients: a substudy of a multicenter, randomized, double-blind, placebo-controlled trial of ghrelin treatment. BMC Pulm Med 2013;13:37. [published Online First: 2013/06/14]

237. Garcia JM, Boccia RV, Graham CD, et al. Anamorelin for patients with cancer cachexia: an integrated analysis of two phase 2, randomised, placebo-controlled, double-blind trials. Lancet Oncol 2014

238. Currow DC, Abernethy AP. Anamorelin hydrochloride in the treatment of cancer anorexiacachexia syndrome. Future Oncol 2014;10(5):789-802. [published Online First: 2014/01/30]

239. Ziauddeen H, Farooqi IS, Fletcher PC. Obesity and the brain: how convincing is the addiction model? Nature reviews Neuroscience 2012;13(4):279-86.

240. Stice E, Yokum S, Burger KS, et al. Youth at risk for obesity show greater activation of striatal and somatosensory regions to food. J Neurosci 2011;31(12):4360-6. [published Online First: 2011/03/25]

241. Roefs A, Stapert D, Isabella LA, et al. Early associations with food in anorexia nervosa patients and obese people assessed in the affective priming paradigm. Eat Behav 2005;6(2):151-63. [published Online First: 2004/12/16]

242.Zipfel S, Wild B, Gross G, et al. Focal psychodynamic therapy, cognitive behaviour therapy, and optimised treatment as usual in outpatients with anorexia nervosa (ANTOP study): randomised controlled trial. Lancet 2014;383(9912):127-37.

243. Deckersbach T, Das SK, Urban LE, et al. Pilot randomized trial demonstrating reversal of obesity-related abnormalities in reward system responsivity to food cues with a behavioral intervention. Nutr Diabetes 2014;4:e129. [published Online First: 2014/09/02] 

INCREASED MYOGENIC AND PRØTEIN TURNOVER SIGNALING IN SKELETAL MUSCLE OF CHRONIC OBSTRUCTIXE PULMONARY DISEASE PATIENTS WITH SARCOPENIA

ANITA E.M. KNEPPERS RAMON C.J. LANGEN HARRY R. GOSKER LEX B. VERDIJK NANCA CEBRON LIPOVEC PIETER A. LEERMAKERS MARCO C.J.M. KELDERS CHIEL C. DE THEIJE DANIEL OMERSA MITJA LAINSCAK* \& ANNEMIE M.W.J. SCHOLS *these authors contributed equally 


\section{CHAPTER 3}

\section{ABSTRACT}

Background: Sarcopenia was recently recognized as an independent condition by an International Classification of Diseases, Tenth Revision, Clinical Modification code, and is a frequently observed comorbidity in chronic obstructive pulmonary disease (COPD). Muscle mass is primarily dictated by the balance between protein degradation and synthesis, but their relative contribution to sarcopenia is unclear.

Objective: We aimed to assess potential differential molecular regulation of protein degradation and synthesis, as well as myogenesis, in the skeletal muscle of COPD patients with and without sarcopenia.

Methods: Muscle biopsies were obtained from the vastus lateralis muscle. Patients with COPD were clustered based on sarcopenia defined by low appendicular skeletal muscle mass index (nonsarcopenic COPD, $n=53$; sarcopenic COPD, $n=39$ ), and compared with healthy nonsarcopenic controls $(n=13)$. The mRNA and protein expression of regulators and mediators of ubiquitin-proteasome system (UPS), autophagy-lysosome system (autophagy), and protein synthesis were analyzed. Furthermore, mRNA expression of myogenesis markers was assessed.

Results: UPS signaling was unaltered, whereas indices of UPS regulation (e.g. FOXO1 protein; p-FOXO3/FOXO3), autophagy signaling (e.g. LC3BII/l; p-ULK1(Ser757)/ULK1), and protein synthesis signaling (e.g. AKT1; p-GSK3B/GSK3B; p-4E-BP1/4E-BP1) were increased in COPD. These alterations were even more pronounced in COPD patients with sarcopenia (e.g. FOXO1 protein; p-FOXO1/FOXO1; LC3BII/l; p-ULK(Ser555); p-AKT1/AKT1; AKT1; p-4E-BP1). Furthermore, myogenic signaling (e.g. MYOG) was increased in COPD despite a concomitant increase of MSTN mRNA expression, with no difference between sarcopenic and nonsarcopenic COPD patients.

Conclusion: Together with elevated myogenic signaling, the increase in muscle protein turnover signaling in COPD, which is even more prominent in COPD patients with sarcopenia, reflects molecular alterations associated with muscle repair and remodeling. 


\section{INTRODUCTION}

Sarcopenia is defined as the loss of function in the presence of loss of muscle mass, and was recently recognized as an independent condition by an International Classification of Diseases, Tenth Revision, Clinical Modification code [1]. Apart from an age-associated decline in muscle mass, sarcopenia also encompasses the loss of muscle mass due to diseases such as chronic obstructive pulmonary disease (COPD) [2]. In COPD patients, sarcopenia is a frequently observed comorbidity, affecting exercise capacity [3, 4], quality of life [3], and survival [5-7]. Although muscle mass maintenance is considered to be primarily dictated by the balance between protein degradation and protein synthesis rates (i.e., protein turnover), their relative contribution to imbalanced protein turnover during muscle mass loss remains unclear.

To provide a first insight into the potential drivers of this imbalance between muscle protein synthesis and breakdown in COPD patients, several studies assessed the molecular regulation of skeletal muscle protein turnover in COPD, and nearly all point to both increased protein degradation signaling [8-14] and increased protein synthesis signaling $[10,11,15]$. These findings are in accordance with the reported increase in whole body protein turnover rate in COPD as assessed by stable isotopes [16]. It was previously suggested that increased protein synthesis signaling in COPD may serve to maintain muscle mass in the presence of an elevated protein degradation rate [11]. However, such a compensatory mechanism to prevent muscle atrophy appears insufficient, because the prevalence of sarcopenia in COPD patients is high (i.e., 12$33 \%$ ) [17-19]. Although there are some data available on protein turnover signaling in sarcopenic COPD patients $[8,9,11]$, these studies are either limited in sample size or focus solely on either protein degradation or protein synthesis signaling. Furthermore, the role of myogenesis in muscle mass maintenance is frequently overlooked or incompletely assessed.

A comprehensive analysis of myogenic and protein turnover regulation in COPD patients with and without sarcopenia would provide further insight into the underlying skeletal muscle pathology, potentially providing new targets for intervention. In the present study, we therefore aimed to assess potential differential regulation of protein degradation and synthesis, as well as myogenesis, through analysis of an extensive panel of molecular regulators and mediators of myogenic and protein turnover signaling in the skeletal muscle of COPD patients with and without sarcopenia compared with healthy controls. 


\section{CHAPTER 3}

\section{METHODS}

\section{Study design and participants}

The skeletal muscle molecular profiles of patients from 2 prospective cohort studies were analyzed. The study performed in Maastricht was registered at www.trialregister. $\mathrm{nl}$ as NTR1402, written informed consent was obtained from all participants, and the study was approved by the Maastricht University Medical Center+ (Maastricht, the Netherlands) ethical review board (08-2-059). The study design was previously published [20]. The study performed in Golnik was registered at www.clinicaltrials.gov as NCT02550808, written informed consent was obtained from all participants, and the study was approved by the Slovenian National Medical Ethics Committee (Ljubljana, Slovenia). The study design was previously published [21]. Only data from baseline (i.e., before pulmonary rehabilitation) measurements were used in the current study. All included patients were in a stable disease state, free from exacerbations in the 4 weeks before start of the study protocol.

Participants were excluded from the current analysis if the muscle biopsy or appendicular skeletal muscle mass index (ASMI) measurement was missing. Sarcopenia was solely defined according to cut-offs for ASMI $\left(<7.23 \mathrm{~kg} / \mathrm{m}^{2}\right.$ for men; $<5.76 \mathrm{~kg} /$ $\mathrm{m}^{2}$ for women) [22]; see Figure 1. One participant without COPD was classified as sarcopenic and was therefore excluded from the analyses, yielding a study population of 13 healthy controls and 92 COPD patients.

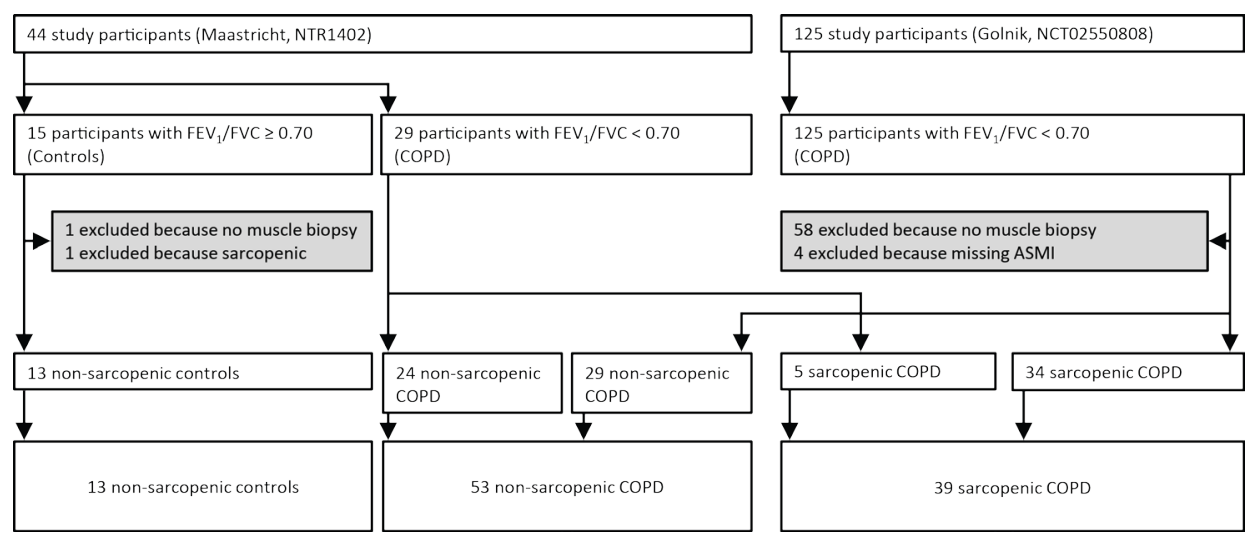

Figure 1.| Flow chart of participant selection and clustering. Abbreviations: ASMI, appendicular skeletal muscle mass index; FEV ${ }_{1}$, forced expiratory volume in 1 second; FVC, forced vital capacity; COPD, chronic obstructive pulmonary disease. Sarcopenia: ASMI $<7.23 \mathrm{~kg} / \mathrm{m}^{2}$ for men; $<5.67 \mathrm{~kg} / \mathrm{m}^{2}$ for women [22]. 


\section{Pulmonary and physical function}

Spirometry was used to obtain forced expiratory volume in 1 second $\left(\mathrm{FEV}_{1}\right)$, forced vital capacity (FVC) and their ratio ( $\mathrm{FEV}_{1} / \mathrm{FVC}$ ) according to the European Respiratory Society guidelines [23]. Patients were classified by disease severity based on Global Initiative for Chronic Obstructive Lung Disease (GOLD) stage [24]. Physical function was assessed by peak load, determined by an incremental load cycling test as previously described [25].

\section{Anthropometry and body composition}

Body Mass Index (BMI) was calculated as body mass $/$ height ${ }^{2}\left(\mathrm{~kg} / \mathrm{m}^{2}\right)$. Whole body DXA (Hologic QDR Series Explorer bone densitometer; Hologic Inc., MA, USA) was used to assess total and appendicular (i.e., arms and legs) fat-mass and fat-free mass. Fat-Free Mass Index (FFMI) was calculated as fat-free mass $/$ height $^{2}\left(\mathrm{~kg} / \mathrm{m}^{2}\right)$. ASMI was calculated as appendicular skeletal muscle mass $/$ height $^{2}\left(\mathrm{~kg} / \mathrm{m}^{2}\right)$.

\section{Muscle biopsy and analyses}

Biopsies were obtained from the $M$. vastus lateralis of the dominant leg by needle biopsy, at least 20 hours after the last exercise test. Muscle tissue was snap-frozen in liquid nitrogen, and stored at $-80^{\circ} \mathrm{C}$. Tissue processing and molecular analyses were performed collectively.

\section{RT-qPCR and Western Blotting}

Molecular analyses were performed in biopsies from all subjects. After removal of technical outliers, which varied between different analyses, the total sample size for protein and mRNA targets included 10-13 controls, 50-53 non-sarcopenic COPD patients, and 38-39 sarcopenic COPD patients.

Details on the procedures and the exact sample size per analyzed target are provided in the supplementary material.

\section{Statistics}

Differences between nonsarcopenic COPD patients, sarcopenic COPD patients and controls were tested by one-way ANOVA with Bonferroni post hoc comparisons in case of a significant group effect. Furthermore, differences between COPD patients and controls were tested using independent t-test. Relevant results of this comparison are presented in the figures. Analyses were performed using SPSS Statistics (version 22.0, IBM Corp., Armonk, NY, USA). A p-value $<0.05$ was considered statistically significant. 


\section{CHAPTER 3}

\section{RESULTS}

\section{Participant characteristics}

Based on gender-specific cut-offs for ASMI [22], 39 COPD patients (42\%) were sarcopenic, whereas 53 COPD patients (58\%) and all control subjects were nonsarcopenic. Patient characteristics are presented in Table 1.

Table 1. | General characteristics.

\begin{tabular}{|c|c|c|c|c|}
\hline & Control & $\begin{array}{c}\text { COPD, } \\
\text { no sarcopenia }\end{array}$ & $\begin{array}{c}\text { COPD, } \\
\text { sarcopenia }\end{array}$ & $\begin{array}{c}\text { Overall } \\
p \text {-value }\end{array}$ \\
\hline & $(n=13)$ & $(n=53)$ & $(n=39)$ & \\
\hline \multicolumn{5}{|l|}{ Demographics } \\
\hline Age, years & $64.5 \pm 5.4$ & $64.0 \pm 7.4$ & $66.4 \pm 8.4$ & NS \\
\hline Sex, \% male & $53.8 \%$ & $60.4 \%$ & $74.4 \%$ & NS \\
\hline \multicolumn{5}{|l|}{ Body composition } \\
\hline $\mathrm{BMI}, \mathrm{kg} / \mathrm{cm}^{2}$ & $24.8 \pm 3.4$ & $26.7 \pm 4.0$ & $22.9 \pm 2.8^{\ddagger \neq \ddagger}$ & $<0.001$ \\
\hline $\mathrm{ASMI}, \mathrm{kg} / \mathrm{cm}^{2}$ & $7.48 \pm 1.01$ & $7.42 \pm 1.01$ & $6.03 \pm 0.85^{* * * / \neq \neq \neq}$ & $<0.001$ \\
\hline female & $6.52 \pm 0.49$ & $6.43 \pm 0.61$ & $4.98 \pm 0.48^{* * * / \neq \neq \neq}$ & $<0.001$ \\
\hline male & $8.31 \pm 0.37$ & $8.06 \pm 0.64$ & $6.39 \pm 0.61^{* * * / \neq \neq \neq}$ & $<0.001$ \\
\hline $\mathrm{FFMI}, \mathrm{kg} / \mathrm{cm}^{2}$ & $17.9 \pm 1.9$ & $18.0 \pm 2.5$ & $15.9 \pm 2.4^{* / \neq \neq \neq}$ & $<0.001$ \\
\hline female & $16.3 \pm 1.4$ & $15.9 \pm 1.3$ & $13.1 \pm 1.1^{* * * / \neq \neq \neq}$ & $<0.001$ \\
\hline male & $19.2 \pm 1.0$ & $19.5 \pm 2.0$ & $16.8 \pm 1.9^{* / \neq \ddagger \neq}$ & $<0.001$ \\
\hline \multicolumn{5}{|l|}{ Physical function } \\
\hline Peak load, Watt & $190.3 \pm 62.8$ & $73.4 \pm 24.4^{* * * *}$ & $58.1 \pm 24.9^{* * * / \dagger}$ & $<0.001$ \\
\hline female & $136.0 \pm 6.3$ & $65.5 \pm 19.2^{* * *}$ & $47.0 \pm 19.5^{* * * / \neq}$ & $<0.001$ \\
\hline male & $236.9 \pm 48.9$ & $78.1 \pm 26.1^{* * *}$ & $62.3 \pm 25.7^{* * * *}$ & $<0.001$ \\
\hline \multicolumn{5}{|l|}{ Lung function } \\
\hline $\mathrm{FEV}_{1}, \%$ predicted & $113.5 \pm 12.9$ & $48.6 \pm 18.3^{* * *}$ & $36.4 \pm 12.9^{* * * / \neq \neq}$ & $<0.001$ \\
\hline $\mathrm{FEV}_{1} / \mathrm{FVC}, \%$ & $73.1 \pm 4.9$ & $39.5 \pm 12.2^{* * *}$ & $34.5 \pm 9.8^{* * * / \dagger}$ & $<0.001$ \\
\hline GOLD, $\%$ & & & & $<0.05$ \\
\hline 1 & & $6 \%$ & $0 \%$ & \\
\hline 2 & & $32 \%$ & $13 \%$ & \\
\hline 3 & & $45 \%$ & $56 \%$ & \\
\hline 4 & & $17 \%$ & $31 \%$ & \\
\hline
\end{tabular}

Abbreviations: BMI, body mass index; ASMI, appendicular skeletal muscle mass index; FFMI, fat-free mass index; FEV ${ }_{1}$, forced expiratory volume in 1 second; FVC, forced vital capacity; GOLD, Global Initiative for Chronic Obstructive Lung Disease. Data presented as mean \pm SD. Significant post-hoc comparisons after Bonferroni correction: ${ }^{*} p<0.05,{ }^{* *} p<0.01,{ }^{* * *} p<0.001$, compared with control group; $t p<0.10, \neq p<0.05, \neq \neq p<0.01, \neq \neq \neq p<0.001$, compared to nonsarcopenic COPD group.

Although the sarcopenic COPD group contained slightly more men, groups did not differ significantly in sex distribution and age. BMI was lower in sarcopenic than in nonsarcopenic COPD patients, but controls did not differ from either COPD sub-group. Besides ASMI, FFMI was lower in sarcopenic COPD patients than in nonsarcopenic COPD patients and controls. Furthermore, physical function was decreased in COPD patients compared with controls, and tended to be even further decreased in sarcopenic COPD patients compared with nonsarcopenic COPD patients. $\mathrm{FEV}_{1} / \mathrm{FVC}$ ratio and $\mathrm{FEV}_{1}(\%$ 
predicted) were lower in COPD patients than in controls, and $\mathrm{FEV}_{1}$ (\% predicted) was even lower in sarcopenic than in nonsarcopenic COPD patients. Correspondingly, nonsarcopenic COPD patients most often had moderate (32\%) to severe (45\%) COPD, whereas sarcopenic COPD patients most often had severe $(56 \%)$ to very severe $(31 \%)$ COPD based on GOLD stage. Together, this shows that lung function is more impaired in sarcopenic than in nonsarcopenic COPD patients.

\section{Protein degradation signaling}

To assess potential differential regulation of skeletal muscle protein degradation signaling in COPD and sarcopenic COPD, markers of the 2 major proteolytic pathways were measured; that is, the ubiquitin-proteasome system (UPS) and the autophagylysosome system (autophagy), which are in part regulated by forkhead box O (FOXO) transcription factors, including FOXO1 and FOXO3.

\section{Progressively increased FOXO signaling in COPD and sarcopenic COPD}

FOXO1 relative phosphorylation was unaltered in the skeletal muscle of nonsarcopenic COPD patients, but tended to be lower in sarcopenic COPD patients than in controls (Figure 2A). Remarkably, FOXO1 protein levels were higher in both nonsarcopenic and sarcopenic COPD patients than in controls, and were even further elevated in sarcopenic COPD patients compared with nonsarcopenic COPD patients (Figure 2C). This was paralleled by changes in FOXO1 mRNA levels, which appeared higher in both nonsarcopenic and sarcopenic COPD patients, but reached significance only in sarcopenic COPD patients compared with controls (Figure 2D). Absolute FOXO1 inhibitory phosphorylation was concomitantly increased (Figure 2B), which may reflect an adaptation to compensate for the elevated FOXO1 expression. No group differences were found in $\mathrm{FOXO} 3$ mRNA and protein expression or absolute phosphorylation levels (Figure 2F, G, H); however, relative FOXO3 phosphorylation was lower in both nonsarcopenic and sarcopenic COPD patients than in controls (Figure 2E).

\section{Unaltered ubiquitin proteasome system signaling}

In contrast to the apparent increase in FOXO activity, mRNA expression levels of the E3 ligases TRIM63 (tripartite motif containing 63 (MURF1)) and FBXO30 (F-box protein 30 (MUSA1)) were unaltered in the skeletal muscle of nonsarcopenic and sarcopenic COPD patients (Figure 3A, B). However, FBXO21 (F-ox protein 21 (SMART)) mRNA expression was higher in sarcopenic COPD patients than in controls (Figure $3 C$ ), whereas FBXO32/MAFBX (F-box protein 32 (ATROGIN1)) mRNA expression tended to be lower in sarcopenic COPD patients than in nonsarcopenic COPD patients (Figure 3D). 


\section{CHAPTER 3}

Control

COPD, no sarcopenia

COPD, sarcopenia

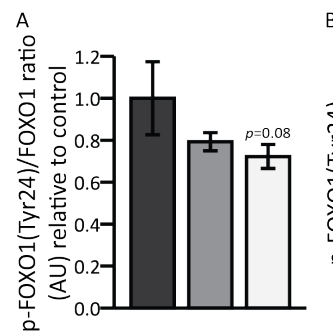

B

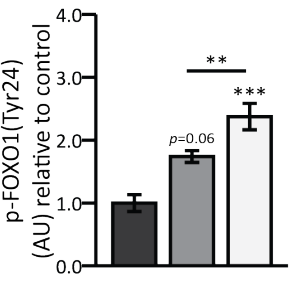

C

D

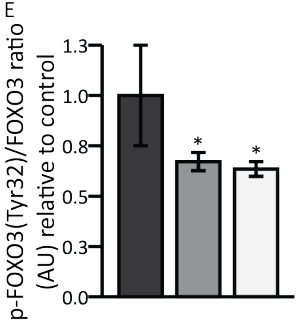

$\mathrm{F}$
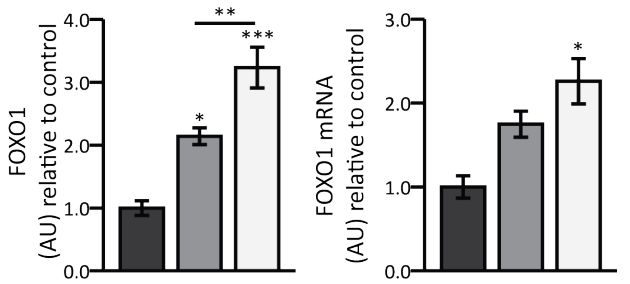

G

$\mathrm{H}$
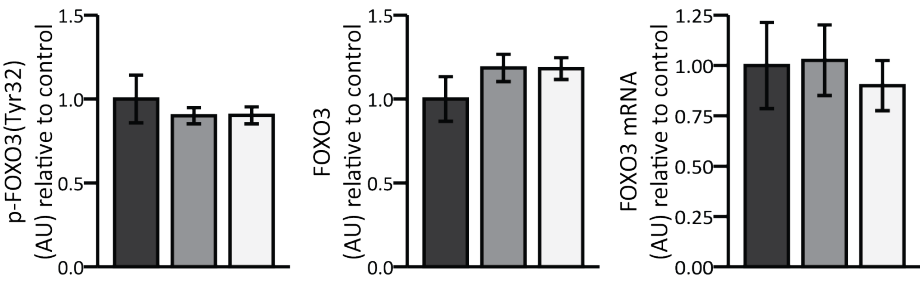

Figure 2. |FOXO regulation in COPD and sarcopenic COPD. A) Relative FOXO1 phosphorylation at Tyr24, B) Absolute FOXO1 phosphorylation at Tyr24, C) FOXO1 protein expression, D) FOXO1 mRNA expression, E) Relative FOXO3 phosphorylation at Tyr32, F) Absolute FOXO3 phosphorylation at Tyr32, G) FOXO3 protein expression, H) FOXO3 mRNA expression. Abbreviations: FOXO1, forkhead box 01; FOXO3, forkhead box O3; Tyr, tyrosine; Ser, Serine. Values are means $\pm S E M .{ }^{*} p<0.05$, ${ }^{* *} p<0.01,{ }^{* * *} p<0.001$, compared to control group or between indicated groups.

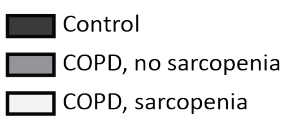

A

B

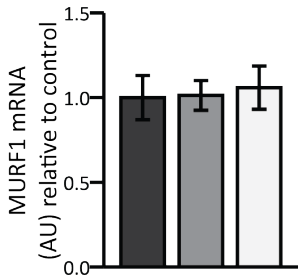

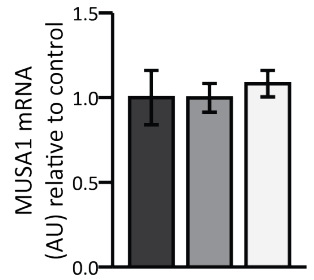

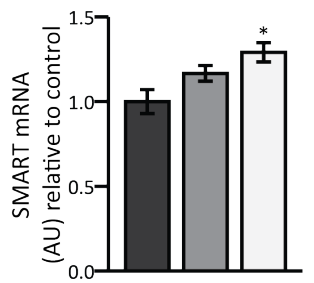

D

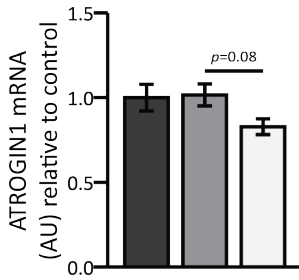

Figure 3. I mRNA expression of UPS markers in COPD and sarcopenic COPD. A) MURF1 mRNA expression, B) MUSA1 mRNA expression, C) SMART mRNA expression, D) ATROGIN1 mRNA expression. Abbreviations: MURF1, tripartite motif containing 63; MUSA1, F-box protein 30; SMART, F-box protein 21; ATROGIN1, F-box protein 32. Values are means \pm SEM. * $p<0.05$, compared to control group or between indicated groups. 


\section{Progressively increased autophagy signaling in COPD and sarcopenic COPD}

Among the FOXO target genes are the autophagy-related genes MAP1LC3B (microtubule associated protein 1 light chain 3 beta (LC3B)), SQSTM1 (sequestosome 1 (P62)), and BECN1 (Beclin 1) [26, 27]. In line with the unaltered MURF1 and MUSA1 mRNA levels, LC3B and P62 mRNA expression remained unaltered (Figure 4D, F). BECN1 mRNA expression, however, was higher in COPD patients than in controls (Figure 4H). Interestingly, LC3BI protein expression appeared higher in both nonsarcopenic and sarcopenic COPD patients but reached significance only in sarcopenic COPD patients compared with controls, whereas LC3BII levels were unaltered (Figure 4B, C). The resulting lower LC3BII/I ratio in sarcopenic COPD patients than in controls (Figure 4A) implies a change in autophagic flux, although no change in $\mathrm{P} 62$ protein expression was observed (Figure 4E). In line with the altered LC3BII/I ratio, BECN1 protein expression seemed to be higher in sarcopenic COPD patients (Figure 4G), but this did not reach statistical significance.

An important juncture in the initiation of autophagy is activation of unc-51 like autophagy activating kinase 1 (ULK1), which is regulated through phosphorylation by the upstream kinases; mechanistic target of rapamycin (MTOR) complex 1 (MTORC1) and AMPK. ULK1 relative inhibitory phosphorylation (Ser757) was lower in both nonsarcopenic and sarcopenic COPD patients than in controls (Figure 4I), whereas relative ULK1 activating phosphorylation (Ser555) was unaltered (Figure 4K). Furthermore, absolute ULK1 Ser757 phosphorylation, and ULK1 protein and mRNA expression were unaltered (Figure 4J, M, N), whereas absolute ULK1 Ser555 phosphorylation tended to be higher in sarcopenic COPD patients than in controls and nonsarcopenic COPD patients (Figure 4L).

\section{Progressively increased AKT/MTOR signaling in COPD and sarcopenic COPD}

Coordination of protein degradation and protein synthesis signaling occurs via the PI3K/PKB (serine/threonine kinase 1 (AKT1))/MTOR pathway. The relative phosphorylation of AKT1 was lower in the skeletal muscle of both nonsarcopenic and sarcopenic COPD patients than in controls, which tended to be more pronounced in sarcopenic COPD patients compared with nonsarcopenic COPD patients (Figure 5A). This reduced relative AKT1 phosphorylation did not result from a change in absolute AKT1 phosphorylation levels (Figure 5B) but from a higher AKT1 protein expression in nonsarcopenic and sarcopenic COPD patients than in controls, and in sarcopenic COPD patients compared with nonsarcopenic COPD patients (Figure 5C). Despite the change in AKT1 regulation, MTOR phosphorylation (Figure 5D, E) and expression (Figure 5F) 


\section{CHAPTER 3}

were unaltered. However, relative and absolute phosphorylation of the downstream AKT1 target glycogen synthase kinase 3 beta (GSK3B) were higher in sarcopenic COPD patients than in controls (Figure 5G, H, I), suggesting increased AKT1 activity.

A

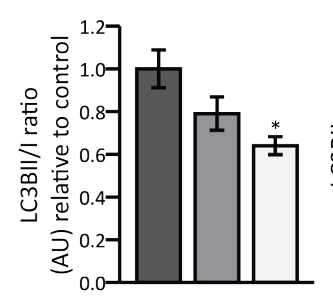

B

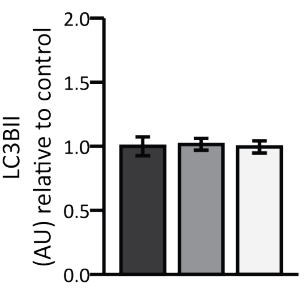

E
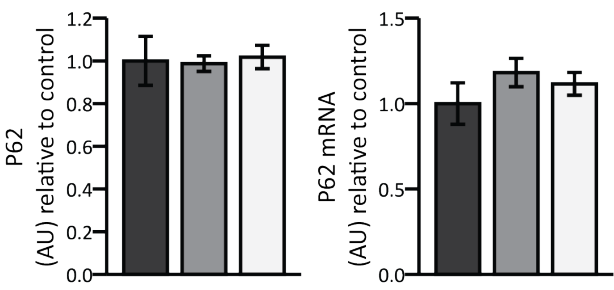

J
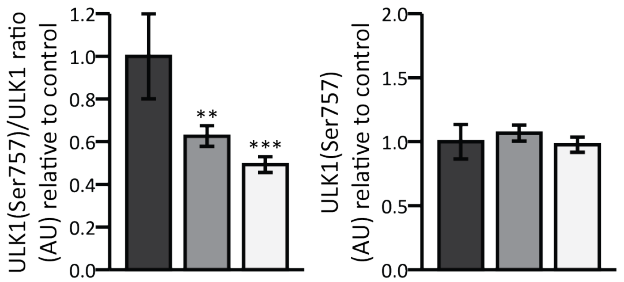

c

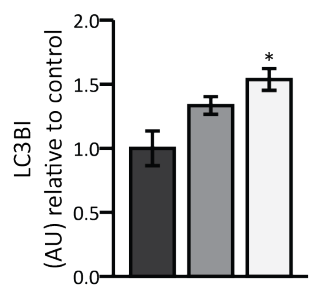

D

G

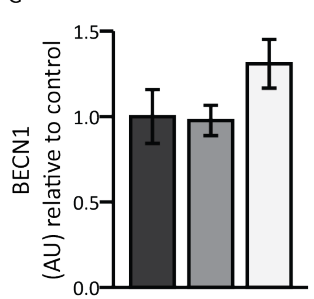

H

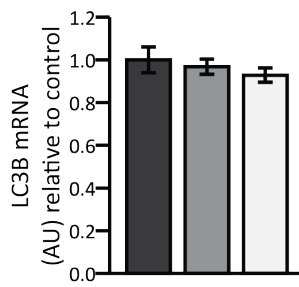

H

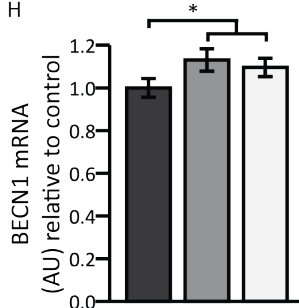

K

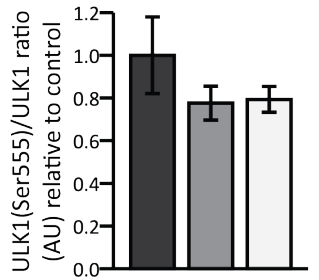

$\mathrm{N}$

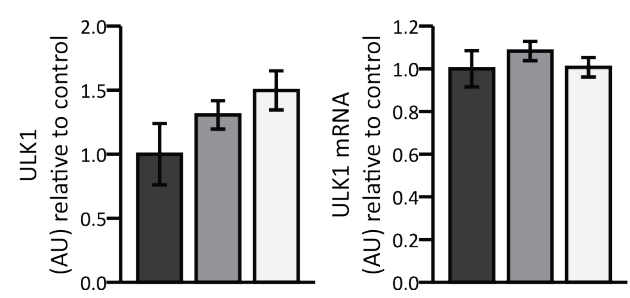

M

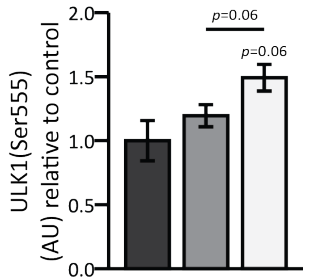

Figure 4. | Autophagic flux and autophagy initiation markers in COPD and sarcopenic COPD. A) $L C 3 B I I / I$ protein ratio, B) LC3BII protein expression, C) LC3BI protein expression, D) LC3B mRNA expression, E) P62 protein expression, F) P62 mRNA expression, G) BECN1 protein expression, H) BECN1 mRNA expression, I) Relative ULK1 phosphorylation at Ser757, J) Absolute ULK1 phosphorylation at Ser757, K) Relative ULK1 phosphorylation at Ser555, L) Absolute ULK1 phosphorylation at Ser555, M) ULK1 protein expression, N) ULK1 mRNA expression. Abbreviations: LC3B, microtubule associated protein 1 light chain 3 beta; P62, sequestosome 1; BECN1, Beclin1; ULK1, unc-51 like autophagy activating kinase 1; Tyr, tyrosine; Ser, Serine. Values are means \pm SEM. ${ }^{*} p<0.05,{ }^{* *} p<0.01,{ }^{* * *} p<0.001$, compared to control group or between indicated groups. 

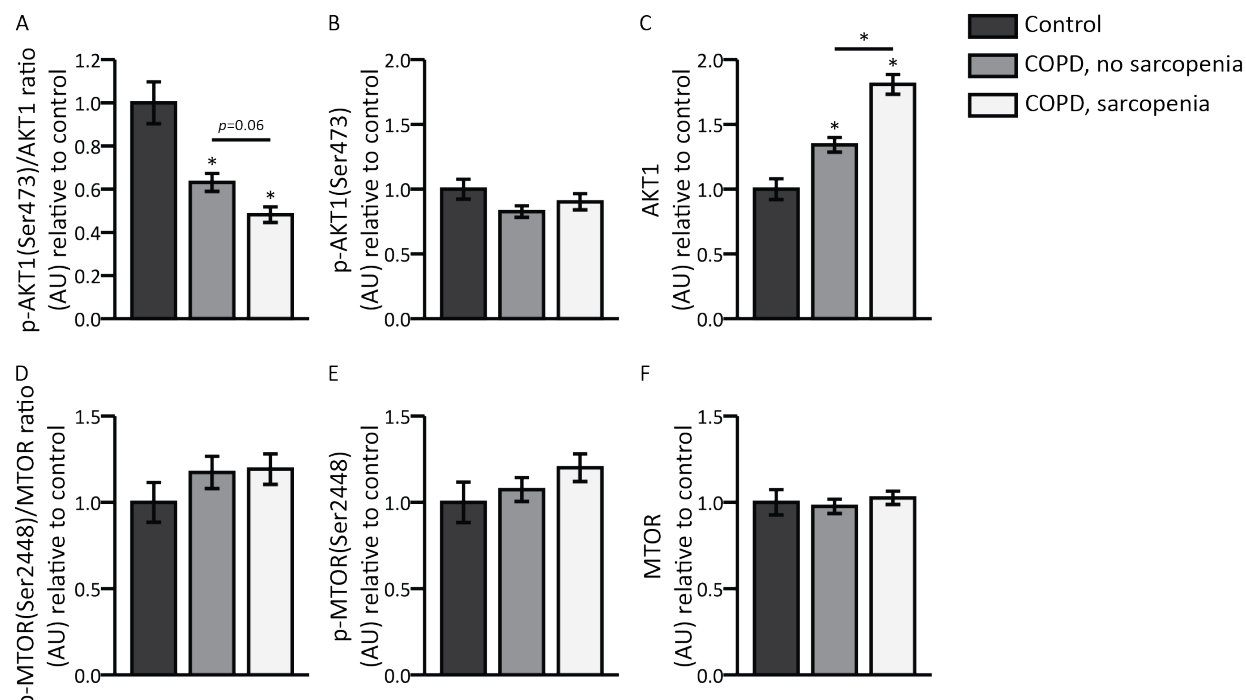

E

$\mathrm{F}$
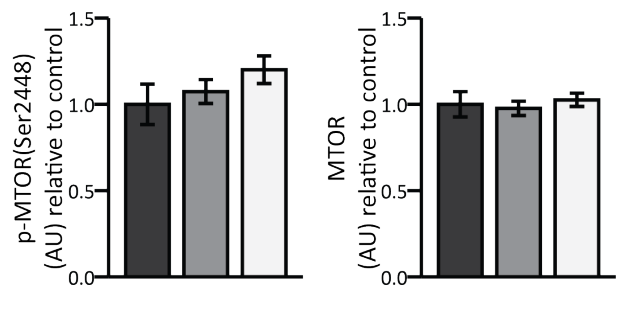

G

H
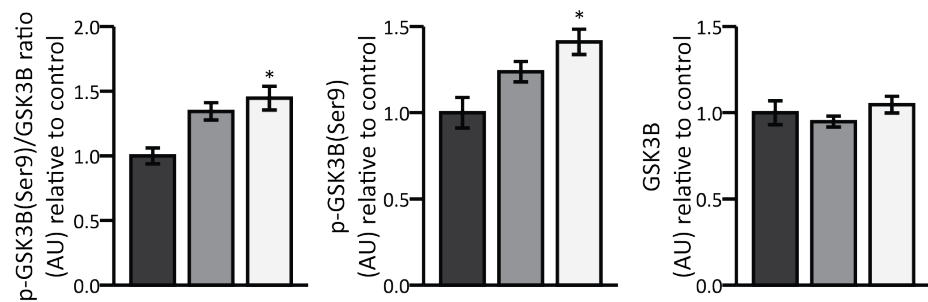

Figure 5. AKT/MTOR signaling in COPD and sarcopenic COPD. A) Relative AKT phosphorylation at Ser473, B) Absolute AKT phosphorylation at Ser473, C) AKT protein expression, D) Relative MTOR phosphorylation at Ser2448, E) Absolute MTOR phosphorylation at Ser2448, F) MTOR protein expression, G) Relative GSK3B phosphorylation at Ser9, H) Absolute GSK3B phosphorylation at Ser9, 1) GSK3B protein expression. Abbreviations: AKT1, AKT serine/threonine kinase 1; MTOR, mechanistic target of rapamycin; GSK3B, glycogen synthase kinase 3 beta; Tyr, tyrosine; Ser, Serine. Values are means $\pm S E M .{ }^{*} p<0.05$, compared to control group or between indicated groups.

\section{Progressively increased protein synthesis signaling in COPD and sarcopenic COPD}

Although the MTORC1 downstream effector ribosomal protein S6 (RPS6) displayed unaltered relative phosphorylation (Figure $6 \mathrm{~A}$ ), both RPS6 protein expression and absolute phosphorylation were higher in COPD patients than in controls (Figure 6B, C). Furthermore, both relative and absolute phosphorylation of the translation repressor EIF4EBP1 (eukaryotic translation initiation factor 4E binding protein 1 (4E-BP1)) were higher in sarcopenic COPD patients than in controls (Figure 6D, E, F). 

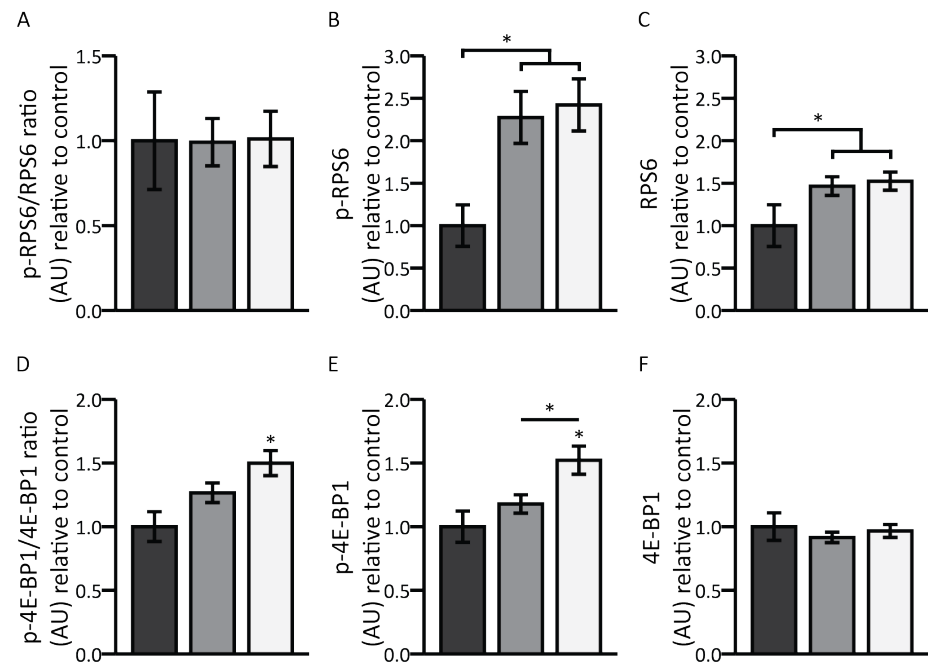

E

$\mathrm{F}$
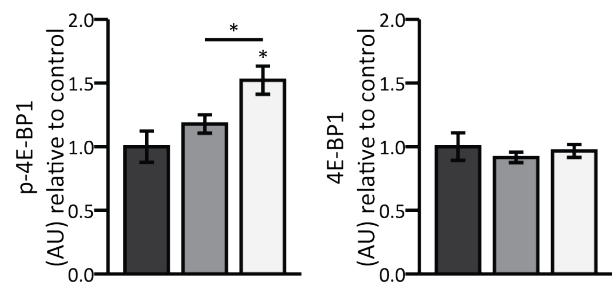

Figure 6.|Protein synthesis signaling in COPD and sarcopenic COPD. A) Relative RPS6 phosphorylation, B) Absolute RPS6 phosphorylation, C) RPS6 protein expression, D) Relative 4E-BP1 phosphorylation, E) Absolute $4 E-B P 1$ phosphorylation, F) 4E-BP1 protein expression. Abbreviations: RPS6, ribosomal protein S6; $4 E-B P 1$, eukaryotic translation initiation factor $4 E$ binding protein 1; $T y r$, tyrosine; Ser, Serine. Values are means \pm SEM. ${ }^{*} p<0.05$, compared to control group or between indicated groups.

\section{Increased myogenic signaling in COPD}

Increased protein degradation and protein synthesis signaling in COPD and sarcopenic COPD suggest an increase in protein turnover. A similar pattern of molecular alterations has been observed during muscle remodeling after exercise [28]. We therefore asked if myogenesis, a process that is also implicated in muscle repair and remodeling, is increased in COPD and sarcopenic COPD patients as well. To this end, markers of satellite cell function were evaluated. The mRNA expression levels of the satellite cell marker paired box 7 (PAX7) and the proliferation markers proliferation cell nuclear antigen (PCNA) and cyclin D1 (CCND1) were unaltered in nonsarcopenic and sarcopenic COPD patients (Figure 7A, B, C). Furthermore, the mRNA expression of myogenic factor 5 (MYF5) was unaltered (Figure 7D). In contrast, myogenic differentiation 1 (MYOD1) and myogenin (MYOG) mRNA expression was higher in COPD patients than in controls (Figure 7E, F), and CDH15 (cadherin 15 (M-cadherin)) mRNA expression showed a similar trend (Figure 7G). This implicates a sarcopenia-independent increase in the mRNA expression of muscle differentiation markers in COPD. In discordance with these findings which suggested increased myogenesis, the mRNA expression of the myogenesis repressor MSTN was higher in the skeletal muscle of both nonsarcopenic and sarcopenic COPD patients than in controls (Figure $7 \mathrm{H}$ ). 
A

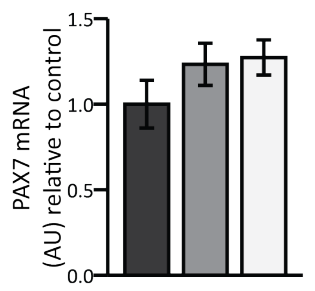

$\mathrm{E}$

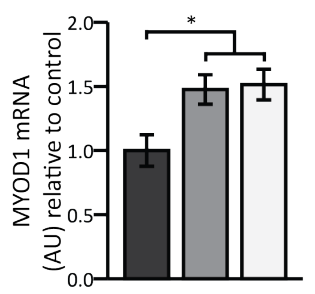

B

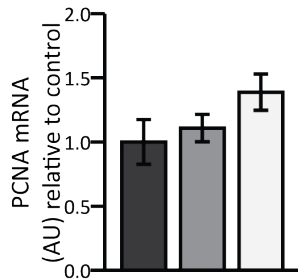

$\mathrm{F}$

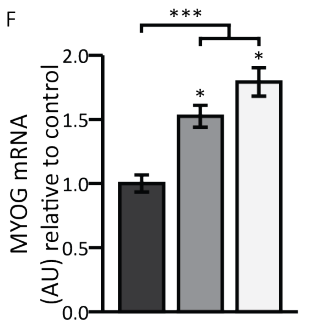

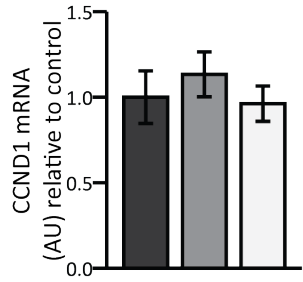

G

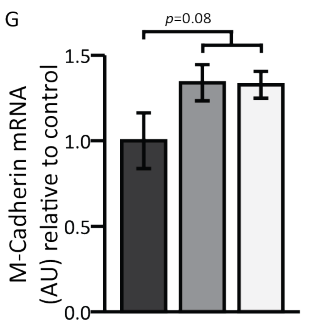

D
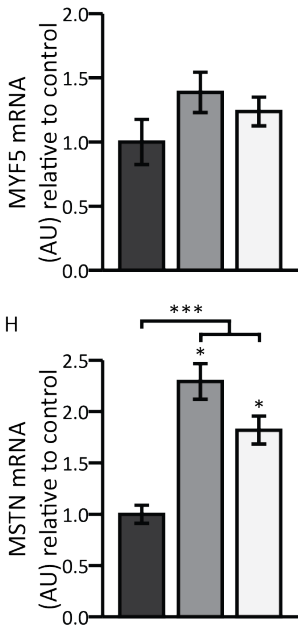

Figure 7. I mRNA expression of myogenic markers in COPD and sarcopenic COPD. A) PAX7 MRNA expression, B) PCNA mRNA expression, C) CCND1 mRNA expression, D) MYF5 mRNA expression, E) MYOD1 mRNA expression, F) MYOG mRNA expression, G) M-cadherin mRNA expression, H) MSTN mRNA expression. Abbreviations: PAX7, paired box 7; PCNA, proliferating cell nuclear antigen; CCND1, cyclin D1; MYF5, myogenic factor 5; MYOD1, myogenic differentiation 1; MYOG, myogenin; $M$-cadherin, cadherin 15; MSTN, myostatin. Values are means \pm SEM. ${ }^{*} p<0.05$, compared to control group.

\section{DISCUSSION}

By assessing a comprehensive panel of molecular regulators of muscle protein turnover, we demonstrate that both protein degradation and synthesis signaling are increased in the skeletal muscles of COPD patients, and extend previous findings by showing that these alterations are even more prominent in sarcopenic COPD patients. Furthermore, we show that myogenic differentiation signaling is increased in COPD, which is maintained, but not aggravated in sarcopenic COPD.

Several studies have addressed protein degradation and/or protein synthesis signaling in COPD or sarcopenic COPD $[8-14,29,30]$. However, due to differences in characteristics of the control groups, differences in disease severity of the COPD populations, and differences in body composition phenotyping, these studies cannot be directly compared or combined to extrapolate a conclusion on protein turnover regulation in COPD-related sarcopenia. In the present study, we therefore measured an extensive panel of molecular markers in skeletal muscle biopsies of a large, well- 


\section{CHAPTER 3}

phenotyped COPD cohort to assess potential differential regulation of protein degradation and synthesis in the skeletal muscle of COPD patients with and without sarcopenia, and hint toward its potential implications for the level of protein turnover. Although we recognize that muscle protein degradation and synthesis rates were not measured in this study, given that this is a general limitation of many studies in humans, coordination between signaling and rates of turnover can be extrapolated from previous studies [31].

Previous studies suggested increased FOXO signaling in the skeletal muscle of COPD patients and sarcopenic COPD patients $[11,29]$. Correspondingly, we find lower relative FOXO1 and FOXO3 inhibitory phosphorylation in COPD patients, implicating increased FOXO transcriptional activity. In line, the mRNA expression of the FOXO target SMART is higher in sarcopenic COPD patients than in controls. However, although previous reports showed increased ATROGIN1 [8,9, 13] and MURF1 [11,29] expression in COPD and sarcopenic COPD, the present study shows no increase in the mRNA expression of ATROGIN1, MURF1, or MUSA1. In agreement with the study of Natanek et al. [30], ATROGIN1 mRNA expression even tends to be lower in sarcopenic COPD patients than in nonsarcopenic COPD patients. Although the current data seem in disagreement with some of the previous literature, we and others have suggested that the increased expression of E3 ligases might be a feature of acute catabolic events rather than chronic muscle wasting [30,32,33], the latter of which was studied in this cohort. We speculate that a recurring rise in UPS activity during repeated disease exacerbations results in an increased capacity for UPS induction, rendering the skeletal muscle more susceptible to catabolic triggers [34, 35]. The higher FOXO1 protein and mRNA expression levels in muscle tissue of COPD patients and sarcopenic COPD patients may reflect such an increase in UPS inductive capacity. In this regard, the elevated absolute FOXO1 phosphorylation level may serve as a compensatory inhibition of (excess) protein degradation signaling in the stable disease phase.

In line with unaltered E3 ligase expression, the mRNA expression of autophagyrelated FOXO target genes, $\mathrm{LC} 3 \mathrm{~B}$ and $\mathrm{P} 62$, is unchanged in COPD and sarcopenic COPD patients. Nonetheless, the higher BECN1 mRNA expression level in COPD patients than in controls, and the apparent elevation in BECN1 protein expression in sarcopenic compared with nonsarcopenic COPD patients hint toward increased autophagy in COPD and sarcopenic COPD. It was previously shown that the number of autophagosomes is higher in the skeletal muscle of COPD patients and muscle-wasted COPD patients than in controls [14, 29]. Although autophagic flux cannot be measured in vivo in humans, the lower LC3BII/I ratio in sarcopenic COPD patients compared with 
controls indicates that the level of autophagic flux is altered. The relative inhibitory phosphorylation (Ser757) of ULK1, an important juncture in autophagy initiation, is lower in COPD patients than in controls. Furthermore, the absolute Ser555 phosphorylation level tends to be higher in sarcopenic COPD patients than in controls or nonsarcopenic COPD patients. Together, this suggests that in the skeletal muscle of COPD patients, and even more prominent in sarcopenic COPD patients, autophagy is increased.

Higher absolute FOXO1 phosphorylation levels in COPD and sarcopenic COPD patients can result from increased AKT1 activity. However, several previous studies suggested a decreased AKT1 activity in COPD and sarcopenic COPD based on the relative AKT1 phosphorylation $[11,14]$. In line with this, we find lower relative AKT1 phosphorylation in nonsarcopenic COPD patients than in controls, and a trend toward a further decrease in sarcopenic COPD patients compared with nonsarcopenic COPD patients. Notably, the lower AKT1 relative phosphorylation is not reflective of altered absolute AKT1 phosphorylation, but rather of higher AKT1 protein expression. Moreover, phosphorylation levels of other downstream targets of AKT1 (i.e., GSK3B, RPS6, 4EBP1), suggest increased AKT1 activity. This stresses the need to not merely predict the enzymatic activity of phosphoproteins based on their relative phosphorylation state, but to also consider alterations in total protein abundance. Indeed, although the relative RPS6 phosphorylation remains unaltered, both the expression and activating phosphorylation level of RPS6 are higher in COPD patients than in controls, which is suggestive of increased mRNA translation capacity. Furthermore, the absolute inhibitory phosphorylation level of the negative regulators of translation initiation, GSK3B and 4E-BP1, is higher in sarcopenic COPD patients than in controls, implicating increased mRNA translation initiation. Together, these results show that the stimulatory signaling and capacity for protein synthesis is increased in the skeletal muscle of COPD patients in a stable disease state, particularly in those with sarcopenia.

Alterations in protein turnover are not necessarily accompanied by changes in muscle mass, and may serve several physiological processes, including muscle remodeling [28]. For example, recovery from muscle injury caused by eccentric exercise is accompanied by an increase in protein synthesis signaling [36]. Furthermore, a recent study showed an induction of autophagy during recovery from muscle injury and suggested a role for autophagy in muscle regeneration [37]. In the present study, muscle biopsies were taken at a basal state. Therefore, the increased autophagy and protein synthesis signaling suggest ongoing skeletal muscle remodeling in COPD and sarcopenic COPD patients, which may relate to muscle phenotypic alterations or damage repair. Indeed, previous studies showed damage of sarcomeres in the skeletal muscle of cachectic 


\section{CHAPTER 3}

COPD patients [29]. Furthermore, recovery from muscle damaging eccentric exercise is accompanied by an increase in skeletal muscle satellite cell content [38], which is indicative of myogenesis. Thèriault et al. reported unaltered satellite cell numbers in COPD patients with and without muscle wasting, whereas the number of central nuclei was higher in COPD patients with preserved muscle mass [39]. Based on myogenic regulatory factor (MRF) protein expression, they suggested that satellite cells in COPD are under a sustained proliferative state. The current study, however, shows no change in the mRNA expression of PAX7, PCNA, and CCND1, suggesting no alteration in satellite cell numbers or proliferation in muscle of COPD patients. Furthermore, MYF5 mRNA expression is unaltered, whereas the mRNA expression levels of M-cadherin and the MRFs, MYOD1 and MYOG, are higher in COPD patients than in controls. Together, this points toward increased myogenic differentiation signaling in COPD, which is maintained, but not aggravated, in sarcopenic COPD.

Strikingly, but in line with previous research [13], MSTN mRNA expression is higher in the skeletal muscle of COPD patients than in controls. MSTN is commonly known as a negative regulator of protein synthesis and myogenesis [40]. However, when considering the seemingly contradictory increase in expression of both MSTN and MRFs in COPD, it should be taken into account that the effect of MSTN is context dependent $[41,42]$. In vitro data showed that MSTN negatively affects myoblast proliferation and myogenic differentiation [43, 44], and in vivo MSTN deficiency elevates satellite cell numbers due to increased self-renewal [41]. As such, it seems obvious that proliferation is inhibited by MSTN. However, the inhibitory effect of MSTN on cell cycle progression [41], often interpreted as detrimental for myogenesis, may actually facilitate terminal differentiation $[42,45]$. The higher MSTN and MRF mRNA expression in COPD may therefore be a physiological response to promote terminal differentiation as part of an increased demand for myonuclear accretion. Importantly, several studies suggest that temporal expression of MSTN controls the balance between proliferation and differentiation of satellite cells $[41,46,47]$. A sustained elevation of MSTN expression may therefore impair satellite cell activation and negatively regulate self-renewal [41], eventually resulting in satellite cell senescence and depletion of the satellite cell pool $[42,48]$.

In conclusion, these results confirm increased protein degradation and synthesis signaling in the skeletal muscle of COPD patients compared with controls and show further stimulation of these processes in sarcopenic COPD patients compared with nonsarcopenic COPD patients. Together with the increase in myogenic signaling, this mirrors molecular alterations observed during muscle repair and remodeling (Figure 8). 

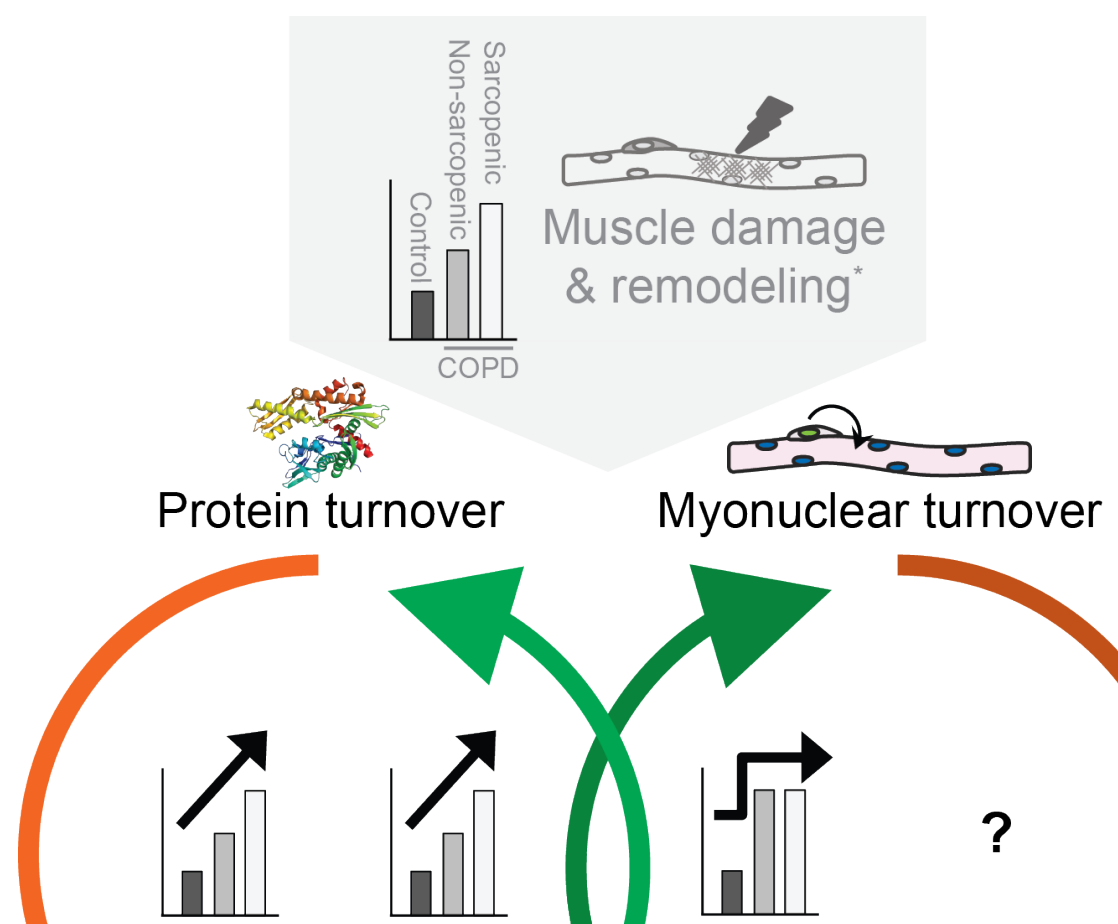

Autophagy

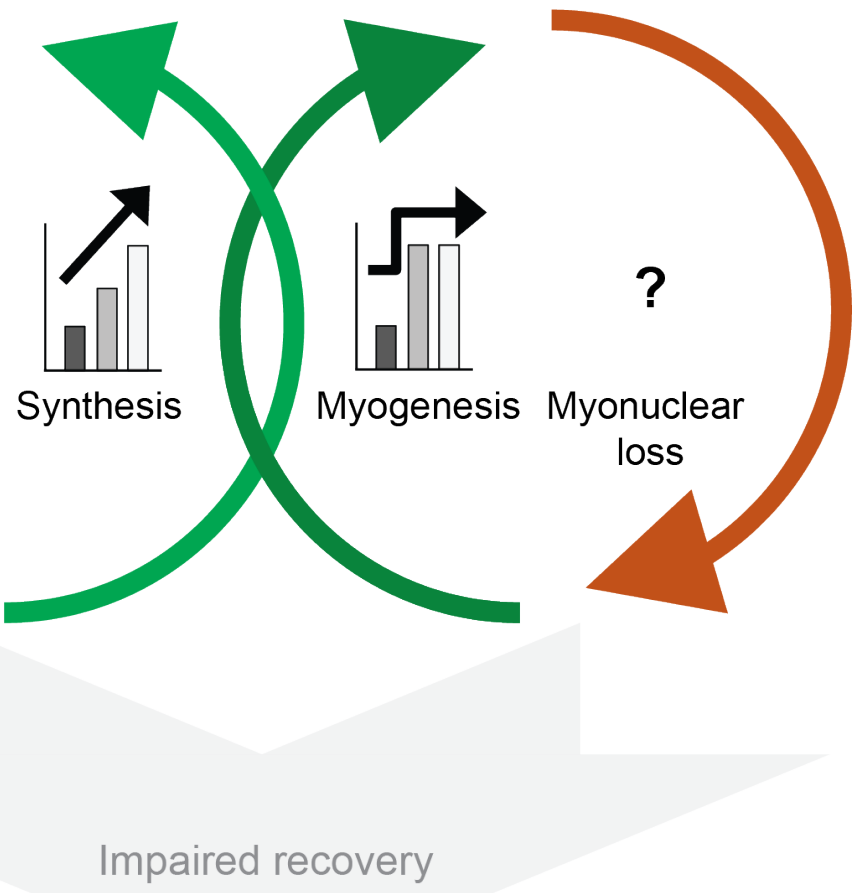

\section{Loss of muscle mass and function}

Figure 8. |Schematic presentation of sarcopenia-related molecular alterations in COPD. COPDrelated factors leading to muscle damage and remodeling may drive the increase in myogenic and protein turnover signaling in COPD, as well as the even further increased protein turnover signaling in sarcopenic COPD, which may impair recovery after an exacerbation, leading to a progressive loss of muscle mass and function. Black text represents the regulation of indicated processes as measured in the current study, whereas grey text represents their associations and implications from previous literature. *Puig-Vilanova et al. [29]. 


\section{CHAPTER 3}

\section{Implications and future perspectives}

Factors implicated in COPD-related sarcopenia, including oxidative stress and inflammation [32], can result from and cause cellular damage [49, 50], and may drive repair and remodeling-like molecular alterations. Although these molecular alterations are likely necessary to facilitate repair of myofiber damage, a sustained increase in protein turnover regulation elevates the energetic costs of protein metabolism [51], whereas a sustained increase in myogenic signaling could result in satellite cell depletion. This may not affect muscle mass substantially in a stable disease state, but it remains elusive to what extent this affects the coordination between anabolic signaling and the anabolic response required for muscle mass recovery after an exacerbation.

Future studies should therefore address the underlying mechanisms and pathophysiological relevance of repair and remodeling in the context of physiological responses to anabolic and catabolic stimuli in COPD patients, to elucidate the role of altered protein turnover and myogenic regulation in the development and treatment of COPD-related sarcopenia. 


\section{REFERENCES}

1. Cao L, Morley JE. Sarcopenia Is Recognized as an Independent Condition by an International Classification of Disease, Tenth Revision, Clinical Modification (ICD-10-CM) Code. J Am Med Dir Assoc 2016;17(8):675-7.

2. van de Bool C, Gosker HR, van den Borst B, et al. Muscle Quality is More Impaired in Sarcopenic Patients With Chronic Obstructive Pulmonary Disease. J Am Med Dir Assoc 2016;17(5):415-20.

3. Mostert R, Goris A, Weling-Scheepers C, et al. Tissue depletion and health related quality of life in patients with chronic obstructive pulmonary disease. Respir Med 2000;94(9):859-67.

4. Engelen MP, Schols AM, Baken WC, et al. Nutritional depletion in relation to respiratory and peripheral skeletal muscle function in out-patients with COPD. Eur Respir J 1994;7(10):17937.

5. Vestbo J, Prescott E, Almdal T, et al. Body mass, fat-free body mass, and prognosis in patients with chronic obstructive pulmonary disease from a random population sample: findings from the Copenhagen City Heart Study. Am J Respir Crit Care Med 2006;173(1):79-83.

6. Schols AM, Broekhuizen R, Weling-Scheepers CA, et al. Body composition and mortality in chronic obstructive pulmonary disease. The American journal of clinical nutrition 2005;82(1):53-9.

7. Maters GA, de Voogd JN, Sanderman R, et al. Predictors of all-cause mortality in patients with stable COPD: medical co-morbid conditions or high depressive symptoms. COPD 2014;11(4):468-74.

8. Fermoselle $C$, Rabinovich $\mathrm{R}$, Ausin $\mathrm{P}$, et al. Does oxidative stress modulate limb muscle atrophy in severe COPD patients? Eur Respir J 2012;40(4):851-62.

9. Vogiatzis I, Simoes DC, Stratakos G, et al. Effect of pulmonary rehabilitation on muscle remodelling in cachectic patients with COPD. Eur Respir J 2010;36(2):301-10.

10. Debigare R, Maltais $F$, Cote $\mathrm{CH}$, et al. Profiling of mRNA expression in quadriceps of patients with COPD and muscle wasting. COPD 2008;5(2):75-84.

11. Doucet $M$, Russell AP, Leger $B$, et al. Muscle atrophy and hypertrophy signaling in patients with chronic obstructive pulmonary disease. Am J Respir Crit Care Med 2007;176(3):261-9.

12. Lemire $B B$, Debigare $R$, Dube $A$, et al. MAPK signaling in the quadriceps of patients with chronic obstructive pulmonary disease. Journal of applied physiology 2012;113(1):159-66.

13. Plant PJ, Brooks D, Faughnan $M$, et al. Cellular markers of muscle atrophy in chronic obstructive pulmonary disease. Am J Respir Cell Mol Biol 2010;42(4):461-71.

14. Guo Y, Gosker HR, Schols AM, et al. Autophagy in locomotor muscles of patients with chronic obstructive pulmonary disease. Am J Respir Crit Care Med 2013;188(11):1313-20.

15. Lewis A, Riddoch-Contreras J, Natanek SA, et al. Downregulation of the serum response factor/miR-1 axis in the quadriceps of patients with COPD. Thorax 2012;67(1):26-34.

16. Engelen MP, Deutz NE, Wouters EF, et al. Enhanced levels of whole-body protein turnover in patients with chronic obstructive pulmonary disease. Am J Respir Crit Care Med 2000;162(4 Pt 1):1488-92.

17. Chung $\mathrm{JH}$, Hwang $\mathrm{HJ}$, Han $\mathrm{CH}$, et al. Association between sarcopenia and metabolic syndrome in chronic obstructive pulmonary disease: the Korea National Health and Nutrition Examination Survey (KNHANES) from 2008 to 2011. COPD 2015;12(1):82-9.

18. Jones SE, Maddocks M, Kon SS, et al. Sarcopenia in COPD: prevalence, clinical correlates and response to pulmonary rehabilitation. Thorax 2015;70(3):213-8.

19. Vermeeren MA, Creutzberg EC, Schols AM, et al. Prevalence of nutritional depletion in a large out-patient population of patients with COPD. Respir Med 2006;100(8):1349-55. 


\section{CHAPTER 3}

20. van den Borst B, Slot IG, Hellwig VA, et al. Loss of quadriceps muscle oxidative phenotype and decreased endurance in patients with mild-to-moderate COPD. Journal of applied physiology 2013;114(9):1319-28.

21. Cebron Lipovec N, Schols AM, van den Borst B, et al. Sarcopenia in Advanced COPD Affects Cardiometabolic Risk Reduction by Short-Term High-intensity Pulmonary Rehabilitation. Journal of the American Medical Directors Association 2016

22. Newman AB, Kupelian V, Visser M, et al. Sarcopenia: alternative definitions and associations with lower extremity function. J Am Geriatr Soc 2003;51(11):1602-9.

23. Miller MR, Crapo R, Hankinson J, et al. General considerations for lung function testing. Eur Respir J 2005;26(1):153-61.

24. Vestbo J, Hurd SS, Agusti AG, et al. Global Strategy for the Diagnosis, Management, and Prevention of Chronic Obstructive Pulmonary Disease GOLD Executive Summary. American journal of respiratory and critical care medicine 2013;187(4):347-65.

25. Franssen FM, Sauerwein HP, Ackermans MT, et al. Increased postabsorptive and exerciseinduced whole-body glucose production in patients with chronic obstructive pulmonary disease. Metabolism 2011;60(7):957-64.

26. Milan G, Romanello V, Pescatore $F$, et al. Regulation of autophagy and the ubiquitinproteasome system by the FoxO transcriptional network during muscle atrophy. Nat Commun 2015;6:6670.

27. Lapierre LR, Kumsta C, Sandri M, et al. Transcriptional and epigenetic regulation of autophagy in aging. Autophagy 2015;11(6):867-80.

28. Hesselink MK, Minnaard R, Schrauwen P. Eat the meat or feed the meat: protein turnover in remodeling muscle. Current opinion in clinical nutrition and metabolic care 2006;9(6):6726. [published Online First: 2006/10/21]

29. Puig-Vilanova E, Rodriguez DA, Lloreta J, et al. Oxidative stress, redox signaling pathways, and autophagy in cachectic muscles of male patients with advanced COPD and lung cancer. Free Radic Biol Med 2015;79:91-108.

30. Natanek SA, Riddoch-Contreras J, Marsh GS, et al. MuRF-1 and atrogin-1 protein expression and quadriceps fiber size and muscle mass in stable patients with COPD. COPD 2013;10(5):618-24.

31. Atherton PJ, Etheridge T, Watt PW, et al. Muscle full effect after oral protein: time-dependent concordance and discordance between human muscle protein synthesis and mTORC1 signaling. Am J Clin Nutr 2010;92(5):1080-8.

32. Langen RC, Gosker HR, Remels AH, et al. Triggers and mechanisms of skeletal muscle wasting in chronic obstructive pulmonary disease. The international journal of biochemistry \& cell biology 2013;45(10):2245-56.

33. Sacheck JM, Hyatt JP, Raffaello A, et al. Rapid disuse and denervation atrophy involve transcriptional changes similar to those of muscle wasting during systemic diseases. FASEB journal : official publication of the Federation of American Societies for Experimental Biology 2007;21(1):140-55. [published Online First: 2006/11/23]

34. Hopkinson NS, Tennant RC, Dayer MJ, et al. A prospective study of decline in fat free mass and skeletal muscle strength in chronic obstructive pulmonary disease. Respir Res 2007;8:25.

35. Alahmari AD, Kowlessar BS, Patel AR, et al. Physical activity and exercise capacity in patients with moderate COPD exacerbations. Eur Respir J 2016;48(2):340-49.

36. Michailidis Y, Karagounis LG, Terzis G, et al. Thiol-based antioxidant supplementation alters human skeletal muscle signaling and attenuates its inflammatory response and recovery after intense eccentric exercise. Am J Clin Nutr 2013;98(1):233-45.

37. Nichenko AS, Southern WM, Atuan M, et al. Mitochondrial maintenance via autophagy contributes to functional skeletal muscle regeneration and remodeling. American journal 
of physiology Cell physiology 2016:ajpcell 000662016.

38. Cermak NM, Snijders T, McKay BR, et al. Eccentric exercise increases satellite cell content in type II muscle fibers. Medicine and science in sports and exercise 2013;45(2):230-7.

39. Theriault $M E$, Pare $M E$, Lemire $B B$, et al. Regenerative defect in vastus lateralis muscle of patients with chronic obstructive pulmonary disease. Respiratory research 2014;15:35.

40. Trendelenburg AU, Meyer A, Rohner D, et al. Myostatin reduces Akt/TORC1/p70S6K signaling, inhibiting myoblast differentiation and myotube size. Am J Physiol Cell Physiol 2009;296(6):C1258-70.

41. McCroskery $S$, Thomas $M$, Maxwell $L$, et al. Myostatin negatively regulates satellite cell activation and self-renewal. J Cell Biol 2003;162(6):1135-47.

42. Manceau M, Gros J, Savage $K$, et al. Myostatin promotes the terminal differentiation of embryonic muscle progenitors. Genes \& development 2008;22(5):668-81.

43. Langley B, Thomas M, Bishop A, et al. Myostatin inhibits myoblast differentiation by downregulating MyoD expression. J Biol Chem 2002;277(51):49831-40.

44. Steelman CA, Recknor JC, Nettleton D, et al. Transcriptional profiling of myostatin-knockout mice implicates Wnt signaling in postnatal skeletal muscle growth and hypertrophy. FASEB journal : official publication of the Federation of American Societies for Experimental Biology 2006;20(3):580-2.

45. Bernardi H, Gay S, Fedon Y, et al. Wnt4 activates the canonical beta-catenin pathway and regulates negatively myostatin: functional implication in myogenesis. Am J Physiol Cell Physiol 2011;300(5):C1122-38.

46. Rossi G, Antonini S, Bonfanti C, et al. Nfix Regulates Temporal Progression of Muscle Regeneration through Modulation of Myostatin Expression. Cell Rep 2016;14(9):2238-49.

47. George RM, Biressi S, Beres BJ, et al. Numb-deficient satellite cells have regeneration and proliferation defects. Proc Natl Acad Sci U S A 2013;110(46):18549-54.

48. Le Grand F, Rudnicki MA. Skeletal muscle satellite cells and adult myogenesis. Current opinion in cell biology 2007;19(6):628-33.

49. Chen GY, Nunez G. Sterile inflammation: sensing and reacting to damage. Nature reviews Immunology 2010;10(12):826-37.

50. Demple B, Harrison L. Repair of oxidative damage to DNA: enzymology and biology. Annu Rev Biochem 1994;63:915-48.

51. Bier DM. The Role of Protein and Amino Acids in Sustaining and Enhancing Performance. The Energy Costs of Protein Metabolism: Lean and Mean on Uncle Sam's Team: Washington (DC): National Academies Press (US), 1999. 


\section{CHAPTER 3}

\section{SUPPLEMENTARY MATERIAL}

\section{METHODS}

\section{$R T-q P C R$}

Tissue was homogenized (Beat-Beater) in presence of TRI-reagent (Sigma), and RNA was isolated by TRI-reagent/Chloroform extraction and subsequently precipitated from the aqueous phase using glycogen-containing isopropanol. RNA was reconstituted in $30 \mu \mathrm{l}$ RNA storage solution (Ambion AM7001) and stored at $-80^{\circ} \mathrm{C}$. RNA concentrations were measured spectrophotometrically using a Nanodrop UV-Vis spectrophotometer (ND-1000, Thermo Scientific). 400 ng RNA was diluted in nuclease free $\mathrm{H}_{2} \mathrm{O}$ and reverse transcribed to cDNA using the Tetro cDNA synthesis kit (Bioline) according to the manufacturer's instructions. qPCR primers were designed based on Ensembl transcript sequences and ordered from Sigma Genosys (Zwijndrecht, the Netherlands), with primer details shown in Table S1. qPCR reactions contained Sensimix SYBR \& ROX (GC Biotech) and primer mix, and were run in a 384 well white opaque plate (Roche) on a LightCycler 480 system (Roche). Melting curves were analysed to verify specificity of the amplification, and relative quantity of the targets was assessed by LinRegPCR software (v2014.8) [1]. Three reference genes (RPLP0, B2M, and PPIA) were used to calculate a GeNorm correction factor, which was used to normalize expression levels of the target genes.

\section{Western Blot}

Tissue was homogenized in $600 \mu \mathrm{l}$ of Immunoprecipitation lysis buffer (50mM Tris, $150 \mathrm{mM} \mathrm{NaCl}$, 10\% glycerol, 0.5\% Nonidet P40, protease and phosphatase inhibitors (Roche)) with a Micro Tissue Homogenizer. After homogenization, samples were incubated on a tube rotator at $4^{\circ} \mathrm{C}$ for 30 minutes and centrifuged at $14000 \mathrm{~g}$ at $4^{\circ} \mathrm{C}$ for 30 minutes. The supernatant was stored at $-80^{\circ} \mathrm{C}$ until analysis. Total protein concentration was determined using BCA Protein Assay kit (Pierce) according to the manufacturer's instructions. For Western Blot analyses, 4x Laemmli sample buffer (0.25M Tris-HCl ph6.8; 8\% (w/v) SDS; 40\% (v/v) glycerol; 0.4M DTT and 0.02\% (w/v) Bromophenol Blue) was added and samples were heated to $100^{\circ} \mathrm{C}$ for 5 minutes. 10 $\mu \mathrm{g}$ protein was separated on a Criterion XT Precast 4-12\% Bis-Tris gel (Bio-Rad) in XT MOPS running buffer (Bio-Rad) by gel electrophoresis. Proteins were transferred to a nitrocellulose membrane (Bio-Rad) by electroblotting at $100 \mathrm{~V}$ for 60 minutes in transfer buffer (25 mM Tris, 192 mM Glycine, 20\% (vol/vol) methanol).

For total protein detection, the membrane was stained with PonceauS solution $(0.2 \%$ PonceauS in 1\% acetic acid; Sigma-Aldrich Chemie) and imaged using the Amersham 
imager 600RGB. The membrane was blocked for 1 hour at room temperature (RT) in Tris-buffered saline with Tween20 (TBST; $20 \mathrm{mM}$ Tris, $137 \mathrm{mM} \mathrm{NaCl}, 0.1 \%$ (vol/vol) Tween20, pH 7.6) containing 3\% (w/v) nonfat dry milk (Campina, Eindhoven, the Netherlands). The membranes were washed in TBST, followed by overnight incubation at $4^{\circ} \mathrm{C}$ with primary antibody diluted in TBST with $3 \%$ BSA or non-fat dry milk (Table S2). Membranes were incubated with a peroxidase conjugated secondary antibody solution (Vector Laboratories, \#PI-1000 (Diluted 1:10000 in blocking solution)) for 1 hour at RT, and targets were visualized by chemiluminescence using Supersignal West PICO or FEMTO Chemiluminescent Substrate (Pierce Biotechnology, Inc.) according to the manufacturer's instructions, and detected using the Amersham imager 600RGB. Signals were quantified with Image Quant software (Amersham). For analyses, samples from controls and COPD patients were randomly distributed within and between blots, and reference samples were loaded onto all blots to correct for between-blot variation. Protein expression and phosphorylation levels were corrected for total protein content, and presented as a fold change compared to the control group.

\section{REFERENCES}

1. Ramakers C, Ruijter JM, Deprez RH, et al. Assumption-free analysis of quantitative real-time polymerase chain reaction (PCR) data. Neurosci Lett 2003;339(1):62-6. 


\section{CHAPTER 3}

Table S1. | qPCR primer sequences of genes of interest and reference genes

\begin{tabular}{|c|c|c|c|c|}
\hline Target & $\mathbf{N}^{*}$ & Source & FW & REV \\
\hline FOXO1 & $13 / 51 / 38$ & ENSG00000150907 & CCTGGACATGCTCAGCAGACATC & TTGGGTCAGGCGGTTCATACC \\
\hline FOXO3 & $13 / 51 / 38$ & ENSG00000118689 & CACTGGTGCTAAGCAGGCCTCAT & ATGGCGTGGGATTCACAAAGGT \\
\hline MURF1 & $13 / 51 / 38$ & ENSG00000158022 & GCGAGGTGGCCCCATT & GATGGTCTGCACACGGTCATT \\
\hline MUSA1 & $13 / 50 / 38$ & ENSG00000118496 & AGTACTCCTGACAGCAGCAG & CATTGACACAGAAGTCAATCACTC \\
\hline SMART & $13 / 50 / 38$ & ENSG00000135108 & AATTAATCTGAAAGGCACTGTGTC & TGAAGACAGAATGTCACAAACTG \\
\hline ATROGIN1 & $13 / 51 / 38$ & ENSG00000156804 & GAAGAAACTCTGCCAGTACCACTTC & CCCTTIGTCTGACAGAATTAATCG \\
\hline$\angle C 3 B$ & $13 / 51 / 38$ & ENSG00000140941 & ACCATGCCGTCGGAGAAGAC & TCTCGAATAAGTCGGACATCTTCTACTCT \\
\hline P62 & $13 / 51 / 38$ & ENSG00000161011 & GGTGCACCCCAATGTGATCT & CGCAGACGCTACACAAGTCG \\
\hline$B E C N 1$ & $13 / 51 / 38$ & ENSG00000126581 & AATGCAACCTTCCACATCTGGC & CCCAGCCTGAAGTTATTGATTGTGC \\
\hline ULK1 & $13 / 50 / 38$ & ENSG00000177169 & TGCCCGGTGTCCTGGTCCTC & CAGGGGCAGCAGTTGAGGCG \\
\hline PAX7 & $13 / 50 / 38$ & ENSG00000009709 & TACAGCACCACCGGCTACAGCGTG & CTGGCCGTACTGGCCGTACTGATAG \\
\hline PCNA & $13 / 50 / 38$ & ENSG00000132646 & TCTGCAAGTGGAGAACTTGGAA & GAAGTTCAGGTACCTCAGTGCAA \\
\hline CCND1 & $13 / 50 / 38$ & ENSG00000110092 & ACCGCCTCACACGCTTCCTCT & GCCTGGCGCAGGCTTGACTC \\
\hline MYF5 & $13 / 50 / 38$ & ENSG00000111049 & TGCCCGAATGTAACAGTCCT & AGCAATCCAAGCTGGATAAGG \\
\hline MYOD1 & $13 / 51 / 38$ & ENSG00000129152 & CAATCCAAACCAGCGGTTGC & CCTCGATATAGCGGATGGCGT \\
\hline MYOG & $13 / 51 / 38$ & ENSG00000122180 & TCAGCGCCAACCCAGG & GGTGAGGGAGTGCAGGTTGT \\
\hline M-cadherin & $13 / 50 / 38$ & ENSG00000129910 & CCTGGACATCGCCGACTTCA & TGGGGTCACTATCTGCAGCC \\
\hline MSTN & $13 / 51 / 38$ & ENSG00000138379 & AACCTTCCCAGGACCAGGAGAA & TGTCTGTTACCTTGACCTCTAAAAACGG \\
\hline RPLPO & $13 / 51 / 38$ & ENSG00000089157 & TCTACAACCCTGAAGTGCTTGATATC & GCAGACAGACACTGGCAACATT \\
\hline$B 2 M$ & $13 / 51 / 38$ & ENSG00000196262 & CATCTGCACTGCCAAGACTGA & ТTСATGCCTТСТTСАСТTTGC \\
\hline PPIA & $13 / 51 / 38$ & ENSG00000166710 & CTGTGCTCGCGCTACTCTCTCTT & TGAGTAAACCTGAATCTTGGAGTACGC \\
\hline
\end{tabular}

*Sample size (controls/non-sarcopenic COPD patients/sarcopenic COPD patients). Abbreviations: FOXO1, forkhead box O1; FOXO3, forkhead box O3; MURF1 (TRIM63), tripartite motif containing 63; MUSA1 (FBXO30), F-box protein 30; SMART (FBXO21), F-box protein 21; ATROGIN1 (FBXO32/ $M A F B X), F-b o x$ protein 32; LC3B (MAP1LC3B), microtubule associated protein 1 light chain 3 beta; P62 (SQSTM1), sequestosome 1; BECN1, Beclin1; ULK1, unc-51 like autophagy activating kinase 1; PAX7, paired box 7; PCNA, proliferating cell nuclear antigen; CCND1, cyclin D1; MYF5, myogenic factor 5; MYOD1, myogenic differentiation 1; MYOG, myogenin; M-cadherin (CDH15), cadherin 15; MSTN, myostatin; RPLPO, ribosomal protein lateral stalk subunit PO; B2M, beta-2-microglobulin; PPIA, peptidylprolyl isomerase $A$. 
Table S2. | Primary antibody list

\begin{tabular}{|c|c|c|c|}
\hline Target & $\mathbf{N}^{*}$ & Supplier & Product nr. \\
\hline FOXO1 & $12 / 52 / 38$ & Cell signaling & 2880 \\
\hline FOXO3 & $12 / 52 / 38$ & Cell signaling & 2497 \\
\hline FOXO1 (Tyr24)/FOXO3(Tyr32) & $11 / 52 / 38$ & Cell signaling & 9464 \\
\hline LC3B & $12 / 52 / 38$ & Cell signaling & 2775 \\
\hline P62 & $12 / 53 / 38$ & Cell signaling & 5114 \\
\hline BECN1 & $12 / 53 / 38$ & Novus Biologicals & NB110-87318SS \\
\hline ULK1 & $12 / 53 / 38$ & Cell signaling & 8054 \\
\hline ULK1(Ser757) & $12 / 53 / 38$ & Cell signaling & 6888 \\
\hline ULK1(Ser555) & $12 / 53 / 38$ & Cell signaling & 5869 \\
\hline AKT1 & $12 / 53 / 39$ & Cell signaling & 2920 \\
\hline AKT(Ser473) & $12 / 53 / 39$ & Cell signaling & 4060 \\
\hline MTOR & $11 / 53 / 39$ & Cell signaling & 2983 \\
\hline MTOR(Ser2448) & $11 / 53 / 39$ & Cell signaling & 2971 \\
\hline GSK3B & $12 / 53 / 39$ & Cell signaling & 12456 \\
\hline GSK3B(Ser9) & $12 / 53 / 39$ & Cell signaling & 9336 \\
\hline $4 \mathrm{E}-\mathrm{BP} 1$ & $12 / 53 / 39$ & Cell signaling & 9452 \\
\hline 4E-BP1(Ser65) & $12 / 53 / 39$ & Cell signaling & 9451 \\
\hline RPS6 & $10 / 53 / 39$ & Cell signaling & 2317 \\
\hline RPS6(Ser235/236) & $10 / 53 / 39$ & Cell signaling & 4858 \\
\hline
\end{tabular}

*Sample size (controls/non-sarcopenic COPD patients/sarcopenic COPD patients). Abbreviations: FOXO1, forkhead box O1/ FOXO3, forkhead box O3/ LC3B, microtubule associated protein 1 light chain 3 beta/ P62, sequestosome 1/ BECN1, Beclin 1/ ULK1, unc-51 like autophagy activating kinase 1/ AKT1, AKT serine/threonine kinase 1/ MTOR, mechanistic target of rapamycin/ GSK3B, glycogen synthase kinase 3 beta/ 4E-BP1, eukaryotic translation initiation factor $4 E$ binding protein 1/ RPS6, ribosomal protein S6/Tyr, tyrosine/Ser, Serine. 



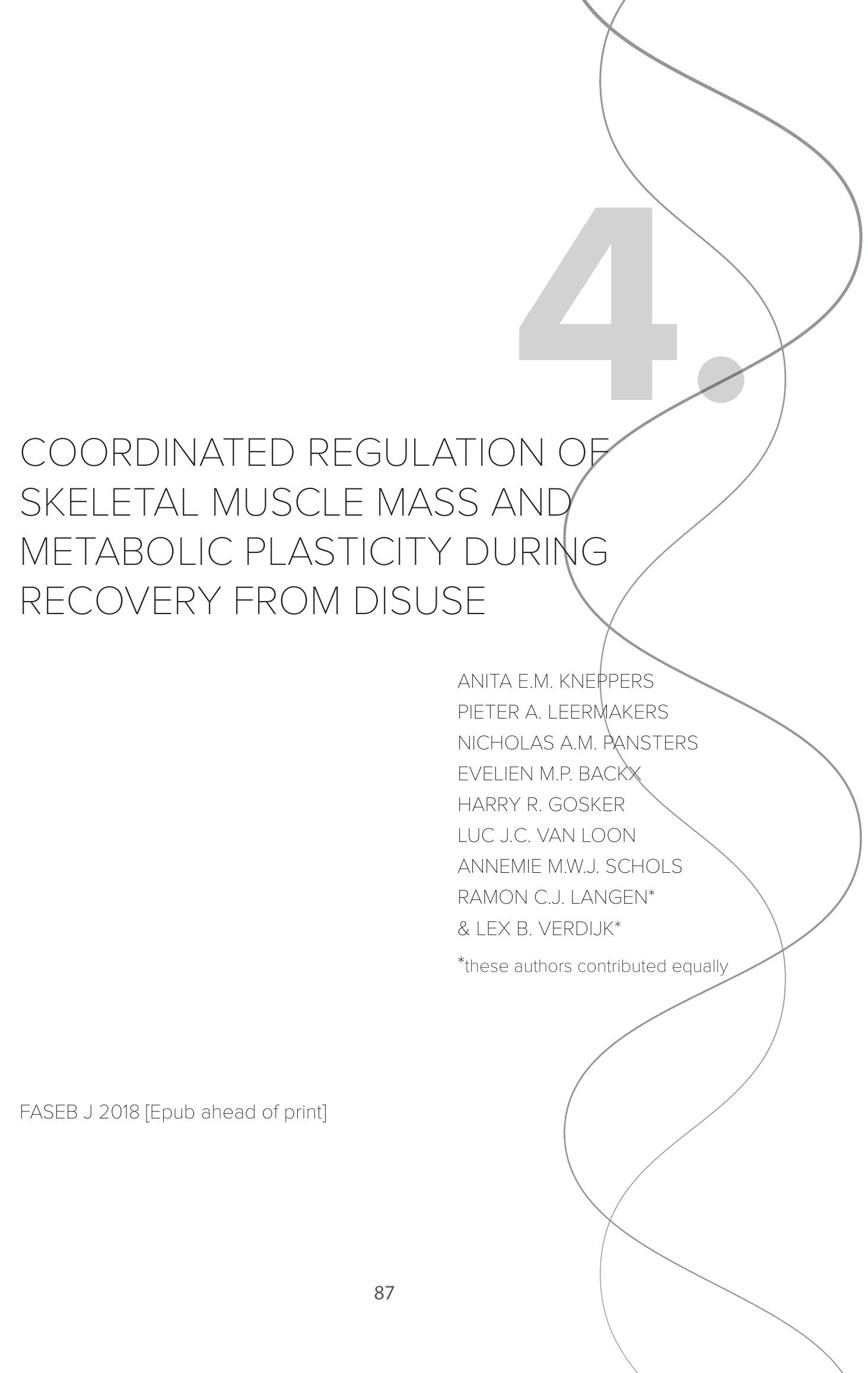




\section{ABSTRACT}

Background: Skeletal muscle regeneration after disuse is essential for muscle maintenance and involves the regulation of both mass- and metabolic plasticityrelated processes. However, the relation between these processes during recovery from disuse remains unclear. In this study, we explored the potential interrelationship between the molecular regulation of muscle mass and oxidative metabolism during recovery from disuse.

Methods: Molecular profiles were measured in biopsies from the vastus lateralis of healthy men after 1-leg cast immobilization and after $1 \mathrm{wk}$ reloading, and in mouse gastrocnemius obtained before and after hindlimb suspension and during reloading $(R L-1,-2,-3,-5$, and $-8 d)$.

Results: Cluster analysis of the human recovery response revealed correlations between myogenesis and autophagy markers in 2 clusters, which were distinguished by the presence of markers of early myogenesis, autophagosome formation, and mitochondrial turnover vs. markers of late myogenesis, autophagy initiation, and mitochondrial mass. In line with these findings, an early transient increase in B-cell lymphoma- 2 interacting protein 3 and sequestosome- 1 protein, and GABA type A receptor-associated protein like-1 protein and mRNA and a late increase in myomaker and myosin heavy chain-8 mRNA, microtubule-associated protein 1 light chain 3-II:I ratio, and FUN14 domain-containing-1 mRNA and protein were observed in mice.

Conclusion: The regulatory profiles of protein, mitochondrial, and myonuclear turnover are correlated and temporally associated, suggesting a coordinated regulation of muscle mass- and oxidative metabolism-related processes during recovery from disuse.

\section{Acknowledgements}

The authors thank Marco Kelders, Chiel de Theije, and Antoine Zorenc for their excellent technical assistance in performing the molecular analyses, and Roy Haast for his support in conducting the cluster analysis. 


\section{INTRODUCTION}

Skeletal muscles are essential to generate the forces for postural support and physical function. Furthermore, representing half of the body mass and being highly metabolically active, skeletal muscle tissue is an important site for control of metabolism [1]. These functions are supported by the muscles' biochemical and morphologic makeup, which are tightly regulated and can rapidly adapt to alterations in mechanical and metabolic demands - referred to as skeletal muscle plasticity.

Muscle disuse results from low skeletal muscle mechanical loading, such as occurs with bed rest during hospitalization after surgery or acute illness, with immobilization after a fracture, and with certain chronic diseases. It is well established that disuse leads to a reduction of skeletal muscle strength [2] and mass [2, 3], which results from a negative balance in muscle protein turnover $[2,4]$. In addition, disuse affects muscle quality, including a transition from a slow to fast fiber type [5-7], a shift from fat oxidation toward glycolysis [8], a decrease in mitochondrial mass and function [9-12], and a reduction in vascularization [13].

The above-mentioned qualitative alterations in response to disuse collectively reduce the capacity for oxidative metabolism. It has been suggested that these metabolic alterations represent an adaptation to a decreased energy requirement resulting from a reduction in the level of protein turnover upon disuse [8]. On the other hand, a study showed that the inhibition of disuse-induced mitochondrial alterations prevents the loss of muscle mass [12]. These findings indicate that muscle disuse-induced mass and metabolic alterations are highly interdependent.

Although disuse-induced alterations in skeletal muscle mass and function may be part of a normal physiologic response, the negative implications of muscle disuse atrophy for general health, quality of life [14], and even survival [15-17] have been clearly demonstrated. Both nutritional interventions and exercise mimetics have been used to prevent muscle disuse atrophy, but none serve as a complete substitute for the mechanical and metabolic stimulation during normal muscle use [18-22]. Nevertheless, in otherwise healthy individuals, a normalization of muscle mass and function occurs upon reloading after disuse $[23,24]$. However, the negative consequences of disuse may persist when muscle regeneration is impaired, as occurs in elderly [25-27] and chronically ill persons [28]. To identify and target factors that hamper skeletal muscle recovery during resumed physical activity after disuse in such persons, a better understanding of the molecular alterations driving skeletal muscle plasticity during reloading-induced recovery is imperative. 


\section{CHAPTER 4}

In contrast to the wealth of data on the molecular alterations during skeletal muscle disuse in both humans and animals, insight into the molecular response to reloading to induce skeletal muscle regeneration is less abundant and depends mainly on data from studies in rodents. From these studies, it is clear that protein synthesis signaling related to the control of mRNA translation is increased upon reloading after disuse [27, $29,30]$ and that markers of the ubiquitin proteasome system (UPS) return to baseline levels during reloading [24, 30]. Rather than a passive normalization of the protein turnover balance, several studies suggest a transient activation of protein degradation signaling during reloading-induced recovery, particularly of the autophagy-lysosome pathway $[24,27,31,32]$. In addition, myogenesis seems to be activated during skeletal muscle reloading $[4,30,33]$.

These reloading-induced molecular alterations are also implicated in intrinsic remodeling of skeletal muscle. Indeed, metabolic alterations take place upon skeletal muscle reloading, including an activation of processes that regulate mitochondrial biogenesis and function [32, 34, 35] and an increase in mitochondrial mass [32, 34]. Similar to the interdependency of mass and metabolic alterations upon muscle disuse, these processes may be linked during reloading-induced recovery. However, such a potential relation is largely understudied. In the present study, we therefore sought to provide insight in the interrelationship between the regulation of muscle mass and muscle metabolic plasticity during recovery from disuse. A better understanding of these relations is essential for the identification of the molecular origin, and subsequent treatment, of impaired remobilization-induced skeletal muscle recovery in the elderly and chronically ill. We explored these relations by measuring a selected set of indicators and molecular regulators and mediators of muscle mass- and oxidative metabolism-related processes and studied their interrelationship during reloadinginduced recovery after disuse in both humans and rodents.

\section{METHODS}

\section{Subjects, study design, and measurements}

Skeletal muscle molecular profiles of 14 healthy young men (age, $23 \pm 1 \mathrm{yr}$; body mass index, $22.9 \pm 0.6 \mathrm{~kg} / \mathrm{m}^{2}$ ) who participated in a double-blind, randomized, placebo-controlled intervention trial (www.clinicaltrials.gov; NCT01894737) were analyzed. Written informed consent was obtained from all subjects, and the study was approved by the Maastricht University Medical Center+ (Maastricht, the Netherlands) ethics review board (13-3-023) and performed in accordance with the Declaration of Helsinki. The study design and primary data were published previously [36]. In brief, 
participants were subjected to $7 \mathrm{~d}$ of 1-leg immobilization by means of a full leg cast [unloading (UL)], followed by $7 \mathrm{~d}$ of remobilization [reloading (RL)]. Subjects were asked to maintain habitual dietary intake and refrain from any heavy physical exercise during the entire intervention period. Their compliance was checked and confirmed by dietary intake records and activity journals. Furthermore, the subjects consumed standardized meals the evening before all test days. Needle biopsies from the vastus lateralis of the casted leg were taken after an overnight fast after UL and again after RL. Samples were frozen in liquid nitrogen and stored at $-80^{\circ} \mathrm{C}$ until further analysis. Furthermore, muscle mass, measured by cross-sectional area (CSA) was assessed by a computed tomographic scan of the upper legs, muscle strength was assessed with a 1-repetition maximum (1-RM) knee extension test, and body weight was measured with a digital scale. None of the subjects performed progressive resistance-type exercise training or took creatine supplements or any medication that would interfere with muscle metabolism in the 6 mo before the study.

Only subjects with a complete set of available muscle biopsies were included in the current subanalysis $(n=14)$, of whom 5 received placebo and 9 received creatine supplementation. Based on an increase of $<10 \mathrm{mM} / \mathrm{kg}$ in muscle total creatine content [36], 4 subjects were considered nonresponders to creatine loading. Furthermore, creatine supplementation did not have any impact on muscle disuse or subsequent recovery [36].

\section{Animals and study design}

The animal study was approved by the Institutional Animal Care Committee of Maastricht University (DEC-2009-074). The study design has been published [30,

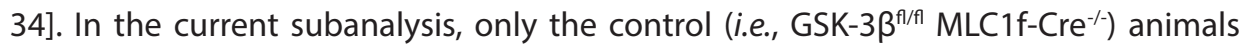
were used. In brief, 13-wk-old male C57/Bl6 mice were housed in a temperaturecontrolled room $\left(21-22^{\circ} \mathrm{C}\right)$ with a $12-12 \mathrm{~h}$ light-dark cycle and standard chow pellets and water ad libitum. Mice were subjected to either no experimental procedure (baseline, BL; $\mathrm{n}=9$ ), $14 \mathrm{~d}$ of muscle $U L$ by hindlimb suspension ( $U L, n=8$ ), or $1,2,3,5$, or $8 \mathrm{~d}$ of RL after hindlimb suspension ( $\mathrm{n}=8$ per time point). Mice were euthanized at each time point by injection with pentobarbital sodium, and hindlimb muscles were excised with standardized dissection methods. Muscles were cleaned of excess fat and connective tissue, pair weighed on an analytical scale, and snap frozen in liquid nitrogen (biochemical analyses) or embedded in Tissue-Tek optimal cutting temperature compound (Sakura Finetek, Zoeterwolde, the Netherlands) and frozen in melting isopentane (histochemical analyses). Samples were stored at $-80^{\circ} \mathrm{C}$ until further analysis. The gastrocnemius was used for protein and mRNA analyses, as its 


\section{CHAPTER 4}

response to hindlimb UL is pronounced and well characterized in studies [37-39]. Furthermore, it has a fiber type composition and distribution that is representative of many muscles in the hindlimb and is similar to the fiber type composition observed in the human vastus lateralis.

\section{Protein isolation and Western blot analysis}

For protein analysis, frozen mouse gastrocnemius was powdered and lysed in a wholecell lysis buffer (50 mM Tris, $150 \mathrm{mM} \mathrm{NaCl}, 10 \%$ glycerol, 0.5\% Nonidet P40, 1 mM EDTA, $500 \mu \mathrm{M} \mathrm{Na}_{3} \mathrm{VO}_{4^{\prime}} 500 \mu \mathrm{M} \mathrm{NaF}, 100 \mu \mathrm{M} \beta$-glycerophosphate, $100 \mu \mathrm{M} \mathrm{Na}_{4} \mathrm{P}_{2} \mathrm{O}_{7^{\prime}} 1 \mathrm{mM}$ DTT, $10 \mu \mathrm{g} / \mathrm{ml}$ leupeptin, and $1 \%$ aprotinin) for $1 \mathrm{~h}$ on a tube rotator at $4^{\circ} \mathrm{C}$, followed by 30 min of centrifugation at $14,000 \mathrm{~g}$ at $4^{\circ} \mathrm{C}$. Lysates were aliquoted in sample buffer $[0.25 \mathrm{M}$ Tris- $\mathrm{HCl}, 8 \%(\mathrm{w} / \mathrm{v})$ SDS, 40\% (v/v) glycerol, 0.4M DTT, and 0.02\% (w/v) bromophenol blue], boiled for $5 \mathrm{~min}$ at $95^{\circ} \mathrm{C}$, and stored at $-80^{\circ} \mathrm{C}$. Human vastus lateralis biopsies were homogenized in lysis buffer (20 mM Tris- $\mathrm{HCl}$, 1\% Nonidet P40, 5 mM EDTA, 2 $\mathrm{mM} \mathrm{Na}_{3} \mathrm{VO}_{4^{\prime}} 100 \mathrm{mM} \mathrm{NaF}, 10 \mathrm{mM} \mathrm{Na}{ }_{4} \mathrm{P}_{2} \mathrm{O}_{7}, 10 \mu \mathrm{g} / \mathrm{ml}$ leupeptin, $10 \mu \mathrm{g} / \mathrm{ml}$ aprotinin, 3 $\mathrm{mM}$ benzamidine, and $1 \mathrm{mM}$ PMSF) using an Ultra-Turrax (IKA Works, Wilmington, NC, USA). Lysates were centrifuged for $10 \mathrm{~min}$ at $10,000 \mathrm{~g}$ at $4^{\circ} \mathrm{C}$. Lysates were aliquoted in sample buffer [0.35M Tris- $\mathrm{HCl}, 10 \%(\mathrm{w} / \mathrm{v}) \mathrm{SDS}, 30 \%(\mathrm{v} / \mathrm{v})$ glycerol, $0.6 \mathrm{M} \mathrm{DTT}$, and $0.02 \%$ bromophenol blue], boiled for $5 \mathrm{~min}$ at $100^{\circ} \mathrm{C}$, and stored at $-80^{\circ} \mathrm{C}$. Total protein concentration was determined with the Pierce BCA Protein Assay Kit (Thermo Fisher Scientific, Waltham, MA, USA), according to the manufacturer's instructions.

Ten micrograms protein was separated on a Criterion XT Precast Bis-Tris Gel (Bio-Rad, Hercules, CA, USA) in XT-3-[N-morpholino]propanesulfonic acid or XT-3-[N-morpholino] ethanesulfonic acid running buffer (Bio-Rad) by gel electrophoresis. Proteins were transferred to a $0.45-\mu \mathrm{m}$ nitrocellulose membrane (Bio-Rad) by electroblotting at $100 \mathrm{~V}$ for $60 \mathrm{~min}$ in transfer buffer [25 mM Tris, $192 \mathrm{mM}$ glycine, 20\% (v/v) methanol]. For total protein detection, membranes were stained with PonceauS solution ( $0.2 \%$ PonceauS in 1\% acetic acid; MilliporeSigma, Burlington, MA, USA) for 3-5 min, washed in doubledistilled $\mathrm{H}_{2} \mathrm{O}$ for $30 \mathrm{~s}$ on a shaker, and imaged with the Amersham imager 600RGB (GE Healthcar, Chicago, IL, USA). The membranes were blocked for $1 \mathrm{~h}$ at room temperature in Tris-buffered saline with Tween 20 [TBST; $20 \mathrm{mM}$ Tris, $137 \mathrm{mM} \mathrm{NaCl}, 0.1 \%$ (v/v) Tween 20, pH 7.6] containing 5\% (w/v) nonfat dry milk (Campina, Eindhoven, the Netherlands). The membranes were washed in TBST, followed by overnight incubation at $4^{\circ} \mathrm{C}$ with primary antibody diluted in TBST with 3\% bovine serum albumin (Supplemental Table S1). After a wash in TBST, membranes were incubated with a peroxidase-conjugated secondary antibody solution (Vector Laboratories, Burlingame, CA, USA) for $1 \mathrm{~h}$ at room temperature and targets were visualized by chemiluminescence (Supersignal 
West PICO or FEMTO Chemiluminescent substrate; Thermo Fisher Scientific), according to the manufacturer's instructions, and detected with the Amersham Imager 600RGB. Signals were quantified with Image Quant TL software (GE Healthcare). Samples from each group were distributed within and between blots, to allow for correction of between-session variation [40], and protein expression and phosphorylation levels were corrected for protein loading assessed by PonceauS.

\section{RNA isolation and $R T-q P C R$}

For RNA analysis, powdered mouse gastrocnemius was lysed in RLT solution containing 1\% 2-ME. Total RNA was extracted using an on-column RNA isolation kit (Qiagen, Hilden, Germany) according to the manufacturer's instructions and stored at $-80^{\circ} \mathrm{C}$. Human vastus lateralis biopsies were lysed in Trizol (Thermo Fisher Scientific). Total RNA was isolated by phenol-chloroform extraction and subsequent precipitation from the aqueous phase with glycogen-containing isopropanol. RNA was reconstituted in nuclease-free $\mathrm{H}_{2} \mathrm{O}$ and stored at $-80^{\circ} \mathrm{C}$.

RNA concentration was determined with a spectrophotometer (Nanodrop ND-1000 UV-Vis; Thermo Fisher Scientific). RNA (400 ng/sample) was reverse transcribed into cDNA with a Transcriptor cDNA synthesis kit (Roche, Basel, Switzerland), according to the manufacturer's instructions. Primers were designed based on Ensemble transcript sequences and ordered from MilliporeSigma, with primer details shown in Supplemental Table S2. qPCR reactions contained Sensimix Sybr Rox (GC Biotech, Alphen aan den Rijn, the Netherlands) and primer mix and were run in a 384-well white opaque plate (Roche Diagnostics, Indianapolis, IN, USA) on a LightCycler 480 system (Roche Diagnostics). Melting curves were analyzed to verify specificity of the amplification, and relative quantity of the targets was assessed by LinRegPCR software (v2014.8; http://www.hartfaalcentrum.nl) (Heart Failure Research Centre, Amsterdam, the Netherlands). Study-specific reference genes (Supplemental Table S2) were used to calculate a GeNorm correction factor (https://genorm.cmgg.be/), which was used to normalize expression levels of the target genes.

\section{Immunohistochemistry}

OCT-embedded, frozen mouse gastrocnemius was cut $(7 \mu \mathrm{m})$, defrosted, and air dried at room temperature. Tissue was fixed with $4 \%$ paraformaldehyde and permeabilized with $0.2 \%$ Triton X-100 in PBS. Subsequently, samples were treated for 60 min with 3\% $\mathrm{H}_{2} \mathrm{O}_{2}$, blocked for 60 min in blocking solution [5\% (v/v) goat serum, 2\% (w/v) bovine serum albumin, 1:40 mouse-on-mouse blocking reagent (MOM; Vector Laboratories)] 


\section{CHAPTER 4}

at room temperature, and incubated overnight at $4^{\circ} \mathrm{C}$ with primary antibodies directed against laminin and paired box (PAX)-7 (1:250) (Supplemental Table S1). Samples were incubated in horseradish peroxidase-conjugated streptavidin and goat anti-rabbit 555 (1:200) for $60 \mathrm{~min}$ at room temperature, and subsequently for $7 \mathrm{~min}$ in tyramide working solution and reaction stop reagent, according to the manufacturer's protocol (Tyramide SuperBoost Kit; Thermo Fisher Scientific). Nuclei were stained with DAPI, after which cover slips were mounted with Mowiol (MilliporeSigma). After staining, images were digitally captured with fluorescence microscopy (Olympus IX81; Olympus, Hamburg, Germany) connected to a digital camera (EXi Aqua; QImaging, Surrey, BC, Canada) at x400 magnification. Image processing and quantitative analyses were performed with ImageJ (National Institutes of Health, Bethesda, MD, USA).

Laminin staining was used to determine the cell borders. Within each image, the mean fiber CSA, the number of myonuclei per fiber, and the mean fiber CSA per nucleus were quantified. Satellite cells (SCs) were manually counted and were defined as PAX7 ${ }^{+}$ nuclei located within the basal lamina.

\section{Data analysis and statistics}

To address the relation between alterations in skeletal muscle mass- and oxidative metabolism-related processes during RL after disuse, clustering of human skeletal muscle biochemical and physiologic parameters was performed with the SciPy (v.0.19.1) toolkit [41]. The RL response (RL-UL) per parameter was expressed as the percentage change from UL. The Spearman correlation coefficient between each pair of relative $\mathrm{RL}$ responses (i.e., $\mathrm{R}_{\mathrm{j}, \mathrm{k}}$ ) was computed and subsequently transformed to Euclidean distance $\left(D_{j, k}\right)$ with (Eq. 1):

$$
D_{j, k}=\sqrt{1-\left|R_{j, k}\right|}
$$

The resulting Euclidean distance matrix was hierarchically clustered based on the between-cluster average linkage using SciPy's cluster.hierarchy.linkage module and visualized using the clustermap module within Seaborn v.0.8.1 [42]. Differences in the distribution of markers between clusters were tested by Pearson's $X^{2}$ test with post-hoc analysis based on adjusted residuals with Bonferroni correction.

The $\mathrm{RL}$ response per parameter of paired data (human biopsies) was tested by pairedsamples t-test. The time-dependent reloading response (mouse muscle) was tested by 1-way ANOVA with Dunnett's post-hoc test, using UL as a reference group. Data are expressed as means \pm SEM, unless indicated otherwise. Statistical analyses were performed with SPSS Statistics (v.22.0; IBM, Armonk, NY, USA). 


\section{RESULTS}

One week of remobilization after leg immobilization-induced atrophy tended to increase quadriceps CSA (Table 1). However, neither quadriceps CSA nor muscle force measured by 1-RM had returned to preimmobilization baseline levels, indicating that recovery was still ongoing.

Table 1. | Response to immobilization

\begin{tabular}{llll}
\hline & Pre-immobilization & Post-immobilization & Remobilization \\
\hline Body weight (kg) & $70.9 \pm 3.3$ & $72.0 \pm 3.2$ & $71.7 \pm 3.1$ \\
Quadriceps CSA $\left(\mathbf{m m}^{2}\right)$ & $8004 \pm 320$ & $7562 \pm 303^{* * *}$ & $7671 \pm 315^{* * / *}$ \\
1-RM (kg) & $57 \pm 3$ & $50 \pm 2^{* *}$ & $52 \pm 2^{*}$ \\
\hline
\end{tabular}

Data are expressed as means $\pm S E M .{ }^{*} p<0.05,{ }^{* *} p<0.01,{ }^{* * *} p<0.001$ vs. preimmobilization, $\# p<0.1$ vs. postimmobilization.

\section{Regulators and mediators of myogenesis, autophagy, and mitochondrial mass and dynamics correlate during recovery from muscle disuse in humans}

To address whether there is a correlation between alterations in skeletal muscle massand oxidative metabolism-related processes during $\mathrm{RL}$ after disuse, we measured the protein and mRNA expression of several regulators and mediators of protein turnover, mitochondrial turnover, and myonuclear turnover, before and after $7 \mathrm{~d}$ of RL in humans and clustered the correlations between relative $\mathrm{RL}$ responses based on similarity. From this, 4 clusters of parameters were identified (Figure 1).

Cluster 1 appeared enriched in markers of protein turnover signaling, whereas cluster 2 seemed to be enriched in markers of muscle mass and function (Supplemental Table S3). Furthermore, two major clusters $(3,4)$ occurred in which markers of myogenesis and autophagy were strongly represented (Supplemental Table S3). This strong representation of myogenesis and autophagy markers was accompanied by a high percentage of markers of mitochondrial turnover and mitochondrial dynamics in cluster 3, whereas cluster 4 was concomitantly enriched in markers of mitochondrial mass and appeared enriched in markers of mitochondrial dynamics, and to some extent, protein turnover (Supplemental Table S3). Furthermore, most of the myogenesis markers represented in cluster 3 [i.e., myogenic factor (MYF)-5, myosin heavy chain (MYH)-8, myogenin (MYOG), and MYH9 mRNA] are associated with late stages of myogenesis (i.e., differentiation and fusion), whereas most of the myogenesis regulators and markers represented in cluster 4 [i.e., myostatin (MSTN) protein, and cyclin D1, PAX7, and cadherin 15 mRNA] are associated with early stages of myogenesis 


\section{CHAPTER 4}

(i.e., proliferation). Moreover, most of the autophagy markers in cluster 3 [i.e., optineurin (OPTN) mRNA, and microtubule associated protein 1 light chain 3 b (MAP1LC3B)-II, MAP1LC3B-I, and beclin (BECN)-1 protein] are indicators of autophagosome formation, whereas most of the autophagy markers in cluster 4 [i.e., phosphorylated unc-51 like autophagy activating kinase 1 (ULK1) ser556, pULK1ser556:ULK1 ratio, pULK1ser758, pULK1 ser758:ULK1 ratio, GABA type A receptor associated protein like 1 (GABARAPL1), and sequestosome 1 (SQSTM1) protein] are indicators of autophagy initiation.

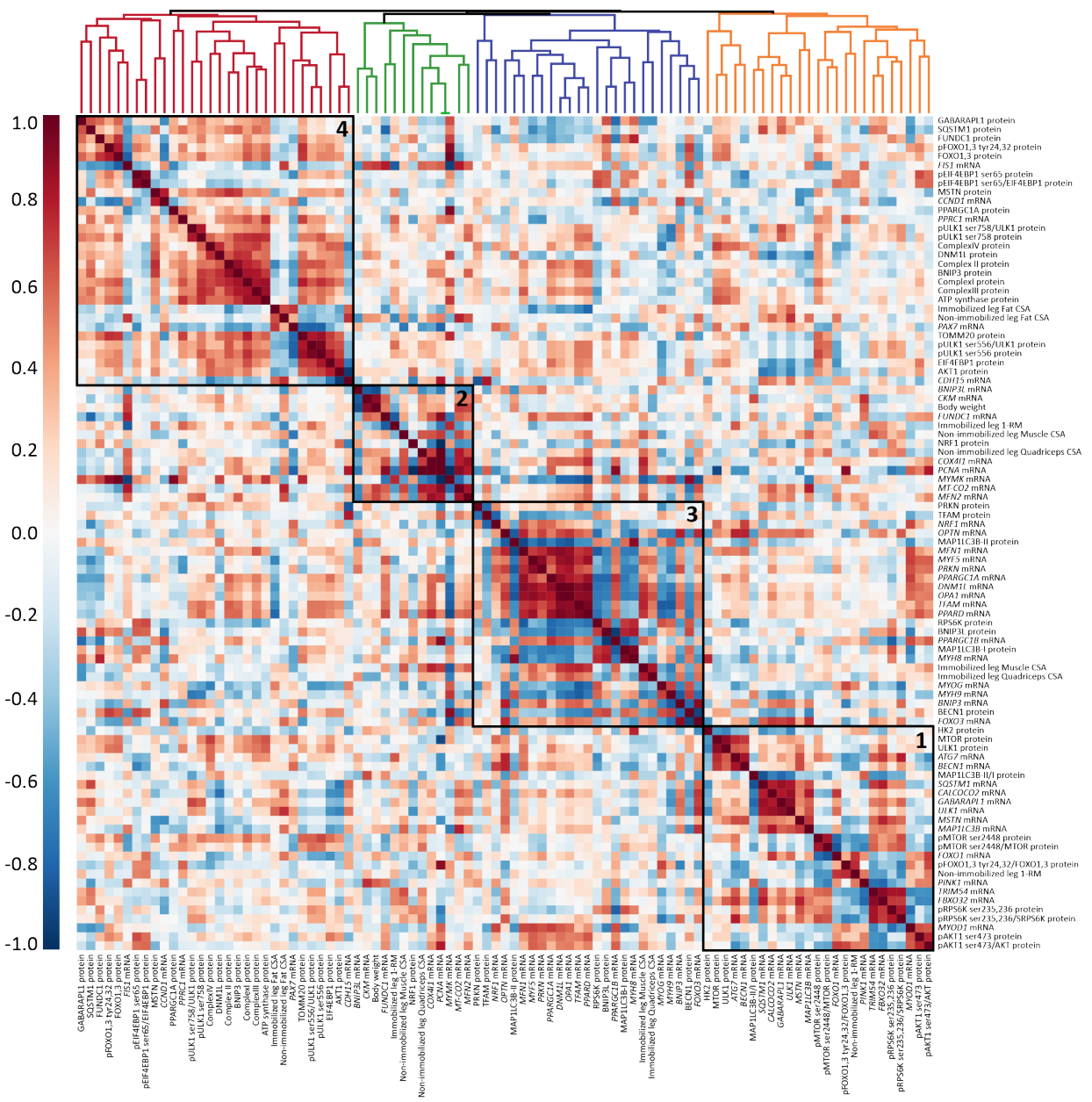

Figure 1. | A correlation heatmap and cluster dendrogram of biochemical and functional parameters during skeletal muscle recovery from disuse in humans. Each square represents the Spearman correlation coefficient between the relative $R L$ responses of parameters displayed on the $x$-and $y$-axis, with colors indicating the strength and direction of the correlation. 


\section{Induction of late myogenic markers after $5 \mathrm{~d}$ of $\mathrm{RL}$ in mice}

The distinct clustering of early (cluster 4) and late (cluster 3) myogenesis markers upon remobilization in human subjects may reflect a time-dependent expression pattern of these markers, as previously observed for early myogenesis markers by Pansters et al. [30]. This RL time-course mouse study was therefore used to address those time-dependent expression patterns. In this study, UL decreased the myofiber CSA, myonuclear domain, and number of nuclei per fiber (Figure $2 \mathrm{~A}-\mathrm{C}$ ). Ongoing recovery was indicated by an incomplete normalization of CSA and myonuclear domain up to RL-8, and the number of nuclei per fiber was significantly restored at RL-3 and -8 . This restoration of the myonuclei was paralleled by a tendency toward an increased number of SCs at RL-3 (Figure 2D-E).

A

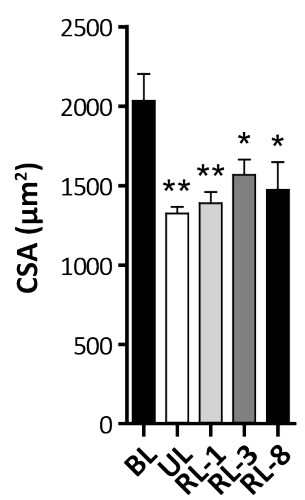

B

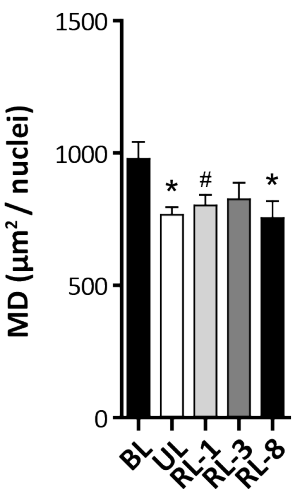

C

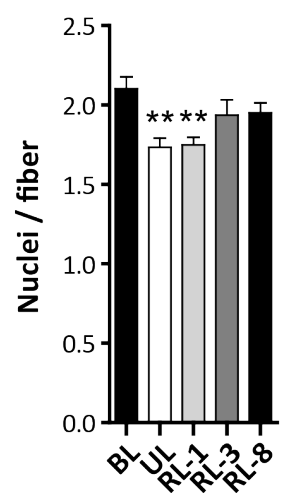

D

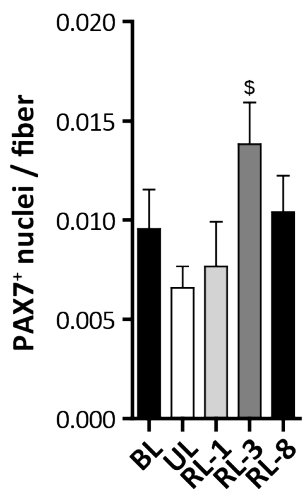

E

Laminin + DAPI

Laminin + PAX7
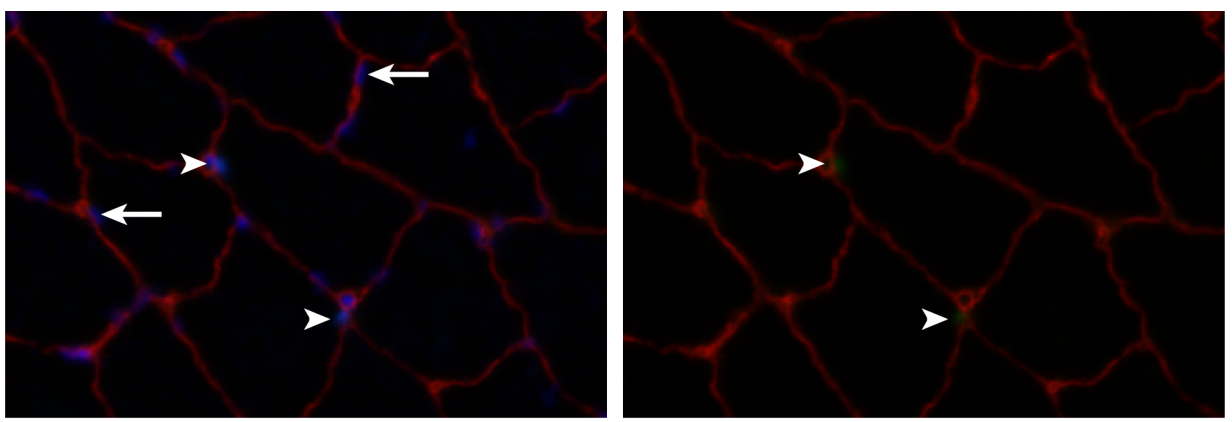

Figure 2. | Changes in myofiber CSA, myonuclear domain (MD), and the number of myonuclei and $S C s$ during muscle disuse and recovery in mice. A-D) Gastrocnemius muscles were collected at baseline (BL), after $14 d$ of $U L$, or after 1, 3, or $8 d$ of $R L$, for immunohistochemical determination of mean muscle fiber CSA (A); MD (B); mean number of myonuclei (C); and mean number of PAX7+ $S C S$ per muscle fiber (D). E) Representation of immunohistochemical detection of Laminin (red) and PAX7 (green), and nuclei stained with DAPI (blue). Arrowheads: $P A X 7^{+} S C$, arrows: myofiber nuclei. \#p $<0.1$, ${ }^{*} p<0.05,{ }^{* *} p<0.01, v s$. BL, and $\$ p<0.05$ vs. UL. 


\section{CHAPTER 4}

To assess whether late myogenesis markers indeed display a distinct temporal expression pattern, we measured markers of SC fusion and early myofiber regeneration in mouse gastrocnemius at 1, 2, 3, 5, and $8 \mathrm{~d}$ of RL after hindlimb suspension. At RL-1 and -2 , Myh9 was transiently increased and gradually normalized at RL-5 and -8 (Figure 3). The expression of another, novel fusion marker, myomaker (Mymk), was strongly but transiently induced at RL-5. Furthermore, Myh8 was induced at RL-5 and -8. Expression of the muscle-specific gene creatine kinase $(\mathrm{Ckm})$ was reduced upon $\mathrm{UL}$, and was transiently further reduced at RL-1, -2 and -3 , whereas expression of the negative regulator of muscle mass Mstn was reduced at RL-5 and -8.

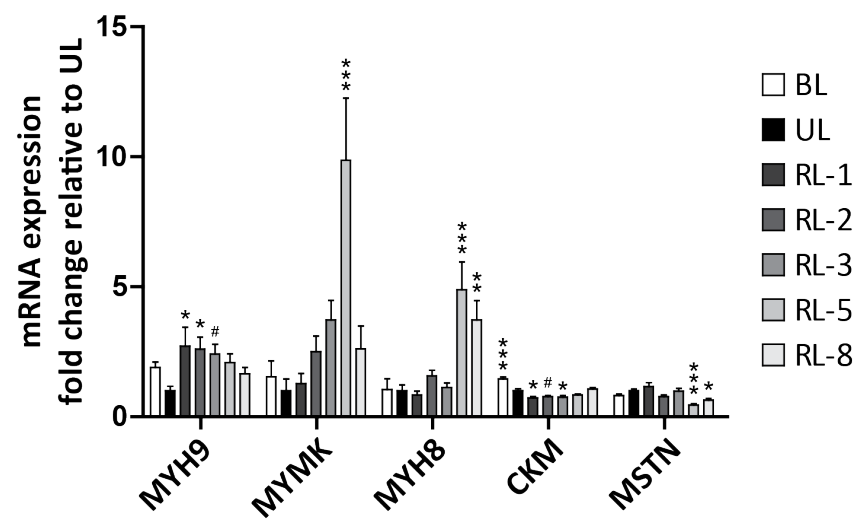

Figure 3. | Temporal changes in mRNA expression of markers and mediators of late myogenesis upon recovery from muscle disuse in mice. mRNA expression of $M Y H 9$, MYMK, MYH8, creatine kinase, and MSTN was assessed in gastrocnemius upon recovery from $14 d$ hindlimb suspension. $\# p<0.1$, ${ }^{*} p<0.05,{ }^{* *} p<0.01,{ }^{* * *} p<0.001$ vs. UL.

\section{Parallel modulation of autophagy and myogenesis in mice}

To address whether the temporal expression patterns of early and late myogenesis markers coincide with a temporal regulation of autophagy (as suggested from clusters 3 and 4), mRNA and protein levels of indicators of autophagosome formation and markers of autophagy capacity and autophagy initiation were measured.

The relative protein expression of Map1lc3b-I displayed an acute but transient increase at RL-1, -2 and -3, whereas Map1lc3b-II levels remained largely unaltered (Figure 4). The Map1lc3b-II:I ratio was decreased at RL-1, but subsequently normalized, and even increased at RL-8. Furthermore, the relative mRNA expression of Map1lc3b was decreased at RL-2, -3 and -5 , and seemed to be partially normalized at RL-8 (Figure 5). In contrast, Gabarapl1 and Sqstm1 protein and mRNA expression displayed an acute but transient increase at RL-1 compared to UL, although Gabarapl1 mRNA was initially reduced upon UL (Figure 4, Figure 5). The mRNA expression of Optn, Becn 1 and autophagy related-7 were unaltered during $\mathrm{RL}$ (Figure 5). 


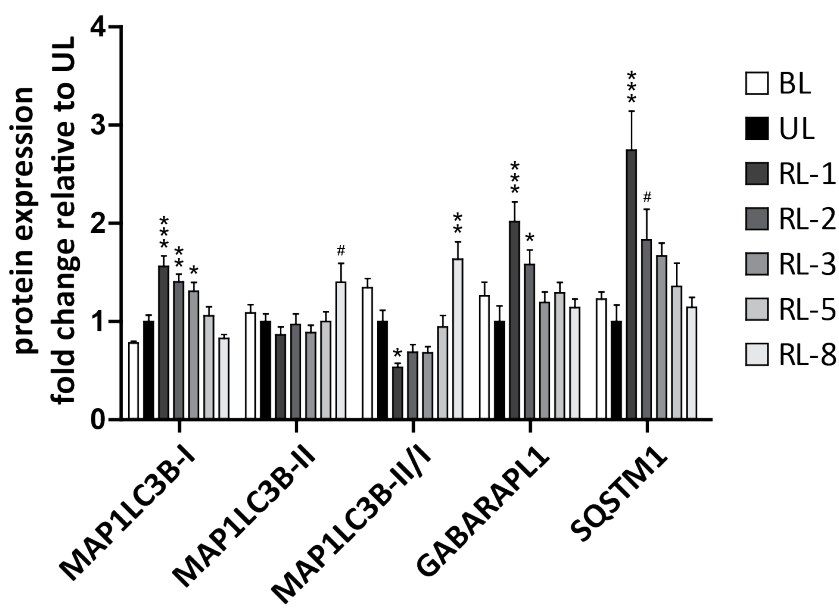

Figure 4. | Temporal changes in protein expression of autophagosome formation indicators upon recovery from muscle disuse in mice. Protein expression of MAP1LC3B-I, MAP1LC3B-II, MAP1LC3BII:I, GABARAPL 1, and SQSTM1 was assessed in gastrocnemius upon recovery from $14 d$ hindlimb suspension. $\# p<0.1,{ }^{*} p<0.05,{ }^{* *} p<0.01,{ }^{* * *} p<0.001$ vs. UL.

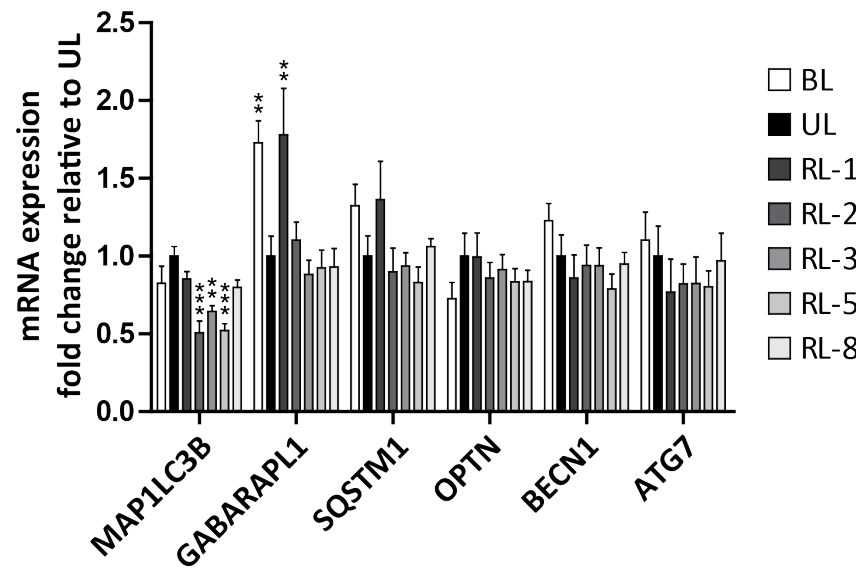

Figure 5.|Temporal changes in MRNA expression of autophagy-related genes upon recovery from muscle disuse in mice. MRNA expression of MAP1LC3B, GABARAPL1, SQSTM1, OPTN, BECN1, and autophagy related (ATG)-7 was assessed in gastrocnemius upon recovery from $14 d$ hindlimb suspension. ${ }^{* *} p<0.01,{ }^{* * *} p<0.001$ vs. UL. 


\section{CHAPTER 4}

To further address a main regulatory step in the conversion of changes in autophagy capacity into flux, the activating and inhibiting phosphorylation of Ulk1 was assessed. Both absolute and relative activating Ulk1 phosphorylation (ser555) were increased during RL, up to RL-3 (Figure 6). However, in parallel, both absolute and relative inhibiting Ulk1 phosphorylation (ser757) were increased during RL, up to RL-5.

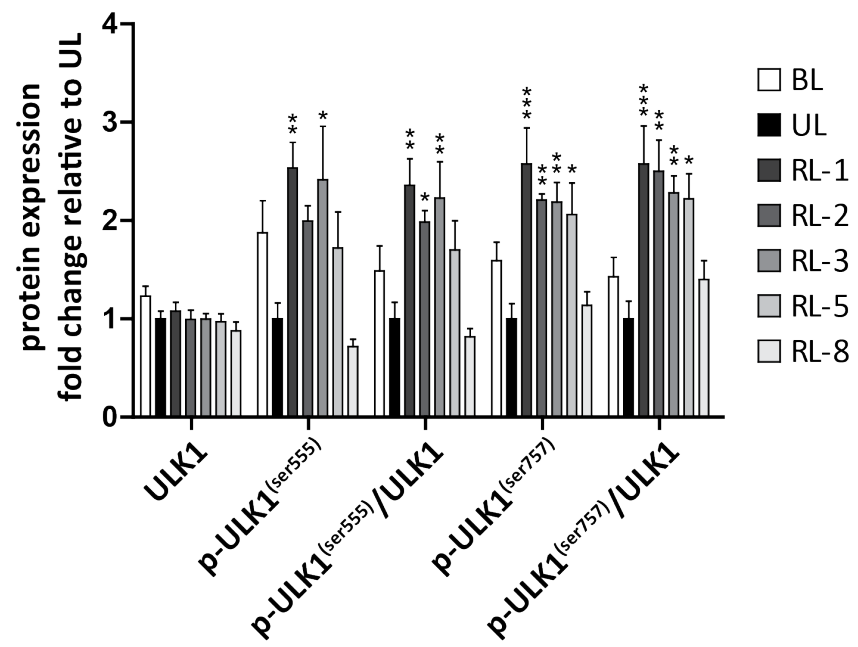

Figure 6. | Temporal changes in protein expression of markers of autophagy initiation upon recovery from muscle disuse in mice. Protein expression of ULK1, p-ULK1(ser555), p-ULK1(ser555):ULK1 ratio, $p$-ULK1(ser757), and p-ULK1(ser757):ULK1 ratio was assessed in gastrocnemius upon recovery from $14 d$ hindlimb suspension. ${ }^{*} p<0.05,{ }^{* *} p<0.01,{ }^{* * *} p<0.001$ vs. UL.

\section{Temporal induction of mitophagy during recovery from disuse in mice}

Next to the predominance of markers of autophagosome formation in cluster 3, this cluster also appeared to be enriched in markers of mitophagy. We therefore assessed the temporal expression of mitophagy markers during recovery from disuse in mice.

No significant alterations in protein expression of BCL2 interacting protein 3 like (Bnip3l) or parkin RBR E3 ubiquitin protein ligase (Prkn) were observed during recovery after disuse. However, the protein expression of Bnip3 increased at RL-1, subsequently returned to UL values, and decreased at RL-5 and -8 (Figure 7). Furthermore, FUN14 domain containing 1 (Fundc1) protein expression was increased at RL-8 compared to UL, although an initial decrease was observed upon UL. Corresponding to its change in protein abundance, Fundc1 mRNA expression was increased at RL-8 (Figure 8). Furthermore, Bnip3I mRNA expression was decreased, and phosphatase and tensin homolog (PTEN)-induced putative kinase 1 (Pink)-1 tended to be decreased upon remobilization, up to $\mathrm{d} R \mathrm{RL}-8$, but no changes in the expression of Prkn mRNA were observed. 


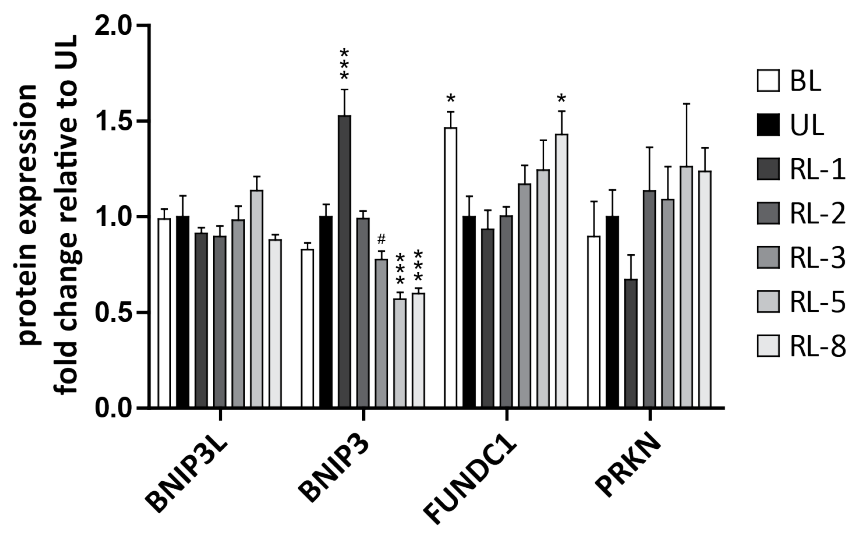

Figure 7.| Temporal changes in protein expression of mitophagy regulators and mediators upon recovery from muscle disuse in mice. Protein expression of BNIP3L, BNIP3, FUNDC1, and PRKN was assessed in gastrocnemius upon recovery from $14 d$ hindlimb suspension. $\# p<0.1,{ }^{*} p<0.05$, ${ }^{* * *} p<0.001$ vs. UL.

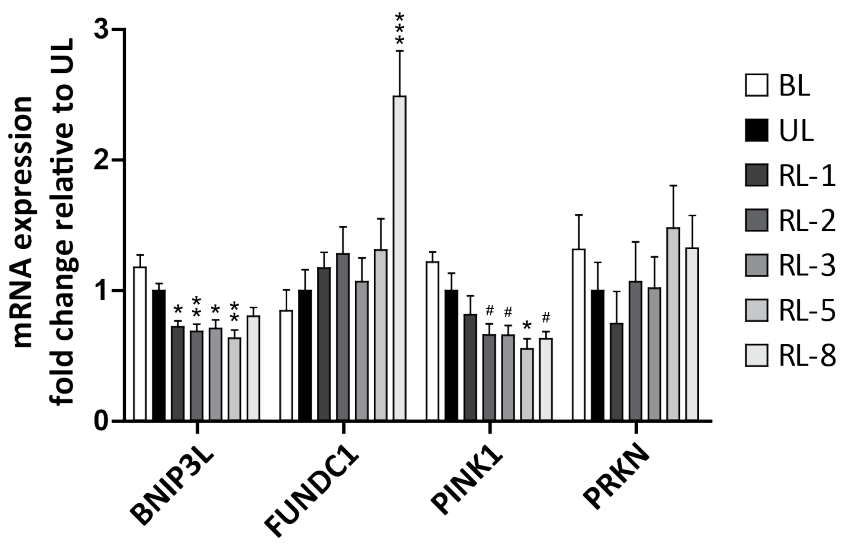

Figure 8. | Temporal changes in mRNA expression of mitophagy-related genes upon recovery from muscle disuse in mice. MRNA expression of BNIP3L, BNIP3, FUNDC1, PINK1, and PRKN was assessed in gastrocnemius upon recovery from $14 d$ hindlimb suspension. $\# p<0.1,{ }^{*} p<0.05,{ }^{* *} p<0.01,{ }^{* * * *} p<0.001$ vs. UL. 


\section{CHAPTER 4}

\section{DISCUSSION}

Alterations in the regulation of both muscle mass and metabolic plasticity during recovery from disuse have been reported, but the interaction between these processes is understudied. Hence, we explored this potential interaction by measuring established molecular regulators and mediators of processes that determine muscle mass and metabolism in human and mouse muscle and showed that molecular markers of myogenesis, autophagy, and mitochondrial mass and dynamics change coordinately during recovery from disuse.

In the current human study, 1 wk of remobilization after leg immobilization-induced atrophy increased quadriceps CSA compared to postimmobilization, but did not return quadriceps CSA or muscle force measured by 1-RM to preimmobilization baseline levels. This finding is in line with those in studies that showed an incomplete normalization of CSA [43, 44] and muscle strength [44] upon 1 wk of recovery after immobilization and a complete normalization of muscle mass and strength after 2 wk of recovery $[25,44-47]$. This result shows that after $1 \mathrm{wk}$ of remobilization, skeletal muscle recovery is still ongoing, and thus allows us to study the processes that are associated with recovery.

We detected substantial interindividual variation in the human molecular responses to RL (Supplemental Figure S1), which was not affected by correction for creatine supplementation. We exploited this variation to assess the relation between molecular $\mathrm{RL}$ responses. Based on hierarchical cluster analysis of the correlations between relative $\mathrm{RL}$ responses, we identified 4 clusters of parameters. The coappearance of markers and mediators of classic muscle mass- and oxidative metabolism-related processes within clusters 3 and 4 may point at an interdependency or joint regulation of these processes during recovery from atrophy. Although we cannot exclude creatine supplementation as a potential confounding factor and a modulatory effect of creatine supplementation on certain molecular processes has been suggested [48, 49], there is no indication of an effect of creatine supplementation on the interrelationship between the regulatory processes associated with recovery from muscle atrophy, and correction for creatine supplementation does not alter the pattern of correlations between RL effects. Markers that regulate and mediate different stages of myogenesis and autophagy displayed a distinct clustering, which may be related to differences in their temporal expression patterns. Between-subject asynchronicity in such temporal expression patterns may contribute to the observed variation, which - despite its applicability in the assessment of between-variable associations — limits the statistical 
power to detect relevant changes on a group level. Furthermore, interindividual asynchronicity, combined with a single time-point observation during recovery in humans, complicates the synthesis of a univocal conclusion on the molecular RL response. The high-resolution time-course study in mice was thus instrumental in the indepth exploration of the time-dependent molecular regulation of muscle mass and metabolic plasticity during disuse-induced atrophy recovery.

Similar to the study in humans, mice displayed an incomplete normalization of muscle weight [30] and muscle fiber CSA, which supports ongoing recovery over $8 \mathrm{~d}$ of RL. Three days of RL after hindlimb suspension transiently increased the number of PAX7+ SCs per fiber. This finding is in line with previous studies assessing SC numbers after relative short-term remobilization in rodents [50-52] and humans [46] and may explain the unaltered number of SCs upon longer term remobilization [53]. In accordance with the early induction of SC proliferation, our lab showed in another study an early and potentially biphasic induction of markers and mediators of the initial steps in myogenesis in this model [30]. In the current work, we showed for the first time that, subsequent to this increase in early myogenesis markers, the markers and mediators of late stages of myogenesis Mymk and Myh8 are increased toward the end of the $8 \mathrm{~d}$ RL phase. Furthermore, in line with previous studies [54-56], there was a partial restoration of the number of nuclei per fiber after $3 \mathrm{~d}$ of $\mathrm{RL}$, which supports the notion that SCs functionally contribute to skeletal muscle recovery after disuse [57]. Indeed, because the myonuclear domain was unaltered during remobilization, the increase in myonuclear content is expected to result in an increase in muscle fiber CSA. However, as this change in fiber CSA is not yet significantly detectable, we conclude that muscle fiber CSA is incompletely restored up to $8 \mathrm{~d}$ of remobilization. These findings could be speculated to result from a lag time between myonuclear accretion and a functional contribution of the newly incorporated nucleus to protein synthesis or to reflect a role for myonuclear accretion in muscle fiber metabolic plasticity. Despite the incomplete normalization of muscle fiber CSA and myonuclear domain over the time course examined in this mouse study, their recovery seemed to start as early as RL1. This is in agreement with the previously observed acute and sustained increase in protein synthesis signaling $[30,58-60]$ and increased protein synthesis rates upon $\mathrm{RL}$ [61]. This anabolic state precedes myonuclear accretion, which supports the notion that hypertrophy can occur to some extent independent of SCs [62]. Furthermore, previous studies have consistently shown a suppression of mediators of the UPS during recovery from disuse [27, 30, 44, 52, 59, 60, 63-65], whereas a transient increase in proteasome activity [27, 61] and ubiquitin-conjugated proteins [60] have been observed simultaneously. 


\section{CHAPTER 4}

In this study, we conducted a comprehensive analysis of markers of autophagosome formation, autophagy capacity, and autophagy initiation. From this, we conclude that autophagy is actively regulated both acutely, as well as after 5 and $8 \mathrm{~d}$ of RL, which appears to parallel the temporal regulation of myonuclear accretion. We showed an acute but transient increase in the autophagosome formation markers Map1lc3b-l, Gabarapl1 and Sqstm1 protein, and a decrease and subsequent increase in the Map1lc3b-II:I ratio that parallels the regulation of late stages of myogenesis. In line with our data, previous studies showed an increase in Sqstm 1 protein expression [27] and an increase in Map1lc3b-II:I ratio [31]. In addition, White et al. [63] reported an unaltered Map1lc3b-Il:I ratio after $14 \mathrm{~d}$ of $\mathrm{RL}$, potentially reflecting a normalization of autophagy signaling upon completed recovery. Furthermore, in line with previous studies $[59,66]$, we showed a transient decrease in the mRNA expression of the autophagy capacity indicator Map1lc3b and a transient increase in the mRNA expression of Gabarapl1 relative to UL. In addition to the reported increase in Ulk1 inhibiting phosphorylation $[27,29]$, we report a concomitant induction of Ulk1 inhibiting and activating phosphorylation. This temporal regulation of autophagy initiation resembles the expression pattern of early myogenesis markers observed by Pansters et al. [30].

Despite our comprehensive analysis of autophagy regulation, we did not measure autophagy flux and therefore cannot draw an unambiguous conclusion regarding the net effect of RL on the autophagy flux itself. Furthermore, alterations in expression levels of several autophagy regulators and mediators seem contradictory, which emphasizes the complexity of autophagy regulation during recovery after disuse. We speculate that this complexity reflects the degradation of specific targets (e.g. mitochondria), which is in accordance with a possible involvement of autophagy in skeletal muscle metabolic plasticity in addition to its classic role in muscle mass regulation $[67,68]$. Recent papers suggest a crucial role for autophagy, and more specifically, mitophagy, in skeletal muscle functional regeneration $[69,70]$.

During recovery after disuse in mice, the gene expression of the mitochondrial damagerelated mitophagy regulator Pink1 tends to be decreased, whereas Prkn is unaltered. In addition, Prkn protein expression is unaltered during remobilization, although it should be noted that this mitophagy pathway is also modulated by posttranslational modifications [71], which were not assessed in this study. Receptor-mediated mitophagy via BNIP3L, BNIP3, and FUNDC1 can be induced by both hypoxia and increased oxidative stress [72], of which an increase during RL has been documented $[73,74]$. Bnip3 protein expression transiently increased, and subsequently decreased during recovery, which parallels the previously reported alterations in Bnip3 mRNA 
expression in this mouse study [30], and the increase in Bnip3 protein expression reported by Kang and Ji [74]. In contrast, Bnip3l protein expression is unaltered upon recovery after disuse, whereas Bnip3I mRNA expression displays a persistent decrease up to RL-5. The mRNA and protein expression of Fundc 1 is specifically induced at RL-8, suggesting a late-phase response.

These molecular alterations point toward a temporal induction of mitophagy both acutely and after $8 \mathrm{~d}$ of recovery after disuse. Nevertheless, previous data suggest a normalization of mitochondrial content upon several days of RL [34, 73-75], likely driven by an increase in mitochondrial biogenesis $[34,73]$. The induction of mitophagy in the presence of a recovery of mitochondrial content suggests the induction of mitochondrial remodeling during remobilization. Indeed, an increase in mitochondrial remodeling [75] and mitochondrial dynamics [34, 75] has been observed during recovery from disuse. This RL-induced mitochondrial remodeling may be a response to increased oxphos [76] and could serve to meet the altered metabolic demands during remobilization. Another observation is that a late increase in Fundc1 expression paralleled the temporal regulation of late-stage myogenesis markers. It is becoming increasingly clear that mitophagy [77], mitochondrial dynamics [78, 79], and metabolic reprogramming [80-83] are crucial for myogenic differentiation. Furthermore, Mishra et al. [76] showed that mitochondrial proteins compartmentalize to discrete domains located around a nucleus of origin that originates from SC-mediated myonuclear accretion. In line, myogenesis seems to be involved in the aerobic adaptation in response to endurance-type exercise [84]. Clearly, this indicates a close relation between muscle mass- and oxidative metabolism-related processes.

\section{Conclusion}

We showed, through the combination of a cluster analysis and high-resolution time-course study, that the molecular regulation of protein turnover, mitochondrial turnover, and myonuclear turnover are correlated and temporally associated during recovery from atrophy. This result suggests an interdependency or joint regulation of these muscle mass- and oxidative metabolism-related processes during recovery from atrophy and emphasizes the need to study these processes by integrated molecular analyses. 


\section{CHAPTER 4}

\section{REFERENCES}

1. Egan B, Zierath JR. Exercise metabolism and the molecular regulation of skeletal muscle adaptation. Cell Metab 2013;17(2):162-84.

2. Wall BT, van Loon LJ. Nutritional strategies to attenuate muscle disuse atrophy. Nutr Rev 2013;71(4):195-208.

3. Wall BT, Dirks ML, Snijders T, et al. Substantial skeletal muscle loss occurs during only 5 days of disuse. Acta Physiol (Oxf) 2014;210(3):600-11.

4. Brooks NE, Myburgh KH. Skeletal muscle wasting with disuse atrophy is multi-dimensional: the response and interaction of myonuclei, satellite cells and signaling pathways. Frontiers in physiology 2014;5:99.

5. Bierbrauer J, Koch S, Olbricht $C$, et al. Early type II fiber atrophy in intensive care unit patients with nonexcitable muscle membrane. Crit Care Med 2012;40(2):647-50.

6. Pette D, Staron RS. Transitions of muscle fiber phenotypic profiles. Histochem Cell Biol 2001;115(5):359-72.

7. $\mathrm{Yu} Z \mathrm{~B}$, Gao F, Feng $\mathrm{HZ}$, et al. Differential regulation of myofilament protein isoforms underlying the contractility changes in skeletal muscle unloading. Am J Physiol Cell Physiol 2007;292(3):C1192-203.

8. Stein TP, Wade CE. Metabolic consequences of muscle disuse atrophy. J Nutr 2005; 135(7):1824S-28S.

9. Gram M, Dahl R, Dela F. Physical inactivity and muscle oxidative capacity in humans. Eur J Sport Sci 2014;14(4):376-83.

10. Dirks ML, Wall BT, van de Valk B, et al. One Week of Bed Rest Leads to Substantial Muscle Atrophy and Induces Whole-Body Insulin Resistance in the Absence of Skeletal Muscle Lipid Accumulation. Diabetes 2016;65(10):2862-75.

11. Gram M, Vigelso A, Yokota $\mathrm{T}$, et al. Skeletal muscle mitochondrial $\mathrm{H} 2 \mathrm{O} 2$ emission increases with immobilization and decreases after aerobic training in young and older men. J Physiol 2015;593(17):4011-27.

12. Romanello V, Guadagnin $\mathrm{E}$, Gomes $\mathrm{L}$, et al. Mitochondrial fission and remodelling contributes to muscle atrophy. EMBO J 2010;29(10):1774-85.

13. Seene T, Kaasik P, Riso EM. Review on aging, unloading and reloading: changes in skeletal muscle quantity and quality. Arch Gerontol Geriatr 2012;54(2):374-80.

14. Rizzoli R, Reginster JY, Arnal JF, et al. Quality of life in sarcopenia and frailty. Calcif Tissue Int 2013;93(2):101-20.

15. Schols AM, Broekhuizen R, Weling-Scheepers CA, et al. Body composition and mortality in chronic obstructive pulmonary disease. Am J Clin Nutr 2005;82(1):53-9.

16. Martin L, Birdsell L, Macdonald N, et al. Cancer cachexia in the age of obesity: skeletal muscle depletion is a powerful prognostic factor, independent of body mass index. J Clin Oncol 2013;31(12):1539-47.

17. Marzetti E, Calvani R, Tosato $M$, et al. Sarcopenia: an overview. Aging Clin Exp Res 2017;29(1):11-17.

18. Dirks ML, Wall BT, Snijders T, et al. Neuromuscular electrical stimulation prevents muscle disuse atrophy during leg immobilization in humans. Acta Physiol (Oxf) 2014;210(3):628-41.

19. Dirks ML, Wall BT, Nilwik R, et al. Skeletal muscle disuse atrophy is not attenuated by dietary protein supplementation in healthy older men. J Nutr 2014;144(8):1196-203.

20. Ferrando AA, Paddon-Jones $D$, Hays NP, et al. EAA supplementation to increase nitrogen intake improves muscle function during bed rest in the elderly. Clin Nutr 2010;29(1):18-23.

21. Paddon-Jones D, Sheffield-Moore M, Urban RJ, et al. Essential amino acid and carbohydrate 
supplementation ameliorates muscle protein loss in humans during 28 days bedrest. J Clin Endocrinol Metab 2004;89(9):4351-8.

22. Reidy PT, McKenzie Al, Brunker P, et al. Neuromuscular Electrical Stimulation Combined with Protein Ingestion Preserves Thigh Muscle Mass But Not Muscle Function in Healthy Older Adults During 5 Days of Bed Rest. Rejuvenation Res 2017

23. Dideriksen $\mathrm{K}$, Boesen AP, Kristiansen JF, et al. Skeletal muscle adaptation to immobilization and subsequent retraining in elderly men: No effect of anti-inflammatory medication. Exp Gerontol 2016;82:8-18.

24. Tanner RE, Brunker LB, Agergaard J, et al. Age-related differences in lean mass, protein synthesis and skeletal muscle markers of proteolysis after bed rest and exercise rehabilitation. J Physiol 2015;593(18):4259-73.

25. Suetta C, Hvid LG, Justesen L, et al. Effects of aging on human skeletal muscle after immobilization and retraining. Journal of applied physiology 2009;107(4):1172-80.

26. Joanisse S, Nederveen JP, Snijders T, et al. Skeletal Muscle Regeneration, Repair and Remodelling in Aging: The Importance of Muscle Stem Cells and Vascularization. Gerontology 2017;63(1):91-100.

27. Baehr LM, West DW, Marcotte G, et al. Age-related deficits in skeletal muscle recovery following disuse are associated with neuromuscular junction instability and ER stress, not impaired protein synthesis. Aging (Albany NY) 2016;8(1):127-46.

28. Theriault ME, Pare ME, Lemire BB, et al. Regenerative defect in vastus lateralis muscle of patients with chronic obstructive pulmonary disease. Respir Res 2014;15:35.

29. Kelleher AR, Pereira SL, Jefferson LS, et al. REDD2 expression in rat skeletal muscle correlates with nutrient-induced activation of mTORC1: responses to aging, immobilization, and remobilization. Am J Physiol Endocrinol Metab 2015;308(2):E122-9.

30. Pansters NA, Schols AM, Verhees KJ, et al. Muscle-specific GSK-3beta ablation accelerates regeneration of disuse-atrophied skeletal muscle. Biochim Biophys Acta 2015;1852(3):490506.

31. Takahashi H, Suzuki Y, Mohamed JS, et al. Epigallocatechin-3-gallate increases autophagy signaling in resting and unloaded plantaris muscles but selectively suppresses autophagy protein abundance in reloaded muscles of aged rats. Exp Gerontol 2017;92:56-66.

32. Kang C, Yeo D, Ji LL. Muscle immobilization activates mitophagy and disrupts mitochondrial dynamics in mice. Acta Physiol (Oxf) 2016;218(3):188-97.

33. McClung JM, Davis JM, Wilson MA, et al. Estrogen status and skeletal muscle recovery from disuse atrophy. Journal of applied physiology 2006;100(6):2012-23.

34. Remels AH, Pansters NA, Gosker HR, et al. Activation of alternative NF-kappaB signaling during recovery of disuse-induced loss of muscle oxidative phenotype. Am J Physiol Endocrinol Metab 2014;306(6):E615-26.

35. Lopez-Lluch G. Essential role of mitochondrial dynamics in muscle physiology. Acta Physiol (Oxf) 2017;219(1):20-21.

36. Backx EM, Hangelbroek R, Snijders T, et al. Creatine Loading Does Not Preserve Muscle Mass or Strength During Leg Immobilization in Healthy, Young Males: A Randomized Controlled Trial. Sports Med 2017

37. Bodine SC, Latres E, Baumhueter $S$, et al. Identification of ubiquitin ligases required for skeletal muscle atrophy. Science 2001;294(5547):1704-8.

38. Ferreira R, Neuparth MJ, Ascensao A, et al. Skeletal muscle atrophy increases cell proliferation in mice gastrocnemius during the first week of hindlimb suspension. Eur J Appl Physiol 2006;97(3):340-6.

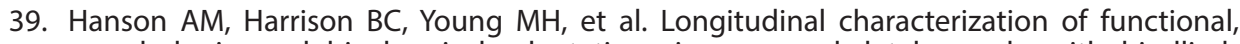
morphologic, and biochemical adaptations in mouse skeletal muscle with hindlimb suspension. Muscle Nerve 2013;48(3):393-402. 


\section{CHAPTER 4}

40. Ruijter JM, Thygesen $\mathrm{HH}$, Schoneveld OJ, et al. Factor correction as a tool to eliminate between-session variation in replicate experiments: application to molecular biology and retrovirology. Retrovirology 2006;3:2.

41. Oliphant TE. Python for scientific computing. Comput Sci Eng 2007;9(3):10-20.

42. mwaskom/seaborn: v0.8.1 (September 2017) [program]. September 3 version. https://doi. org/10.5281/zenodo.883859, 2017.

43. Hvid LG, Suetta C, Nielsen JH, et al. Aging impairs the recovery in mechanical muscle function following 4 days of disuse. Exp Gerontol 2014;52:1-8.

44. Jones SW, Hill RJ, Krasney PA, et al. Disuse atrophy and exercise rehabilitation in humans profoundly affects the expression of genes associated with the regulation of skeletal muscle mass. FASEB journal : official publication of the Federation of American Societies for Experimental Biology 2004;18(9):1025-7.

45. Hortobagyi T, Dempsey L, Fraser D, et al. Changes in muscle strength, muscle fibre size and myofibrillar gene expression after immobilization and retraining in humans. J Physiol 2000;524 Pt 1:293-304.

46. Suetta C, Frandsen $U$, Mackey AL, et al. Ageing is associated with diminished muscle regrowth and myogenic precursor cell expansion early after immobility-induced atrophy in human skeletal muscle. J Physiol 2013;591(15):3789-804.

47. Hespel P, Op't Eijnde B, Van Leemputte M, et al. Oral creatine supplementation facilitates the rehabilitation of disuse atrophy and alters the expression of muscle myogenic factors in humans. J Physiol 2001;536(Pt 2):625-33.

48. Kim J, Lee J, Kim S, et al. Role of creatine supplementation in exercise-induced muscle damage: A mini review. J Exerc Rehabil 2015;11(5):244-50.

49. Deldicque L, Atherton $P$, Patel $R$, et al. Effects of resistance exercise with and without creatine supplementation on gene expression and cell signaling in human skeletal muscle. Journal of applied physiology 2008;104(2):371-8.

50. Stevens-Lapsley JE, Ye F, Liu M, et al. Impact of viral-mediated IGF-I gene transfer on skeletal muscle following cast immobilization. Am J Physiol Endocrinol Metab 2010;299(5):E730-40.

51. Ye F, Mathur S, Liu M, et al. Overexpression of insulin-like growth factor-1 attenuates skeletal muscle damage and accelerates muscle regeneration and functional recovery after disuse. Exp Physiol 2013;98(5):1038-52.

52. Park $S$, Brisson BK, Liu M, et al. Mature IGF-I excels in promoting functional muscle recovery from disuse atrophy compared with pro-IGF-IA. Journal of applied physiology 2014;116(7):797-806.

53. Jackson JR, Mula J, Kirby TJ, et al. Satellite cell depletion does not inhibit adult skeletal muscle regrowth following unloading-induced atrophy. Am J Physiol Cell Physiol 2012;303(8):C85461.

54. Mozdziak PE, Pulvermacher PM, Schultz E. Muscle regeneration during hindlimb unloading results in a reduction in muscle size after reloading. Journal of applied physiology 2001;91(1):183-90.

55. Wang XD, Kawano F, Matsuoka Y, et al. Mechanical load-dependent regulation of satellite cell and fiber size in rat soleus muscle. Am J Physiol Cell Physiol 2006;290(4):C981-9.

56. Mitchell PO, Pavlath GK. Skeletal muscle atrophy leads to loss and dysfunction of muscle precursor cells. Am J Physiol Cell Physiol 2004;287(6):C1753-62.

57. Mitchell PO, Pavlath GK. A muscle precursor cell-dependent pathway contributes to muscle growth after atrophy. Am J Physiol Cell Physiol 2001;281(5):C1706-15.

58. Washington TA, White JP, Davis JM, et al. Skeletal muscle mass recovery from atrophy in IL-6 knockout mice. Acta Physiol (Oxf) 2011;202(4):657-69.

59. Smith HK, Matthews KG, Oldham JM, et al. Translational signalling, atrogenic and myogenic 
gene expression during unloading and reloading of skeletal muscle in myostatin-deficient mice. PLoS One 2014;9(4):e94356.

60. Kramerova I, Kudryashova E, Venkatraman G, et al. Calpain 3 participates in sarcomere remodeling by acting upstream of the ubiquitin-proteasome pathway. Hum Mol Genet 2005;14(15):2125-34.

61. Lang SM, Kazi AA, Hong-Brown L, et al. Delayed recovery of skeletal muscle mass following hindlimb immobilization in mTOR heterozygous mice. PLoS One 2012;7(6):e38910.

62. McCarthy JJ, Mula J, Miyazaki M, et al. Effective fiber hypertrophy in satellite cell-depleted skeletal muscle. Development 2011;138(17):3657-66.

63. White JR, Confides AL, Moore-Reed S, et al. Regrowth after skeletal muscle atrophy is impaired in aged rats, despite similar responses in signaling pathways. Exp Gerontol 2015;64:17-32.

64. Andrianjafiniony T, Dupre-Aucouturier S, Letexier D, et al. Oxidative stress, apoptosis, and proteolysis in skeletal muscle repair after unloading. Am J Physiol Cell Physiol 2010;299(2):C307-15.

65. Chacon-Cabrera A, Lund-Palau H, Gea J, et al. Time-Course of Muscle Mass Loss, Damage, and Proteolysis in Gastrocnemius following Unloading and Reloading: Implications in Chronic Diseases. PLoS One 2016;11(10):e0164951.

66. Slimani L, Micol D, Amat J, et al. The worsening of tibialis anterior muscle atrophy during recovery post-immobilization correlates with enhanced connective tissue area, proteolysis, and apoptosis. Am J Physiol Endocrinol Metab 2012;303(11):E1335-47.

67. Ferraro E, Giammarioli AM, Chiandotto S, et al. Exercise-induced skeletal muscle remodeling and metabolic adaptation: redox signaling and role of autophagy. Antioxid Redox Signal 2014;21(1):154-76.

68. Kaur J, Debnath J. Autophagy at the crossroads of catabolism and anabolism. Nat Rev Mol Cell Biol 2015;16(8):461-72.

69. Call JA, Wilson RJ, Laker RC, et al. Ulk1-mediated autophagy plays an essential role in mitochondrial remodeling and functional regeneration of skeletal muscle. Am J Physiol Cell Physiol 2017;312(6):C724-C32.

70. Nichenko AS, Southern WM, Atuan M, et al. Mitochondrial maintenance via autophagy contributes to functional skeletal muscle regeneration and remodeling. Am J Physiol Cell Physiol 2016;311(2):C190-200.

71. Durcan TM, Fon EA. The three 'P's of mitophagy: PARKIN, PINK1, and post-translational modifications. Genes \& development 2015;29(10):989-99.

72. Wu H, Chen Q. Hypoxia activation of mitophagy and its role in disease pathogenesis. Antioxid Redox Signal 2015;22(12):1032-46.

73. Kang C, Goodman CA, Hornberger TA, et al. PGC-1alpha overexpression by in vivo transfection attenuates mitochondrial deterioration of skeletal muscle caused by immobilization. FASEB journal : official publication of the Federation of American Societies for Experimental Biology 2015;29(10):4092-106.

74. Kang C, Ji LL. PGC-1alpha overexpression via local transfection attenuates mitophagy pathway in muscle disuse atrophy. Free Radic Biol Med 2016;93:32-40.

75. Liu J, Peng Y, Feng Z, et al. Reloading functionally ameliorates disuse-induced muscle atrophy by reversing mitochondrial dysfunction, and similar benefits are gained by administering a combination of mitochondrial nutrients. Free Radic Biol Med 2014;69:116-28.

76. Mishra P, Varuzhanyan G, Pham AH, et al. Mitochondrial Dynamics is a Distinguishing Feature of Skeletal Muscle Fiber Types and Regulates Organellar Compartmentalization. Cell Metab 2015;22(6):1033-44.

77. Sin J, Andres AM, Taylor DJ, et al. Mitophagy is required for mitochondrial biogenesis and myogenic differentiation of C2C12 myoblasts. Autophagy 2016;12(2):369-80. 


\section{CHAPTER 4}

78. Kim B, Kim JS, Yoon Y, et al. Inhibition of Drp1-dependent mitochondrial division impairs myogenic differentiation. Am J Physiol Regul Integr Comp Physiol 2013;305(8):R927-38.

79. Barbieri E, Battistelli M, Casadei L, et al. Morphofunctional and Biochemical Approaches for Studying Mitochondrial Changes during Myoblasts Differentiation. J Aging Res 2011;2011:845379.

80. Koopman R, Ly CH, Ryall JG. A metabolic link to skeletal muscle wasting and regeneration. Frontiers in physiology 2014;5:32.

81. Ceco E, Weinberg SE, Chandel NS, et al. Metabolism and Skeletal Muscle Homeostasis in Lung Disease. Am J Respir Cell Mol Biol 2017;57(1):28-34.

82. Fortini $P$, Ferretti $C$, lorio $E$, et al. The fine tuning of metabolism, autophagy and differentiation during in vitro myogenesis. Cell Death Dis 2016;7:e2168.

83. Fortini $P$, lorio E, Dogliotti $E$, et al. Coordinated Metabolic Changes and Modulation of Autophagy during Myogenesis. Frontiers in physiology 2016;7:237.

84. Abreu P, Mendes SV, Ceccatto VM, et al. Satellite cell activation induced by aerobic muscle adaptation in response to endurance exercise in humans and rodents. Life Sci 2017;170:3340. 
SUPPLEMENTARY MATERIAL

Table S1. | Primary antibody list

\begin{tabular}{|c|c|c|c|c|}
\hline Target & Species & Supplier & Product No. & RRID \\
\hline Laminin & Mouse (histo) & Sigma & L9393 & AB_477163 \\
\hline PAX7 & Mouse (histo) & DSHB & AB528428 & AB_528428 \\
\hline AKT1 & Human & Cell signaling & 2920 & AB_1147620 \\
\hline AKT1 (Ser473) & Human & Cell signaling & 4060 & AB_2315049 \\
\hline BECN1 & Human & Novus Biologicals & NB1 10-87318SS & $A B \_1236726$ \\
\hline DNM1L & Human & Cell signaling & 8570 & AB_10950498 \\
\hline EIF4EBP1 & Human & Cell signaling & 9452 & AB_331692 \\
\hline EIF4IBP1 (Ser65) & Human & Cell Signaling & 9451 & AB_330947 \\
\hline F0X01 & Human & Cell signaling & 2880 & AB_2106495 \\
\hline FOXO3 & Human & Cell signaling & 2497 & $A B \_836876$ \\
\hline FOXO1 (Tyr24)/FOXO3 (Tyr32) & Human & Cell Signaling & 9464 & AB_329842 \\
\hline HK2 & Human & Cell Signaling & 2867 & AB_2232946 \\
\hline MSTN & Human & Santa Cruz & SC-6885-R & AB_640938 \\
\hline mTOR & Human & Cell signaling & 2983 & AB_2105622 \\
\hline mTOR (Ser2448) & Human & Cell Signaling & 2971 & AB_330970 \\
\hline NRF 1 & Human & Abcam & AB175932 & AB_2629496 \\
\hline OXPHOS complexes & Human & MitoSciences & MS604 & AB_2629281 \\
\hline PPARGC1A & Human & Cell Signaling & 2178 & AB_823600 \\
\hline RPS6K & Human & Cell singaling & 2317 & $A B \_2238583$ \\
\hline RPS6K (Ser235/236) & Human & Cell singaling & 4858 & AB_916156 \\
\hline TFAM & Human & Calbiochem & DR1071 & $A B \_10632341$ \\
\hline TOMм20 & Human & Abcam & AB186734 & AB_2716623 \\
\hline BNIP3 & Human/Mouse & Cell signaling & 3769 & AB_2259284 \\
\hline BNIP3L & Human/Mouse & Cell signaling & 12396 & $A B \_2688036$ \\
\hline FUNDC1 & Human/Mouse & Santa Cruz & SC-133597 & AB_10609242 \\
\hline GABARAPL1 & Human/Mouse & Proteintech & 11010-1-AP & AB_2294415 \\
\hline MAP1LC3B & Human/Mouse & Cell signaling & 2775 & AB_915950 \\
\hline PRKN & Human/Mouse & Cell Signaling & 4211 & AB_2159920 \\
\hline SQSTM1 & Human/Mouse & Cell Signaling & 5114 & $A B \_10624872$ \\
\hline ULK1 & Human/Mouse & Cell Signaling & 8054 & $A B \_11178668$ \\
\hline pULK (ser556)/pULK1 (ser555) & Human/Mouse & Cell Signaling & 5869 & AB_10707365 \\
\hline pULK (ser758)/pULK1 (ser757) & Human/Mouse & Cell Signaling & 6888 & $A B \_10829226$ \\
\hline
\end{tabular}




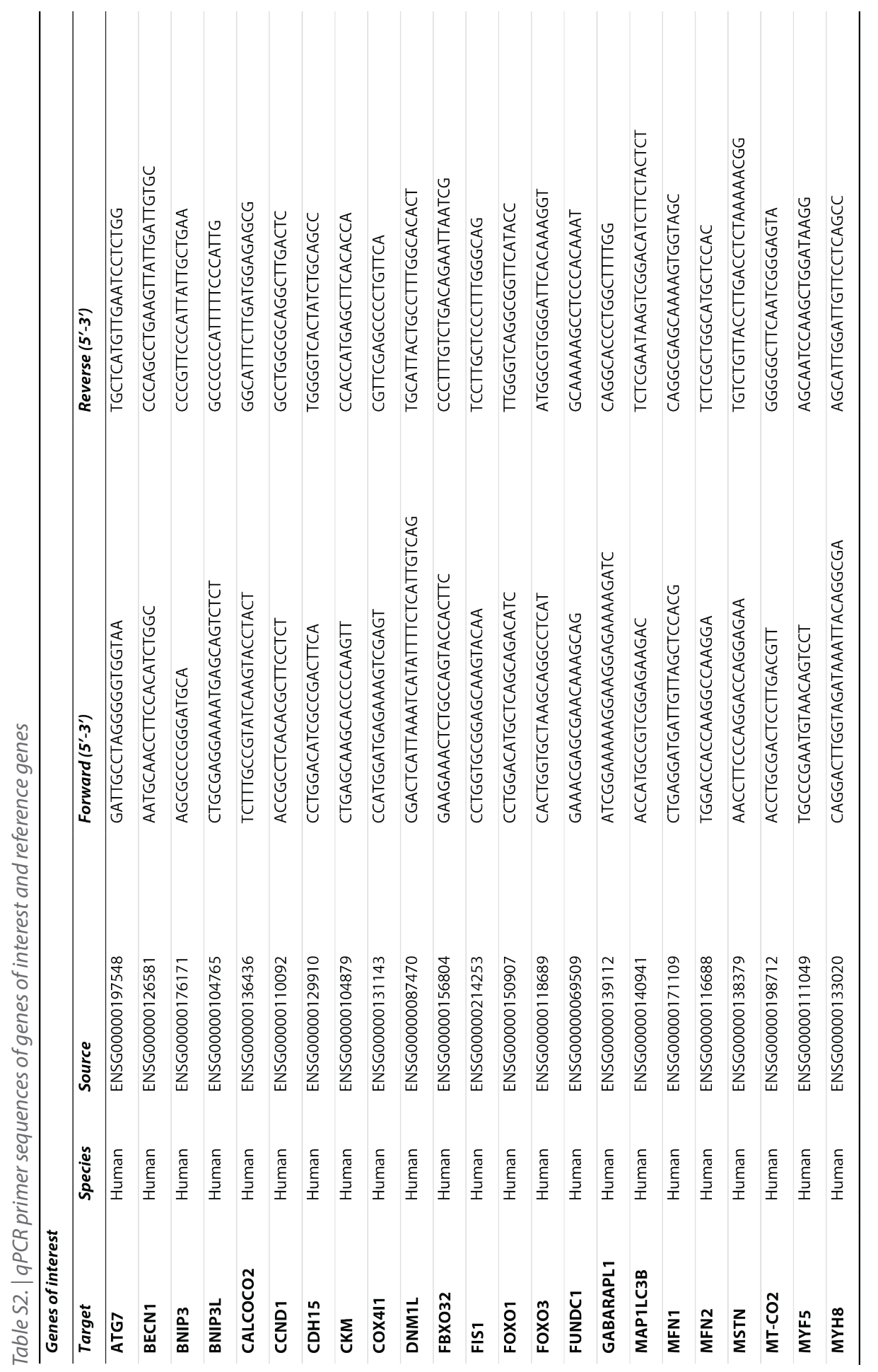




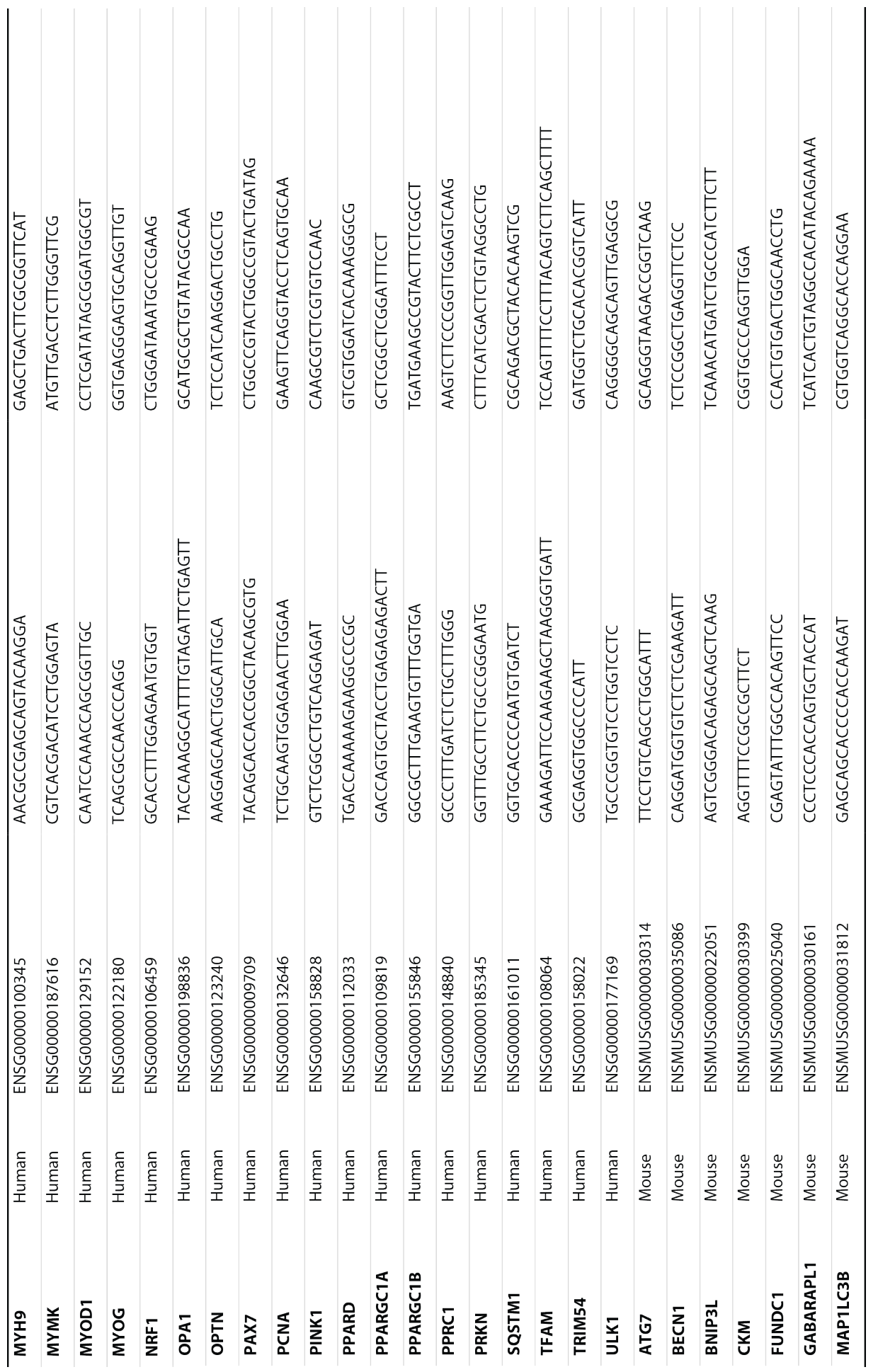




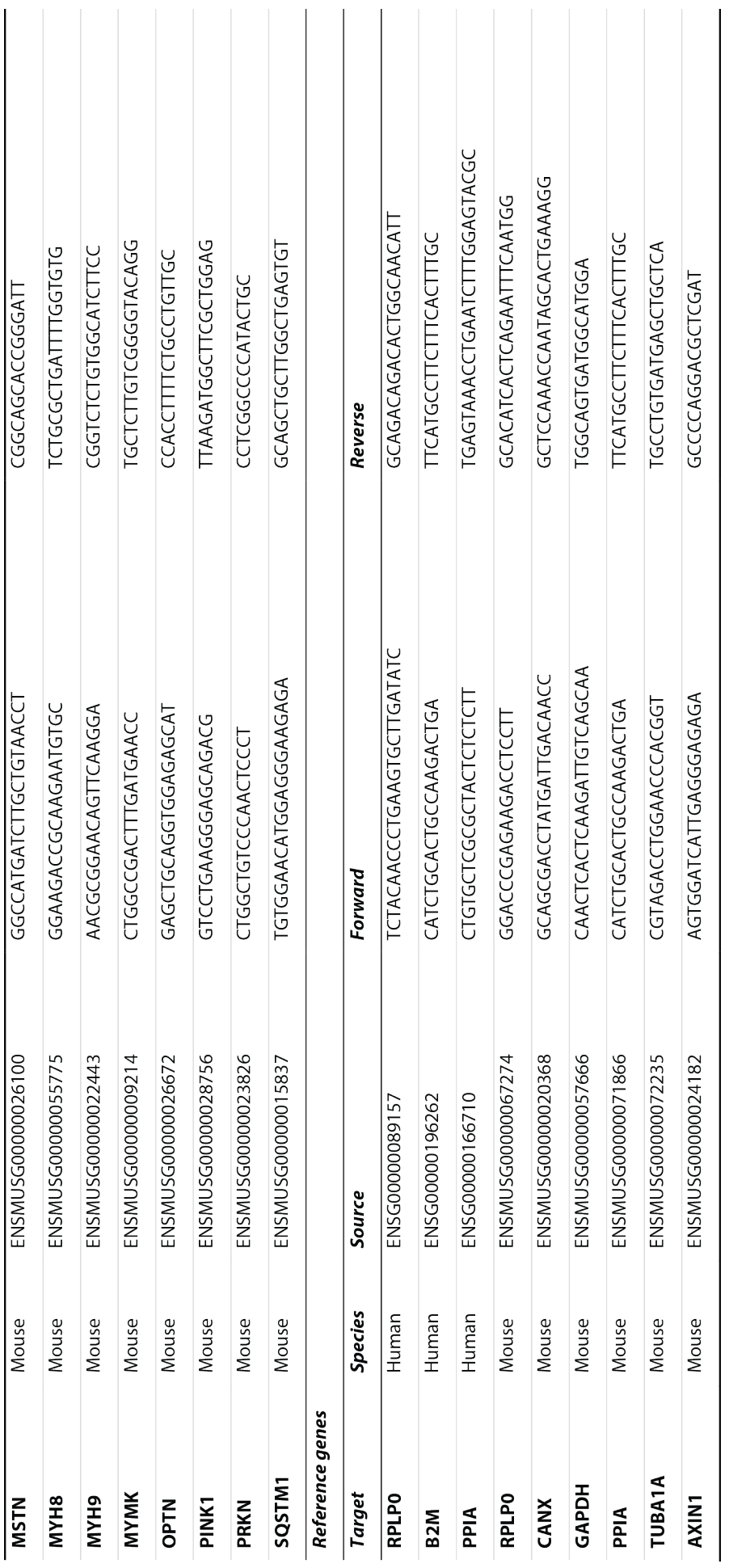


Table S3. | Cluster composition

\begin{tabular}{|c|c|c|c|c|c|c|}
\hline \multicolumn{2}{|c|}{ Process } & Cluster 1 & Cluster 2 & Cluster 3 & Cluster 4 & Total \\
\hline \multicolumn{7}{|c|}{ Protein turnover } \\
\hline \multirow{2}{*}{ Protein synthesis } & Count & 7 & 0 & 1 & 4 & 12 \\
\hline & $\%$ within process & $58.3 \% * *$ & $0.0 \%$ & $8.3 \%$ & $33.3 \%$ & $100.0 \%$ \\
\hline \multirow{2}{*}{$\begin{array}{l}\text { Ubiquitin } \\
\text { proteasome }\end{array}$} & Count & 4 & 0 & 1 & 2 & 7 \\
\hline & $\%$ within process & $57.1 \%$ & $0.0 \%$ & $14.3 \%$ & $28.6 \%$ & $100.0 \%$ \\
\hline \multirow{2}{*}{ Autophagy } & Count & 9 & 0 & 4 & 6 & 19 \\
\hline & $\%$ within process & $47.4 \%$ & $0.0 \%$ & $21.1 \%$ & $31.6 \%$ & $100.0 \%$ \\
\hline \multicolumn{7}{|c|}{ Mitochondrial turnover } \\
\hline Mitochondrial & Count & 1 & 3 & 6 & 2 & 12 \\
\hline biogenesis & $\%$ within process & $8.3 \%$ & $25.0 \%$ & $50.0 \%$ & $16.7 \%$ & $100.0 \%$ \\
\hline \multirow{2}{*}{ Mitophagy } & Count & 1 & 2 & 4 & 2 & 9 \\
\hline & $\%$ within process & $11.1 \%$ & $22.2 \%$ & $44.4 \%$ & $22.2 \%$ & $100.0 \%$ \\
\hline \multirow{2}{*}{$\begin{array}{l}\text { Mitochondrial } \\
\text { mass }\end{array}$} & Count & 0 & 0 & 0 & 6 & 6 \\
\hline & $\%$ within process & $0.0 \%$ & $0.0 \%$ & $0.0 \%$ & $100.0 \% * *$ & $100.0 \%$ \\
\hline \multirow{2}{*}{$\begin{array}{l}\text { Mitochondrial } \\
\text { dynamics }\end{array}$} & Count & 0 & 1 & 3 & 2 & 6 \\
\hline & $\%$ within process & $0.0 \%$ & $16.7 \%$ & $50.0 \%$ & $33.3 \%$ & $100.0 \%$ \\
\hline \multicolumn{7}{|c|}{ Myonuclear turnover } \\
\hline \multirow{2}{*}{ Myogenesis } & Count & 2 & 3 & 4 & 4 & 13 \\
\hline & $\%$ within process & $15.4 \%$ & $23.1 \%$ & $30.8 \%$ & $30.8 \%$ & $100.0 \%$ \\
\hline \multirow{2}{*}{$\begin{array}{l}\text { Muscle mass and } \\
\text { function }\end{array}$} & Count & 1 & 4 & 2 & 2 & 9 \\
\hline & $\%$ within process & $11.1 \%$ & $44.4 \%$ & $22.2 \%$ & $22.2 \%$ & $100.0 \%$ \\
\hline \multirow{2}{*}{ Total } & Count & 25 & 13 & 25 & 30 & 93 \\
\hline & $\%$ within process & $26.9 \%$ & $14.0 \%$ & $26.9 \%$ & $32.3 \%$ & $100.0 \%$ \\
\hline
\end{tabular}

Distribution of markers and mediators associated with the indicated processes, presented as the number (counts) or percentage (\% within process) of markers per cluster. ${ }^{* *} p<0.01$ Pearson's chi-square post-hoc based on adjusted residuals, with Bonferroni correction. 


\section{CHAPTER 4}

A

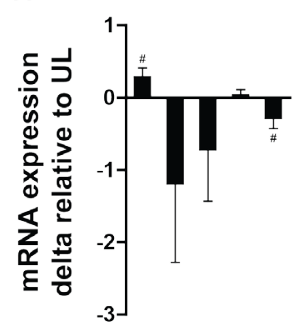

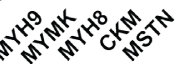

\section{4}

E

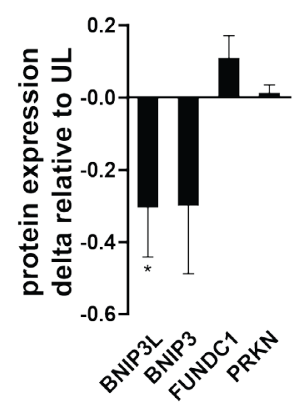

B

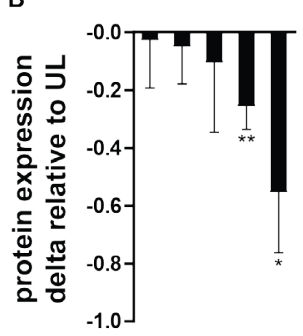

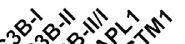

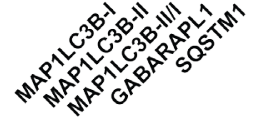

$\mathbf{F}$

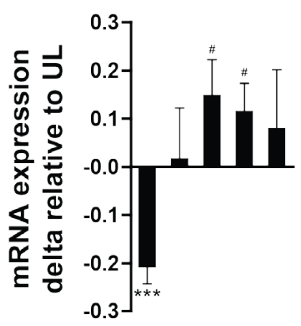

C
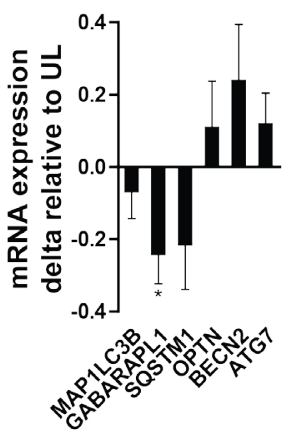

D
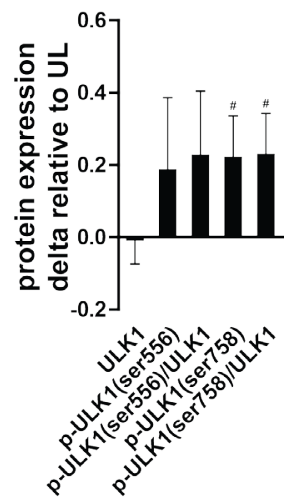

Figure S1. |Change in MRNA and protein expression in human M. vastus lateralis upon 1-week recovery from disuse. Relative change in $m R N A$ and protein expression upon reloading $(R L)$, in fold change compared to unloading (UL). A. Relative mRNA expression of markers and mediators of late myogenesis. B. Relative protein expression of indicators of autophagosome formation. C. Relative $m R N A$ expression of autophagy-related genes. D. Relative protein expression ofmarkers of autophagy initiation. E. Relative protein expression of mitophagy regulators and mediators. F. Relative mRNA expression of mitophagy-related genes. $\# p<0.1,{ }^{*} p<0.05,{ }^{* *} p<0.01,{ }^{* * *} p<0.001$ compared to UL. 
DISTINCT SKELETAL MUSCLE MOLECULAR RESPONSES TO PULMONARY REHABILITA

J Cachexia Sarcopenia Muscle 2018. Accepted

ANITA E.M. KNEPPERS ROY A.M. HAAST

RAMON C.J. LANGEN

LEX B. VERDIJK

PIETER A. LEERMAKERS

HARRY R. GOSKER

LUC J.C. VAN LOON

MITJA LAINSCAK

\& ANNEMIE M.W.J. SCHOLS 


\section{CHAPTER 5}

\section{ABSTRACT}

Background: Pulmonary rehabilitation (PR) is a cornerstone in the management of COPD, targeting skeletal muscle to improve functional performance. However, there is substantial inter-individual variability in the effect of PR on functional performance, which cannot be fully accounted for by generic phenotypic factors. We performed an unbiased integrative analysis of the skeletal muscle molecular responses to PR in COPD patients, and comprehensively characterized their baseline pulmonary and physical function, body composition, blood profile, comorbidities, and medication use.

Methods: M. vastus lateralis biopsies were obtained from 51 COPD patients (Age $64 \pm 1$ years, Sex 73\% males, FEV, 34 (26-41) \%pred.) before and after 4 weeks high-intensity supervised in-patient PR. Muscle molecular markers were grouped by networkconstrained clustering, and their relative changes in expression values - assessed by qPCR and Western blot - were reduced to process scores by principal component analysis. Patients were subsequently clustered based on these process scores. Pre- and post-PR functional performance was assessed by incremental cycle ergometry and 6-min walking test (6MWT).

Results: Eight molecular processes were discerned by network-constrained hierarchical clustering of the skeletal muscle molecular rehabilitation responses. Based on the resulting process scores, four clusters of patients were identified by hierarchical cluster analysis. Two major patient clusters differed in PR-induced Autophagy $(p<0.001)$, Myogenesis ( $p=0.014)$, Glucocorticoid signaling $(p<0.001)$, and Oxidative metabolism regulation $(\mathrm{p}<0.001)$, with Cluster $1(\mathrm{C} 1 ; n=29)$ overall displaying a more pronounced change in marker expression than Cluster $2(C 2 ; n=16)$. General baseline characteristics did not differ between clusters. Following PR, both 6-min walking distance $(+26.5 \pm 8.3$ $m, p=0.003)$ and peak load on the cycle ergometer test $(+9.7 \pm 1.9 \mathrm{~W}, \mathrm{p}<0.001)$ were improved. However, the functional improvement was more pronounced in $\mathrm{C} 1$, as a higher percentage of patients exceeded the minimal clinically important difference in peak workload (61 versus $21 \%, \mathrm{p}=0.022$ ), and both peak workload and 6MWT (52 versus $8 \%, p=0.008$ ) upon PR.

Conclusions: We identified patient groups with distinct skeletal muscle molecular responses to rehabilitation, associated with differences in functional improvements upon PR.

\section{Acknowledgements}

The authors wish to thank Marco Kelders and Chiel de Theije for their excellent technical assistance in performing the molecular analyses. 


\section{BACKGROUND}

Chronic obstructive pulmonary disease (COPD) is increasingly recognized as a complex chronic disease with extra-pulmonary manifestations and comorbidities, including skeletal muscle dysfunction [1]. Skeletal muscle dysfunction in COPD arises from structural and metabolic alterations such as a loss of muscle mass, a shift in fiber type distribution, decreased oxidative capacity, and mitochondrial dysfunction [2]. Importantly, besides affecting physical functioning and health-related quality of life [3], skeletal muscle dysfunction is a predictor of mortality independent of lung function $[4,5]$.

In clinically stable COPD patients, exercise training is currently the most potent intervention to improve skeletal muscle function. As such, exercise training forms a cornerstone in pulmonary rehabilitation (PR) in patients with COPD. However, despite the overall positive effects of exercise training on muscle mass and function at the group level, there is substantial inter-individual variability in the effect of PR on skeletal muscle function $[2,6-10]$. The sources of this variability currently remain unexplained, but may be better understood when we improve our comprehension of the underlying skeletal muscle molecular response pattern. In the older population, which is prone to the loss of muscle mass and strength (i.e., sarcopenia), the exercise response can be blunted due to impaired skeletal muscle regeneration [11]. This potentially results from an impaired induction of skeletal muscle remodeling-related processes, as reflected by a blunted induction of protein degradation [12], anabolic resistance [13], and a delayed or blunted satellite cell response [11]. Similarly, the exercise response in COPD patients may be limited by impaired satellite-cell mediated skeletal muscle regeneration [14]. Furthermore, we recently showed an elevated basal activity of skeletal muscle remodeling-related processes in COPD patients [15]. This may hinder the normal induction of these processes during skeletal muscle recovery after disuse or upon exercise training [12].

Several studies assessed molecular markers of remodeling-related processes upon exercise training in COPD patients [10, 16-18], but none report a clear differential rehabilitation response between predefined phenotypic categories, e.g. based on body composition. This may be partially due to the limited sample size in most of these studies, or the individual assessment of a limited selection of markers, which often display a large variation. Nevertheless, age, body composition, and comorbidities such as metabolic and heart diseases, have been weakly associated with the functional response to rehabilitation $[16,19,20]$. Other studies however, report inconclusive or 


\section{CHAPTER 5}

even contradicting results $[6-9,17,21,22]$, strongly indicating that these phenotypic factors do not fully account for the variability in rehabilitation responses.

In the current study, we take a clustering approach to - for the first time — unbiasedly address inter-individual heterogeneity in the skeletal muscle molecular response to PR of patients with advanced COPD.

\section{METHODS}

\section{Study design and participants}

We selected COPD patients from a prospective observational study conducted in the inpatient PR unit at the University Clinic Golnik. The study design has previously been published [23] (ClinicalTrials.gov identifier: NCT02550808). The study was approved by the Slovenian National Medical Ethics Committee (Ljubljana, Slovenia), was carried out in accordance with the ethical standards laid down in the 1964 Declaration of Helsinki and its later amendments, and all participants provided written informed consent. Patient selection for the current analysis was based on completion of the 4-week rehabilitation program, and availability of both a pre- and post-rehabilitation muscle biopsy specimens, yielding 51 patients.

\section{Pulmonary and physical function}

Spirometry was used to obtain $\mathrm{FEV}_{1}, \mathrm{FVC}$, and their ratio (FEV /FVC) [24]. Physical function was assessed with the 6-min walking test (6MWT) [25], and by measurement of peak load $(\mathrm{W})$ and peak oxygen consumption $\left(\mathrm{VO}_{2}, \mathrm{~mL} / \mathrm{kg} / \mathrm{min}\right)$ by an incremental load cycling cardiopulmonary exercise test (CPET) [26]. In addition, the improvements in physical function were categorized by the minimal clinically important difference (MCID; 6MWT: 25m [27], peak load: 10W [28]).

\section{Body composition}

Whole body dual-energy x-ray absorptiometry was used to assess total and appendicular (i.e., arms and legs) fat mass and fat-free mass at baseline, as previously described [23]. Fat Mass Index (FMI), Fat-Free Mass Index (FFMI), and Appendicular Skeletal muscle Mass Index (ASMI) were calculated as the ratio of mass to height ${ }^{2}(\mathrm{~kg} /$ $\left.\mathrm{m}^{2}\right)$. Presence of muscle mass depletion was defined by the cut-offs for ASMI $(<7.23$ $\mathrm{kg} / \mathrm{m}^{2}$ for men; $<5.76 \mathrm{~kg} / \mathrm{m}^{2}$ for women) [29]. Bioelectrical impedance (BIA; QuadScan 4000; Bodystat, Douglas, United Kingdom) was performed pre- and post-PR as previously described [23], and was used to assess the change in FMI and FFMI upon PR. 


\section{Skeletal muscle molecular analyses}

Pre- and post-PR biopsies were obtained from the vastus lateralis muscle of the dominant leg by needle biopsy, one day ( $\geq 20 \mathrm{~h}$ ) after the last exercise test. Muscle tissue was snap-frozen in liquid nitrogen, and stored at $-80^{\circ} \mathrm{C}$ until processed for molecular analyses. mRNA levels and protein abundance of mediators and regulators of muscle mass and metabolism were determined by RT-qPCR and Western blotting, respectively. Details are provided in the supplementary methods.

\section{Data analysis}

\section{Clustering}

Details on the clustering analyses are provided in the supplementary methods. Briefly, molecular rehabilitation responses, calculated as the percentage change from prerehabilitation values, were grouped by network-constrained hierarchical clustering of the markers. The resulting processes were named based on enrichment of Gene Ontology (GO) biological processes. Data dimensionality was reduced by computing a single score for each process using principle component analysis (PCA). Subsequently, patients were hierarchically clustered based on their individual process scores. Analyses were performed in Python using the scikit-learn library, and results were visualized using Graph-tool.

\section{Statistics}

After checking for normality, rehabilitation responses were tested by paired-sample t-test. Between-group differences were tested by independent-sample t-test (2 groups), by one-way ANOVA with Bonferroni post-hoc comparisons ( $>2$ groups), or by their non-parametric or categorical equivalents. Correlations between molecular processes were tested using Pearson's correlation coefficient, and between-group differences in correlations were tested by a Fisher r-to-z transformation. Analyses were performed using SPSS Statistics (version 22.0, IBM Corp., Armonk, NY, USA). A p-value $<0.05$ was considered statistically significant.

\section{RESULTS}

\section{General characteristics}

51 patients were included in the analyses, with subject characteristics displayed in Table 1. The patient group consisted mostly out of males, current smokers, and $76 \%$ of patients completed the PR without an exacerbation. Based on the GOLD classification [30], most patients had severe (stage III; 53\%) to very severe COPD (stage IV; $33 \%$ ), and $60 \%$ of patients had muscle mass depletion. 


\section{CHAPTER 5}

Table 1. | Baseline subject characteristics.

\begin{tabular}{|c|c|c|c|c|}
\hline & Whole group & Cluster 1 & Cluster 2 & p-value* \\
\hline & $\mathrm{n}=51$ & $\mathrm{n}=29$ & $\mathrm{n}=16$ & \\
\hline \multicolumn{5}{|l|}{ Demographics } \\
\hline Age, years & $64 \pm 1$ & $64 \pm 2$ & $65 \pm 2$ & ns \\
\hline Sex, $\mathrm{m} / \mathrm{f}(\%$ male) & $37 / 14(73)$ & $21 / 8(72)$ & $11 / 5(69)$ & ns \\
\hline Smoking status & & & & $<0.1$ \\
\hline Current, n (\%) & $44(88)$ & $24(83)$ & $16(100)$ & \\
\hline Former, n (\%) & $6(12)$ & $5(17)$ & $0(0)$ & \\
\hline Completion of PR & & & & ns \\
\hline Without exacerbation, n (\%) & $39(76)$ & $23(79)$ & $11(69)$ & \\
\hline With exacerbation, $\mathrm{n}(\%)$ & $12(24)$ & $6(21)$ & $5(31)$ & \\
\hline \multicolumn{5}{|l|}{ Pulmonary function } \\
\hline $\mathrm{FEV}_{1}, \%$ predicted $\dagger$ & $34[26-41]$ & $34[26-41]$ & $38[29-44]$ & ns \\
\hline FVC, \% predicted & $79 \pm 3$ & $80 \pm 3$ & $77 \pm 5$ & ns \\
\hline $\mathrm{FEV}_{1} / \mathrm{FVC}, \% \dagger$ & $33[26-42]$ & $31[26-40]$ & $33[28-46]$ & ns \\
\hline \multicolumn{5}{|l|}{ Physical function } \\
\hline $6 \mathrm{MWT}, \mathrm{m}$ & $344.68 \pm 15.44$ & $352.59 \pm 22.11$ & $348.00 \pm 20.94$ & ns \\
\hline Peak load, W & $62.96 \pm 3.30$ & $62.81 \pm 4.81$ & $65.20 \pm 5.51$ & ns \\
\hline Peak $\mathrm{VO}_{2}, \mathrm{~mL} / \mathrm{kg} / \mathrm{min}$ & $11.75 \pm 0.58$ & $12.31 \pm 0.85$ & $11.35 \pm 0.77$ & ns \\
\hline \multicolumn{5}{|l|}{ Body composition } \\
\hline $\mathrm{BMI}, \mathrm{kg} / \mathrm{m}^{2}$ & $24.8 \pm 0.6$ & $24.2 \pm 0.7$ & $25.5 \pm 1.3$ & ns \\
\hline $\mathrm{FMI}, \mathrm{kg} / \mathrm{m}^{2}$ & $7.8 \pm 0.3$ & $7.4 \pm 0.4$ & $8.3 \pm 0.7$ & ns \\
\hline $\mathrm{FFMI}, \mathrm{kg} / \mathrm{m}^{2}$ & $16.9 \pm 0.4$ & $16.7 \pm 0.5$ & $16.9 \pm 0.9$ & ns \\
\hline ASMI, $\mathrm{kg} / \mathrm{m}^{2}$ & $6.6 \pm 0.2$ & $6.5 \pm 0.2$ & $6.9 \pm 0.4$ & ns \\
\hline Sarcopenia, n (\%) & $30(60)$ & $20(69)$ & $7(47)$ & ns \\
\hline \multicolumn{5}{|l|}{ Blood profile } \\
\hline Triglycerides, $\mathrm{mmol} / \mathrm{L}$ & $1.28 \pm 0.08$ & $1.16 \pm 0.10$ & $1.46 \pm 0.14$ & $<0.1$ \\
\hline $\mathrm{HDL}$ cholesterol, $\mathrm{mmol} / \mathrm{L}$ & $1.77 \pm 0.09$ & $1.80 \pm 0.10$ & $1.73 \pm 0.16$ & ns \\
\hline LDL cholesterol, mmol/L & $3.01 \pm 0.15$ & $2.77 \pm 0.19$ & $3.25 \pm 0.26$ & ns \\
\hline LDL/HDL, ratio & $1.94 \pm 0.14$ & $1.65 \pm 0.14$ & $2.21 \pm 0.27$ & $<0.1$ \\
\hline HOMA-IR† & $3.40[1.80-6.70]$ & $3.70[1.95-5.75]$ & $2.90[1.85-8.00]$ & ns \\
\hline \multicolumn{5}{|l|}{ Comorbidities } \\
\hline Long term oxygen therapy, $\mathrm{n}(\%)$ & $20(39)$ & $11(38)$ & $5(31)$ & ns \\
\hline Heart failure, $\mathrm{n}(\%)$ & $8(16)$ & $5(17)$ & $2(13)$ & ns \\
\hline Ischemic heart disease, n (\%) & $4(8)$ & $3(10)$ & $0(0)$ & ns \\
\hline Atrial fibrillation, $\mathrm{n}(\%)$ & $1(2)$ & $1(3)$ & $0(0)$ & ns \\
\hline Arterial hypertension, $\mathrm{n}(\%)$ & $21(41)$ & $10(34)$ & $8(50)$ & ns \\
\hline Cholesterolemia, n (\%) & $14(27)$ & $6(21)$ & $5(31)$ & ns \\
\hline Type 2 diabetes mellitus, $\mathrm{n}(\%)$ & $7(14)$ & $5(17)$ & $1(6)$ & ns \\
\hline
\end{tabular}

6MWT, six-minute walk test; ASMI, appendicular skeletal muscle mass index; BMI, body mass index; FEV ${ }_{1}$, forced expiratory volume in 1s; FFMI, fat-free mass index; FMI, fat mass index; FVC, forced vital capacity; HDL, high-density lipoprotein; HOMA-IR, homeostatic model of insulin resistance; $L D L$, low-density lipoprotein; $P R$, pulmonary rehabilitation. Due to the insufficient sample size, individual values for Cluster $3(n=4)$ and Cluster $4(n=2)$ are not depicted in this table. Data expressed as mean \pm SEM unless indicated otherwise. ${ }^{*}$ Significance of between group (Cluster 1 versus Cluster 2) comparisons assessed by independent sample t-test, Mann-Whitney $U$ test, or Chi-square test. tMedian [IQR]. 


\section{Functional and skeletal muscle molecular rehabilitation responses in COPD patients}

Following PR, both 6-min walking distance $(+26.5 \pm 8.3 \mathrm{~m}, \mathrm{p}<0.01)$ and peak load on the cycle ergometer test $(+9.7 \pm 1.9 \mathrm{~W}, \mathrm{p}<0.001)$ were improved. Moreover, 25 patients (51\%) exceeded the MCID on the 6MWT, and 17 patients (40\%) exceeded the MCID for peak load upon PR.

Using data from all 51 patients, eight molecular processes (i.e., P1-P8) were discerned by network-constrained hierarchical clustering of the skeletal muscle molecular rehabilitation responses (Figure 1a, Table S1), and were named based on GO-term overrepresentation as indicated in Table S1. In general, P1 (Autophagy) related mRNA expression was decreased, whereas Autophagy-related protein phosphorylation and expression were increased after PR (Figure 1b). Furthermore, P2 (AKT/mTOR signaling) related mRNA expression, and protein phosphorylation and expression were predominantly increased upon rehabilitation (Figure 1b). Similarly, P3 (Myogenesis) related mRNA and protein expression, and P4 (Oxidative phosphorylation capacity (OXCAP)) and P5 (Mitophagy, FUNDC1/DNM1L) related protein expression were increased upon rehabilitation, whereas P6 (Mitophagy, PRKN) related protein expression was decreased (Figure 1b). Furthermore, P7 (Glucocorticoid signaling) related inhibitory phosphorylation seemed to increase upon rehabilitation, and in line with this, Glucocorticoid signaling-related downstream mRNA expression was decreased (Figure 1b). Despite the significant decrease in mRNA expression of transcriptional regulators of mitochondrial biogenesis (TFAM and PPRC1), and the increase in a regulator of mitochondrial quality (PINK1), P8 (Oxidative metabolism regulation) related markers displayed no consistent overall pattern of change upon rehabilitation (Figure $1 \mathrm{~b}$ ). 


\section{CHAPTER 5}
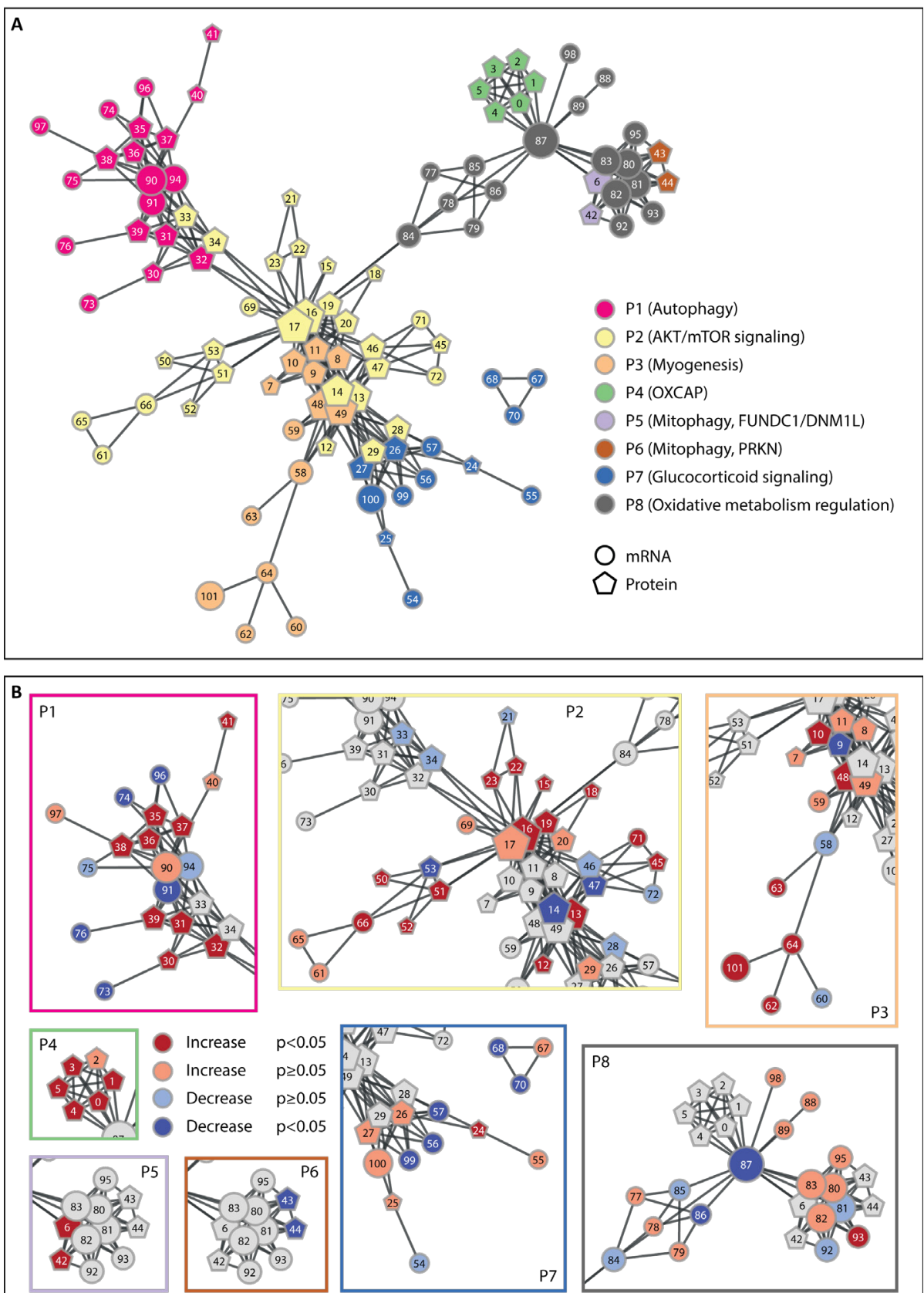

Figure 1.|Molecular network, and network-constrained hierarchical clustering of molecular rehabilitation responses. a) Network-constrained clusters revealing 8 distinct processes $(P)$, as indicated with different colors. b) Molecular rehabilitation responses. The literature-based molecular network is indicated as lines between molecular markers. Circles represent mRNA markers; pentagons represent protein markers. Numbers correspond to individual markers as depicted in Table S1. 


\section{Identification of patient clusters with differential skeletal muscle molecular rehabilitation responses}

Both the functional and skeletal muscle molecular rehabilitation responses displayed a substantial inter-individual variation. To gain insight in the determinants of this variation, an unbiased approach was used to classify patients based on their molecular rehabilitation responses. To this end, one process score per identified molecular process was computed by PCA, with component loadings indicated in Table S2. Four clusters (C1-4) of patients were identified by hierarchical cluster analysis based on process scores, using a cutoff based on the local optimum in silhouette score (Figure $2 a-b)$. C3 and C4 had an insufficient sample size ( $n=4$ and $n=2$, respectively) for the statistical detection of relevant group differences, and were therefore omitted in further analyses.

$\mathrm{C} 1(\mathrm{n}=29)$ and C2 $(\mathrm{n}=16)$ differed in rehabilitation-induced modulation of Autophagy, Myogenesis, Glucocorticoid signaling, and Oxidative metabolism regulation, and tended to differ by regulation of OXCAP (Figure 2c, Table S3). Specifically, the difference in rehabilitation-induced change in Autophagy markers between $\mathrm{C} 1$ and $\mathrm{C} 2$ was mainly reflected by a differential change in SQSTM1, MAP1LC3B, BECN1, BNIP3, ULK1, GABARAPL1, and OPTN mRNA expression (Table S4). Although, both clusters displayed an overall increase in Autophagy-related protein expression, a differential change in MAP1LC3BI, and a tendency towards a differential change in MAP1LC3BII/I were observed (Table S4). Conversely, the differential regulation of Myogenesis was only reflected by a significant difference in the rehabilitation-induced change in MYOD1 and $\mathrm{CDH} 15$ mRNA expression (Table S4). In contrast to Myogenesis, the differential regulation of Glucocorticoid signaling consisted of a differential rehabilitation-induced change in all related markers, although $\mathrm{FOXO} 3 \mathrm{mRNA}$ did not reach significance (Table S4). The difference in the rehabilitation-induced modulation of Oxidative metabolism regulation was reflected by a differential change in PINK1, OPA1, FIS1, COX4I1, MYH2, MFN1, TFAM, PPARGC1A, PPRC1, PPARGC1B, PRKN, MYH1, and SOD2 mRNA expression (Table S4). Interestingly, although the tendency towards a differential regulation in OXCAP was not apparent at the marker level, a significant rehabilitation-induced increase in NDUFB8, SDHB, MT-CO1, ATP5F1A, and TOMM20 protein expression was observed for C1, while these markers remained unaltered in C2 (Table S4). 


\section{CHAPTER 5}

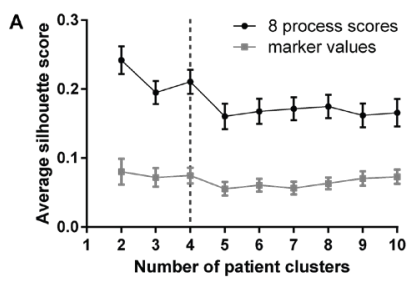

C
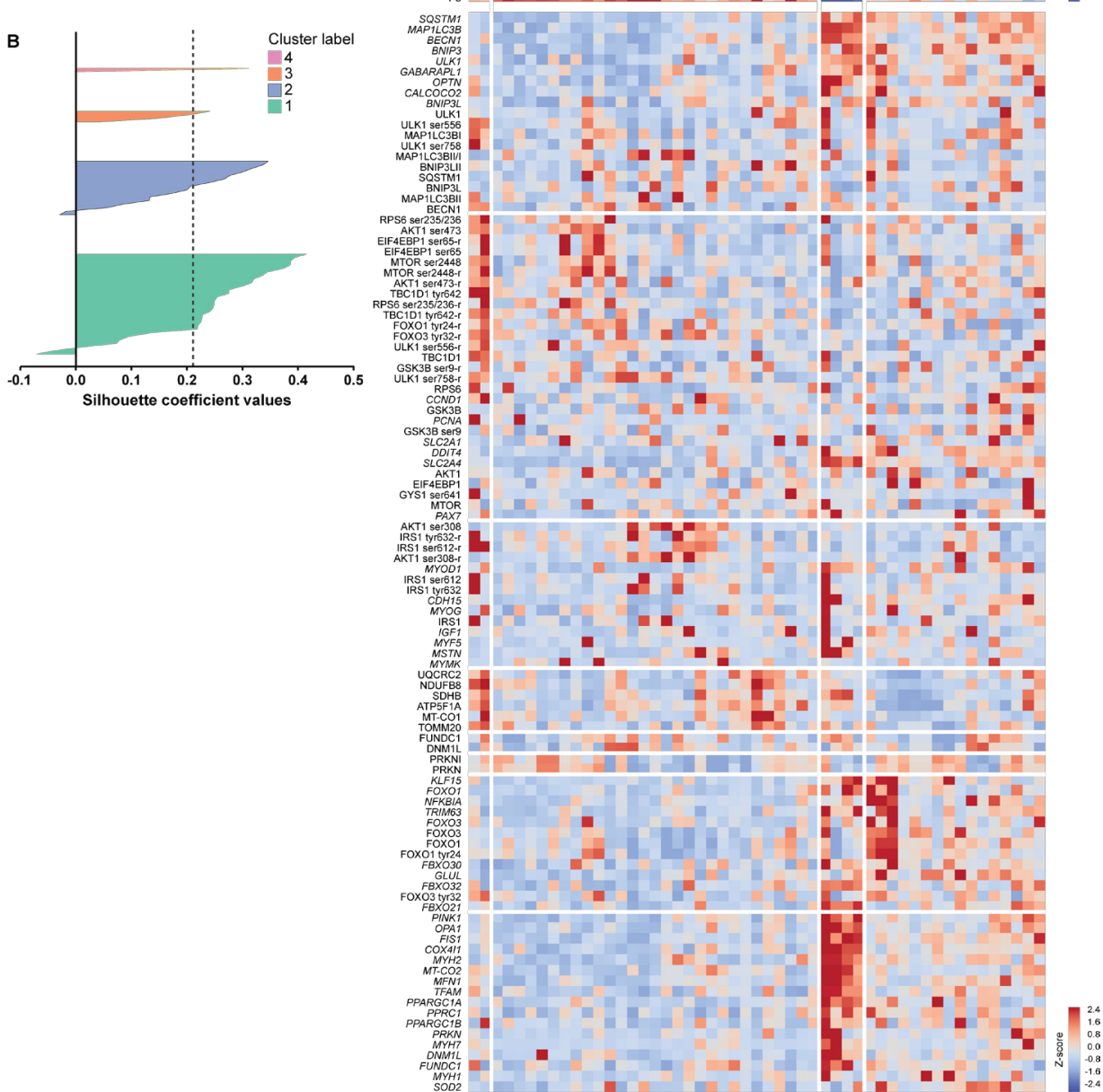

Figure 2. Hierarchical clustering of patients. a) Silhouette scores for $n$ clusters (mean \pm SEM). Clustering based on raw molecular rehabilitation responses (marker values; i.e., no data reduction) is indicated in grey; clustering based on 8 process scores is indicated in black. b) Patients individual silhouette coefficient values per cluster. c) Dendrogram and clustered heatmap of individual marker rehabilitation responses (Z-scores) and process scores. 


\section{Association between muscle molecular rehabilitation responses}

To assess whether a differential response at the process and marker level resulted in a differential coordination between processes, the correlations between scores of the eight identified processes were computed and compared between the two clusters (Figure 3).

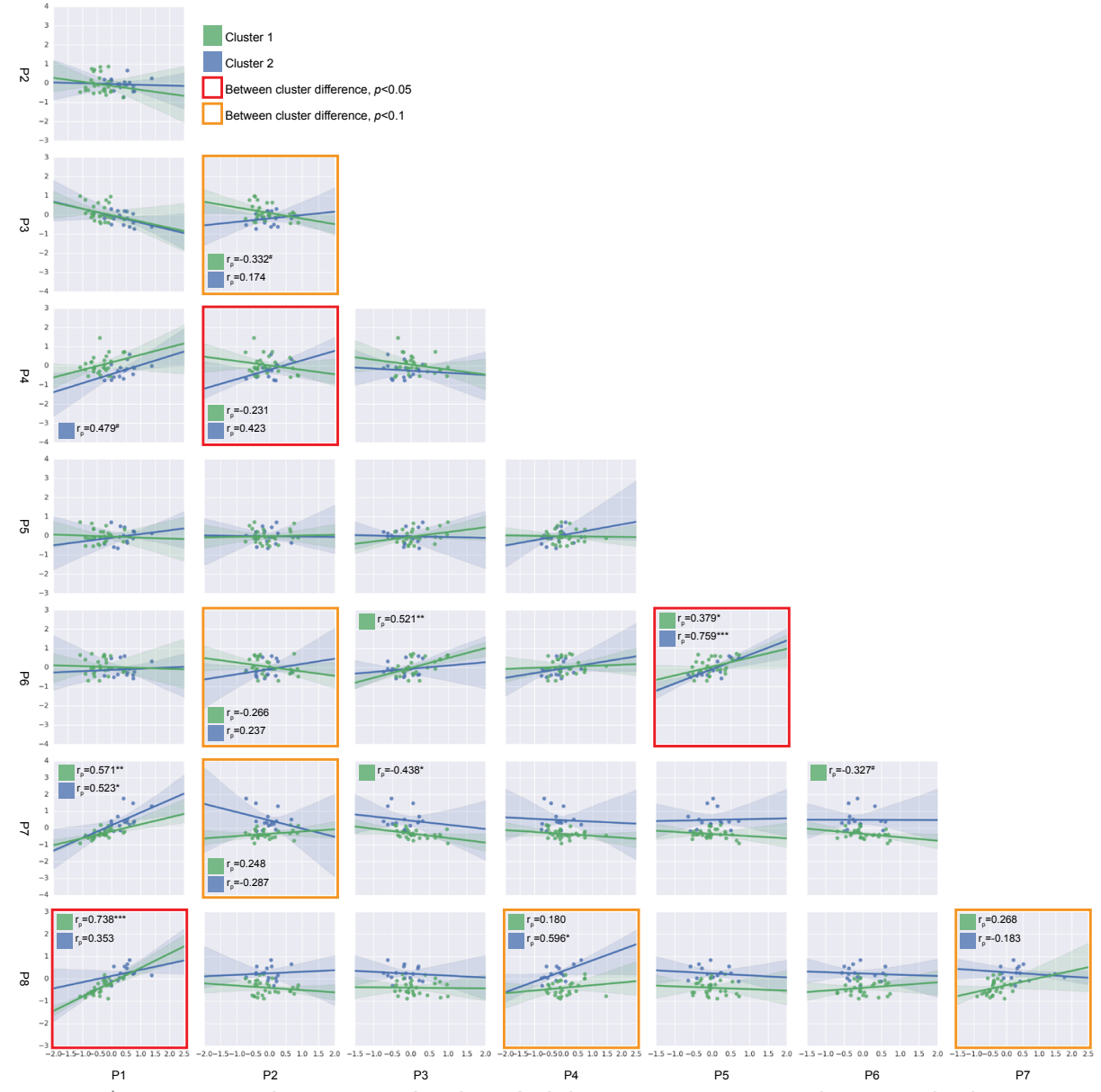

Figure 3. | Associations between molecular rehabilitation responses. Each square displays a scatter plot and regression line of the processes indicated on the $x$ and $y$ axis, for both Cluster 1 and Cluster 2. Differences between correlations were tested by a Fisher $r$-to-z transformation, and indicated with a red outline $(p<0.05)$, or orange outline $(p<0.1)$. Pearson correlation coefficients per cluster are depicted for differential correlations, and when $p<0.1$. $\# p<0.1,{ }^{*} p<0.05,{ }^{* *} p<0.01,{ }^{* * *} p<0.001$.

Differential correlations for C1 and C2 were observed between Autophagy and Oxidative metabolism regulation, between AKT/mTOR signaling and OXCAP, and between Mitophagy (FUNDC1/DNM1L) and Mitophagy (PRKN) (all $p<0.05$ ) (Figure 3). In 


\section{CHAPTER 5}

addition, AKT/mTOR signaling tended to be differentially correlated with Myogenesis $(p=0.06)$, Mitophagy (PRKN) $(p=0.07)$, and Glucocorticoid signaling $(p=0.05)$ (Figure 3$)$. Moreover, Oxidative metabolism regulation tended to be differentially correlated with $\operatorname{OXCAP}(p=0.07)$, and Glucocorticoid signaling $(p=0.09)$ (Figure 3).

\section{Cluster characteristics and functional rehabilitation responses}

C1 and C2 did not differ significantly in demographics, pulmonary function or body composition (Table 1), nor comorbidities or use of medication (Table 1, Table S5). Likewise, C1 and C2 did not differ in baseline values of the functional parameters (Table 1) and average rehabilitation-induced change in FFMI or FMI assessed by BIA (Figure S1). Furthermore, these clusters did not differ significantly in the average rehabilitation-induced change in 6MWT or peak load (Figure 4a-b). However, a pronounced and significant increase in 6MWT was observed in C1, whereas the change in 6MWT did not reach significance in C2 (Figure 4a). Similarly, a pronounced and significant rehabilitation-induced increase in peak load was observed in $\mathrm{C} 1$, but not in C2 (Figure 4b).

Categorization of rehabilitation responses based on the MCID revealed that the distribution of change in peak load differed between $\mathrm{C} 1$ and $\mathrm{C} 2$, with a relatively larger amount of patients exceeding the MCID in C1 (61 versus 21\%, $p<0.05$ ) (Figure 4c). In accordance, $\mathrm{C} 1$ contained relatively more patients that exceeded the MCID for both the 6 MWT and peak load than C2 (52 versus $8 \%, p<0.01$ ) (Figure $4 c$ ).

A

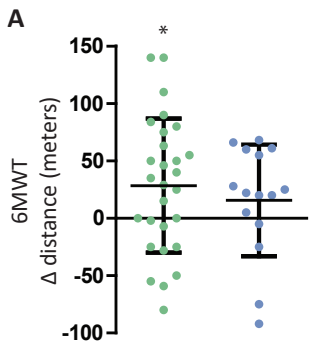

B

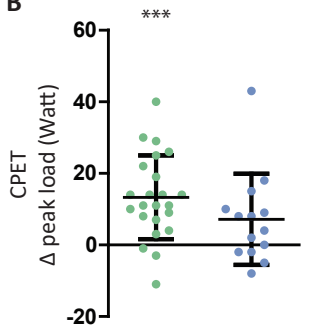

C

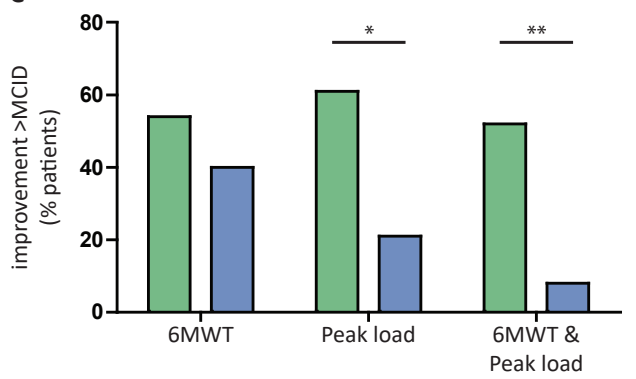

Figure 4. | Functional rehabilitation responses per cluster. Individual rehabilitation-induced changes. a) Distance (meters) walked in sixminute walk test (6MWT), $n=28 / 15$. b) Peak load (W) on a cycle ergometer test, $n=23 / 14$. Data expressed as mean $\pm S D$. c) Percentage of patients with a change in 6MWT, Peak load, or both, exceeding the minimal clinically important difference (MCID; 6MWT: 25m, peak load: 10W). ${ }^{*} p<0.05, \quad{ }^{* *} p<0.01, \quad{ }^{* * *} p<0.001$, indicating significance of withingroup rehabilitation responses, or significance of differences between indicated groups. 


\section{DISCUSSION}

To our knowledge, this study is the first to perform unbiased clustering of COPD patients based on their skeletal muscle molecular response to PR. Using this approach, we show that clustering based on the molecular response to PR leads to the identification of patient groups who differ by their functional response to PR.

\section{Variability in the response to pulmonary rehabilitation}

Study participants followed a supervised, high-intensity in-patient PR program, which targeted skeletal muscle using a comprehensive exercise training program [31]. In line with previous studies [32], PR improved physical performance assessed by 6MWT and peak workload on a cycle ergometer test, but the effect was highly variable. We measured a panel of molecular markers that are important for exercise-induced skeletal muscle mass and metabolic plasticity [33-36], and similarly, observed high variability in the rehabilitation-induced changes in skeletal muscle molecular marker expression levels. Therefore, molecular markers were clustered into processes, and assessed within the context of co-clustered markers. Subsequently, an integrative clustering approach was used for unbiased identification of patient groups with a differential skeletal muscle molecular response to PR.

\section{Patient clusters with differential skeletal muscle molecular rehabilitation responses}

We identified two major patient clusters which differed in both the direction and magnitude of the molecular response to PR. Overall, C1 displayed the most pronounced changes in markers of Autophagy, Myogenesis, Glucocorticoid signaling, Oxidative metabolism regulation, and OXCAP in response to PR.

Specifically, C1 displayed a change in Autophagy-related marker expression reflective of a decrease in autophagy, and a corresponding decrease in Glucocorticoid signalingrelated mRNA expression upon PR, as is in line with the PR-response in previous studies (e.g. [16]). Furthermore, corresponding to previous observations [10, 16, 18], AKT/mTOR signaling was induced upon PR, resulting in an increased capacity and stimulatory signaling of protein synthesis. In keeping with an induction of anabolic signaling upon PR in C1, proliferation marker expression was mostly increased. However, in contrast to previous studies $[10,16]$, mRNA expression of the myogenic differentiation markers MYOD1 and CDH15 were reduced, while mRNA expression of the fusion marker MYMK was induced upon PR in C1. Furthermore, a decrease in expression of 


\section{CHAPTER 5}

Oxidative metabolism regulation markers was observed in C1. Nevertheless, this does not negate the previously reported importance of oxidative metabolism regulation in the coordination of exercise training-induced metabolic alterations [37]. Indeed, as anticipated upon exercise training [38], OXCAP protein expression was increased upon PR in C1. Moreover, the PR-induced modulation of Mitophagy-related markers in C1 is in agreement with an important role for mitochondrial dynamics and mitophagy in the regulation of mitochondrial quality upon exercise training, as reviewed by Drake et al. [37]. Together, these data indicate a relative decrease in catabolic signaling, a relative increase in anabolic signaling, and an increase in mediators of oxidative metabolism upon PR in C1, which strikingly reflects a normal response to exercise training [39]. Importantly, the 'normal' molecular response observed in C1 was accompanied by a strong improvement in physical functioning in this cluster, as determined by an increase in peak load on the cycle ergometer test and an improvement in walking distance in the 6MWT.

In C2, both the functional and molecular responses to PR were less pronounced. This cluster significantly differed from $\mathrm{C} 1$ in the rehabilitation-induced regulation of Autophagy, Myogenesis, Glucocorticoid signaling, and Oxidative metabolism regulation. Specifically, expression of Autophagy-related mRNA and Glucocorticoid signaling markers remained unaltered, or even increased upon PR in C2, which is in line with previous observations $[10,18]$, and may be due to disease-related factors such as hypoxia or systemic inflammation $[40,41]$. However, an early transient induction of catabolic processes is also evident upon exercise, and may play an important role in muscle regeneration [42]. Furthermore, the differential regulation of Myogenesis is reflected by a differential change in the expression of specific markers. Interestingly, the reduction in the Myogenesis markers MYOD1 and CDH15, and induction in MYMK mRNA expression upon PR in C1, was previously shown to be reflective of late-stage muscle regeneration $[43,44]$. Conversely, the apparent increase in MYOD1 and CDH15 mRNA expression, together with only a tendency towards an increase in MYMK upon $P R$ in $C 2$, is reflective of an earlier phase of muscle regeneration [43, 44]. Despite differences in the regulation of Autophagy, Myogenesis, and Glucocorticoid signaling between $\mathrm{C} 1$ and $\mathrm{C} 2$, these processes correlate similarly in both clusters, which shows that there is no cluster-specific dissociation between these processes. This may indicate that, with respect to muscle mass plasticity, both clusters display a normal physiological response to $\mathrm{PR}$, but may reflect distinct phases of PR-induced muscle remodeling. 
Interestingly, we found a tendency towards a differential association between Oxidative metabolism regulation and OXCAP in Clusters 1 and 2, and a differential correlation between protein turnover and mitochondrial turnover regulatory processes. This supports previous studies showing a correlation between tissue remodeling and bioenergetics pathways that were altered in COPD [36, 43, 45], and adds that this alteration may be present only in a subgroup of patients. Indeed, as expected upon exercise training [39], C1 displayed an increase in OXCAP markers upon $\mathrm{PR}$, reflecting a restoration of the oxidative metabolic machinery. This is corroborated by the decreased expression of Oxidative metabolism regulation markers, as a normalization of metabolism-related mRNA has previously been shown in late-stage muscle regeneration $[43,46]$. Conversely, in C2, Oxidative metabolism regulation and OXCAP were statistically unaltered, although Oxidative metabolism regulation seemed to increase upon PR. Furthermore, C2 displayed a strong induction in PINK1 mRNA expression, which plays an important role in the regulation of mitochondrial quality [47]. Together, this suggests that the oxidative metabolic machinery is incompletely restored in C2, which may indicate that this process is impaired-as previously suggested in COPD [48]—or yet ongoing.

\section{Potential drivers of cluster differences}

Compellingly, and in line with previous studies (e.g. $[6,7,9,22,49])$, baseline variables did not seem predictive of the muscle molecular or functional response, as patient clusters did not differ in baseline values of functional parameters, demographics, pulmonary function, body composition, presence of comorbidities, or use of medication. Furthermore, both the current physiological and molecular data do not provide clear indications of a 'defective' response in either cluster. Rather, we propose that molecular differences between clusters are reflective of distinct phases of muscle remodeling, which may be due to differences in the speed by which the remodeling process occurs and/or the timing of initiation of muscle remodeling-related processes upon PR. Interestingly, a differential response to PR may reflect the underlying cause of exercise limitation [50], including reduced muscle capillarization and neuromuscular function as reported upon aging [11, 12], which delays or slows PR-induced muscle mass and metabolic remodeling processes. This may be influenced by lifestyle factors, which is corroborated by the tendency towards a difference in smoking status and blood lipid profile between the clusters. Alternatively, the molecular changes reflecting an early stage of muscle remodeling may originate from an impaired progression through regulating processes, preventing their late-stage normalization, as may be the case for Myogenesis [51]. However, the drivers of differential clustering remain elusive, and will be an important target for future investigations. 


\section{CHAPTER 5}

\section{Strengths and limitations}

We acknowledge that future longitudinal studies are needed to discern a potential defect in muscle mass and metabolic plasticity from a differential progression of these processes. Nevertheless, the use of a relatively short intervention period was likely critical in the identification of clusters with molecular differences reflective of distinct phases of the muscle remodeling process.

As we aimed to address the variability in the skeletal muscle rehabilitation response between COPD patients, no healthy control group was included. Furthermore, although no PR-induced change in FFMI was detected by BIA, we did not measure changes in muscle mass using a sensitive measure such as CT or MRI. Therefore, we cannot confirm our assumption that $\mathrm{C} 1$ displays a more 'healthy' response to PR than C2. However, it is unlikely that the delivered training load differed between clusters, as the training program was conducted in a controlled, supervised in-patient setting. Moreover, the training modality was based on baseline characteristics, which did not differ between clusters.

The current study population was characterized by severe COPD and a high prevalence of muscle mass depletion. Therefore, distribution of patients among clusters cannot be directly extrapolated to the general COPD population. In line, it should be noted that aside from the two extensively discussed major clusters, two other patient clusters have been identified. We chose to omit these two very small clusters in further analysis, rather than recursively merging clusters based on their hierarchical structure, since they showed a distinct molecular response to PR. However, these clusters may thus be clinically meaningful, and call for future investigation.

To support identification of robust, clinically relevant subgroups, patients were clustered based on process scores. The physiological relevance of the identified processes is substantiated by the separation of two mitophagy processes that have been reported in literature [52], and was achieved by inclusion of prior knowledge of the molecular network into the marker clustering [53]. To this end, we relied on well-established knowledge of their interactions, and previous unbiased network analyses of 'omics' data [36, 45]. In contrast to these studies, we have performed a targeted analysis of a selection of molecular markers, which can be confined in future studies as several markers show a low contribution to the process scores. Nevertheless, component loadings did not imply single biomarkers for the identified processes. Moreover, assessment of single marker expression proved important for the interpretation of observed differences in processes, certainly in the context of temporal 
responses, and therefore remains necessary for obtaining insight in the mechanisms underlying muscle dysfunction in COPD.

\section{Conclusion}

Based on the cluster-based analysis, no specific cluster with 'non-responders' was identified, reinforcing that exercise training should form a cornerstone of PR to improve skeletal muscle function in advanced COPD. However, this study identifies two major clusters of COPD patients with a differential muscle molecular rehabilitation response, which may be reflective of distinct phases of muscle remodeling, and corresponds to a differential gain in physical functioning.

Identification of the drivers of these differential temporal responses will provide an opportunity for personalized interventions optimizing the exercise training response. We propose that, next to detailed analyses of baseline lifestyle factors such as smoking, diet and physical activity, and lung function impairments and blood gasses, future studies should investigate muscle intrinsic differences that determine the kinetics of the muscle molecular response to PR. This could open up new avenues for pharmacological and nutritional interventions to optimize the skeletal muscle functional improvements upon PR. 


\section{CHAPTER 5}

\section{REFERENCES}

1. Barnes PJ, Celli BR. Systemic manifestations and comorbidities of COPD. Eur Respir J 2009;33(5):1165-85.

2. Maltais F, Decramer M, Casaburi R, et al. An official American Thoracic Society/European Respiratory Society statement: update on limb muscle dysfunction in chronic obstructive pulmonary disease. Am J Respir Crit Care Med 2014;189(9):e15-62.

3. Mostert R, Goris A, Weling-Scheepers $\mathrm{C}$, et al. Tissue depletion and health related quality of life in patients with chronic obstructive pulmonary disease. Respir Med 2000;94(9):859-67.

4. Marquis $\mathrm{K}$, Debigare $\mathrm{R}$, Lacasse $\mathrm{Y}$, et al. Midthigh muscle cross-sectional area is a better predictor of mortality than body mass index in patients with chronic obstructive pulmonary disease. Am J Respir Crit Care Med 2002;166(6):809-13.

5. Schols AM, Broekhuizen R, Weling-Scheepers CA, et al. Body composition and mortality in chronic obstructive pulmonary disease. Am J Clin Nutr 2005;82(1):53-9.

6. Troosters $T$, Gosselink R, Decramer M. Exercise training in COPD: how to distinguish responders from nonresponders. J Cardiopulm Rehabil 2001;21(1):10-7.

7. Garrod R, Marshall J, Barley E, et al. Predictors of success and failure in pulmonary rehabilitation. Eur Respir J 2006;27(4):788-94.

8. Spruit MA, Augustin IM, Vanfleteren LE, et al. Differential response to pulmonary rehabilitation in COPD: multidimensional profiling. Eur Respir J 2015;46(6):1625-35.

9. Altenburg $\mathrm{WA}$, de Greef $\mathrm{MH}$, ten Hacken $\mathrm{NH}$, et al. A better response in exercise capacity after pulmonary rehabilitation in more severe COPD patients. Respir Med 2012;106(5):694700.

10. Constantin D, Menon MK, Houchen-Wolloff $L$, et al. Skeletal muscle molecular responses to resistance training and dietary supplementation in COPD. Thorax 2013;68(7):625-33.

11. Joanisse $S$, Nederveen JP, Snijders $T$, et al. Skeletal Muscle Regeneration, Repair and Remodelling in Aging: The Importance of Muscle Stem Cells and Vascularization. Gerontology 2017;63(1):91-100.

12. Baehr LM, West DW, Marcotte G, et al. Age-related deficits in skeletal muscle recovery following disuse are associated with neuromuscular junction instability and ER stress, not impaired protein synthesis. Aging (Albany NY) 2016;8(1):127-46.

13. Shad BJ, Thompson JL, Breen L. Does the muscle protein synthetic response to exercise and amino acid-based nutrition diminish with advancing age? A systematic review. Am J Physiol Endocrinol Metab 2016;311(5):E803-E17.

14. Theriault ME, Pare ME, Lemire BB, et al. Regenerative defect in vastus lateralis muscle of patients with chronic obstructive pulmonary disease. Respir Res 2014;15:35.

15. Kneppers AEM, Langen RCJ, Gosker HR, et al. Increased Myogenic and Protein Turnover Signaling in Skeletal Muscle of Chronic Obstructive Pulmonary Disease Patients With Sarcopenia. J Am Med Dir Assoc 2017;18(7):637 e1-37 e11.

16. Vogiatzis I, Simoes DC, Stratakos G, et al. Effect of pulmonary rehabilitation on muscle remodelling in cachectic patients with COPD. Eur Respir J 2010;36(2):301-10.

17. Vogiatzis I, Terzis G, Stratakos G, et al. Effect of pulmonary rehabilitation on peripheral muscle fiber remodeling in patients with COPD in GOLD stages II to IV. Chest 2011;140(3):744-52.

18. Costes $F$, Gosker $H$, Feasson $L$, et al. Impaired exercise training-induced muscle fiber hypertrophy and Akt/mTOR pathway activation in hypoxemic patients with COPD. Journal of applied physiology 2015;118(8):1040-9.

19. Crisafulli E, Costi $S$, Luppi $F$, et al. Role of comorbidities in a cohort of patients with COPD undergoing pulmonary rehabilitation. Thorax 2008;63(6):487-92. 
20. Walsh JR, McKeough ZJ, Morris NR, et al. Metabolic disease and participant age are independent predictors of response to pulmonary rehabilitation. J Cardiopulm Rehabil Prev 2013;33(4):249-56.

21. Evans RA, Singh SJ, Collier R, et al. Pulmonary rehabilitation is successful for COPD irrespective of MRC dyspnoea grade. Respir Med 2009;103(7):1070-5.

22. Jones SE, Maddocks M, Kon SS, et al. Sarcopenia in COPD: prevalence, clinical correlates and response to pulmonary rehabilitation. Thorax 2015;70(3):213-8.

23. Cebron Lipovec N, Schols AM, van den Borst B, et al. Sarcopenia in Advanced COPD Affects Cardiometabolic Risk Reduction by Short-Term High-intensity Pulmonary Rehabilitation. J Am Med Dir Assoc 2016;17(9):814-20.

24. Miller MR, Crapo R, Hankinson J, et al. General considerations for lung function testing. Eur Respir J 2005;26(1):153-61.

25. Laboratories ATSCoPSfCPF. ATS statement: guidelines for the six-minute walk test. Am J Respir Crit Care Med 2002;166(1):111-7.

26. Franssen FM, Sauerwein HP, Ackermans MT, et al. Increased postabsorptive and exerciseinduced whole-body glucose production in patients with chronic obstructive pulmonary disease. Metabolism 2011;60(7):957-64.

27. Holland $A E$, Hill CJ, Rasekaba $T$, et al. Updating the minimal important difference for sixminute walk distance in patients with chronic obstructive pulmonary disease. Arch Phys Med Rehabil 2010;91(2):221-5.[published Online First: 2010/02/18]

28. Cazzola M, MacNee W, Martinez FJ, et al. Outcomes for COPD pharmacological trials: from lung function to biomarkers. Eur Respir J 2008;31(2):416-69.

29. Newman AB, Kupelian V, Visser M, et al. Sarcopenia: alternative definitions and associations with lower extremity function. J Am Geriatr Soc 2003;51(11):1602-9.

30. Vestbo J, Hurd SS, Agusti AG, et al. Global strategy for the diagnosis, management, and prevention of chronic obstructive pulmonary disease: GOLD executive summary. Am J Respir Crit Care Med 2013;187(4):347-65.

31. Spruit MA, Singh SJ, Garvey C, et al. An official American Thoracic Society/European Respiratory Society statement: key concepts and advances in pulmonary rehabilitation. Am J Respir Crit Care Med 2013;188(8):e13-64.

32. McCarthy B, Casey D, Devane $D$, et al. Pulmonary rehabilitation for chronic obstructive pulmonary disease. Cochrane Database Syst Rev 2015(2):CD003793.

33. Snijders T, Nederveen JP, McKay BR, et al. Satellite cells in human skeletal muscle plasticity. Frontiers in physiology 2015;6:283.

34. Sanchez AM, Bernardi H, Py G, et al. Autophagy is essential to support skeletal muscle plasticity in response to endurance exercise. Am J Physiol Regul Integr Comp Physiol 2014;307(8):R956-69.

35. Vainshtein A, Tryon LD, Pauly M, et al. Role of PGC-1alpha during acute exercise-induced autophagy and mitophagy in skeletal muscle. Am J Physiol Cell Physiol 2015;308(9):C710-9.

36. Turan N, Kalko S, Stincone A, et al. A systems biology approach identifies molecular networks defining skeletal muscle abnormalities in chronic obstructive pulmonary disease. PLoS Comput Biol 2011;7(9):e1002129.

37. Drake JC, Wilson RJ, Yan Z. Molecular mechanisms for mitochondrial adaptation to exercise training in skeletal muscle. FASEB journal : official publication of the Federation of American Societies for Experimental Biology 2016;30(1):13-22.

38. MacMillan NJ, Kapchinsky S, Konokhova Y, et al. Eccentric Ergometer Training Promotes Locomotor Muscle Strength but Not Mitochondrial Adaptation in Patients with Severe Chronic Obstructive Pulmonary Disease. Frontiers in physiology 2017;8:114.

39. Egan B, Zierath JR. Exercise metabolism and the molecular regulation of skeletal muscle 


\section{CHAPTER 5}

adaptation. Cell Metab 2013;17(2):162-84.

40. de Theije CC, Schols A, Lamers WH, et al. Glucocorticoid Receptor Signaling Impairs Protein Turnover Regulation in Hypoxia-Induced Muscle Atrophy in Male Mice. Endocrinology 2018;159(1):519-34.

41. Ceelen JJ, Langen RC, Schols AM. Systemic inflammation in chronic obstructive pulmonary disease and lung cancer: common driver of pulmonary cachexia? Curr Opin Support Palliat Care 2014;8(4):339-45.

42. Tipton KD, Hamilton DL, Gallagher IJ. Assessing the Role of Muscle Protein Breakdown in Response to Nutrition and Exercise in Humans. Sports Med 2018;48(Suppl 1):53-64.

43. Aguilar CA, Shcherbina A, Ricke DO, et al. In vivo Monitoring of Transcriptional Dynamics After Lower-Limb Muscle Injury Enables Quantitative Classification of Healing. Sci Rep 2015;5:13885.

44. Pansters NA, Schols AM, Verhees KJ, et al. Muscle-specific GSK-3beta ablation accelerates regeneration of disuse-atrophied skeletal muscle. Biochim Biophys Acta 2015;1852(3):490506.

45. Tenyi A, Cano I, Marabita F, et al. Network modules uncover mechanisms of skeletal muscle dysfunction in COPD patients. J Transl Med 2018;16(1):34.

46. Remels AH, Pansters NA, Gosker HR, et al. Activation of alternative NF-kappaB signaling during recovery of disuse-induced loss of muscle oxidative phenotype. Am J Physiol Endocrinol Metab 2014;306(6):E615-26.

47. Narendra D, Walker JE, Youle R. Mitochondrial quality control mediated by PINK1 and Parkin: links to parkinsonism. Cold Spring Harb Perspect Biol 2012;4(11)

48. Marin de Mas I, Fanchon E, Papp B, et al. Molecular mechanisms underlying COPD-muscle dysfunction unveiled through a systems medicine approach. Bioinformatics 2017;33(1):95103.

49. Mesquita R, Vanfleteren LE, Franssen FM, et al. Objectively identified comorbidities in COPD: impact on pulmonary rehabilitation outcomes. Eur Respir J 2015;46(2):545-8.

50. Plankeel JF, McMullen B, Maclntyre NR. Exercise outcomes after pulmonary rehabilitation depend on the initial mechanism of exercise limitation among non-oxygen-dependent COPD patients. Chest 2005;127(1):110-6.

51. Talbert $\mathrm{EE}$, Guttridge DC. Impaired regeneration: A role for the muscle microenvironment in cancer cachexia. Semin Cell Dev Biol 2016;54:82-91.

52. Palikaras K, Lionaki E, Tavernarakis N. Mechanisms of mitophagy in cellular homeostasis, physiology and pathology. Nat Cell Biol 2018;20(9):1013-22.

53. Reshetova P, Smilde AK, van Kampen AH, et al. Use of prior knowledge for the analysis of high-throughput transcriptomics and metabolomics data. BMC Syst Biol 2014;8 Suppl 2:S2.

54. von Haehling S, Morley JE, Coats AJ, et al. Ethical guidelines for publishing in the Journal of Cachexia, Sarcopenia and Muscle: update 2015. J Cachexia Sarcopenia Muscle 2015;6(4):3156. 


\section{SUPPLEMENTARY MATERIAL}

\section{METHODS}

\section{$R T-q P C R$}

Tissue was homogenized (Beat-Beater) in presence of TRI-reagent (Sigma), and RNA was isolated byTRI-reagent/Chloroform extraction and subsequently precipitated from the aqueous phase using glycogen-containing isopropanol. RNA was reconstituted in $30 \mu \mathrm{l}$ RNA storage solution (Ambion AM7001) and stored at $-80^{\circ} \mathrm{C}$. RNA concentrations were measured spectrophotometrically using a Nanodrop UV-Vis spectrophotometer (ND-1000, Thermo Scientific). 400 ng RNA was diluted in nuclease free $\mathrm{H}_{2} \mathrm{O}$ and reverse transcribed to cDNA using the Tetro cDNA synthesis kit (Bioline) according to the manufacturer's instructions. qPCR primers were designed based on Ensembl transcript sequences and ordered from Sigma Genosys (Zwijndrecht, the Netherlands), with primer details shown in Table A. qPCR reactions contained Sensimix SYBR \& ROX (GC Biotech) and primer mix, and were run in a 384 well white opaque plate (Roche) on a LightCycler 480 system (Roche). Melting curves were analysed to verify specificity of the amplification, and relative quantity of the targets was assessed by LinRegPCR software (v2014.8) [1]. Three reference genes (RPLP0, B2M, and PPIA) were used to calculate a GeNorm correction factor, which was used to normalize expression levels of the target genes.

\section{Western Blot}

Tissue was homogenized in $600 \mu$ of Immunoprecipitation lysis buffer (50mM Tris, $150 \mathrm{mM} \mathrm{NaCl}, 10 \%$ glycerol, $0.5 \%$ Nonidet P40, protease and phosphatase inhibitors (Roche)) with a Micro Tissue Homogenizer. After homogenization, samples were incubated on a tube rotator at $4^{\circ} \mathrm{C}$ for 30 minutes and centrifuged at $14000 \mathrm{~g}$ at $4^{\circ} \mathrm{C}$ for 30 minutes. The supernatant was stored at $-80^{\circ} \mathrm{C}$ until analysis. Total protein concentration was determined using BCA Protein Assay kit (Pierce) according to the manufacturer's instructions. For Western Blot analyses, 4x Laemmli sample buffer (0.25M Tris-HCl ph6.8; 8\% (w/v) SDS; 40\% (v/v) glycerol; $0.4 \mathrm{M} \mathrm{DTT}$ and $0.02 \%(\mathrm{w} / \mathrm{v})$ Bromophenol Blue) was added and samples were heated to $100^{\circ} \mathrm{C}$ for 5 minutes. 10 $\mu \mathrm{g}$ protein was separated on a Criterion XT Precast 4-12\% Bis-Tris gel (Bio-Rad) in XT MOPS running buffer (Bio-Rad) by gel electrophoresis. Proteins were transferred to a nitrocellulose membrane (Bio-Rad) by electroblotting at $100 \mathrm{~V}$ for 60 minutes in transfer buffer (25 mM Tris, 192 mM Glycine, 20\% (vol/vol) methanol). 


\section{CHAPTER 5}

For total protein detection, the membrane was stained with PonceauS solution $(0.2 \%$ PonceauS in 1\% acetic acid; Sigma-Aldrich Chemie) and imaged using the Amersham imager 600RGB. The membrane was blocked for 1 hour at room temperature (RT) in Tris-buffered saline with Tween20 (TBST; $20 \mathrm{mM}$ Tris, $137 \mathrm{mM} \mathrm{NaCl}, 0.1 \%$ (vol/vol) Tween20, pH 7.6) containing 3\% (w/v) nonfat dry milk (Campina, Eindhoven, the Netherlands). The membranes were washed in TBST, followed by overnight incubation at $4^{\circ} \mathrm{C}$ with primary antibody diluted in TBST with $3 \%$ BSA or non-fat dry milk (Table B). Membranes were incubated with a peroxidase conjugated secondary antibody solution (Vector Laboratories, \#PI-1000 (Diluted 1:10000 in blocking solution)) for 1 hour at RT, and targets were visualized by chemiluminescence using Supersignal West PICO or FEMTO Chemiluminescent Substrate (Pierce Biotechnology, Inc.) according to the manufacturer's instructions, and detected using the Amersham imager 600RGB. Signals were quantified with Image Quant software (Amersham). For analyses, samples from controls and COPD patients were randomly distributed within and between blots, and reference samples were loaded onto all blots to correct for between-blot variation. Protein expression and phosphorylation levels were corrected for total protein content, and presented as a fold change compared to the control group.

\section{Data analysis}

\section{Clustering}

Molecular rehabilitation responses were calculated as the percentage change from pre-rehabilitation values. Before clustering, data were pre-processed per molecular marker by (i) imputing missing values based on the median value across patients, (ii) removing outliers by truncating at the 3-97 percentile interval and (iii) scaling the data between 0 and 1 .

- First, network-constrained agglomerative clustering of the molecular markers using Ward's criteria [2] was performed as implemented in the scikit-learn (version 19.0) environment [3]. This recursively merges the pair of clusters that minimally increases within-cluster variance. Defaults settings were used, except that a literature-based molecular network was used to define a sparse connectivity matrix. As such, clustering of molecular markers with known functional interactions was prioritized. A reasonable number of marker clusters (i.e., processes) was chosen based on the cluster size, and physiological relevance. Graph-tool (version 2.26) was used to visualize the network of molecular markers and subsequent clustering result [4]. Second, data dimensionality was reduced by computing a single process score for each process based on the associated individual marker values by principle component analysis (PCA) within scikit- 
learn. To assess the contribution of individual markers to the process scores, component loadings were obtained using the 'components_' attibute provided by scikit-learn's PCA module.

- Processes were named based on enrichment of Gene Ontology (GO) biological processes, as determined by Panther ((http://www.pantherdb.org/, version 13.0) statistical overrepresentation test (Fisher's exact test with FDR $<0.02)[5,6]$. When no significant overrepresentation of GO-terms was detected, the process name was based on the markers' predominant muscle-related GO annotations.

As for the molecular markers, patients were hierarchically clustered using Ward's criteria based on their process scores, however, without connectivity prior. This was repeated across a range of predefined number of clusters (i.e., 2-10), after which the optimal number of clusters was chosen based on the 'silhouette' score [7]. The silhouette score was calculated using the corresponding module within scikit-learn.

\section{REFERENCES}

1. Ramakers C, Ruijter JM, Deprez RH, et al. Assumption-free analysis of quantitative real-time polymerase chain reaction (PCR) data. Neurosci Lett 2003;339(1):62-6.

2. Ward JH. Hierarchical Grouping to Optimize an Objective Function. Journal of the American Statistical Association 1963;58(301):236-44.

3. Pedregosa F, Ga, \#235, et al. Scikit-learn: Machine Learning in Python. J Mach Learn Res 2011;12:2825-30.

4. The graph-tool python library [program], 2017.

5. Mi H, Huang X, Muruganujan A, et al. PANTHER version 11: expanded annotation data from Gene Ontology and Reactome pathways, and data analysis tool enhancements. Nucleic Acids Res 2017;45(D1):D183-D89.

6. The Gene Ontology C. Expansion of the Gene Ontology knowledgebase and resources. Nucleic Acids Res 2017;45(D1):D331-D38.

7. Rousseeuw PJ. Silhouettes: A graphical aid to the interpretation and validation of cluster analysis. Journal of Computational and Applied Mathematics 1987;20(Supplement C):5365. 


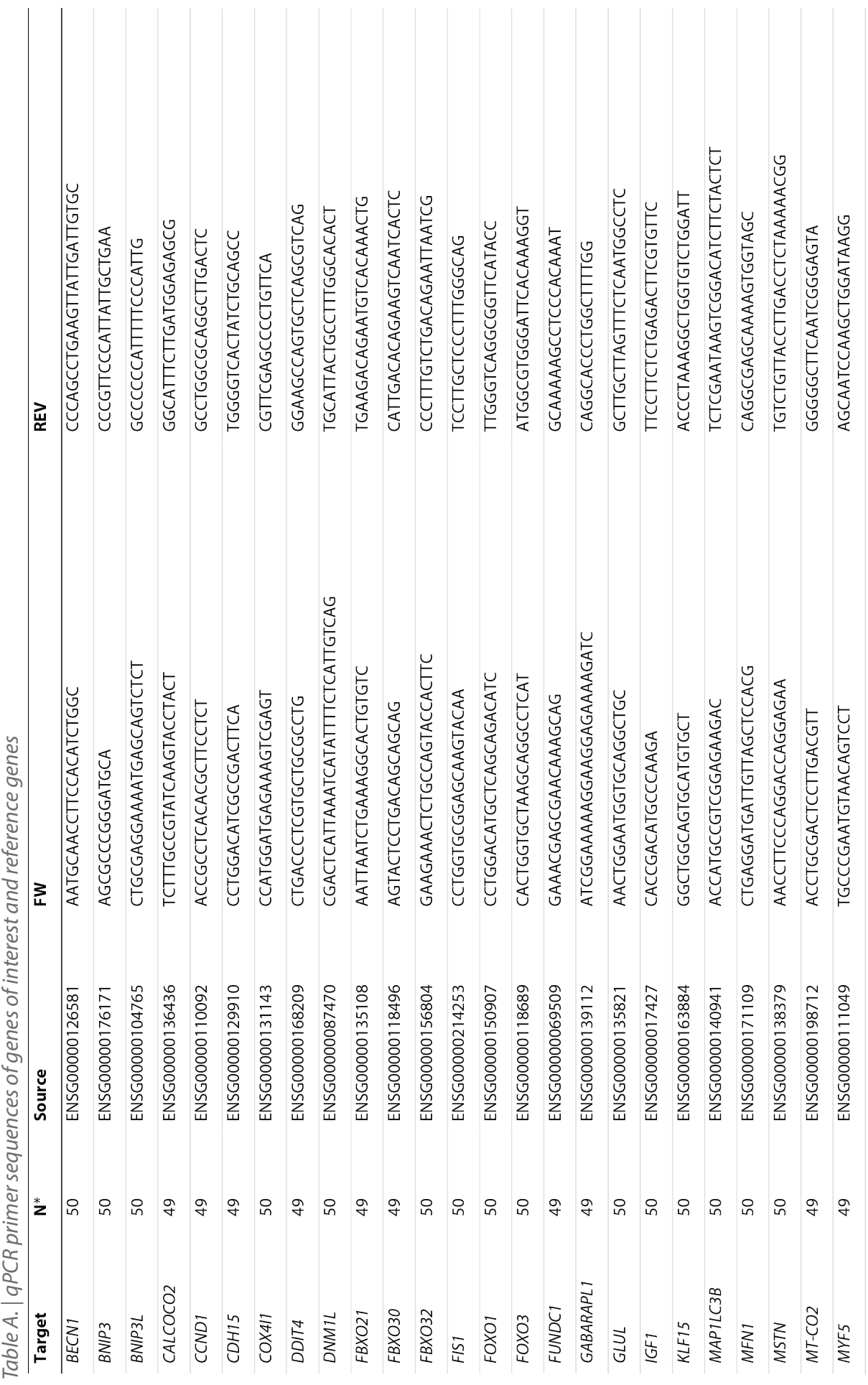



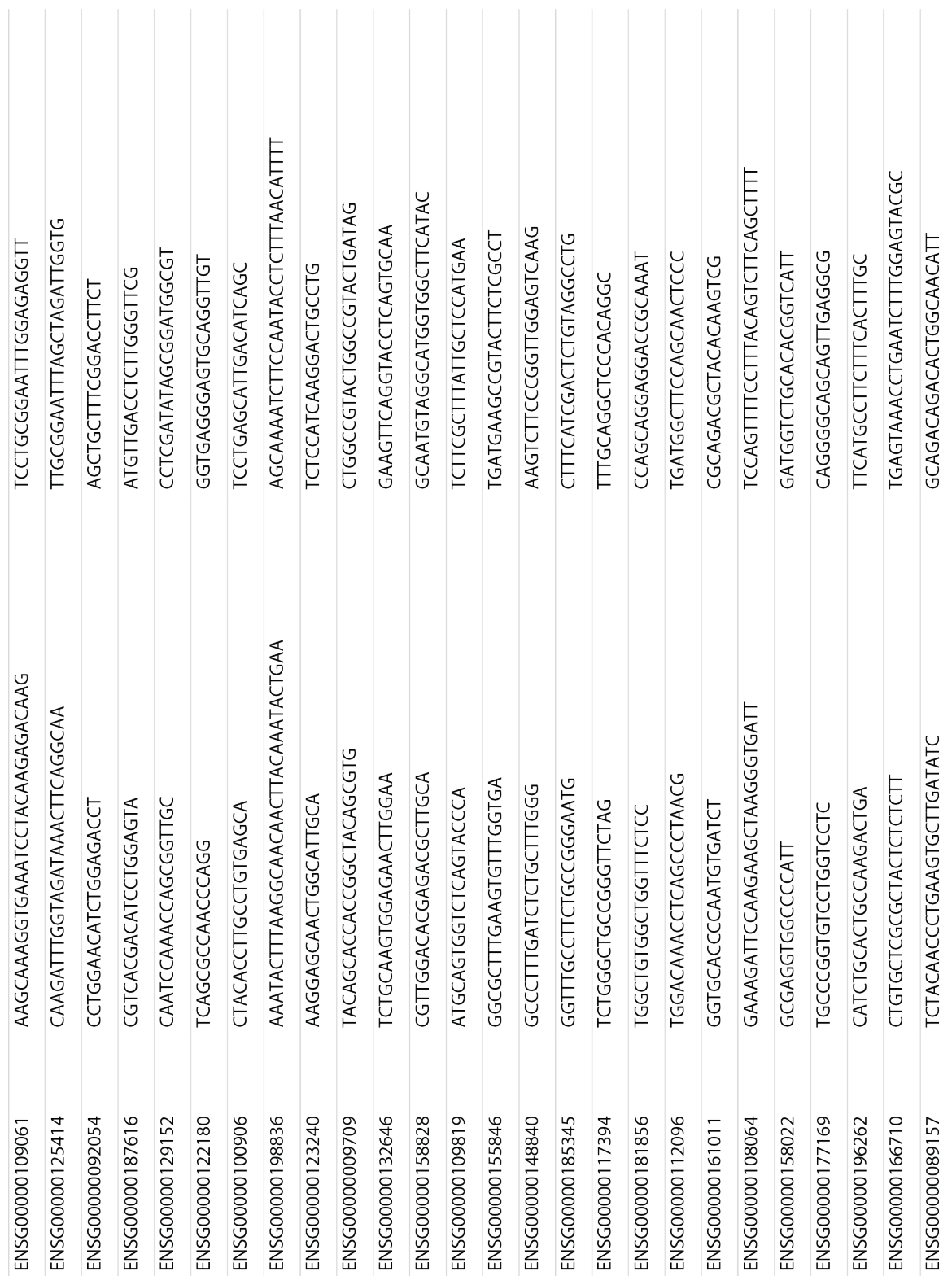

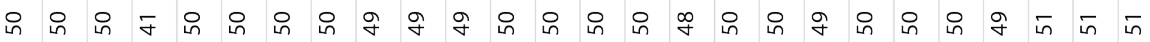

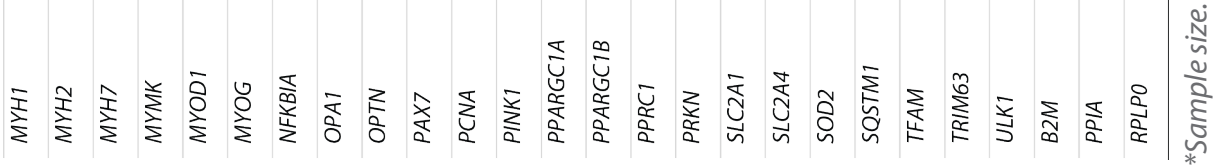




\section{CHAPTER 5}

Table B. | Primary antibody list

\begin{tabular}{|c|c|c|c|}
\hline Target & $\mathbf{N}^{*}$ & Supplier & Product nr. \\
\hline AKT1 & 51 & Cell signaling & 9272 \\
\hline AKT1 (Ser473) & 51 & Cell signaling & 9271 \\
\hline AKT1 (Ser308) & 51 & Cell signaling & 4056 \\
\hline BECN1 & 46 & Novus Biologicals & NB110-87318SS \\
\hline BNIP3L & 46 & Cell signaling & 12396 \\
\hline DNM1L & 46 & Cell signaling & 8570 \\
\hline EIF4EBP1 & 51 & Cell signaling & 9452 \\
\hline ElF4EBP1 (Ser65) & 51 & Cell signaling & 9451 \\
\hline FOXO1 & 51 & Cell signaling & 2880 \\
\hline FOXO3 & 51 & Cell signaling & 2497 \\
\hline FOXO1 (Thr24)/FOXO3 (Thr32) & 51 & Cell signaling & 9464 \\
\hline FUNDC1 & 46 & Santa Cruz & Sc-133597 \\
\hline GSK3B & 51 & Cell signaling & 12456 \\
\hline GSK3B (Ser9) & 51 & Cell signaling & 9336 \\
\hline GYS1 (Ser641) & 51 & Cell signaling & 3891 \\
\hline IRS1 & 50 & Merck Millipore & $06-248$ \\
\hline IRS1(Ser612) & 51 & BIOKÉ & 3203 \\
\hline IRS1(Tyr632) & 50 & Abcam & Ab109543 \\
\hline MAP1LC3B & 45 & Cell signaling & 2775 \\
\hline MTOR & 51 & Cell signaling & 2983 \\
\hline MTOR (Ser2448) & 51 & Cell signaling & 2971 \\
\hline OXPHOS complexes & 51 & MitoSciences & MS604 \\
\hline PRKN & 46 & Cell signaling & 4211 \\
\hline RPS6 & 51 & Cell signaling & 2317 \\
\hline RPS6 (Ser235/236) & 51 & Cell signaling & 4858 \\
\hline SQSTM1 & 46 & Cell signaling & 5114 \\
\hline TBC1D1 & 51 & BIOKÉ & $2670 \mathrm{~S}$ \\
\hline TBC1D1 (Thr642) & 51 & BIOKÉ & $8881 \mathrm{~S}$ \\
\hline TOMM20 & 46 & Abcam & Ab186734 \\
\hline ULK1 & 46 & Cell signaling & 8054 \\
\hline ULK1 (Ser555) & 46 & Cell signaling & 5869 \\
\hline ULK1 (Ser757) & 46 & Cell signaling & 6888 \\
\hline
\end{tabular}

*Sample size. 
A

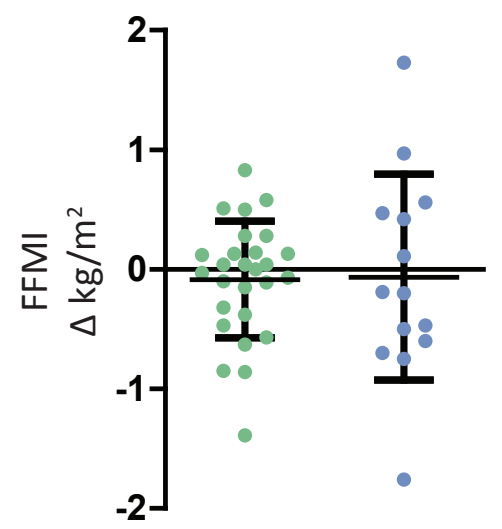

B

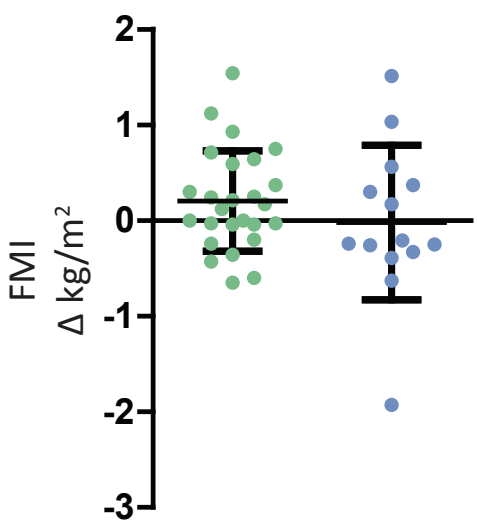

Cluster 1

Cluster 2

Figure S1. |PR-induced changes in body composition. a) PR-induced change in Fat-Free Mass Index (FFMI) in $\mathrm{kg} / \mathrm{m}^{2}$ assessed by BIA. b) PR-induced change in Fat Mass Index (FMI) in $\mathrm{kg} / \mathrm{m}^{2}$ assessed by $B I A$. Data expressed as mean $\pm S D$. 


\section{CHAPTER 5}

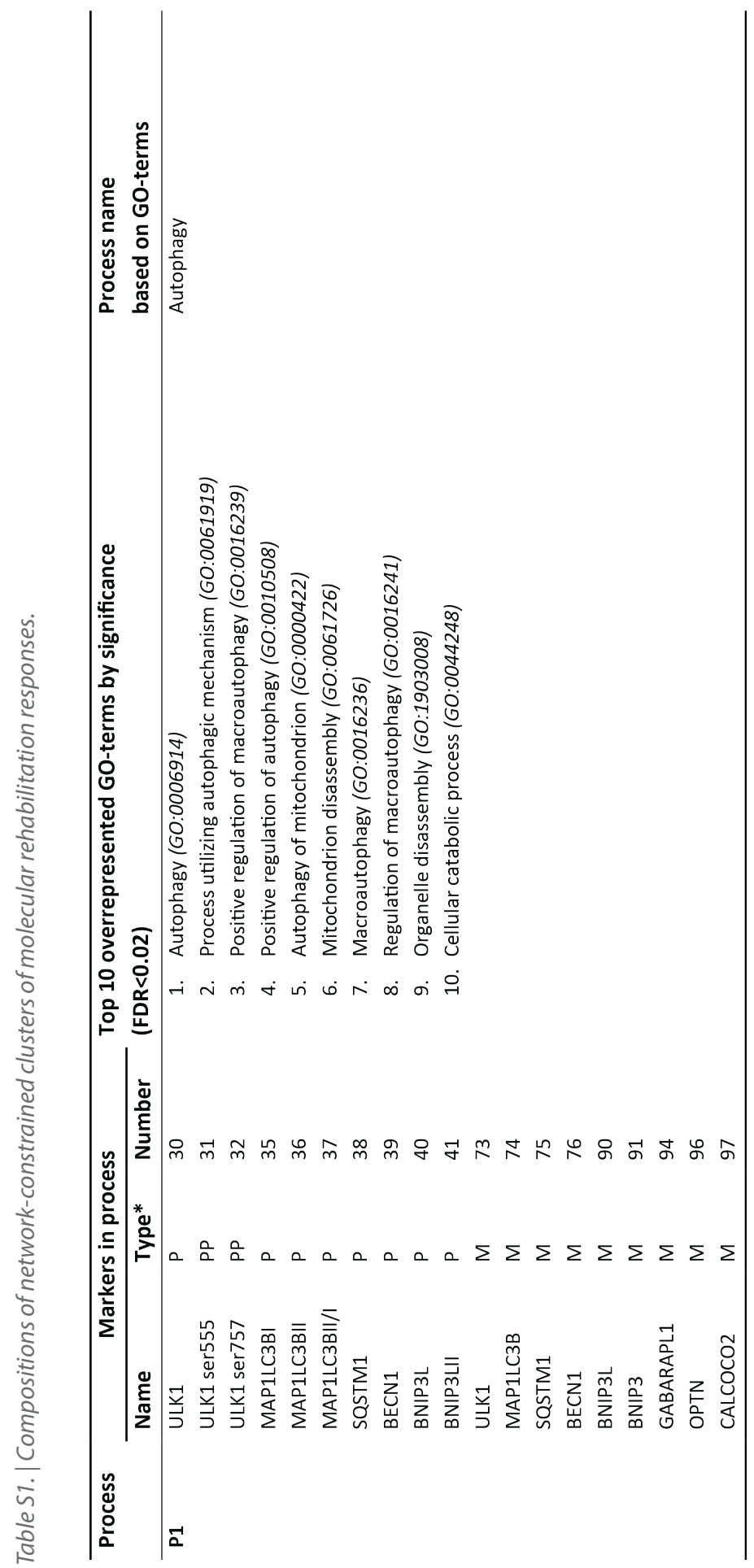




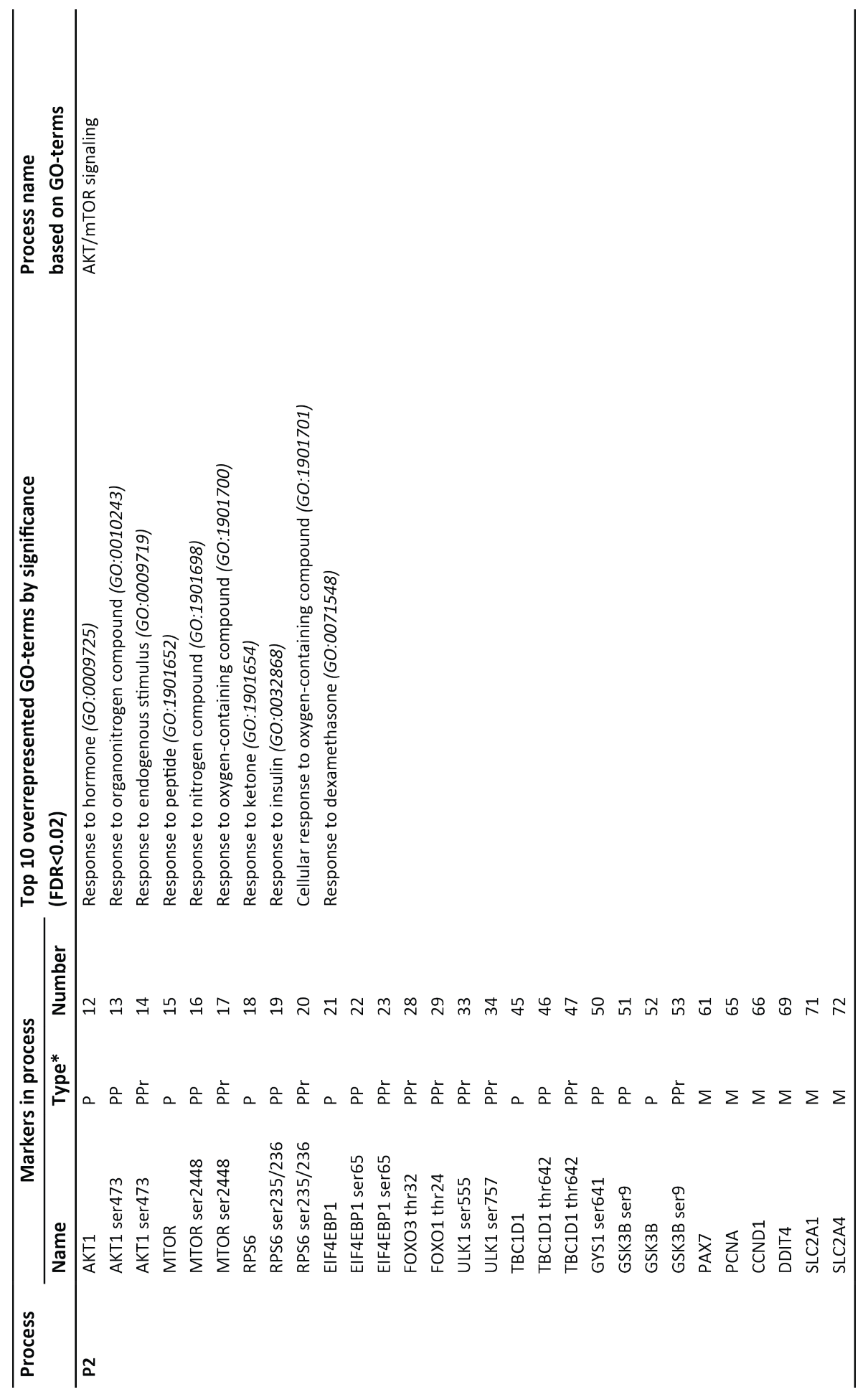




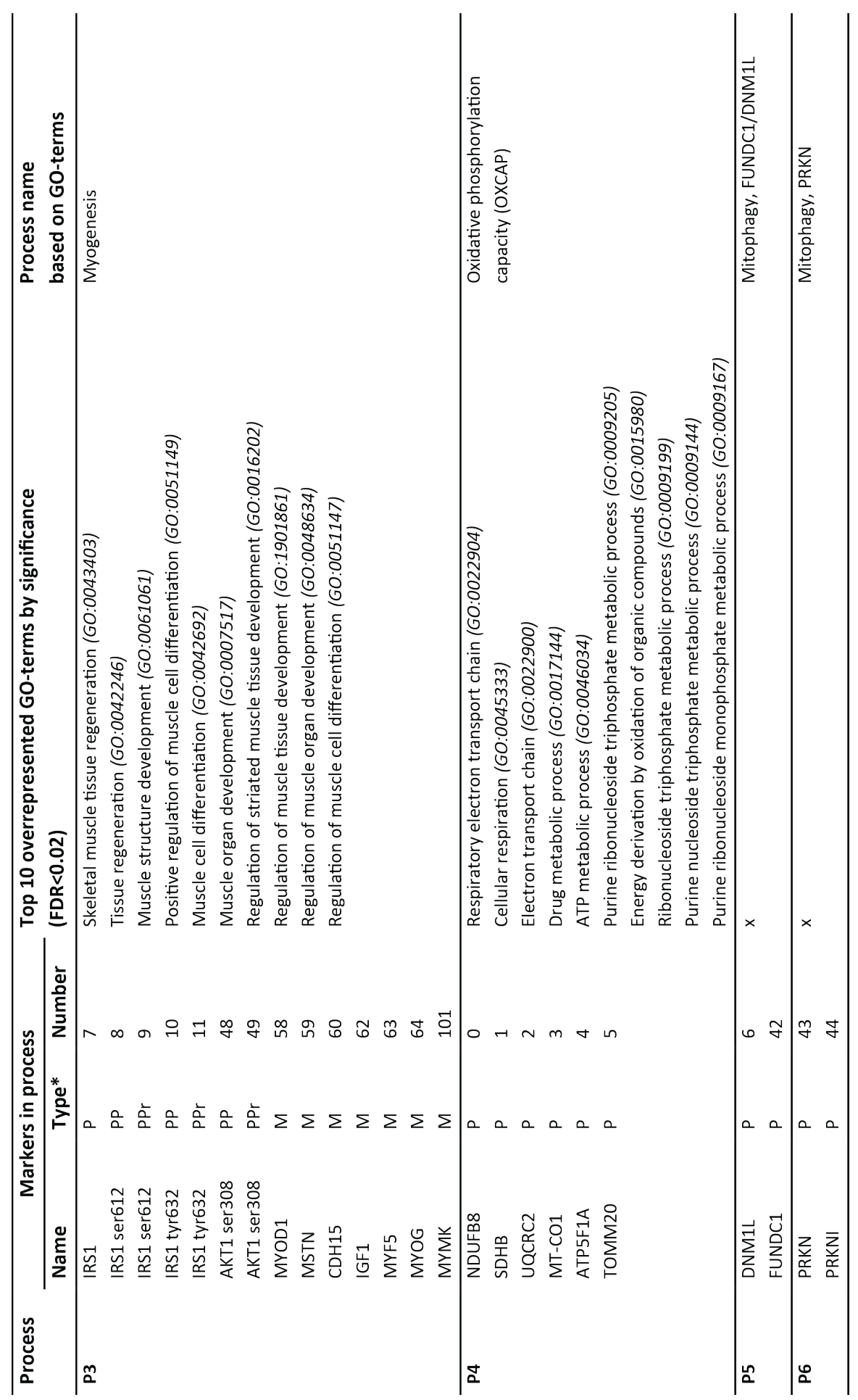




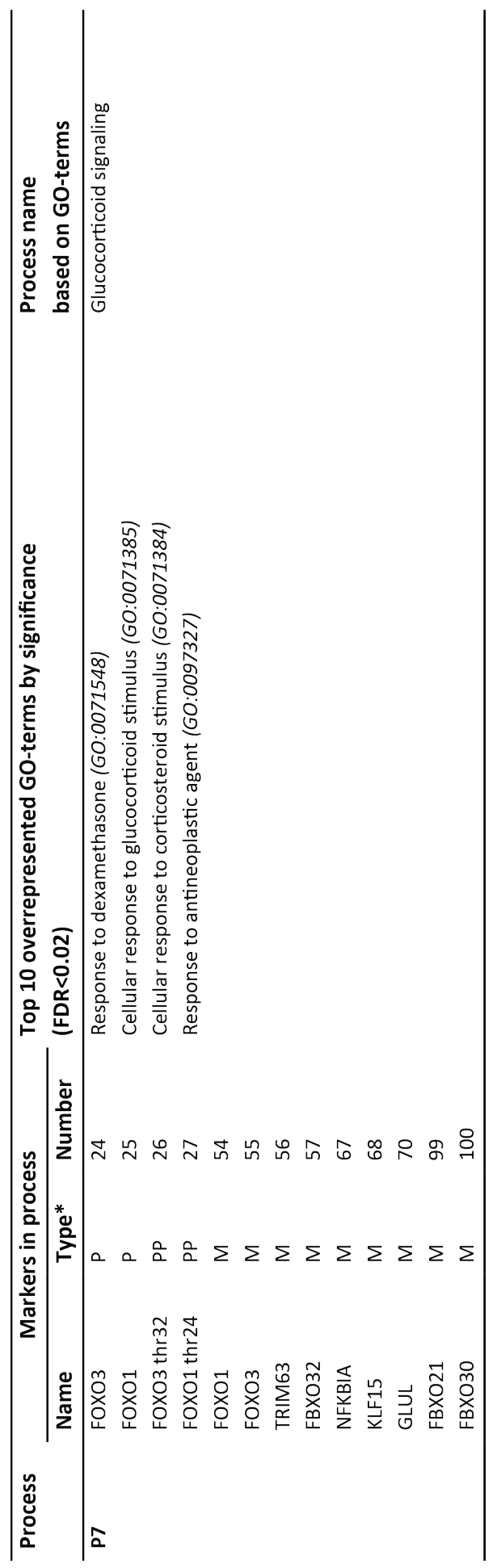




\section{CHAPTER 5}

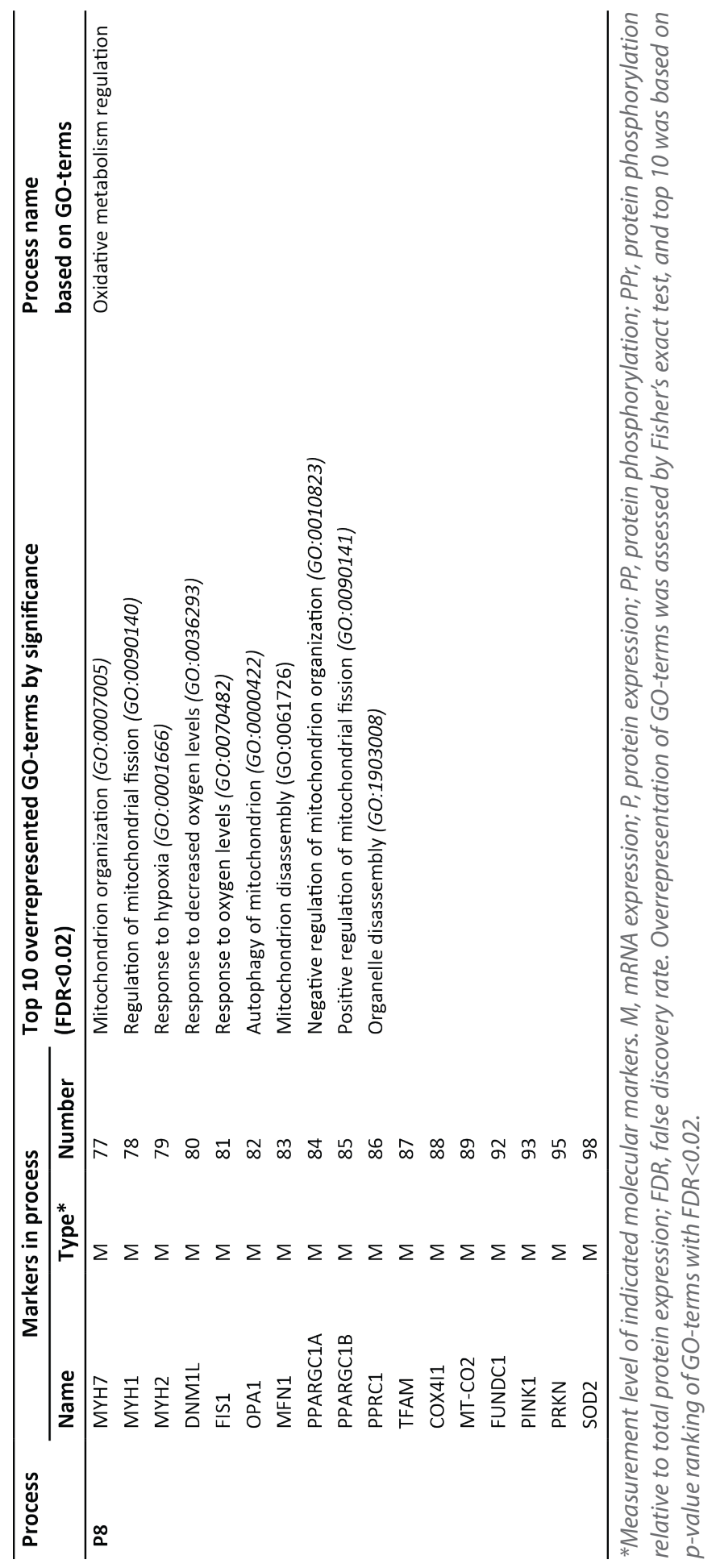


Table S2. | Component loadings of markers on each process

\begin{tabular}{|c|c|c|c|c|c|}
\hline Process & Name & Type* & Number & $\begin{array}{l}\text { Component } \\
\text { loadings }\end{array}$ & $\begin{array}{l}\text { Squared component } \\
\text { loadings }\end{array}$ \\
\hline \multirow[t]{19}{*}{ P1 } & SQSTM1 & $M$ & 75 & -0.41 & 0.17 \\
\hline & MAP1LC3B & $M$ & 74 & -0.34 & 0.11 \\
\hline & BECN1 & $M$ & 76 & -0.33 & 0.11 \\
\hline & BNIP3 & $M$ & 91 & -0.32 & 0.10 \\
\hline & ULK1 & $M$ & 73 & -0.30 & 0.09 \\
\hline & GABARAPL1 & $M$ & 94 & -0.29 & 0.08 \\
\hline & OPTN & $M$ & 96 & -0.27 & 0.07 \\
\hline & CALCOCO2 & M & 97 & -0.23 & 0.05 \\
\hline & BNIP3L & $M$ & 90 & -0.23 & 0.05 \\
\hline & ULK1 & $P$ & 30 & -0.20 & 0.04 \\
\hline & ULK1 ser555 & PP & 31 & -0.17 & 0.03 \\
\hline & MAP1LC3BI & $P$ & 35 & -0.15 & 0.02 \\
\hline & ULK1 ser757 & $\mathrm{PP}$ & 32 & -0.15 & 0.02 \\
\hline & MAP1LC3BII/I & $P$ & 37 & 0.14 & 0.02 \\
\hline & BNIP3LII & $P$ & 41 & -0.11 & 0.01 \\
\hline & SQSTM1 & $P$ & 38 & -0.06 & 0.00 \\
\hline & BNIP3L & $P$ & 40 & -0.06 & 0.00 \\
\hline & MAP1LC3BII & $P$ & 36 & 0.02 & 0.00 \\
\hline & BECN1 & $\mathrm{P}$ & 39 & 0.01 & 0.00 \\
\hline \multirow[t]{29}{*}{$\mathbf{P 2}$} & RPS6 ser235/236 & PP & 19 & -0.32 & 0.10 \\
\hline & AKT1 ser473 & PP & 13 & -0.32 & 0.10 \\
\hline & EIF4EBP1 ser65 & $\mathrm{PPr}$ & 23 & -0.28 & 0.08 \\
\hline & EIF4EBP1 ser65 & PP & 22 & -0.28 & 0.08 \\
\hline & MTOR ser2448 & PP & 16 & -0.27 & 0.07 \\
\hline & MTOR ser2448 & PPr & 17 & -0.27 & 0.07 \\
\hline & AKT1 ser473 & PPr & 14 & -0.26 & 0.07 \\
\hline & TBC1D1 thr642 & PP & 46 & -0.26 & 0.07 \\
\hline & RPS6 ser235/236 & $\mathrm{PPr}$ & 20 & -0.26 & 0.07 \\
\hline & TBC1D1 thr642 & PPr & 47 & -0.24 & 0.06 \\
\hline & FOXO1 thr24 & PPr & 29 & -0.20 & 0.04 \\
\hline & FOXO3 thr32 & PPr & 28 & -0.18 & 0.03 \\
\hline & ULK1 ser555 & $\mathrm{PPr}$ & 33 & -0.17 & 0.03 \\
\hline & TBC1D1 & $\mathrm{P}$ & 45 & -0.17 & 0.03 \\
\hline & GSK3B ser9 & $\mathrm{PPr}$ & 53 & -0.17 & 0.03 \\
\hline & ULK1 ser757 & $\mathrm{PPr}$ & 34 & -0.16 & 0.03 \\
\hline & RPS6 & $P$ & 18 & -0.12 & 0.01 \\
\hline & CCND1 & M & 66 & -0.12 & 0.01 \\
\hline & GSK3B & $P$ & 52 & 0.08 & 0.01 \\
\hline & PCNA & $M$ & 65 & -0.08 & 0.01 \\
\hline & GSK3B ser9 & $\mathrm{PP}$ & 51 & -0.07 & 0.00 \\
\hline & SLC2A1 & $M$ & 71 & 0.05 & 0.00 \\
\hline & DDIT4 & $M$ & 69 & 0.05 & 0.00 \\
\hline & SLC2A4 & $M$ & 72 & 0.05 & 0.00 \\
\hline & AKT1 & $P$ & 12 & -0.04 & 0.00 \\
\hline & EIF4EBP1 & $P$ & 21 & 0.03 & 0.00 \\
\hline & GYS1 ser641 & PP & 50 & -0.02 & 0.00 \\
\hline & MTOR & $P$ & 15 & -0.01 & 0.00 \\
\hline & PAX7 & $\mathrm{M}$ & 61 & 0.01 & 0.00 \\
\hline
\end{tabular}




\begin{tabular}{|c|c|c|c|c|c|}
\hline Process & Name & Type* & Number & $\begin{array}{l}\text { Component } \\
\text { loadings }\end{array}$ & $\begin{array}{l}\text { Squared component } \\
\text { loadings }\end{array}$ \\
\hline \multirow[t]{14}{*}{ P3 } & AKT1 ser308 & $\mathrm{PP}$ & 48 & -0.41 & 0.17 \\
\hline & IRS1 tyr632 & $\mathrm{PPr}$ & 11 & -0.39 & 0.15 \\
\hline & IRS1 ser612 & PPr & 9 & -0.39 & 0.15 \\
\hline & AKT1 ser308 & $\mathrm{PPr}$ & 49 & -0.38 & 0.14 \\
\hline & MYOD1 & $M$ & 58 & 0.31 & 0.10 \\
\hline & IRS1 ser612 & PP & 8 & -0.27 & 0.07 \\
\hline & IRS1 tyr632 & PP & 10 & -0.26 & 0.07 \\
\hline & $\mathrm{CDH} 15$ & $M$ & 60 & 0.26 & 0.07 \\
\hline & MYOG & $M$ & 64 & 0.21 & 0.04 \\
\hline & IRS1 & $P$ & 7 & -0.14 & 0.02 \\
\hline & IGF1 & $M$ & 62 & -0.11 & 0.01 \\
\hline & MYF5 & $M$ & 63 & 0.08 & 0.01 \\
\hline & MSTN & $M$ & 59 & 0.04 & 0.00 \\
\hline & MYMK & $\mathrm{M}$ & 101 & -0.01 & 0.00 \\
\hline \multirow[t]{6}{*}{ P4 } & UQCRC2 & $P$ & 2 & -0.46 & 0.21 \\
\hline & NDUFB8 & $\mathrm{P}$ & 0 & -0.44 & 0.19 \\
\hline & SDHB & $\mathrm{P}$ & 1 & -0.42 & 0.18 \\
\hline & ATP5F1A & $\mathrm{P}$ & 4 & -0.42 & 0.18 \\
\hline & MT-CO1 & $P$ & 3 & -0.38 & 0.15 \\
\hline & TOMM20 & $\mathrm{P}$ & 5 & -0.31 & 0.10 \\
\hline \multirow[t]{2}{*}{ P5 } & FUNDC1 & $P$ & 42 & -0.75 & 0.57 \\
\hline & DNM1L & $\mathrm{P}$ & 6 & -0.66 & 0.43 \\
\hline \multirow[t]{2}{*}{ P6 } & PRKNI & $P$ & 44 & 0.73 & 0.53 \\
\hline & PRKN & $\mathrm{P}$ & 43 & 0.69 & 0.47 \\
\hline \multirow[t]{13}{*}{ P7 } & KLF15 & $M$ & 68 & -0.35 & 0.12 \\
\hline & FOXO1 & $M$ & 54 & -0.34 & 0.12 \\
\hline & NFKBIA & $M$ & 67 & -0.33 & 0.11 \\
\hline & TRIM63 & $M$ & 56 & -0.32 & 0.10 \\
\hline & FOXO3 & $M$ & 55 & -0.31 & 0.10 \\
\hline & $\mathrm{FOXO} 3$ & $\mathrm{P}$ & 24 & -0.30 & 0.09 \\
\hline & FOXO1 & $\mathrm{P}$ & 25 & -0.28 & 0.08 \\
\hline & FOXO1 thr24 & PP & 27 & -0.26 & 0.07 \\
\hline & FBXO30 & $M$ & 100 & -0.25 & 0.06 \\
\hline & GLUL & $M$ & 70 & -0.23 & 0.05 \\
\hline & FBXO32 & $M$ & 57 & -0.22 & 0.05 \\
\hline & FOXO3 thr32 & $\mathrm{PP}$ & 26 & -0.19 & 0.04 \\
\hline & FBXO21 & $\mathrm{M}$ & 99 & -0.15 & 0.02 \\
\hline
\end{tabular}




\begin{tabular}{llllll}
\hline Process & Name & Type* & Number & $\begin{array}{l}\text { Component } \\
\text { loadings }\end{array}$ & $\begin{array}{l}\text { Squared component } \\
\text { loadings }\end{array}$ \\
\hline P8 & PINK1 & M & 93 & -0.31 & 0.09 \\
& OPA1 & M & 82 & -0.31 & 0.09 \\
& FIS1 & M & 81 & -0.30 & 0.09 \\
COX4I1 & M & 88 & -0.29 & 0.08 \\
MYH2 & M & 79 & -0.28 & 0.08 \\
MT-CO2 & M & 89 & -0.28 & 0.08 \\
MFN1 & M & 83 & -0.27 & 0.07 \\
TFAM & M & 87 & -0.27 & 0.07 \\
PPARGC1A & M & 84 & -0.24 & 0.06 \\
PPRC1 & M & 86 & -0.23 & 0.05 \\
PPARGC1B & M & 85 & -0.22 & 0.05 \\
PRKN & M & 95 & -0.21 & 0.05 \\
MYH7 & M & 77 & -0.20 & 0.04 \\
DNM1L & M & 80 & -0.20 & 0.04 \\
FUNDC1 & M & 92 & -0.17 & 0.03 \\
MYH1 & M & 78 & -0.13 & 0.02 \\
SOD2 & M & 98 & -0.07 & 0.00 \\
\hline
\end{tabular}

*Measurement level of indicated molecular markers; $M, m R N A$ expression; $P$, protein expression; $P P$ protein phosphorylation; $\mathrm{PPr}$, protein phosphorylation relative to total protein expression. Markers are ranked by strength of component loadings.

Table S3. | Rehabilitation responses per cluster, process scores

\begin{tabular}{llllll}
\hline Process & & Cluster $\mathbf{1}$ & Cluster 2 & $\mathbf{n}=$ & p-value* $^{*}$ \\
\hline P1 & Autophagy & $-0.38 \pm 0.07$ & $0.42 \pm 0.10$ & $29 / 16$ & $\mathbf{0 . 0 0 0}$ \\
P2 & AKT/mTOR signaling & $-0.06 \pm 0.09$ & $-0.06 \pm 0.08$ & $29 / 16$ & 0.989 \\
P3 & Myogenesis & $0.12 \pm 0.08$ & $-0.19 \pm 0.08$ & $29 / 16$ & $\mathbf{0 . 0 1 4}$ \\
P4 & OXCAP & $0.03 \pm 0.09$ & $-0.23 \pm 0.10$ & $29 / 16$ & 0.064 \\
P5 & Mitophagy, FUNDC1/DNM1L & $-0.02 \pm 0.06$ & $-0.02 \pm 0.10$ & $29 / 16$ & 0.993 \\
P6 & Mitophagy, PRKN & $0.04 \pm 0.08$ & $-0.09 \pm 0.09$ & $29 / 16$ & 0.275 \\
P7 & Glucocorticoid signaling & $-0.35 \pm 0.05$ & $0.49 \pm 0.14$ & $29 / 16$ & $\mathbf{0 . 0 0 0}$ \\
P8 & Oxidative metabolism regulation & $-0.39 \pm 0.06$ & $0.25 \pm 0.08$ & $29 / 16$ & $\mathbf{0 . 0 0 0}$ \\
\hline
\end{tabular}

*Independent sample t-test of rehabilitation responses in Cluster 1 vs. Cluster 2, with $p<0.05$ indicated in bold. Data expressed as mean \pm SEM. 


\section{CHAPTER 5}

Table S4. | Rehabilitation responses per cluster, molecular markers

\begin{tabular}{|c|c|c|c|c|c|c|c|}
\hline Process & Name & Typet & Number & Cluster 1 & Cluster 2 & $\mathrm{n}=$ & p-value $\ddagger$ \\
\hline \multirow[t]{19}{*}{ P1 } & SQSTM1 & $M$ & 75 & $-28.65 \pm 5.58 * * *$ & $26.69 \pm 8.85^{* *}$ & $28 / 16$ & 0.000 \\
\hline & MAP1LC3B & $M$ & 74 & $-23.06 \pm 2.53^{* * *}$ & $3.61 \pm 3.93$ & $28 / 16$ & 0.000 \\
\hline & BECN1 & $M$ & 76 & $-27.35 \pm 4.61 * * *$ & $2.35 \pm 6.27$ & $28 / 16$ & 0.000 \\
\hline & BNIP3 & $M$ & 91 & $-33.27 \pm 3.05^{* * *}$ & $-1.59 \pm 6.25$ & $28 / 16$ & 0.000 \\
\hline & ULK1 & $\mathrm{M}$ & 73 & $-23.85 \pm 4.83^{* * *}$ & $5.58 \pm 6.25$ & $28 / 15$ & 0.001 \\
\hline & GABARAPL1 & $M$ & 94 & $-28.36 \pm 6.18 * * *$ & $10.17 \pm 11.68$ & $28 / 15$ & 0.003 \\
\hline & OPTN & $M$ & 96 & $-44.84 \pm 3.18^{* * *}$ & $-19.78 \pm 6.85^{*}$ & $28 / 15$ & 0.003 \\
\hline & CALCOCO2 & $M$ & 97 & $-27.08 \pm 6.45^{* * *}$ & $92.19 \pm 75.07$ & $28 / 15$ & 0.135 \\
\hline & BNIP3L & $M$ & 90 & $1.70 \pm 13.50$ & $9.62 \pm 6.61$ & $28 / 16$ & 0.673 \\
\hline & ULK1 & $\mathrm{P}$ & 30 & $74.30 \pm 22.40^{* *}$ & $178.63 \pm 70.51^{*}$ & $27 / 13$ & 0.180 \\
\hline & ULK1 ser555 & PP & 31 & $27.84 \pm 10.30^{*}$ & $46.23 \pm 20.96^{*}$ & $27 / 13$ & 0.380 \\
\hline & MAP1LC3BI & $P$ & 35 & $13.02 \pm 7.57^{\#}$ & $45.74 \pm 12.65^{* *}$ & $27 / 13$ & 0.025 \\
\hline & ULK1 ser757 & PP & 32 & $30.92 \pm 7.10^{* * *}$ & $45.47 \pm 12.86^{* *}$ & $27 / 13$ & 0.289 \\
\hline & MAP1LC3BII/I & $\mathrm{P}$ & 37 & $46.20 \pm 12.86^{* *}$ & $5.21 \pm 12.30$ & $27 / 13$ & 0.052 \\
\hline & BNIP3LII & $\mathrm{P}$ & 41 & $111.09 \pm 30.73^{* *}$ & $92.86 \pm 27.33^{* *}$ & $27 / 13$ & 0.708 \\
\hline & SQSTM1 & $\mathrm{P}$ & 38 & $37.18 \pm 12.05^{* *}$ & $51.64 \pm 19.70^{*}$ & $27 / 13$ & 0.517 \\
\hline & BNIP3L & $\mathrm{P}$ & 40 & $7.00 \pm 4.13$ & $10.89 \pm 7.76$ & $27 / 13$ & 0.629 \\
\hline & MAP1LC3BII & $P$ & 36 & $55.69 \pm 16.13^{* *}$ & $41.37 \pm 12.43^{* *}$ & $27 / 13$ & 0.569 \\
\hline & BECN1 & $\mathrm{P}$ & 39 & $105.92 \pm 19.06^{* * *}$ & $140.14 \pm 44.61^{* *}$ & $27 / 13$ & 0.411 \\
\hline \multirow[t]{29}{*}{ P2 } & RPS6 ser235/236 & PP & 19 & $55.24 \pm 30.38^{\# \prime}$ & $64.72 \pm 34.74^{\#}$ & $29 / 16$ & 0.846 \\
\hline & AKT1 ser473 & PP & 13 & $14.75 \pm 9.22$ & $32.83 \pm 11.32 *$ & $29 / 16$ & 0.235 \\
\hline & EIF4EBP1 ser65 & $\mathrm{PPr}$ & 23 & $22.90 \pm 12.01^{\#}$ & $13.43 \pm 11.09$ & $29 / 16$ & 0.605 \\
\hline & EIF4EBP1 ser65 & $\mathrm{PP}$ & 22 & $12.66 \pm 10.74$ & $10.74 \pm 8.52$ & $29 / 16$ & 0.904 \\
\hline & MTOR ser2448 & PP & 16 & $22.31 \pm 8.42 *$ & $26.27 \pm 9.38^{*}$ & $29 / 16$ & 0.768 \\
\hline & MTOR ser2448 & $\mathrm{PPr}$ & 17 & $5.81 \pm 5.24$ & $9.13 \pm 5.35$ & $29 / 16$ & 0.684 \\
\hline & AKT1 ser473 & $\mathrm{PPr}$ & 14 & $-22.99 \pm 6.08^{* * *}$ & $-20.83 \pm 5.87^{* *}$ & $29 / 16$ & 0.817 \\
\hline & TBC1D1 thr642 & $\mathrm{PP}$ & 46 & $-15.47 \pm 5.38^{* *}$ & $-1.21 \pm 10.84$ & $29 / 16$ & 0.194 \\
\hline & RPS6 ser235/236 & $\mathrm{PPr}$ & 20 & $95.15 \pm 67.31$ & $21.90 \pm 29.42$ & $29 / 16$ & 0.438 \\
\hline & TBC1D1 thr642 & $\mathrm{PPr}$ & 47 & $-22.73 \pm 4.25 * * *$ & $-19.59 \pm 7.32 *$ & $29 / 16$ & 0.692 \\
\hline & FOXO1 thr24 & $\mathrm{PPr}$ & 29 & $3.97 \pm 4.10$ & $-6.71 \pm 4.23$ & $29 / 16$ & 0.100 \\
\hline & FOXO3 thr32 & PPr & 28 & $-2.95 \pm 5.25$ & $-16.05 \pm 4.59 * *$ & $29 / 16$ & 0.103 \\
\hline & ULK1 ser555 & $\mathrm{PPr}$ & 33 & $-11.68 \pm 8.35$ & $-23.58 \pm 16.64$ & $27 / 13$ & 0.479 \\
\hline & TBC1D1 & $\mathrm{P}$ & 45 & $10.78 \pm 4.65^{*}$ & $23.67 \pm 10.12 *$ & $29 / 16$ & 0.193 \\
\hline & GSK3B ser9 & $\mathrm{PPr}$ & 53 & $-7.19 \pm 2.20 * *$ & $-5.84 \pm 3.76$ & $29 / 16$ & 0.741 \\
\hline & ULK1 ser757 & $\mathrm{PPr}$ & 34 & $-3.40 \pm 8.74$ & $-26.59 \pm 8.71 *$ & $27 / 13$ & 0.106 \\
\hline & RPS6 & $\mathrm{P}$ & 18 & $19.28 \pm 13.24$ & $62.49 \pm 22.19 *$ & $29 / 16$ & 0.082 \\
\hline & CCND1 & $\mathrm{M}$ & 66 & $82.64 \pm 27.00^{* *}$ & $87.57 \pm 34.82^{*}$ & $28 / 15$ & 0.913 \\
\hline & GSK3B & $P$ & 52 & $15.29 \pm 3.61^{* * *}$ & $26.68 \pm 8.00^{* *}$ & $29 / 16$ & 0.208 \\
\hline & PCNA & M & 65 & $40.18 \pm 37.33$ & $50.97 \pm 47.33$ & $28 / 15$ & 0.862 \\
\hline & GSK3B ser9 & PP & 51 & $5.32 \pm 2.15^{*}$ & $16.89 \pm 6.32^{*}$ & $29 / 16$ & 0.099 \\
\hline & SLC2A1 & $M$ & 71 & $72.78 \pm 34.24 *$ & $60.73 \pm 30.09^{\#}$ & $28 / 16$ & 0.814 \\
\hline & DDIT4 & $M$ & 69 & $-26.65 \pm 9.26 * *$ & $81.19 \pm 32.00 *$ & $28 / 15$ & 0.005 \\
\hline & SLC2A4 & $\mathrm{M}$ & 72 & $-38.04 \pm 7.28 * * *$ & $18.14 \pm 7.13^{*}$ & $28 / 16$ & 0.000 \\
\hline & AKT1 & $\mathrm{P}$ & 12 & $53.58 \pm 7.78^{* * *}$ & $78.06 \pm 18.63^{* * *}$ & $29 / 16$ & 0.239 \\
\hline & EIF4EBP1 & $\mathrm{P}$ & 21 & $-7.27 \pm 2.09 * *$ & $2.54 \pm 6.08$ & $29 / 16$ & 0.144 \\
\hline & GYS1 ser641 & PP & 50 & $158.72 \pm 43.29 * *$ & $249.85 \pm 190.68$ & $29 / 16$ & 0.552 \\
\hline & MTOR & $\mathrm{P}$ & 15 & $15.29 \pm 3.70^{* * *}$ & $16.65 \pm 8.00^{\#}$ & $29 / 16$ & 0.879 \\
\hline & PAX7 & $M$ & 61 & $-7.53 \pm 11.72$ & $37.27 \pm 21.98$ & $28 / 15$ & 0.055 \\
\hline
\end{tabular}




\begin{tabular}{|c|c|c|c|c|c|c|c|}
\hline Process & Name & Typet & Number & Cluster 1 & Cluster 2 & $n=$ & p-value $\ddagger$ \\
\hline \multirow[t]{14}{*}{ P3 } & AKT1 ser308 & $\mathrm{PP}$ & 48 & $101.20 \pm 35.16^{* *}$ & $42.03 \pm 29.47$ & $29 / 16$ & 0.264 \\
\hline & IRS1 tyr632 & $\mathrm{PPr}$ & 11 & $13.79 \pm 11.85$ & $-11.15 \pm 8.07$ & $29 / 16$ & 0.152 \\
\hline & IRS1 ser612 & PPr & 9 & $-48.37 \pm 5.06 * * *$ & $-59.31 \pm 6.76 * * *$ & $29 / 16$ & 0.203 \\
\hline & AKT1 ser308 & $\mathrm{PPr}$ & 49 & $49.22 \pm 33.52$ & $-0.07 \pm 27.20$ & $29 / 16$ & 0.326 \\
\hline & MYOD1 & $\mathrm{M}$ & 58 & $-33.00 \pm 8.05 * * *$ & $20.54 \pm 18.59$ & $28 / 16$ & 0.015 \\
\hline & IRS1 ser612 & PP & 8 & $5.16 \pm 10.81$ & $-7.53 \pm 9.77$ & $29 / 16$ & 0.440 \\
\hline & IRS1 tyr632 & PP & 10 & $151.12 \pm 33.32 * * *$ & $129.08 \pm 24.54 * * *$ & $29 / 16$ & 0.652 \\
\hline & $\mathrm{CDH} 15$ & M & 60 & $-33.13 \pm 5.00 * * *$ & $26.56 \pm 13.58^{\#}$ & $28 / 15$ & 0.000 \\
\hline & MYOG & M & 64 & $13.45 \pm 8.92$ & $43.95 \pm 12.67^{* *}$ & $28 / 16$ & 0.051 \\
\hline & IRS1 & $P$ & 7 & $1.78 \pm 7.90$ & $6.18 \pm 9.19$ & $29 / 16$ & 0.729 \\
\hline & IGF1 & $M$ & 62 & $56.71 \pm 18.30^{* *}$ & $70.85 \pm 26.31 *$ & $28 / 16$ & 0.654 \\
\hline & MYF5 & $M$ & 63 & $24.08 \pm 25.88$ & $53.34 \pm 26.43^{\#}$ & $28 / 15$ & 0.473 \\
\hline & MSTN & $M$ & 59 & $18.30 \pm 20.50$ & $33.91 \pm 15.19^{*}$ & $28 / 16$ & 0.600 \\
\hline & MYMK & $\mathrm{M}$ & 101 & $569.35 \pm 185.41^{* *}$ & $370.03 \pm 172.75^{\#}$ & $25 / 12$ & 0.503 \\
\hline \multirow[t]{6}{*}{ P4 } & UQCRC2 & $P$ & 2 & $2.98 \pm 4.73$ & $-8.35 \pm 8.01$ & $29 / 16$ & 0.200 \\
\hline & NDUFB8 & $P$ & 0 & $52.71 \pm 22.94 *$ & $2.93 \pm 17.79$ & $29 / 16$ & 0.147 \\
\hline & SDHB & $P$ & 1 & $13.17 \pm 4.30^{* *}$ & $1.77 \pm 6.83$ & $29 / 16$ & 0.145 \\
\hline & ATP5F1A & $P$ & 4 & $9.78 \pm 2.74 * *$ & $-2.09 \pm 8.43$ & $29 / 16$ & 0.108 \\
\hline & MT-CO1 & $P$ & 3 & $53.83 \pm 24.17^{*}$ & $-6.93 \pm 19.29$ & $29 / 16$ & 0.096 \\
\hline & TOMM20 & $\mathrm{P}$ & 5 & $31.43 \pm 8.21^{* * *}$ & $16.63 \pm 11.49$ & $27 / 13$ & 0.306 \\
\hline \multirow[t]{2}{*}{ P5 } & FUNDC1 & $P$ & 42 & $13.22 \pm 4.19 * *$ & $14.02 \pm 8.40$ & $27 / 13$ & 0.925 \\
\hline & DNM1L & $\mathrm{P}$ & 6 & $29.26 \pm 5.73^{* * *}$ & $27.73 \pm 8.61^{* *}$ & $27 / 13$ & 0.881 \\
\hline \multirow[t]{2}{*}{ P6 } & PRKNI & $P$ & 44 & $-35.08 \pm 6.59 * * *$ & $-26.06 \pm 8.59 *$ & $27 / 13$ & 0.427 \\
\hline & PRKN & $\mathrm{P}$ & 43 & $-24.67 \pm 4.43^{* * *}$ & $-17.84 \pm 5.56^{* *}$ & $27 / 13$ & 0.366 \\
\hline \multirow[t]{13}{*}{ P7 } & KLF15 & $\mathrm{M}$ & 68 & $-57.68 \pm 4.06 * * *$ & $10.08 \pm 20.68$ & $28 / 16$ & 0.005 \\
\hline & FOXO1 & $M$ & 54 & $-36.90 \pm 8.58 * * *$ & $28.04 \pm 21.96$ & $28 / 16$ & 0.012 \\
\hline & NFKBIA & $M$ & 67 & $-24.20 \pm 10.52^{*}$ & $124.09 \pm 43.13^{*}$ & $28 / 16$ & 0.004 \\
\hline & TRIM63 & $M$ & 56 & $-41.41 \pm 5.30 * * *$ & $10.34 \pm 13.86$ & $28 / 16$ & 0.002 \\
\hline & FOXO3 & $M$ & 55 & $-27.34 \pm 16.57$ & $101.05 \pm 63.03$ & $28 / 16$ & 0.065 \\
\hline & FOXO3 & $P$ & 24 & $-3.16 \pm 4.55$ & $35.47 \pm 9.61 * *$ & $29 / 16$ & 0.001 \\
\hline & FOXO1 & $\mathrm{P}$ & 25 & $-11.54 \pm 7.04$ & $45.39 \pm 17.47^{*}$ & $29 / 16$ & 0.001 \\
\hline & FOXO1 thr24 & PP & 27 & $-9.11 \pm 7.67$ & $33.21 \pm 15.85^{\#}$ & $29 / 16$ & 0.010 \\
\hline & FBXO30 & M & 100 & $-19.97 \pm 6.38^{* *}$ & $8.93 \pm 9.79$ & $28 / 15$ & 0.014 \\
\hline & GLUL & $M$ & 70 & $-48.71 \pm 4.53^{* * *}$ & $6.37 \pm 11.45$ & $28 / 16$ & 0.000 \\
\hline & $\mathrm{FBXO32}$ & $M$ & 57 & $-39.47 \pm 6.54 * * *$ & $-16.84 \pm 8.66^{\#}$ & $28 / 16$ & 0.043 \\
\hline & FOXO3 thr32 & PP & 26 & $-8.01 \pm 5.51$ & $11.68 \pm 7.63$ & $29 / 16$ & 0.041 \\
\hline & $\mathrm{FBXO} 21$ & $\mathrm{M}$ & 99 & $-30.26 \pm 5.34 * * *$ & $1.08 \pm 8.86$ & $28 / 15$ & 0.003 \\
\hline \multirow[t]{17}{*}{ P8 } & PINK1 & $\mathrm{M}$ & 93 & $0.19 \pm 7.41$ & $51.29 \pm 12.22^{* * *}$ & $28 / 16$ & 0.000 \\
\hline & OPA1 & M & 82 & $-15.40 \pm 4.17^{* * *}$ & $6.49 \pm 6.46$ & $28 / 16$ & 0.005 \\
\hline & FIS1 & $M$ & 81 & $-29.07 \pm 2.36 * * *$ & $-0.17 \pm 4.68$ & $28 / 16$ & 0.000 \\
\hline & coX4l1 & $M$ & 88 & $-14.21 \pm 4.53^{* *}$ & $5.18 \pm 3.59$ & $28 / 16$ & 0.005 \\
\hline & MYH2 & $M$ & 79 & $-19.03 \pm 6.45^{* *}$ & $12.60 \pm 6.27^{\#}$ & $28 / 16$ & 0.002 \\
\hline & MT-CO2 & $M$ & 89 & $-10.45 \pm 4.88^{*}$ & $3.76 \pm 6.31$ & $28 / 15$ & 0.088 \\
\hline & MFN1 & $M$ & 83 & $-15.39 \pm 2.91 * * *$ & $6.13 \pm 5.29$ & $28 / 16$ & 0.000 \\
\hline & TFAM & M & 87 & $-28.82 \pm 3.54 * * *$ & $-12.20 \pm 3.80 * *$ & $28 / 16$ & 0.004 \\
\hline & PPARGC1A & M & 84 & $-36.50 \pm 6.70 * * *$ & $10.86 \pm 21.45$ & $28 / 16$ & 0.049 \\
\hline & PPRC1 & M & 86 & $-26.72 \pm 3.13 * * *$ & $-7.01 \pm 4.96$ & $28 / 16$ & 0.001 \\
\hline & PPARGC1B & $M$ & 85 & $-25.02 \pm 6.61 * * *$ & $8.52 \pm 14.32$ & $28 / 16$ & 0.045 \\
\hline & PRKN & $M$ & 95 & $-25.91 \pm 9.91 *$ & $53.77 \pm 31.12$ & $27 / 15$ & 0.026 \\
\hline & MYH7 & $M$ & 77 & $-25.77 \pm 14.15^{\#}$ & $111.29 \pm 96.54$ & $28 / 16$ & 0.180 \\
\hline & DNM1L & M & 80 & $3.28 \pm 21.38$ & $7.95 \pm 7.97$ & $28 / 16$ & 0.873 \\
\hline & FUNDC1 & $M$ & 92 & $-20.64 \pm 5.77 * *$ & $-9.34 \pm 6.54$ & $28 / 15$ & 0.228 \\
\hline & MYH1 & $M$ & 78 & $-27.43 \pm 9.13^{* *}$ & $54.64 \pm 35.90$ & $28 / 16$ & 0.041 \\
\hline & SOD2 & $M$ & 98 & $-18.50 \pm 5.27 * *$ & $17.35 \pm 9.12^{\#}$ & $28 / 15$ & 0.001 \\
\hline
\end{tabular}

$M, m R N A$ expression; $P$, protein expression; PP protein phosphorylation; $P P r$, protein phosphorylation relative to total protein expression. Data expressed as mean \pm SEM. ${ }^{*} p<0.05,{ }^{* *} p<0.01,{ }^{* * *} p<0.001$, comparing pre-and post-rehabilitation by paired-sample t-test. + Measurement level of indicated 


\section{CHAPTER 5}

molecular markers. ¥Independent sample t-test of rehabilitation responses in Cluster 1 vs. Cluster 2, with $p<0.05$ indicated in bold.

\begin{tabular}{|c|c|c|c|}
\hline & Cluster 1 & Cluster 2 & p-value* \\
\hline Inhaled corticosteroids, n (\%) & $19(76)$ & $10(77)$ & ns \\
\hline Short-acting beta-agonists, n (\%) & $22(88)$ & $11(85)$ & ns \\
\hline Long-acting beta-agonists, n (\%) & $24(96)$ & $10(77)$ & $<0.1$ \\
\hline Long-acting muscarinic antagonists, $\mathrm{n}(\%)$ & $22(88)$ & $10(77)$ & ns \\
\hline Theophylline, n (\%) & $7(28)$ & $1(8)$ & ns \\
\hline ACE-inhibitors, n (\%) & $8(32)$ & $4(31)$ & ns \\
\hline Angiotensin II receptor blockers, $\mathrm{n}(\%)$ & $1(4)$ & $1(8)$ & ns \\
\hline Calcium-channel blockers, $\mathrm{n}(\%)$ & $2(8)$ & $2(15)$ & ns \\
\hline Loop diuretics, n (\%) & $2(8)$ & $1(8)$ & ns \\
\hline Beta-blockers, n (\%) & $3(12)$ & $0(0)$ & ns \\
\hline Proton-pump inhibitor, $\mathrm{n}(\%)$ & $7(29)$ & $1(8)$ & ns \\
\hline Statins, n (\%) & $6(24)$ & $2(15)$ & ns \\
\hline Metformin, n (\%) & $1(4)$ & $0(0)$ & ns \\
\hline Insulin, n (\%) & $0(0)$ & $1(8)$ & ns \\
\hline Vitamin D, n (\%) & $3(12)$ & $0(0)$ & ns \\
\hline Calciumcarbonate, n (\%) & $4(16)$ & $2(15)$ & ns \\
\hline Other diuretics, n (\%) & $3(13)$ & $1(8)$ & ns \\
\hline
\end{tabular}

${ }^{*}$ Chi-square test of Cluster 1 vs. Cluster 2. 


\section{A NOVEL IN VITRO MODEL FOR X THE}

ASSESSMENT OF POSTNATAL MYONUCLEAR ACCRETION

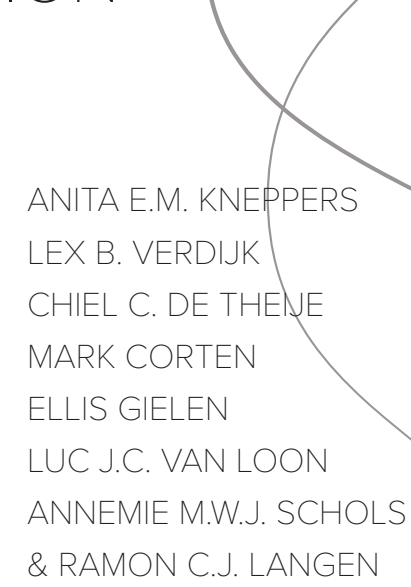

Skelet Muscle 2018;8(1):4. 


\section{CHAPTER 6}

\section{ABSTRACT}

Background: Due to the post-mitotic nature of myonuclei, postnatal myogenesis is essential for skeletal muscle growth, repair, and regeneration. This process is facilitated by satellite cells through proliferation, differentiation, and subsequent fusion with a pre-existing muscle fiber (i.e., myonuclear accretion). Current knowledge of myogenesis is primarily based on the in vitro formation of syncytia from myoblasts, which represents aspects of developmental myogenesis, but may incompletely portray postnatal myogenesis. Therefore, we aimed to develop an in vitro model that better reflects postnatal myogenesis, to study the cell intrinsic and extrinsic processes and signaling involved in the regulation of postnatal myogenesis.

Methods: Proliferating C2C12 myoblasts were trypsinized and co-cultured for 3 days with 5 days differentiated $\mathrm{C} 2 \mathrm{C} 12$ myotubes. Postnatal myonuclear accretion was visually assessed by live cell time-lapse imaging and cell tracing by cell labelling with Vybrant ${ }^{\circledast}$ DiD and DiO. Furthermore, a Cre/LoxP-based cell system was developed to semi-quantitatively assess in vitro postnatal myonuclear accretion by the conditional expression of luciferase upon myoblast-myotube fusion. Luciferase activity was assessed luminometrically and corrected for total protein content.

Results: Live cell time-lapse imaging, staining-based cell tracing, and recombinationdependent luciferase activity, showed the occurrence of postnatal myonuclear accretion in vitro. Treatment of co-cultures with the myogenic factor IGF-I $(p<0.001)$, and the cytokines IL-13 $(p<0.05)$ and IL-4 $(p<0.001)$ increased postnatal myonuclear accretion, while the myogenic inhibitors Cytochalasin D $(p<0.001)$, Myostatin $(p<0.05)$, and TNFa $(p<0.001)$ decreased postnatal myonuclear accretion. Furthermore, postnatal myonuclear accretion was increased upon recovery from electrical pulse stimulationinduced fiber damage $(p<0.001)$ and LY29004-induced atrophy $(p<0.001)$. Moreover, cell type-specific siRNA-mediated knockdown of Myomaker in myoblasts $(p<0.001)$, but not in myotubes, decreased postnatal myonuclear accretion.

Conclusions: We developed a physiologically relevant, sensitive, high-throughput cell system for semi-quantitative assessment of in vitro postnatal myonuclear accretion, which can be used to mimic physiological myogenesis triggers, and can distinguish the cell type-specific roles of signals and responses in the regulation of postnatal myogenesis. As such, this method is suitable for both basal and translational research on the regulation of postnatal myogenesis, and will improve our understanding of muscle pathologies that result from impaired satellite cell number or function. 
Acknowledgements

The authors wish to thank Arjan Groot (MAASTRO, Maastricht University) for providing technical assistance with live cell imaging, and Leonor Keating and Cristina Nardone for their assistance in the performance of experiments. Furthermore, the authors thank Vincent Mouly (Pierre and Marie Curie University, Institute of Myology) for providing the Human C25 myoblasts. Cre-IRES-PuroR was a gift from Darrell Kotton, pRSV-Rev, pMDLg/pRRE and pMD2.G were a gift from Didier Trono, and LV-floxed-Luc was a gift from Charles Gersbach.

\section{BACKGROUND}

Skeletal muscle fibers arise from the biochemical and morphological differentiation of muscle precursor cells. This process is called myogenesis and is essential for prenatal development, as well as postnatal muscle tissue growth, maintenance, and repair.

Myogenesis during prenatal skeletal muscle development is elaborately studied (for review, see e.g. Buckingham et al. [1]). Briefly, limb muscles arise from muscle progenitor cells that are derived from the dermomyotome - the maturing somite that forms from the paraxial mesoderm. Upon external cues, muscle progenitor cells migrate into the limb bud, proliferate, become committed to the myogenic transcription program, differentiate biochemically and morphologically, and fuse with each other to give rise to primary and secondary muscle fibers.

In postnatal skeletal muscle, muscle progenitor cells are also present. These adult progenitor cells, called satellite cells, originate from the same dermomyotomederived $\mathrm{Pax}^{+} / \mathrm{Pax}^{+}$population [2-5] and are located between the sarcolemma and basal lamina. In the early postnatal period, myogenesis facilitates muscle growth by satellite cell proliferation, differentiation, and fusion with a pre-existing muscle fiber [6], which is recapitulated during adult muscle hypertrophy. However, in healthy adult muscle, the majority of satellite cells are quiescent. Upon external cues, adult satellite cells become activated, proliferate, and either return to quiescence in a process called self-renewal, or differentiate to fuse with a pre-existing muscle fiber (for review, see e.g. Dhawan et al. [7]).

Due to the post-mitotic nature of myonuclei, postnatal myogenesis is essential for skeletal muscle maintenance, growth, repair, and regeneration. In vivo in human skeletal muscle, these roles are demonstrated by the acute increase in satellite cell activation status and number after exercise, and the positive association between changes in muscle fiber size, satellite cell content, and myonuclear content after prolonged exercise training, as reviewed by Snijders et al. [8]. The requirement of satellite cells for muscle growth, repair, and regeneration has mainly been established in animal studies. These fundamental studies showed that satellite cell ablation by $\gamma$-irradiation or $\mathrm{Pax}^{+}$cell depletion prevents sustained overload-induced muscle 


\section{CHAPTER 6}

hypertrophy and recovery from muscle injury [9-12]. Although subsequent work has challenged this concept [13], more recent studies confirm the essential role of satellite cells in muscle growth $[14,15]$.

A role for impaired myogenesis, by either a reduced satellite cell number or function, has been implicated in the loss of skeletal muscle mass and function (i.e., sarcopenia) in aging, chronic diseases, and myopathies [16-18]. The identification of regulators and pathways involved in postnatal satellite cell function and dysfunction is imperative for a better comprehension of the role of myogenesis in aging and disease-related muscle loss, and to develop targeted intervention strategies aimed at optimizing muscle health and function.

Considerable progress has been made towards uncovering these mediators and regulators of satellite cell function in health and disease, as reviewed by Buckingham et al. [1]. In vivo and ex vivo models were fundamental in their identification, while knowledge on the causal role of these mediators and regulators in myogenesis was primarily based on in vitro culturing and differentiation of primary satellite cells and immortalized myoblasts. This in vitro formation of syncytia from mononucleated myoblasts morphologically resembles developmental myogenesis, but has also been employed as a model to study the regulation of postnatal myogenesis [1921]. Indeed, it was theorized that muscle regeneration recapitulates developmental myogenesis. However, based on the reported differential requirements for $\beta$-catenin during embryonic and fetal myogenesis [5], this theory has been challenged [2224]. Several reviews compared developmental and postnatal myogenesis and found important distinctions in the involved cells, anatomy, cellular mechanisms, and expression of regulatory genes [22, 24, 25]. This novel insight requires reframing of the current knowledge on myogenesis, and reconsideration of established myogenesis models. Specifically, we should consider the contextual use of the classical in vitro model of myogenesis, i.e., syncytia formation from myoblasts, which may represent developmental myogenesis, but incompletely or even inaccurately portrays postnatal myogenesis.

The use of a more appropriate model will advance the comprehension of regulation and dysregulation of postnatal myogenesis and its role in the development of sarcopenia, and is thus indispensable in the identification of specific targets for effective prevention and treatment of sarcopenia. In this study, we therefore aimed to develop an in vitro model that better reflects postnatal myogenesis, to study the cell intrinsic and extrinsic processes and signaling involved in the regulation of postnatal myogenesis. 


\section{METHODS}

\section{Cell culture and reagents}

Cell maintenance and experiments were performed in a humidified incubator at $37^{\circ} \mathrm{C}$ with $5 \% \mathrm{CO}_{2}$. C2C12 myoblasts (ATCC, Wesel, Germany; \#CRL-1772) were maintained in growth medium (GM) which was composed of Dulbecco's modified Eagle's medium (DMEM $1 \mathrm{~g} / \mathrm{L}$ glucose (Gibco, Rockville, MD; \#22320-022) supplemented with 9\% (v/v) fetal bovine serum (FBS), and $50 \mathrm{U} / \mathrm{mL}$ penicillin and $50 \mu \mathrm{g} / \mathrm{mL}$ streptomycin (P/S) (Gibco). To induce differentiation, cells were plated onto tissue culture plates coated with $2 \%(\mathrm{v} / \mathrm{v})$ Growth Factor Reduced Matrigel ${ }^{\circledR}$ (BD Biosciences, Bedford, MA) and switched to differentiation medium (DM) composed of DMEM $4.5 \mathrm{~g} / \mathrm{L}$ glucose (Gibco; \#41966-029) supplemented with $0.5 \%$ (v/v) heat-inactivated FBS and P/S.

Human C25 myoblasts (HSM) [26] were kindly provided by V. Mouly. HSM cells were maintained in GM which was composed of Skeletal Muscle Cell Growth Medium supplemented with $42 \mu \mathrm{g} / \mathrm{mL}$ bovine Fetuin, $8.3 \mathrm{ng} / \mathrm{mL}$ human recombinant Epidermal Growth Factor, $0.83 \mathrm{ng} / \mathrm{mL}$ human recombinant Basic Fibroblast Growth Factor, 8.3 $\mu \mathrm{g} / \mathrm{mL}$ human recombinant Insulin, $0.33 \mu \mathrm{g} / \mathrm{mL}$ Dexamethasone (all from PromoCell, Heidelberg, Germany), 17\% (v/v) FBS, and P/S. To induce differentiation, cells were plated onto Matrigel-coated tissue culture plates and switched to DM which was composed of DMEM 4.5 g/L glucose, GlutaMAX ${ }^{\mathrm{TM}}$ (Gibco; \#61965-026) supplemented with $\mathrm{P} / \mathrm{S}$.

DM was replaced $24 \mathrm{~h}$ after initiation of differentiation, and subsequently every 48 h. Myotube-myoblast co-cultures were initiated with 5 days differentiated myotubes and 5000 cells $/ \mathrm{cm}^{2}$ myoblasts, unless indicated otherwise.

For lentivirus production, 293FT cells (Invitrogen, Carlsbad, CA; \#R700-07) were maintained in DMEM $4.5 \mathrm{~g} / \mathrm{L}$ glucose supplemented with $9 \%(\mathrm{v} / \mathrm{v}) \mathrm{FBS}, \mathrm{P} / \mathrm{S}$, and 500 $\mu \mathrm{g} / \mathrm{mL}$ Geneticin (Gibco).

Recombinant human insulin-like growth factor 1 (IGF-I) (Sigma-Aldrich, Zwijndrecht, the Netherlands), recombinant human/mouse/rat growth differentiation factor 8 (Myostatin, MSTN), recombinant mouse interleukin-4 (IL-4) and human IL-13 (R\&D systems, Minneapolis, MN), and recombinant mouse tumor necrosis factor alpha (TNF-a) (Merck Millipore, Amsterdam, the Netherlands) were dissolved in $0.1 \%$ bovine serum albumin (BSA) (Sigma-Aldrich) in Hanks'Balanced Salt Solution (HBSS). LY294002 (LY) (Merck Millipore) and Cytochalasin D (CytoD) (Sigma-Aldrich) were dissolved in DMSO. 


\section{CHAPTER 6}

\section{Electrical pulse stimulation}

Myotubes were electrically stimulated using a C-Pace unit and a C-Dish electrode assembly for $35 \mathrm{~mm}$ culture dishes (Ion Optix, Milton, MA). Stimulation was performed according to the "twitch" protocol by Orfanos et al. [27]. Briefly, pulses were applied for $20 \mathrm{~ms}$ at $10 \mathrm{~V}$, at a frequency of $1 \mathrm{~Hz}$.

\section{RNA interference}

Knockdown of Myomaker was achieved by RNA interference. Target Silencer ${ }^{\circledR}$ Select siRNA or negative control Silencer ${ }^{\circledR}$ Select siRNA (final $10 \mathrm{nM}$ ) was mixed with Lipofectamine RNAiMAX (Invitrogen) in Opti-MEM reduced serum medium (Gibco; \#31985-070). After complex formation at room temperature (RT) for $5 \mathrm{~min}$, the transfection mix was added to adherent cells, and incubated for $24 \mathrm{~h}$ before initiation of co-culturing.

\section{Western blot}

Cells were lysed in whole cell lysis buffer (20 mM Tris, $150 \mathrm{mM} \mathrm{NaCl}, 1 \%$ Nonidet P40, and protease and phosphatase inhibitors (Roche)), incubated on ice for $30 \mathrm{~min}$, and centrifuged at $14,000 \mathrm{~g}$ for $30 \mathrm{~min}$. Total protein concentration in the supernatant was determined using BCA Protein Assay kit (Pierce) according to the manufacturer's instructions. 4x Laemmli sample buffer (0.25 M Tris-HCl ph 6.8, 8\% (w/v) SDS, 40\% (v/v) glycerol, $0.4 \mathrm{M} \mathrm{DTT}$, and $0.02 \%$ (w/v) Bromophenol Blue) was added to the supernatant, and samples were heated to $100^{\circ} \mathrm{C}$ for $5 \mathrm{~min}$. Protein $(3 \mu \mathrm{g}$ myoblast lysate; $10 \mu \mathrm{g}$ myotube lysate) were separated on a Criterion XT Precast 4-12\% Bis-Tris gel (Bio-Rad), followed by transfer to a $0.45 \mu \mathrm{m}$ nitrocellulose membrane (Bio-Rad) by electroblotting. For total protein detection, the membrane was stained with PonceauS solution $(0.2 \%$ PonceauS in 1\% acetic acid; Sigma-Aldrich Chemie) and imaged using the Amersham imager 600RGB. The membrane was blocked for $1 \mathrm{~h}$ at RT in Tris-buffered saline with Tween20 (20 mM Tris, 137 mM NaCl, 0.1\% (v/v) Tween20, pH 7.6 (TBST)) containing 5\% $(\mathrm{w} / \mathrm{v})$ nonfat dry milk (Campina). After washing in TBST, the membrane was incubated overnight at $4^{\circ} \mathrm{C}$ with 1:1000 anti-Myomaker (NBP2-34175; Novus) diluted in TBST with $5 \%(\mathrm{w} / \mathrm{v}) \mathrm{BSA}$. Subsequently, the membrane was incubated with peroxidase conjugated secondary antibody solution (PI-1000; Vector laboratories) for $1 \mathrm{~h}$ at RT, and the target was visualized by chemiluminescence using Supersignal FEMTO Chemiluminescent substrate (Pierce Biotechnology, Inc.) according to the manufacturer's instructions, and detected using the Amersham Imager 600RGB. Signals were quantified with Image Quant Software (Amersham), and corrected for total protein content. 


\section{Stable transfection}

The LV-floxed-Luc C2C12 cell line expresses a conditional luciferase cassette dependent on Cre-mediated recombination, and was generated by Lipofectamine ${ }^{\circledR}$ 2000 (Invitrogen) mediated transfection with LV-floxed-Luc (Addgene, Cambridge, MA; \#60622) and subsequent selection based on a selectable marker.

The Cre C2C12 cell line expresses Cre recombinase, and was obtained by lentivirusmediated infection with Cre-IRES-PuroR (Addgene; \#30205). Lentiviral supernatant was produced by polyethylenimine-mediated co-transfection of 293FT cells with Cre-IRESPuroR, pRSV-Rev (Addgene; \#12253), pMDLg/pRRE (Addgene; \#12251), and pMD2.G (Addgene; \#12259) (1:1:1:1) in C2C12 GM. Lentivirus supernatant was collected 24, 48, and $72 \mathrm{~h}$ after transfection, filter sterilized $(0.45 \mu \mathrm{M})$, and stored at $-20^{\circ} \mathrm{C}$. C2C12 cells were infected by addition of lentiviral supernatant in GM (1:1) containing $1 \mu \mathrm{g} / \mathrm{mL}$ polybrene (Sigma-Aldrich), and subsequently selected based on a selectable marker.

Stable polyclonal cell lines were expanded, and stored in liquid nitrogen. For each experiment, a new vial was used to reduce variability between experiments.

\section{Cell staining and hybrid quantification}

$\mathrm{C} 2 \mathrm{C} 12$ or HSM cells were subjected to live staining with Vybrant ${ }^{\circledast} \mathrm{DiD}$ or DiO cell labelling solution (Life Technologies, Carlsbad, CA) before plating. Briefly, myoblasts were stained in suspension in $\mathrm{GM}$ at $37^{\circ} \mathrm{C}$ for $20 \mathrm{~min}$, and washed three times by pelleting and resuspension in GM. To obtain stained myotubes, stained myoblasts were plated and differentiated as described. Subsequently, co-culturing was initiated, and after 2 days, cell monolayers were washed with HBSS and fixed in 3.7\% paraformaldehyde (PFA). After fixation, nuclei were stained with DAPI and coverslips were mounted onto the cell monolayers using Mowiol/DABCO.

Images were taken at a 100x magnification using an Eclipse E800 microscope (Nikon) connected to a digital camera (DXM, 1200 NF, Nikon). For quantification of hybrid formation, five random fields of view (FOV) were captured from each well. Myotubes (i.e., cells with $\geq 3$ nuclei) containing both $\mathrm{DiD}$ and DiO cell tracers, resulting from myonuclear accretion, were defined as hybrids. Total myotubes and hybrid myotubes were counted, and hybrids were expressed as percentage of the total number of myotubes. The sum of five images was used to represent each well. 


\section{CHAPTER 6}

\section{BrdU labelling and immunohistochemical detection}

C2C12 myoblasts were incubated with $10 \mu \mathrm{M}$ Bromodeoxyuridine (BrdU) (SigmaAldrich) in GM for 24 h, and subsequently co-cultured with 5 days differentiated myotubes.

At the indicated time points, cells were fixed in 3.7\% PFA, and subsequently permeabilized with $0.1 \%$ Triton X-100 (Sigma-Aldrich) in phosphate buffered saline (PBS). Fixed cells were denatured with $1 \mathrm{~N} \mathrm{HCl}$ in PBS for 30 min at $45^{\circ} \mathrm{C}$, and neutralized with $0.1 \mathrm{M}$ borate buffer $(\mathrm{pH}$ 8.5) for $10 \mathrm{~min}$ at RT. After $1 \mathrm{~h}$ incubation with blocking buffer (1\% BSA, $22.52 \mathrm{mg} / \mathrm{ml}$ glycine, 0.1\% Tween in PBS (PBST)), cells were incubated overnight with rat monoclonal anti-BrdU antibody (Abcam; \#ab6326) (1:75 in 1\% BSA in PBS) at $4^{\circ} \mathrm{C}$ in a humidified chamber, and then incubated for $1 \mathrm{~h}$ with rabbit anti-rat antibody (Abcam; \#ab6730) (1:200 in 1\% BSA in PBS) at RT in the dark. Nuclei were subsequently stained with DAPI, and coverslips were mounted onto the cell monolayers using Mowiol/DABCO. Images were taken at a 100x magnification using an Axio Observer A1 microscope (Zeiss) connected to a digital camera (AxioCam ICM1, Zeiss). From each well, five random FOV were captured, and $\mathrm{BrdU}^{+}$nuclei (staining positive for BrdU and DAPI) in myotubes (i.e., cells with $\geq 3$ nuclei) were counted. The sum of five images was used to represent each well.

\section{Luciferase detection}

Cell monolayers of Cre and LV-floxed-Luc C2C12 co-cultures were lysed in reporter lysis buffer (Promega, Madison, WI) by scraping and a subsequent freeze-thaw cycle, and briefly centrifuged to remove cell debris. Luciferase activity was measured by addition of $100 \mu \mathrm{L}$ luciferase assay reagent $\left(1.07 \mathrm{mM} \mathrm{MgCO}_{3^{\prime}} 2.67 \mathrm{mM} \mathrm{MgSO}_{4^{\prime}}, 20.0 \mathrm{mM}\right.$ Tricin, $0.10 \mathrm{mM}$ EDTA, $33.3 \mathrm{mM}$ DTT, $530 \mu \mathrm{M}$ ATP, $270 \mu \mathrm{M}$ Coenzyme A, $470 \mu \mathrm{M}$ Luciferin) to 20 $\mu \mathrm{L}$ lysate, and subsequent luminescence detection using a single tube luminometer (Lumat LB 9507, Berthold, Bad Wildbad, Germany), according to the manufacturer's protocol (Promega). The luminescence signal (RLU) was corrected for total protein content in the soluble fraction assessed by a BCA protein assay (Pierce Biotechnology, Rockford, IL).

\section{Statistics}

Data are presented as means \pm SEM and are representative of $\geq 3$ independent experiments. Analyses were performed using SPSS Statistics (version 22.0, IBM Corp., Armonk, NY). Comparisons between two groups were performed using an independent student's t-test. Comparisons between $>2$ groups were performed by one-way ANOVA with Bonferroni post hoc correction. A p-value $<0.05$ was considered statistically significant. 


\section{RESULTS}

\section{In vitro fusion of myoblasts with myotubes}

The classical in vitro myogenesis model entails the formation of syncytia from myoblasts. To better mimic postnatal myogenesis in vitro, we sought to represent the involved fusion partners. To this end, myotubes obtained by 5-day differentiation of C2C12 myoblasts were co-cultured with yet undifferentiated myoblasts. Through live cell time-lapse imaging fusion of myoblasts with myotubes was observed during the $48 \mathrm{~h}$ after initiation of co-culturing (Figure S1; Additional File 1). Accordingly, the fusion of DiO-stained C2C12 myoblasts with DiD-stained myotubes resulted in the formation of hybrid myotubes (Figure 1), and in vitro myotube-myoblast fusion was confirmed in a similar experiment in HSM cells (Figure S2). Together, this shows that both $\mathrm{C} 2 \mathrm{C} 12$ and HSM cells are capable of in vitro postnatal myonuclear accretion.

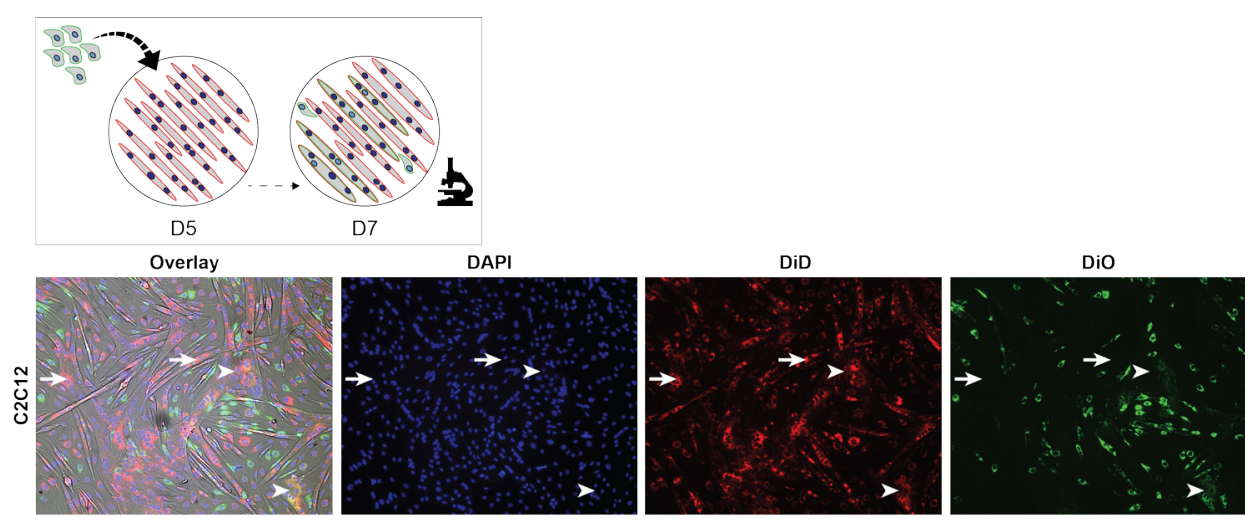

Figure 1. In vitro myoblast-myotube fusion. Hybrid formation in DiD-stained C2C12 myotubes 2 days after initiation of co-culturing with DiO-stained C2C12 myoblasts. (DAPI/nuclei: blue; DiD: red; DiO: green). Arrows indicate non-hybrid myotubes, arrow heads indicate hybrid myotubes.

\section{In vitro postnatal myonuclear accretion is increased by IGF-I}

Staining-based quantification was optimized (Figure S3), and used to assess if the number of in vitro postnatal myonuclear accretion events can be modified. Co-cultures were treated with IGF-I, representing a well-established myogenic factor, which impacts on both proliferation and differentiation [28]. This revealed a higher total amount of myotubes, a higher total amount of hybrids, and a higher relative amount of hybrids 2 days after initiation of co-culturing in the presence of IGF-I (Figure 2A-C). IGF-I treatment started $24 \mathrm{~h}$ after initiation of co-culturing had no effect, whereas $24-\mathrm{h}$ pre-treatment with IGF-I increased the number of myotubes but did not affect the relative amount of hybrid myotubes (Figure S4). This showed that the staining-based 


\section{CHAPTER 6}

method had sufficient power to detect relevant differences in postnatal myonuclear accretion. Furthermore, the staining-based method displayed a significant inter-rater correlation and a moderate to high inter-rater agreement (Figure S5). However, BlandAltman analysis revealed a significant fixed bias for both the absolute and relative amount of hybrids, and potentially clinically relevant differences may lie within the 95\% limits of agreement (Figure S5D, F). Moreover, the staining-based assessment of postnatal myonuclear accretion was labor intensive and time consuming. For unbiased, high throughput, semi-quantitative assessment of postnatal myonuclear accretion, we therefore developed a Cre/LoxP-based cell fusion reporter system (Figure S6), which allows the conditional expression of luciferase after myoblast-myotube fusion. IGF-I treatment of LV-floxed-Luc myotubes and Cre myoblast co-cultures increased protein content and absolute luciferase activity, but no change in the relative luciferase activity was observed. However, IGF-I treatment of Cre myotube and LV-floxed-Luc myoblast co-cultures resulted in an increased protein content, and increased relative and absolute luciferase activity in cells lysed 3 days after initiation of co-culturing (Figure 2D-F, Figure S6F-H), indicating increased cell fusion.

The Cre/LoxP-based cells system was further characterized by employing cocultures with increasing concentrations of IGF-I. A dose-dependent increase in the relative luciferase activity and total protein content was observed upon treatment with increasing concentrations of IGF-I (Figure S7D-F). To verify IGF-I stimulated myonuclear accretion, the incorporation of BrdU-labelled myoblast nuclei into myotubes was assessed. A dose-dependent increase in the number of $\mathrm{BrdU}^{+}$nuclei in myotubes was observed upon treatment with IGF-I, which had a strong, but nonsignificant correlation with the change in relative luciferase activity (Figure S7G-I). To further validate luciferase activity in the Cre/LoxP-based model as an index of fusion, the stimulatory effects of IGF-I on protein synthesis- and fusion-dependent luciferase expression were dissected. Cre and LV-floxed-Luc myoblasts were co-cultured and differentiated into hybrid myotubes with constitutive luciferase expression, and their subsequent time- and dose-dependent luciferase activity in response to IGF-I was compared to myoblast-myotube co-cultures (Figure S8). Although IGF-I treatment increased the relative luciferase activity at $T=72$ in both systems, the magnitude of the effect in the preformed hybrid myotubes was negligible compared to that observed in fusion-dependent luciferase expressing co-cultures (Figure S8). 

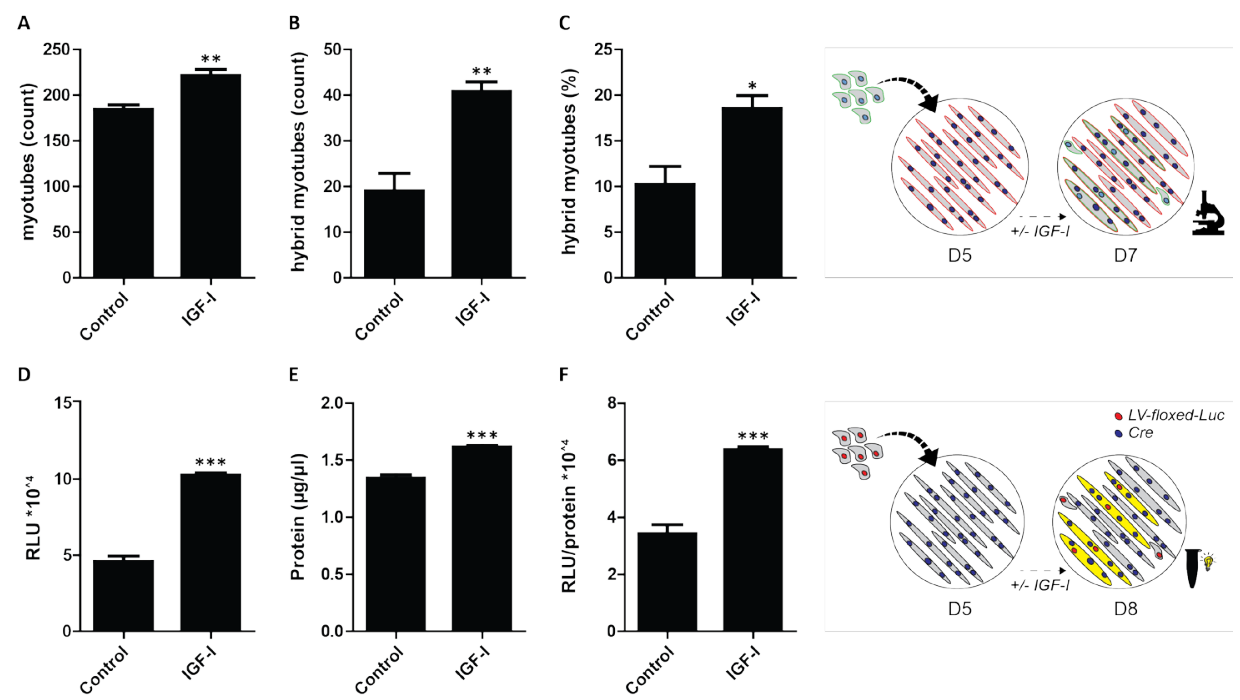

Figure 2. Increased in vitro postnatal myonuclear accretion in C2C12 cells upon IGF-I treatment. (AC) Staining-based assessment of myonuclear accretion 2 days after initiation of co-culturing $+/-10$ nM IGF-I. A) total number of myotubes, B) number of hybrid myotubes, C) \% hybrid myotubes. (D-F) Luciferase-based assessment of myonuclear accretion 3 days after initiation of co-culturing +/- 10 $n M$ IGF-I. D) luciferase activity ( $R L U)$ per well, E) protein content $(\mu \mathrm{g} / \mu \mathrm{L})$ per well, F) relative luciferase activity (RLU/protein content) per well. Values are means \pm SEM, $n=4 .{ }^{*} p<0.05,{ }^{* *} p<0.01,{ }^{* * *} p<0.001$.

\section{In vitro postnatal myonuclear accretion is reduced by Cytochalasin D, Myostatin and TNFa}

To assess if the rate of in vitro postnatal myonuclear accretion can be suppressed, cocultures were treated with known inhibitors of myogenesis: CytoD, MSTN and TNFa. CytoD treatment upon initiation of co-culturing resulted in a reduction of luciferase activity to background levels measured in myotube only cultures, and a small increase in total protein content (Figure 3A-C). MSTN treatment upon initiation of co-culturing had no effect on the protein content, but led to a decrease in absolute and relative fusion reporter activity (Figure 3D-F). Similarly, TNFa treatment upon initiation of coculturing led to a decrease in absolute and relative fusion reporter activity, in presence of an increase in the total protein content per well (Figure 3G-I). Suppression of fusion reporter activity by TNFa treatment was dose-dependent (Figure S7A-C). Moreover, when applied to preformed hybrid myotube cultures no decrease in absolute or negative luciferase content was detected in response to TNFa (Figure S8D-F), suggesting reduced fusion-dependent luciferase expression in myoblast-myotube cocultures (Figure $3 \mathrm{G}-\mathrm{I}$ ) rather than decreased synthesis of luciferase protein. 


\section{CHAPTER 6}

A

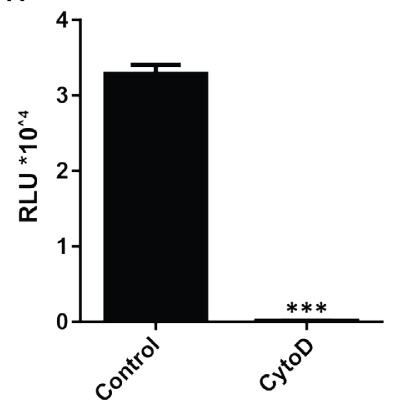

D

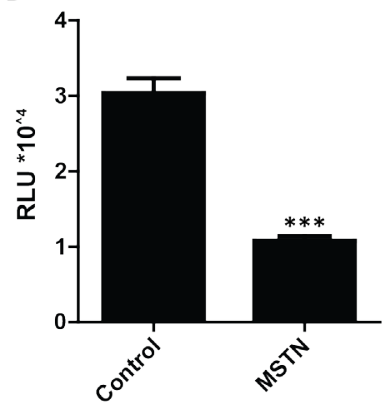

G

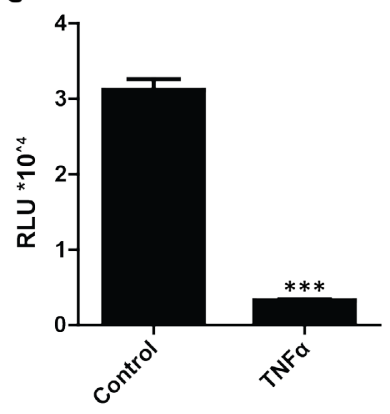

B

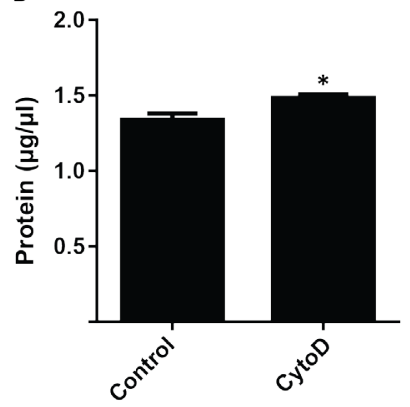

E

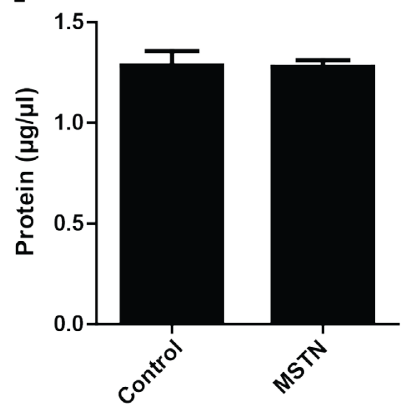

H

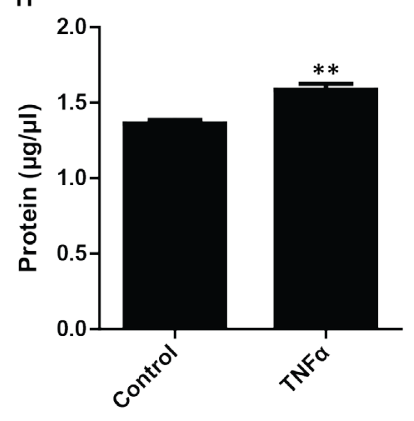

C

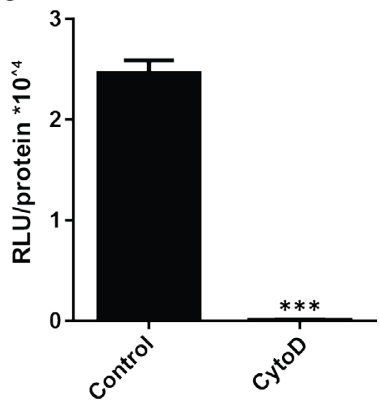

$\mathbf{F}$

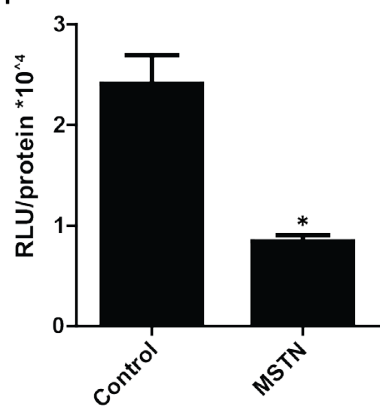

I

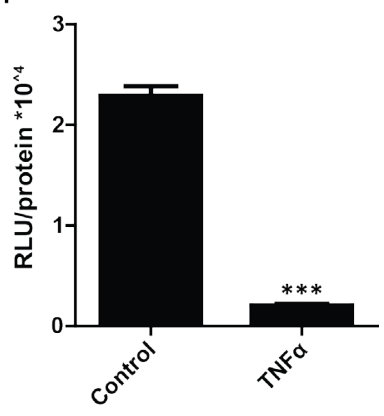

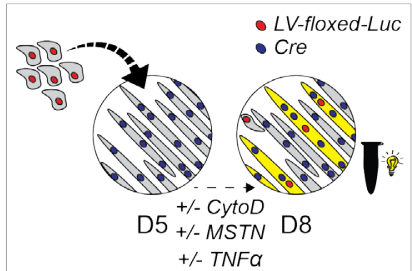

Figure 3. | Decreased in vitro postnatal myonuclear accretion in C2C12 cells upon CytoD, MSTN and TNFa treatment. Luciferase-based assessment of myonuclear accretion 3 days after initiation of coculturing +/- $0.3 \mu M$ CytoD (A-C), $250 \mathrm{ng} / \mathrm{mL}$ MSTN (D-F), or $10 \mathrm{ng} / \mathrm{mL} T \mathrm{NFa}(\mathrm{G}-\mathrm{I})$. A) luciferase activity $(R L U)$ per well, $B)$ protein content $(\mu \mathrm{g} / \mu \mathrm{L})$ per well, C) relative luciferase activity (RLU/protein content) per well, D) luciferase activity $(R L U)$ per well, E) protein content $(\mu \mathrm{g} / \mu \mathrm{L})$ per well, F) relative luciferase activity (RLU/protein content) per well, G) luciferase activity ( $R L U)$ per well, $H$ ) protein content $(\mu \mathrm{g} / \mu \mathrm{L})$ per well, I) relative luciferase activity (RLU/protein content) per well. Values are means $\pm S E M, n=4$. ${ }^{*} p<0.05,{ }^{* *} p<0.01,{ }^{* * *} p<0.001$. 


\section{In vitro postnatal myonuclear accretion is increased by the anti-inflammatory cytokines IL-13 and IL-4.}

In contrast to the pro-inflammatory cytokine TNFa, the anti-inflammatory cytokines IL-13 and IL-4 have been identified as promoting factors for postnatal myogenesis [29, 30]. IL-13 treatment upon initiation of co-culturing resulted in an increase in the total protein content and an increase in the absolute and relative fusion reporter activity (Figure 4A-C). Similarly, IL-4 treatment tended to increase the total protein content and led to an increase in the absolute and relative fusion reporter activity (Figure 4DF).

A

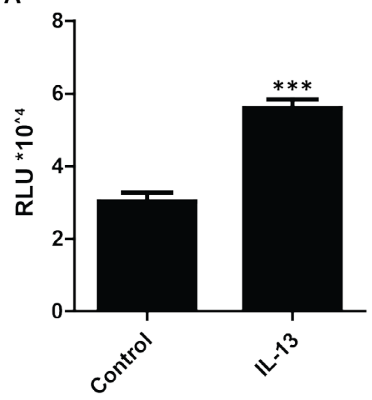

D

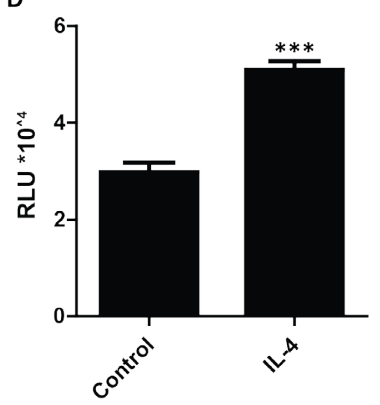

B

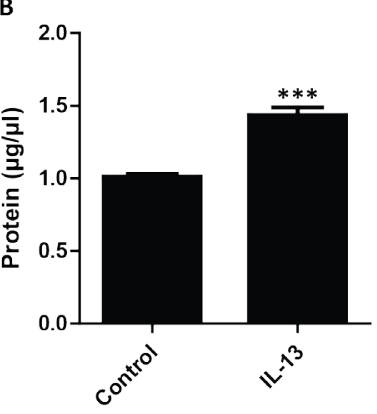

$\mathrm{E}$
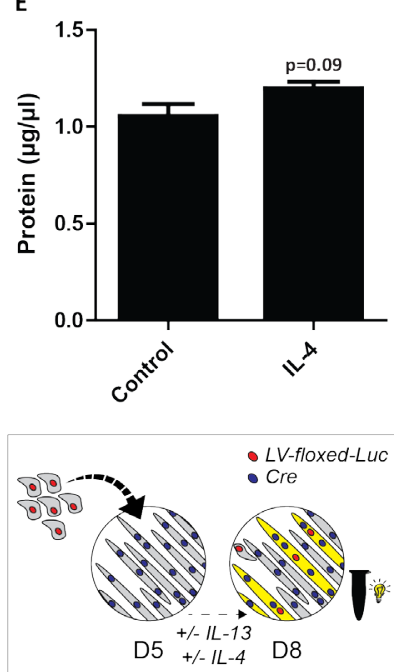

C

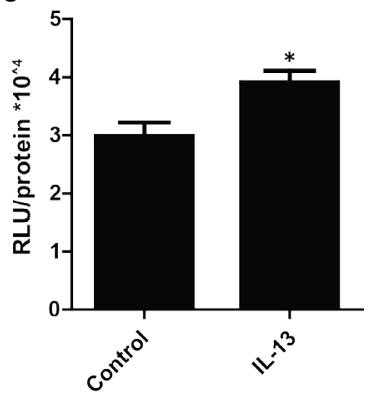

$\mathbf{F}$

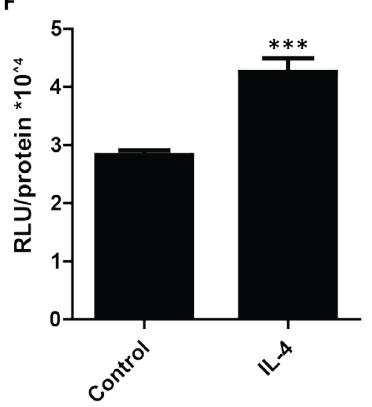

Figure 4. | Increased in vitro postnatal myonuclear accretion in C2C12 cells upon IL-13 and IL-4 treatment. Luciferase-based assessment of myonuclear accretion 3 days after initiation of coculturing +/- $250 \mathrm{ng} / \mathrm{mL} I L-13(A-C)$ or $500 \mathrm{ng} / \mathrm{mL} I L-4$ (D-F). A) luciferase activity (RLU) per well, B) protein content $(\mu \mathrm{g} / \mu \mathrm{L})$ per well, C) relative luciferase activity (RLU/protein content) per well, D) luciferase activity (RLU) per well, E) protein content $(\mu \mathrm{g} / \mu \mathrm{L})$ per well, F) relative luciferase activity (RLU/protein content) per well. Values are means \pm SEM, $n=4 .{ }^{*} p<0.05,{ }^{* * *} p<0.001$. 


\section{In vitro postnatal myonuclear accretion is increased upon recovery from myotube injury and atrophy}

Physiological conditions that trigger in vivo postnatal myonuclear accretion include the recovery from muscle damaging exercise such as eccentric exercise and the recovery from muscle atrophy. In vitro myotube damage induced by electrical pulse stimulation (EPS) followed by recovery in the presence of myoblasts led to an increased total protein content and increased absolute and relative fusion reporter activity compared to untreated co-cultures (Figure $5 \mathrm{~A}-\mathrm{C}$ ). In vitro induction of atrophy by LY pre-treatment and maintenance during co-culturing led to a significant decrease in the total protein content and a decrease in the absolute and relative fusion reporter activity (Figure 5D-F). Furthermore, removal of LY upon initiation of co-culturing led to a normalization of the total protein content and an increase in the absolute and relative fusion reporter activity compared to maintained LY stimulation and control (Figure 5D-F).
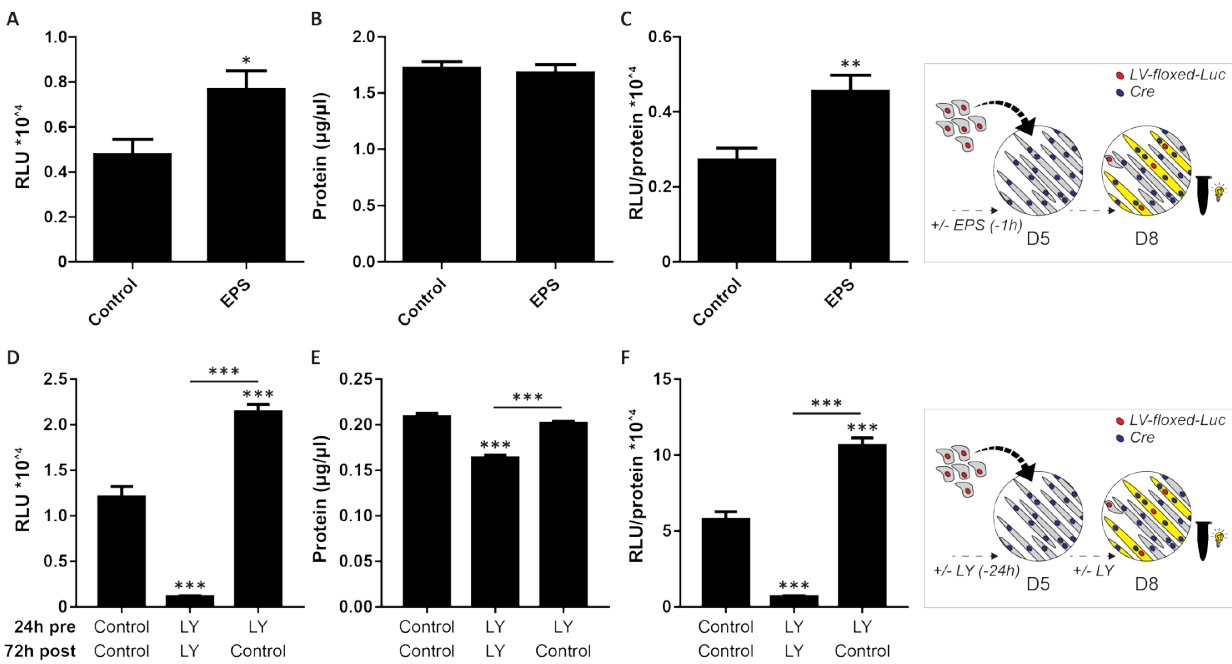

Figure 5. | Increased in vitro postnatal myonuclear accretion in C2C12 cells upon recovery from myotube damage and atrophy. Luciferase-based assessment of myonuclear accretion 3 days after initiation of co-culturing of myotubes pre-treated by 1-hour electrical pulse stimulation, with myoblasts in presence of conditioned medium ( $n=6)$. A) luciferase activity (RLU) per well, B) protein content $(\mu \mathrm{g} / \mu \mathrm{L})$ per well, C) relative luciferase activity (RLU/protein content) per well. Luciferasebased assessment of myonuclear accretion 3 days after initiation of co-culturing of myotubes pre-treated +/- $15 \mu M L Y$ (24h pre), with myoblasts in presence or absence of $15 \mu M L Y$ (72h post) $(n=4)$. D) luciferase activity ( $R L U)$ per well, E) protein content $(\mu \mathrm{g} / \mu \mathrm{L})$ per well, F) relative luciferase activity (RLU/protein content) per well. T=initiation and duration of treatment $(h)$ relative to start of co-culturing. Values are means $\pm S E M, n=4$. ${ }^{*} p<0.05,{ }^{* *} p<0.01,{ }^{* * *} p<0.001$ compared to control or between indicated groups. 


\section{In vitro postnatal myonuclear accretion requires the expression of Myomaker in myoblasts}

A novel factor involved in myogenic fusion that is required for muscle regeneration and hypertrophy is Myomaker [15, 31]. A cell type-specific differential requirement of Myomaker has been reported [32]. We assessed if the requirement of Myomaker for fusion is myoblast or myotube specific. SiRNA-mediated knockdown of Myomaker was verified and resulted in a strong reduction in Myomaker protein abundance in myotubes, whereas Myomaker expression was below the detection limit in myoblasts (Figure 6D-E). Knockdown of Myomaker in myotubes did not alter the absolute and relative cell fusion reporter activity in co-cultures (Figure $6 \mathrm{~A}-\mathrm{C}$ ). In contrast, treatment of myoblasts with siRNA against Myomaker decreased both the absolute and relative fusion reporter activity in co-cultures containing Myomaker-expressing or -silenced myotubes (Figure $6 \mathrm{~A}-\mathrm{C}$ ). These data indicate that Myomaker expression in myoblasts is required for cell fusion.
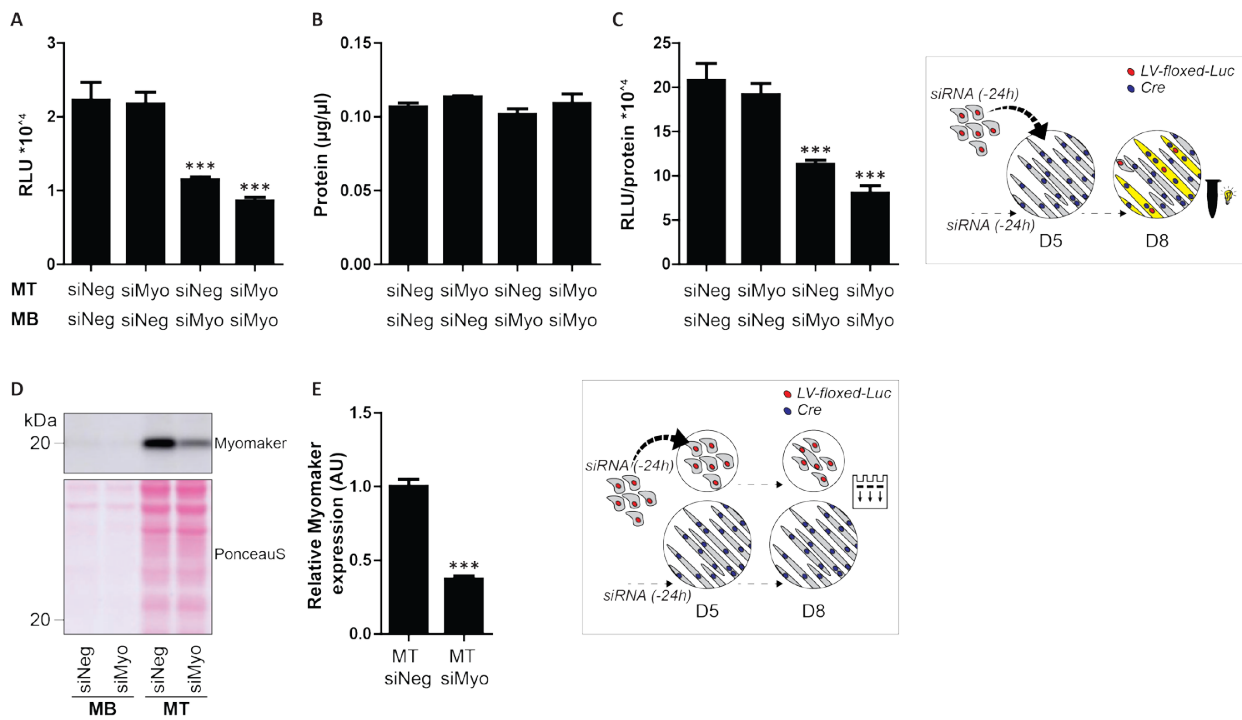

Figure 6. | Myomaker is specifically required in myoblasts for in vitro postnatal myonuclear accretion in C2C12 cells. Luciferase-based assessment of myonuclear accretion 3 days after initiation of coculturing of myotubes (MT) with myoblasts (MB) which received 24-hour pre-treatment with either negative control siRNA (siNeg) or siRNA against Myomaker (siMyo) $(n=4)$. A) luciferase activity (RLU) per well, B) protein content ( $\mu \mathrm{g} / \mu \mathrm{L})$ per well, C) relative luciferase activity (RLU/protein content) per well. Parallel experiment of siRNA treated MT and MB, maintained in separate cultures. D) Western blot of Myomaker, E) densitometric analysis of $20 \mathrm{kDa}$ band $(n=8)$. Values are means \pm SEM. ${ }^{* * *} p<0.001$ compared to control. 


\section{CHAPTER 6}

\section{DISCUSSION}

The requirement for postnatal myogenesis in muscle growth, repair and regeneration, and basal muscle maintenance is evident from research in humans [8, 33, 34] and rodents $[9-12,35]$. However, data on the regulation and dysregulation of postnatal myonuclear accretion is scarce due to the technical challenge to investigate and quantitate this process in vivo and in vitro. Although, myoblast-myotube fusion is frequently mentioned in literature, the majority of these in vitro studies actually refer to late myogenesis in progressively differentiating cultures. Certainly, these classical studies have provided invaluable insights in the regulation and dysregulation of myogenesis, however, their translatability to adult skeletal muscle maintenance can be challenged [5, 22-24].

In the current study, we developed an unbiased, high throughput, Cre/LoxP-based in vitro model system for semi-quantitative assessment of postnatal myonuclear accretion. This model builds on the broadly employed classical model of myogenesis, by including the fusion partners involved in in vivo postnatal myonuclear accretion through co-culturing of differentiated $\mathrm{C} 2 \mathrm{C} 12$ myotubes with yet undifferentiated C2C12 myoblasts. Via live cell time-lapse imaging, staining-based cell tracing, and recombination-dependent luciferase activity, we show that postnatal myonuclear accretion occurs in vitro. This finding is in line with the scant previous studies assessing myoblast-myotube fusion in co-cultures by staining-based cell tracing [30, 36-39]. Furthermore, we reproduced this finding in a HSM cell line, and thereby confirm that in vitro myoblast-myotube fusion is not a $\mathrm{C} 2 \mathrm{C} 12$ cell line specific anomaly.

Using this co-culture model, a substantial number of hybrid cells and a pronounced luciferase signal are detected even in the absence of an additional myogenic trigger. Although previous literature states that in uninjured adult muscles, satellite cells are mitotically quiescent [12], in line with our data, a more recent study challenged this proposed low basal myonuclear turnover rate and shows that a significant percentage of satellite cells divide and fuse in uninjured adult muscles [40]. Differences in observed 'basal' myonuclear turnover rates may arise from variations in the employed methodologies. Importantly, satellite cells were reported to rapidly activate in explant cultures [41], which resembles the proliferative state of myoblasts in our co-culture model.

Based on the basal luciferase signal and the responsiveness of the Cre/LoxP-based reporter system with Cre and LV-floxed-Luc C2C12 alternating as acceptor and donor 
cells for myonuclear accretion, we conclude that co-culturing of Cre myotubes with LV-floxed-Luc myoblasts most sensitively reports fusion (Figure S6). This likely reflects the recombination-dependent increase in luciferase expression in Cre myotubes with the incorporation of every additional nucleus derived from an LV-floxed-Luc myoblast, whereas accretion of one single nucleus derived from a Cre myoblast may result in the recombination of multiple nuclei present in one LV-floxed-Luc myotube. We show that in this model, IGF-I induces a profound increase in postnatal myonuclear accretion. IGF-I is a well-established driver of muscle hypertrophy that has been associated with increases in both protein synthesis and myogenesis [42]. In line with this, IGF-I treatment during co-culturing increases the total protein content, as well as cell fusion assessed by staining-based and reporter-based fusion quantification. In agreement with previous literature [42], the effect of IGF-I depends on the timing of treatment initiation, which may suggest a role for temporal IGF-I expression during postnatal myogenesis. Furthermore, we show that TNFa treatment decreases postnatal myonuclear accretion. This disease-related factor was previously shown to reduce the myogenic index [19]. Although we demonstrate a dose-dependent decrease in fusion upon increasing TNFa concentrations, a bimodal effect of TNFa treatment was found when lower concentrations were included [43]. Furthermore, positive effects of TNFa on proliferation have been reported [44], which may explain the increase in total protein content in TNFa-treated co-cultures.

These modulatory effects of IGF-I and TNFa show that the Cre/LoxP-based fusion reporter system is responsive to physiologically relevant ligands that modulate myogenesis. Furthermore, the IGF-I and TNFa-dose dependent (Figure S7A-F), and the myoblast-density dependent (Figure S6E) effects on the relative luciferase activity, together with the lag time for effects of modulations to result in changes in luciferase activity (Figure $\mathrm{S} 6 \mathrm{~F}-\mathrm{H}$, Figure $\mathrm{S} 8 \mathrm{~A}-\mathrm{C}$ ), suggest that the relative luciferase activity is reflecting fusion. Moreover, in keeping with previous research [32, 45], treatment of co-cultures with CytoD reduces the luciferase reporter activity to background levels, verifying that luciferase reporter activity in our system requires cell fusion. In line, the stimulation of luciferase activity corresponds with an increased presence of myoblastderived myonuclei in myotubes, providing further support that alterations in luciferase activity are determined by, and therefore reflect fusion. Nevertheless, the absolute luciferase activity is a product of post-recombinational luciferase expression, which may be affected by alterations in cellular transcriptional and translational activity. To disentangle effects on fusion and protein synthesis, we compared the time-dependent effects of IGF-I and TNFa on fusion-dependent luciferase expressing co-cultures, and Cre and LV-floxed-Luc hybrid myotubes with constitutive luciferase expression, 


\section{CHAPTER 6}

respectively (Figure S8). Based on the large difference in the magnitude of the effect of IGF-I, and the difference in the direction of the effect of TNFa on the relative luciferase activity, we conclude that changes in the relative luciferase activity in the Cre/LoxPbased fusion reporter system are indeed mainly dictated by changes in fusion. Nevertheless, when the absolute rate of myonuclear accretion is of interest, luciferasebased screening should be followed up by quantitative assessment of myonuclear accretion, e.g. by staining-based nuclear tracing of BrdU labelled myoblasts.

The current method reports the cumulative functional contribution of fusion events, and may thereby facilitate the elucidation of the homeostatic control of myonuclear number, which is of particular interest for the slow progressive muscle wasting seen in aging [8], chronic diseases [46], and muscle dystrophy [47]. However, luciferase-based reporting of fusion operates with a certain lag time, determined by recombination, transcription, and translation of the reporter gene. Therefore, no complete distinction can be made between alterations in the amount or rate of myonuclear accretion, although both may be equally relevant. To gain a more mechanistic insight in the regulation of postnatal myonuclear accretion, the current method should be used in conjunction with assays for proliferation and biochemical differentiation, to distinguish between effects on those processes and fusion, as alterations in any of these processes will affect myonuclear accretion. Furthermore, it should be taken into account that long term monitoring of in vitro myonuclear accretion is restricted by the limited cell viability in culture. Moreover, in contrast to the staining-based assessment of in vitro postnatal myonuclear accretion, Cre/LoxP-based assessment does not provide any insight in the intermediate morphological states of myoblasts before fusion with a myotube. Thus, although we refer to myoblast-myotube fusion, we cannot exclude the occurrence nor assess the relevance of e.g. myoblast-myoblast fusion before fusion with a pre-existing myotube. Nevertheless, a major advantage of the Cre/LoxP-based system is that it does not rely on visual identification of individual myotubes which is labor intensive and complex due to irregular myotube structure, and it may therefore also be suitable to study postnatal myonuclear accretion in e.g. 3D culture systems [48].

In an initial utilization of the model, we assessed pharmacological and physiological modulators of postnatal myonuclear accretion. Similar to IGF-I [49, 50], MSTN modulation is part of the exercise recovery response [51,52]. Treatment of co-cultures with the myogenic inhibitor MSTN results in a pronounced reduction of cell fusion, which is in agreement with previous studies that show a reduction in the myogenic index by treatment with MSTN $[21,53]$. Furthermore, cytokine secretion is induced 
during physiological conditions that are accompanied by myonuclear accretion [52, 54], such as recovery from eccentric exercise and recovery from muscle atrophy. In contrast to the inhibitory effect of the pro-inflammatory cytokine TNFa on myogenesis, the anti-inflammatory cytokines IL-13 and IL-4 have been reported to increase fusion by acting as recruitment factors for muscle growth [29, 30]. In line with this, we find an increase in the relative fusion reporter activity upon co-culture treatment with IL-4 and IL-13. Furthermore, during the recovery phase after simulation of muscle damaging exercise in vitro through electrical pulse stimulation [27], we observe an increase in myonuclear accretion. Moreover, upon induction of in vitro muscle atrophy by treatment with the Pi3K inhibitor LY [55], in line with previous research [56], a reduction in myonuclear accretion in the presence of $L Y$ is observed, which may reflect a requirement of IGF-I/AKT signaling in one of the myogenic processes leading to myonuclear accretion. Interestingly, myonuclear accretion is increased during recovery from myotube atrophy upon removal of $L Y$, illustrating that myonuclear accretion can be determined by myotube intrinsic properties, and suggesting a role of myonuclear accretion in restoring myofiber size.

In vivo, contraction-induced muscle damage and muscle atrophy are processes that affect the adult myofiber. However, the subsequent satellite cell response during muscle recovery suggests that crosstalk occurs between muscle fibers and satellite cells. Importantly, the current model system allows the separate treatment of myotubes and myoblasts, and thereby provides the possibility to disentangle cell type-specific signals and responses, and facilitates exploration of their role in the regulation of postnatal myogenesis. We exploited this distinctive capacity by assessing whether Myomaker, a recently identified regulator of fusion, is required for postnatal myonuclear accretion in a cell-type specific manner. To this end, we knocked down Myomaker in myotubes and/or myoblasts before initiation of co-culturing, and report a myoblast specific requirement of Myomaker for postnatal myonuclear accretion. This is in line with an early Myomaker study, which reported a cell-type specific differential requirement of this protein in myoblasts-fibroblasts fusion [32], and a more recent study, which reported a satellite cell-specific requirement for Myomaker in overloadinduced muscle hypertrophy [15].

\section{CONCLUSIONS}

In summary, we developed a Cre/LoxP-based in vitro model that better reflects postnatal myogenesis by including the relevant fusion partners, and can be used for semi-quantitative assessment of postnatal myonuclear accretion. We show that 


\section{CHAPTER 6}

this model is responsive to known ligands with myogenesis inducing and inhibiting properties, as well as physiological triggers that affect postnatal myonuclear accretion. Moreover, this model allows the distinction the cell type-specific roles of signals and responses in the regulation of postnatal myogenesis. Together, these features make the Cre/LoxP-based in vitro model of postnatal myonuclear accretion suitable for both basal research on the molecular regulation of postnatal myogenesis, as well as translational research on the whole range of muscle diseases that may involve impaired satellite cell number or function. 


\section{REFERENCES}

1. Buckingham $M$, Bajard $L, C h a n g ~ T$, et al. The formation of skeletal muscle: from somite to limb. Journal of anatomy 2003;202(1):59-68.

2. Relaix F, Rocancourt D, Mansouri A, et al. A Pax3/Pax7-dependent population of skeletal muscle progenitor cells. Nature 2005;435(7044):948-53.

3. Gros J, Manceau M, Thome $V$, et al. A common somitic origin for embryonic muscle progenitors and satellite cells. Nature 2005;435(7044):954-8.

4. Kassar-Duchossoy L, Giacone E, Gayraud-Morel B, et al. Pax3/Pax7 mark a novel population of primitive myogenic cells during development. Genes \& development 2005;19(12):142631.

5. Hutcheson DA, Zhao J, Merrell A, et al. Embryonic and fetal limb myogenic cells are derived from developmentally distinct progenitors and have different requirements for betacatenin. Genes \& development 2009;23(8):997-1013.

6. Davis TA, Fiorotto ML. Regulation of muscle growth in neonates. Current opinion in clinical nutrition and metabolic care 2009;12(1):78-85.

7. Dhawan J, Rando TA. Stem cells in postnatal myogenesis: molecular mechanisms of satellite cell quiescence, activation and replenishment. Trends in cell biology 2005;15(12):666-73.

8. Snijders T, Nederveen JP, McKay BR, et al. Satellite cells in human skeletal muscle plasticity. Frontiers in physiology 2015;6:283.

9. Rosenblatt JD, Parry DJ. Gamma irradiation prevents compensatory hypertrophy of overloaded mouse extensor digitorum longus muscle. Journal of applied physiology 1992;73(6):2538-43.

10. Rosenblatt JD, Parry DJ. Adaptation of rat extensor digitorum longus muscle to gamma irradiation and overload. Pflugers Archiv : European journal of physiology 1993;423(34):255-64.

11. Fry $C S$, Lee $J D$, Jackson $J R$, et al. Regulation of the muscle fiber microenvironment by activated satellite cells during hypertrophy. FASEB journal : official publication of the Federation of American Societies for Experimental Biology 2014;28(4):1654-65.

12. Relaix F, Zammit PS. Satellite cells are essential for skeletal muscle regeneration: the cell on the edge returns centre stage. Development 2012;139(16):2845-56.

13. McCarthy JJ, Mula J, Miyazaki M, et al. Effective fiber hypertrophy in satellite cell-depleted skeletal muscle. Development 2011;138(17):3657-66.

14. Egner IM, Bruusgaard JC, Gundersen K. Satellite cell depletion prevents fiber hypertrophy in skeletal muscle. Development 2016;143(16):2898-906.

15. Goh Q, Millay DP. Requirement of myomaker-mediated stem cell fusion for skeletal muscle hypertrophy. Elife 2017;6

16. Hawke TJ, Garry DJ. Myogenic satellite cells: physiology to molecular biology. Journal of applied physiology 2001;91(2):534-51.

17. Theriault $M E$, Pare $M E$, Lemire $B B$, et al. Regenerative defect in vastus lateralis muscle of patients with chronic obstructive pulmonary disease. Respir Res 2014;15:35.

18. Brack AS, Rando TA. Intrinsic changes and extrinsic influences of myogenic stem cell function during aging. Stem Cell Rev 2007;3(3):226-37.

19. Langen $\mathrm{RC}$, Schols $A M$, Kelders $M C$, et al. Inflammatory cytokines inhibit myogenic differentiation through activation of nuclearfactor-kappaB.FASEBjournal:official publication of the Federation of American Societies for Experimental Biology 2001;15(7):1169-80.

20. Rochat $A$, Fernandez $A$, Vandromme $M$, et al. Insulin and wnt1 pathways cooperate to induce reserve cell activation in differentiation and myotube hypertrophy. Mol Biol Cell 


\section{CHAPTER 6}

2004;15(10):4544-55.

21. McFarlane $\mathrm{C}$, Hui GZ, Amanda WZ, et al. Human myostatin negatively regulates human myoblast growth and differentiation. Am J Physiol Cell Physiol 2011;301(1):C195-203.

22. Wang J, Conboy I. Embryonic vs. adult myogenesis: challenging the 'regeneration recapitulates development' paradigm. Journal of molecular cell biology 2010;2(1):1-4.

23. Lepper C, Conway SJ, Fan CM. Adult satellite cells and embryonic muscle progenitors have distinct genetic requirements. Nature 2009;460(7255):627-31.

24. Kang JS, Krauss RS. Muscle stem cells in developmental and regenerative myogenesis. Current opinion in clinical nutrition and metabolic care 2010;13(3):243-8.

25. Tajbakhsh S. Skeletal muscle stem cells in developmental versus regenerative myogenesis. Journal of internal medicine 2009;266(4):372-89.

26. Thorley M, Duguez S, Mazza EM, et al. Skeletal muscle characteristics are preserved in hTERT/cdk4 human myogenic cell lines. Skelet Muscle 2016;6(1):43.

27. Orfanos Z, Godderz MP, Soroka E, et al. Breaking sarcomeres by in vitro exercise. Sci Rep 2016;6:19614.

28. Coolican SA, Samuel DS, Ewton DZ, et al. The mitogenic and myogenic actions of insulin-like growth factors utilize distinct signaling pathways. J Biol Chem 1997;272(10):6653-62.

29. Jacquemin V, Butler-Browne GS, Furling D, et al. IL-13 mediates the recruitment of reserve cells for fusion during IGF-1-induced hypertrophy of human myotubes. J Cell Sci 2007;120(Pt 4):670-81.

30. Horsley V, Jansen KM, Mills ST, et al. IL-4 acts as a myoblast recruitment factor during mammalian muscle growth. Cell 2003;113(4):483-94.

31. Millay DP, Sutherland LB, Bassel-Duby R, et al. Myomaker is essential for muscle regeneration. Genes \& development 2014;28(15):1641-6.

32. Millay DP, O'Rourke JR, Sutherland LB, et al. Myomaker is a membrane activator of myoblast fusion and muscle formation. Nature 2013;499(7458):301-5.

33. Joanisse S, Gillen JB, Bellamy LM, et al. Evidence for the contribution of muscle stem cells to nonhypertrophic skeletal muscle remodeling in humans. FASEB journal : official publication of the Federation of American Societies for Experimental Biology 2013;27(11):4596-605.

34. Joanisse S, McKay BR, Nederveen JP, et al. Satellite cell activity, without expansion, after nonhypertrophic stimuli. Am J Physiol Regul Integr Comp Physiol 2015;309(9):R1101-11.

35. Hardee JP, Puppa MJ, Fix DK, et al. The effect of radiation dose on mouse skeletal muscle remodeling. Radiol Oncol 2014;48(3):247-56.

36. Jansen KM, Pavlath GK. Mannose receptor regulates myoblast motility and muscle growth. J Cell Biol 2006;174(3):403-13.

37. Teng S, Stegner D, Chen Q, et al. Phospholipase D1 facilitates second-phase myoblast fusion and skeletal muscle regeneration. Mol Biol Cell 2015;26(3):506-17.

38. Sohn RL, Huang P, Kawahara G, et al. A role for nephrin, a renal protein, in vertebrate skeletal muscle cell fusion. Proc Natl Acad Sci U S A 2009;106(23):9274-9.

39. Gruenbaum-Cohen $Y$, Harel I, Umansky KB, et al. The actin regulator N-WASp is required for muscle-cell fusion in mice. Proc Natl Acad Sci U S A 2012;109(28):11211-6.

40. Pawlikowski B, Pulliam C, Betta ND, et al. Pervasive satellite cell contribution to uninjured adult muscle fibers. Skelet Muscle 2015;5:42.

41. Siegel AL, Atchison $\mathrm{K}$, Fisher KE, et al. $3 \mathrm{D}$ timelapse analysis of muscle satellite cell motility. Stem cells 2009;27(10):2527-38.

42. Jacquemin $V$, Furling $D$, Bigot $A$, et al. IGF-1 induces human myotube hypertrophy by increasing cell recruitment. Exp Cell Res 2004;299(1):148-58. 
43. Chen $\mathrm{SE}$, Jin $\mathrm{B}$, LiYP.TNF-alpha regulates myogenesis and muscle regeneration by activating p38 MAPK. Am J Physiol Cell Physiol 2007;292(5):C1660-71.

44. Li YP. TNF-alpha is a mitogen in skeletal muscle. Am J Physiol Cell Physiol 2003;285(2):C3706.

45. Peckham M. Engineering a multi-nucleated myotube, the role of the actin cytoskeleton. J Microsc 2008;231(3):486-93.

46. Sanders KJ, Kneppers AE, van de Bool C, et al. Cachexia in chronic obstructive pulmonary disease: new insights and therapeutic perspective. J Cachexia Sarcopenia Muscle 2016;7(1):5-22.

47. Blau HM, Webster C, Pavlath GK. Defective myoblasts identified in Duchenne muscular dystrophy. Proc Natl Acad Sci U S A 1983;80(15):4856-60.

48. Afshar Bakooshli M, Gilbert PM. Muscling in on the third dimension. Elife 2015;4:e06430.

49. Sakuma K, Watanabe K, Sano M, et al. Postnatal profiles of myogenic regulatory factors and the receptors of TGF-beta 2, LIF and IGF-I in the gastrocnemius and rectus femoris muscles of dy mouse. Acta Neuropathol 2000;99(2):169-76.

50. Nindl BC, Alemany JA, Tuckow AP, et al. Effects of exercise mode and duration on 24-h IGF-I system recovery responses. Medicine and science in sports and exercise 2009;41(6):126170.

51. Snijders T, Verdijk LB, McKay BR, et al. Acute dietary protein intake restriction is associated with changes in myostatin expression after a single bout of resistance exercise in healthy young men. J Nutr 2014;144(2):137-45.

52. Louis $E$, Raue $U$, Yang $Y$, et al. Time course of proteolytic, cytokine, and myostatin gene expression after acute exercise in human skeletal muscle. Journal of applied physiology 2007;103(5):1744-51.

53. Nozaki M, Li Y, Zhu J, et al. Improved muscle healing after contusion injury by the inhibitory effect of suramin on myostatin, a negative regulator of muscle growth. Am J Sports Med 2008;36(12):2354-62.

54. Petersen AM, Pedersen BK. The anti-inflammatory effect of exercise. Journal of applied physiology 2005;98(4):1154-62.

55. Verhees KJ, Schols AM, Kelders MC, et al. Glycogen synthase kinase-3beta is required for the induction of skeletal muscle atrophy. Am J Physiol Cell Physiol 2011;301(5):C995-C1007.

56. Hu SY, Tai CC, Li YH, et al. Progranulin compensates for blocked IGF-1 signaling to promote myotube hypertrophy in C2C12 myoblasts via the PI3K/Akt/mTOR pathway. FEBS Lett 2012;586(19):3485-92. 


\section{CHAPTER 6}

\section{SUPPLEMENTARY MATERIAL}

Additional File 1. | Live cell time-lapse imaging. In vitro myoblast-myotube fusion captured by live cell time-lapse imaging. Days and hh:mm indicate time after initiation of myotube-myoblast co-culture. https://doi.org/10.6084/m9.figshare.5891122.v1
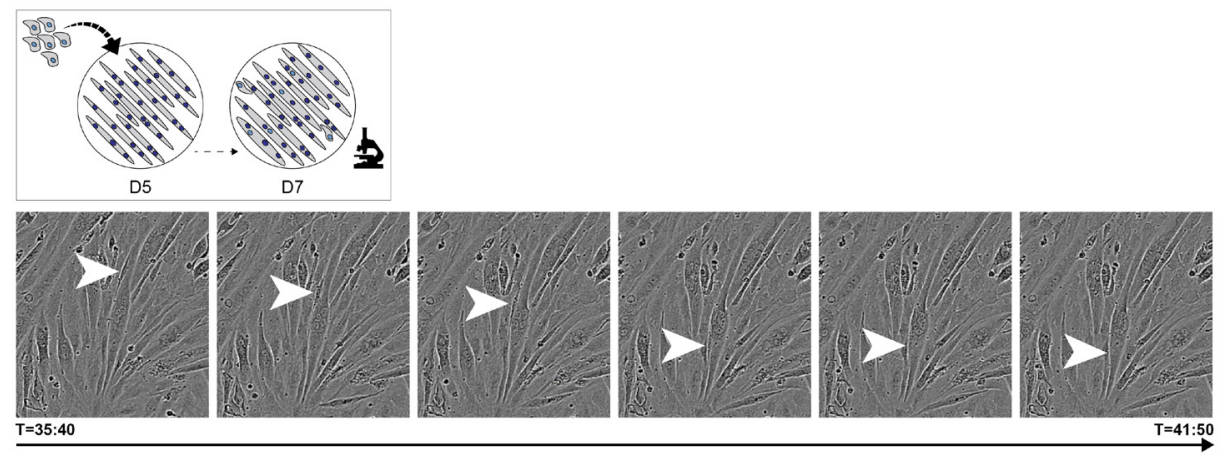

Figure S1. | Selected frames depicting in vitro myoblast-myotube fusion captured by live cell timelapse imaging. Arrow head indicates myoblast movement, and subsequent fusion with a myotube. $T=$ time (hh:mm) after initiation of myotube-myoblast co-culture.
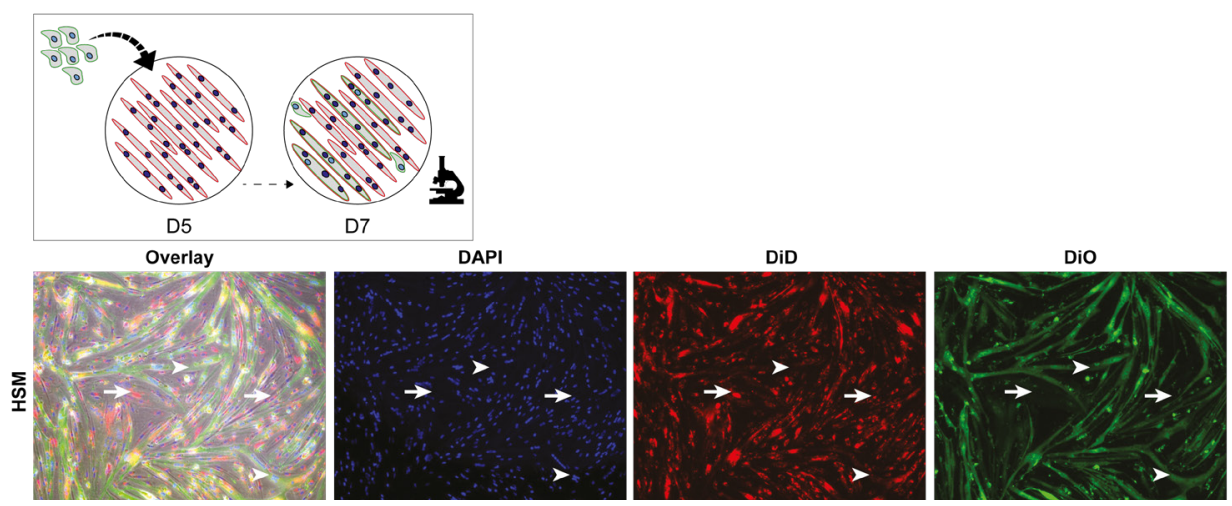

Figure S2. | Hybrid formation in DiD-stained HSM myotubes 2 days after initiation of co-culturing with DiO-stained HSM myoblasts. (DAPI/nuclei: blue; DiD: red; DiO: green). Arrows indicate nonhybrid myotubes, arrow heads indicate hybrid myotubes. 
A

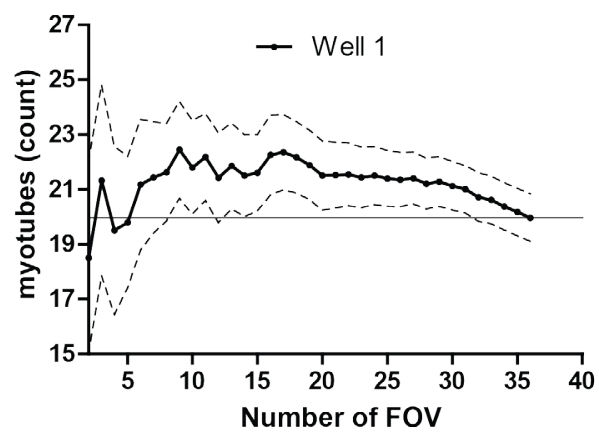

B

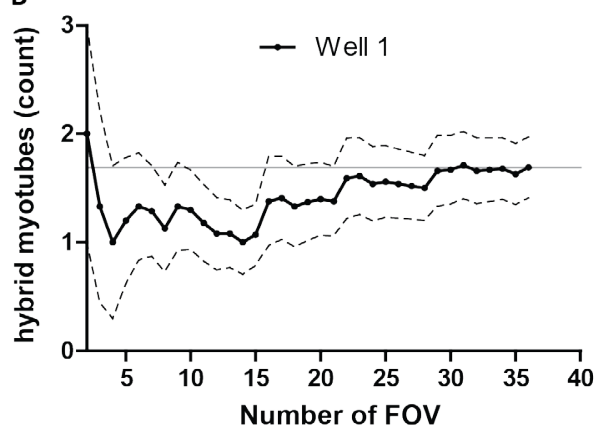

C
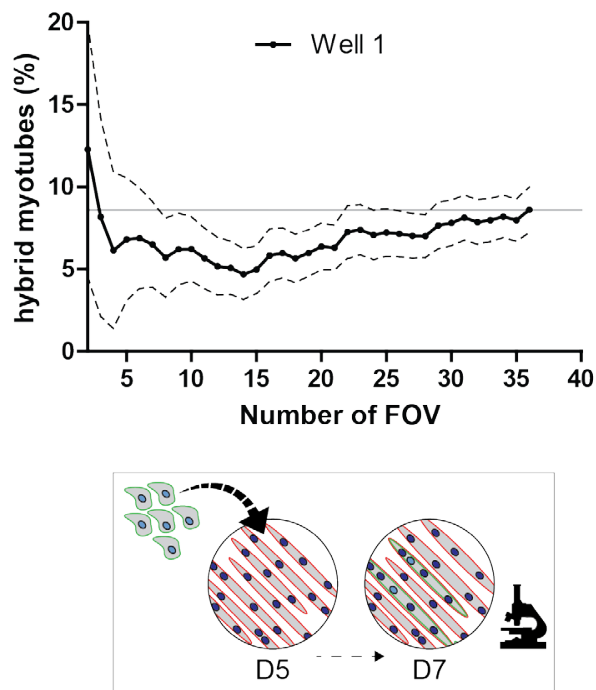
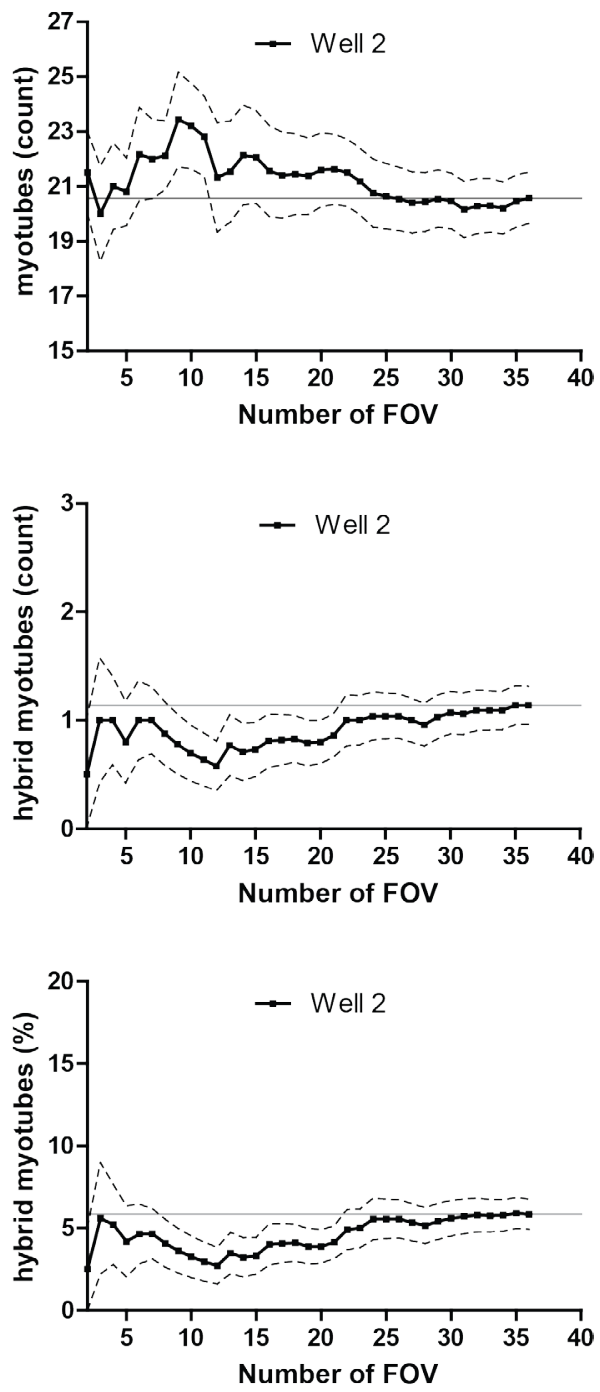

mean

SD

Figure S3. | Moving average and variation per well by increasing number of fields of view (FOV). A) moving average of total number of myotubes per well in well 1 (left) and well 2 (right), B) moving average of number of hybrid myotubes per well in well 1 (left) and well 2 (right), C) moving average of \% hybrid myotubes per well in well 1 (left) and well 2 (right). 


\section{CHAPTER 6}
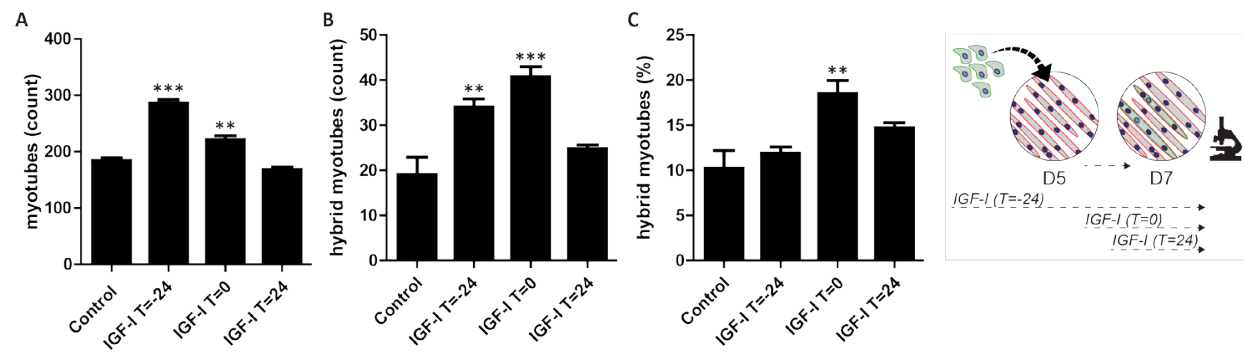

Figure S4. | Staining-based assessment of myonuclear accretion 2 days after initiation of co-culturing $+/-10 \mathrm{nM}$ IGF-I treatment started 24 hours before start of co-culturing $(T=-24)$, upon co-culturing $(T=0)$, or 24 hour after start of co-culturing $(T=24)(A-C)$. A) total number of myotubes, $B)$ number of hybrid myotubes, C) \% hybrid myotubes. Values are means $\pm S E M, n=4 .{ }^{*} p<0.05,{ }^{* *} p<0.01$, ${ }^{* * *} p<0.001$ compared to control. 
A

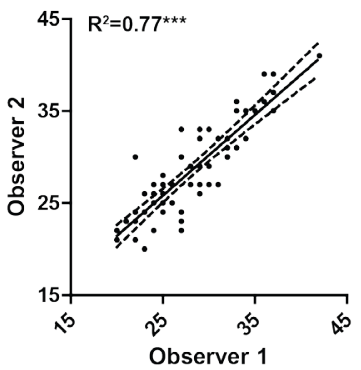

C

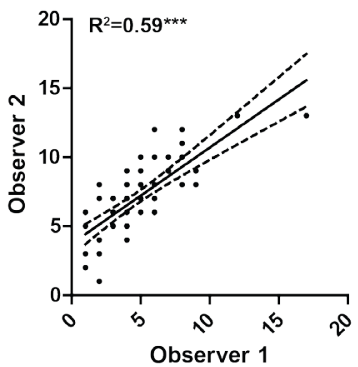

E

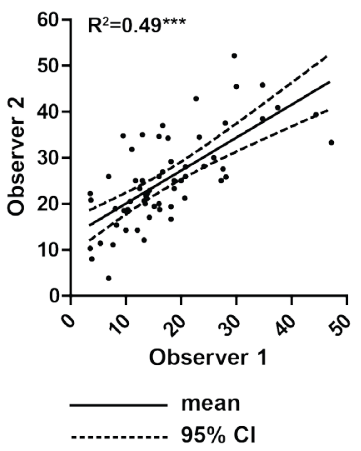

B

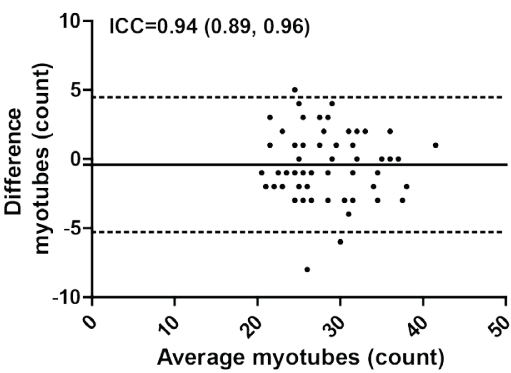

D

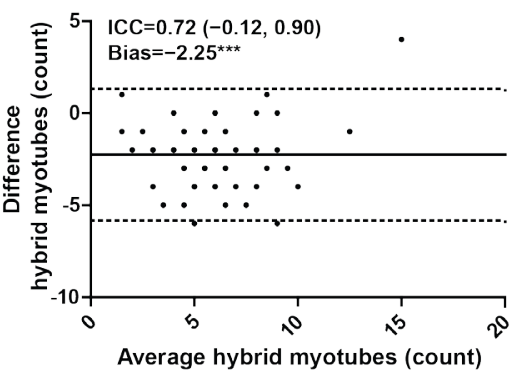

$\mathbf{F}$

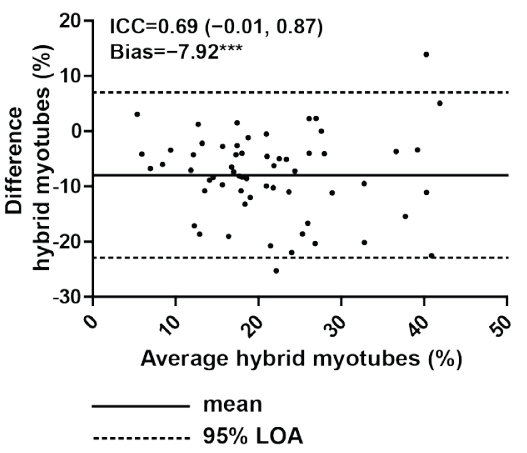

Figure S5. | Inter-rater reliability of staining-based assessment of postnatal myonuclear accretion. A) scatter plot of total number of myotubes per well by observer 1 and observer 2, B) Bland-Altman plot of total number of myotubes per well, C) scatter plot of number of hybrid myotubes per well by observer 1 and observer 2, D) Bland-Altman plot of number of hybrid myotubes per well, E) scatter plot of \% hybrid myotubes per well by observer 1 and observer 2, F) Bland-Altman plot of \% hybrid myotubes per well. Means (solid line), and the 95\% confidence interval (95\% Cl) and 95\% limits of agreement (95\% LOA) (dotted line) are displayed. Inter-rater correlation was tested by Pearson correlation (R2), absolute inter-rater agreement was assessed by two-way mixed model intraclass correlation coefficient (ICC) with $95 \% \mathrm{Cl}$, and fixed bias was assessed by Bland-Altman analysis and tested by one sample t-test. ${ }^{* * *} p<0.001$. 


\section{CHAPTER 6}

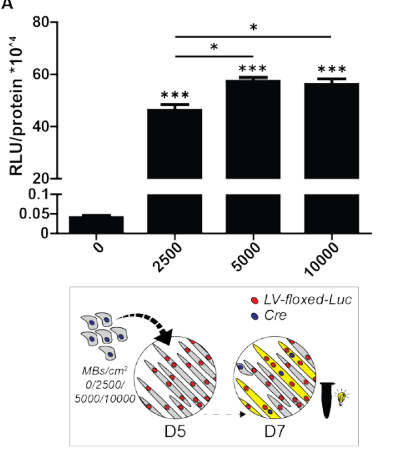

B

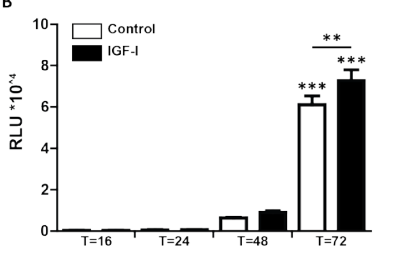

C

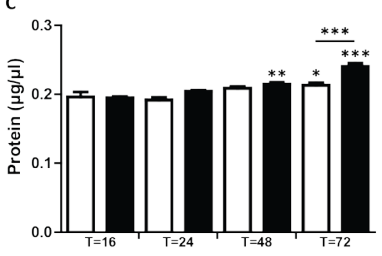

D
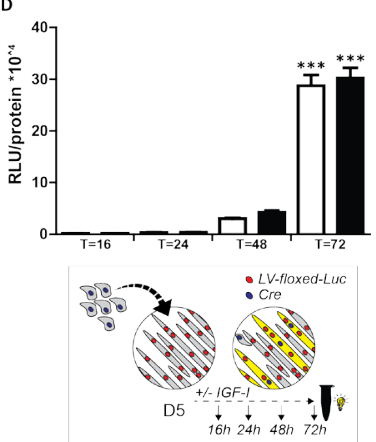

E

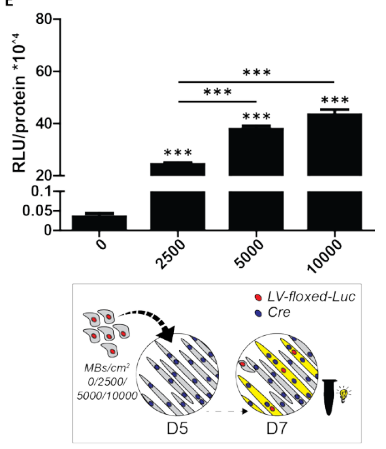

$\mathrm{F}$

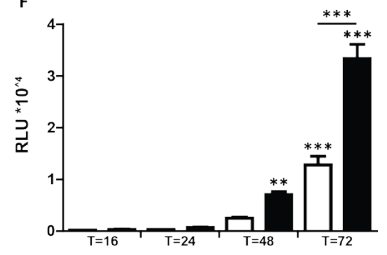

G

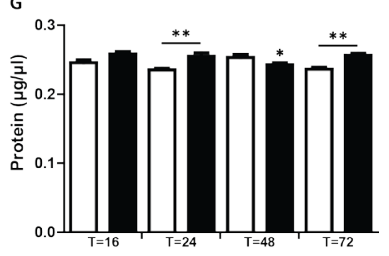

$\mathrm{H}$

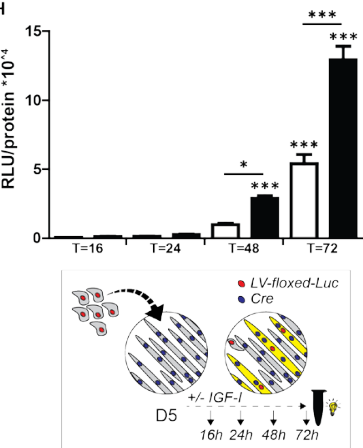

Figure S6. | Optimization of Cre/LoxP-based assessment of myonuclear accretion. Co-culture of LVfloxed-LuC C2C12 myotubes with Cre C2C12 myoblasts (A-D). A) relative luciferase activity (RLU/ protein content) per well 2 days after addition of 0,2500, 5000 or 10000 myoblasts $/ \mathrm{cm}^{2}(n=3)$. Cells lysed at indicated time points (hours) after initiation of co-culturing +/- $10 \mathrm{nM} \mathrm{IGF-I} \mathrm{(n=8),} \mathrm{B)}$ luciferase activity ( $R L U)$ per well, C) protein content ( $\mu \mathrm{g} / \mu \mathrm{L})$ per well, D) relative luciferase activity (RLU/protein content) per well. Co-culture of Cre C2C12 myotubes with LV-floxed-LuC C2C12 myoblasts $(E-H)$. E) relative luciferase activity (RLU/protein content) per well 2 days after addition of $0,2500,5000$ or 10000 myoblasts $/ \mathrm{cm}^{2}(n=3)$. Cells lysed at indicated time points (hours) after initiation of co-culturing +/- $10 \mathrm{nM} \mathrm{IGF-I} \mathrm{(} n=8)$, F) luciferase activity (RLU) per well, G) protein content $(\mu \mathrm{g} / \mu \mathrm{L})$ per well, $H$ ) relative luciferase activity (RLU/protein content) per well. Values are means $\pm S E M$. ${ }^{*} p<0.05,{ }^{* *} p<0.01,{ }^{* * *} p<0.001$ compared to 0 cells $/ \mathrm{cm}^{2}$ or compared to $T=16$ within each condition, and between indicated bars. 

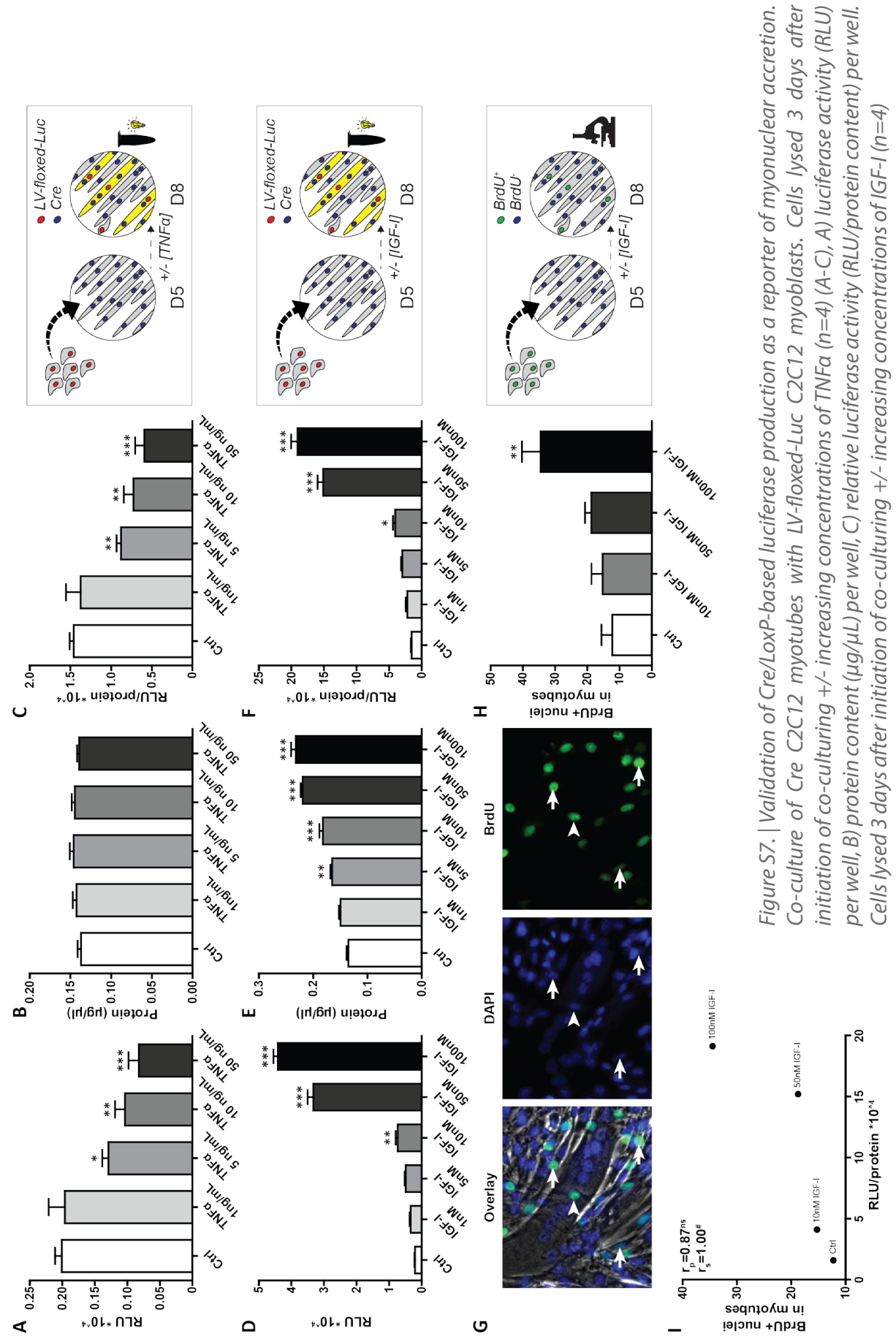


\section{CHAPTER 6}

$(D-F), D)$ luciferase activity $(R L U)$ per well, E) protein content $(\mu g / \mu L)$ per well, F) relative luciferase activity (RLU/protein content) per well. G) myonuclear accretion of $\mathrm{BrdU}^{+}$myoblast nuclei into myotubes, 3 days after initiation of co-culturing (DAPI/nuclei: blue; BrdU: green). Arrows indicate examples of $\mathrm{BrdU}^{+}$myoblast nuclei, arrow head indicates a BrdU+ nucleus in a myotube. H) Average number of BrdU $U^{+}$nuclei in myotubes per well 3 days after initiation of co-culturing +/- 10 nM IGF-I, $50 \mathrm{nM}$ IGF-I, or $100 \mathrm{nM}$ IGF-I. I) scatter plot of the relative luciferase signal (RLU/protein content) and $\mathrm{BrdU}^{+}$nuclei in myotubes, 3 days after initiation of co-culturing +/- $10 \mathrm{nM}$ IGF-I, $50 \mathrm{nM} \mathrm{IGF-I,} \mathrm{or} 100 \mathrm{nM}$ IGF-I. Abbreviations: $r P$, Pearson correlation coefficient; $r$, Spearman correlation coefficient. Values are means \pm SEM. ${ }^{*} p<0.05,{ }^{* *} p<0.01,{ }^{* * *} p<0.001$ compared to control; \#p<0.1 for correlations. 
In vitro model of postnatal myonuclear accretion
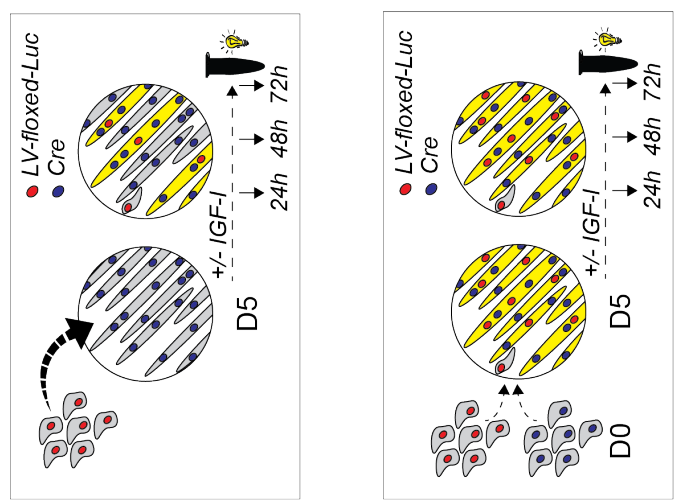

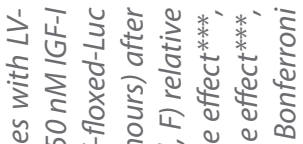

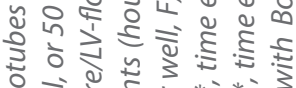
हิ心 ฟ ปิ

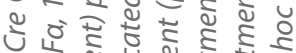

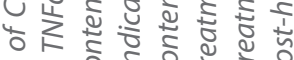
०

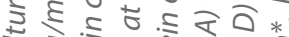
ปิ

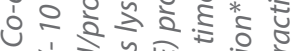
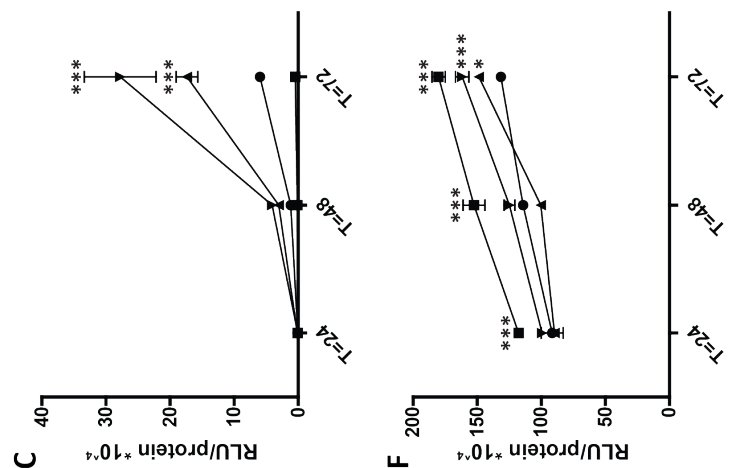

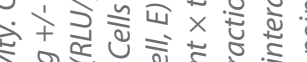

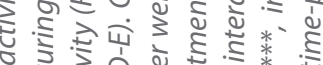

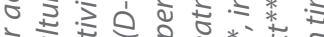

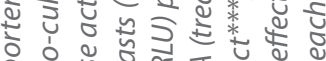
ฆे व व तิ

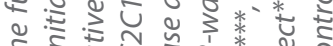

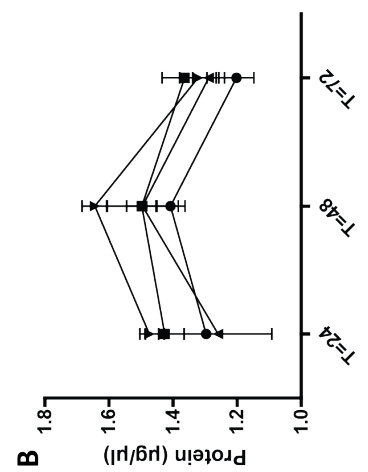

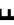

$\tau$

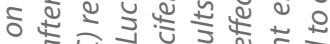

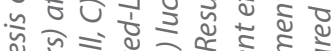

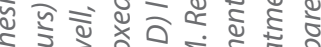

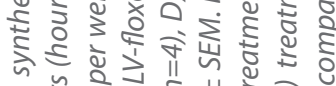

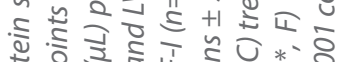
ปั ¿े ₹

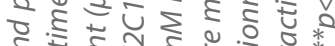
ธ

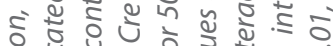

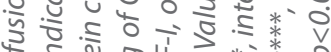

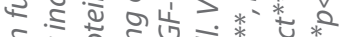

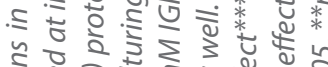

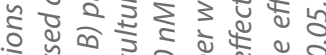

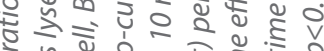
ํํำ

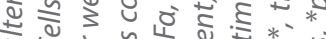

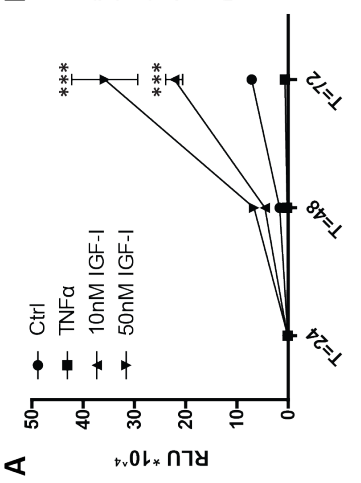

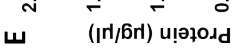

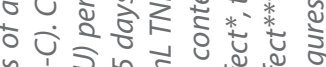
च ए वे चे के के ले वे

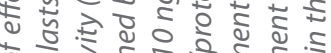
० $\frac{0}{0}$ :

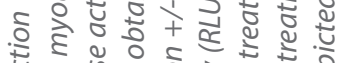

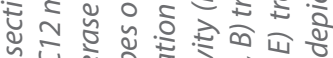

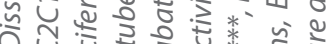

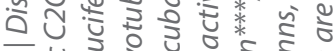

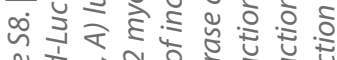

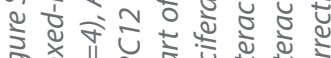

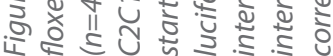



PRELIMINARY REPORT: INCREASESD MYONUCLEAR ACCRETION DURING RECOVERY FROM METABOLICSTRESS

ANITA E.M. KNEPPERS LEX B. VERDIJK MARCO C.J.M. KELDERS MITJA LAINSCAK LUC J.C. VAN LOON ANNEMIE M.W.J. SCHOLS \& RAMON C.J. LANGEN 
GENERAL DISCUSSION 


\section{CHAPTER 8}

The progressive loss of skeletal muscle mass and function is frequently observed in elderly and patients with chronic diseases such as COPD. This may lead to sarcopenia, which affects physical functioning and metabolic health. The prevention or treatment of sarcopenia requires a better understanding of the underlying cellular and molecular pathobiology. Although skeletal muscle catabolic processes are broadly studied, a potential role for impaired skeletal muscle recovery in the development of sarcopenia is still underexplored. In this thesis, we studied mechanisms involved in skeletal muscle recovery, and assessed the implications of potential disturbances in these processes for skeletal muscle maintenance in COPD patients. In this final chapter we will discuss the findings in a broader perspective and address the implications. Finally, we define important goals for future research in this area.

\section{Temporal regulation of remodeling-related processes during recovery from skeletal muscle atrophy}

It is generally accepted that the loss of muscle mass results from a change in the dynamic protein turnover balance leading to a net catabolic state, as nicely illustrated by Atherton et al. [1]. The relative contribution of alterations in rates of muscle protein synthesis (MPS) and muscle protein breakdown (MPB) to disuse-induced atrophy is highly relevant for the development of targeted interventions, but is still subject to debate. Numerous studies have shown a decline in basal and post-prandial MPS upon disuse [2-5]. However, due to a lack of data from studies in humans, disuse-induced changes in MPB are less clear-cut [6]. Similarly, alterations in MPB upon remobilization after disuse-induced atrophy remain underexplored in humans. Although a compensatory decrease in MPB upon remobilization may be expected, a temporary increase in MPB was shown after exercise [7-9], and may also be anticipated upon remobilization. Furthermore, postprandial MPS normalizes or even increases upon remobilization after disuse-induced atrophy $[7,8,10,11]$.

Interestingly, but unsurprisingly, several studies showed a lack of association between exercise-induced changes in MPS and the eventual change in muscle mass [12-14]. This could be partially due to the relatively short-term duration of protein turnover measurement, which may incompletely reflect the cumulative effects of dynamic temporal changes in MPS and MPB rates [15]. Alternatively, this lack of correlation could be due to an underappreciated role for MPB in the determination of muscle mass [15]. A further complicating factor in the interpretation of increases in MPS may be a differential temporal regulation of MPS depending on the mode of induction. There is strong evidence that, in addition to resistance exercise, endurance exercise also triggers the induction of MPS [16-20], while this is generally not accompanied 
by a substantial gain in muscle mass. Based on this discrepancy, a potential role for increased MPS in muscle reconditioning and remodeling has been extensively discussed [14, 21, 22]. In line with this notion, a more recent study showed that in the initial exercise response, MPS tends to correlate with Z-band streaming as a marker of muscle damage, but not with muscle fiber cross-sectional area (CSA) [23]. The authors of that study speculated that the increase in MPS in this context is more related to the regulation of muscle repair than of muscle mass [23, 24]. Together, these studies clearly indicate that, in addition to a role in the regulation of muscle mass, alterations in MPS are also essential in the regulation of muscle quality.

Technical limitations in the measurement of MPB prevent the further dissection of the temporal protein turnover dynamics, which is imperative for a comprehensive understanding of its role in the regulation of muscle mass and quality, particularly in the context of skeletal muscle recovery from atrophy. However, repeated assessment of the molecular signals involved in the control of muscle protein turnover may provide further insight in the temporal alterations in MPS and MPB by achieving a higher timeresolution. Moreover, molecular signals control both the changes in muscle mass and muscle quality, and as such, likely best reflect the coordination between these processes.

Hindlimb-suspension and reloading in rodents is a well-established model for disuse atrophy and recovery that can facilitate high-resolution assessment of the dynamics of skeletal muscle molecular signals involved in muscle plasticity in these conditions. By studying an elaborate panel of molecular markers in this mouse model, it was shown that acutely upon reloading, both protein synthesis and protein degradation signaling are increased [25] (Chapter 4) (Figure 1). Although UPS signaling normalized or even decreases after longer-term reloading, the increase in autophagy signaling was sustained up to the later phases of reloading [25] (Chapter 4). This is in line with the recently implicated roles for autophagy in the remodeling of skeletal muscle cellular components [9, 26-29], e.g. by promoting the removal, recycling, and replacement of damaged and dysfunctional components [28]. Autophagy not only seems to play an important role in muscle fiber remodeling, but also in the metabolic remodeling of satellite cells required for their functional maintenance [30]. In line with the parallel activation of autophagy and myogenesis reported in vitro [31], we observed a concomitant induction of myogenesis and autophagy in vivo during recovery from skeletal muscle atrophy in mice [25] (Chapter 4) (Figure 1). Upon remobilization, we previously showed the sequential induction of proliferation and differentiation markers [25], and now extend these findings by showing the 'late phase' induction 


\section{CHAPTER 8}

of a novel marker of satellite cell fusion, i.e., Myomaker (Chapter 4). The resulting functional contribution of satellite cells to the myonuclear pool is implied by the 'late phase' recovery of the myonuclear content (Chapter 4), thereby promoting myofiber maintenance and remodeling via protein synthesis with or without hypertrophy [32]. In parallel with the progression of myogenesis, metabolic remodeling is activated, as is evident from the induction of mitochondrial biogenesis and mitochondrial dynamics regulation, together with the recovery of OXPHOS during remobilization [33] (Figure 1). The regulation of these metabolic alterations seems to occur in a biphasic pattern, which may reflect an asynchronous response of the two different cell types involved in myonuclear accretion. We speculate that an initial induction of mitochondrial biogenesis, evidenced by elevated transcript levels of biogenesis regulatory genes, occurs in the myofiber and is facilitated by the increase in muscle protein synthesis signaling, whereas the second bout of mitochondrial biogenesis signaling originates from the differentiating satellite cells. Alternatively, completed incorporation of satellite cell-derived nuclei may contribute to a 'later phase' mitochondrial biogenesis originating from the muscle fiber, e.g. by delivery of nuclear DNA (nDNA) and mitochondrial DNA (mtDNA) to support the transcription of mitochondrial proteins.

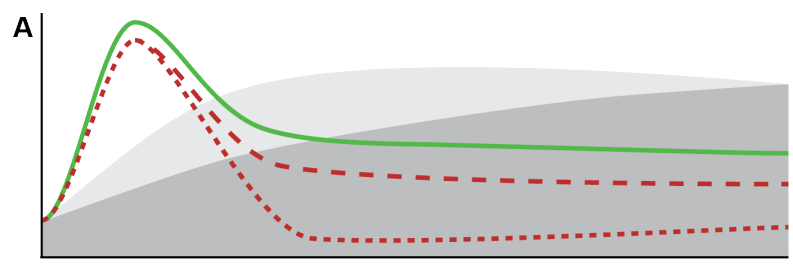

B
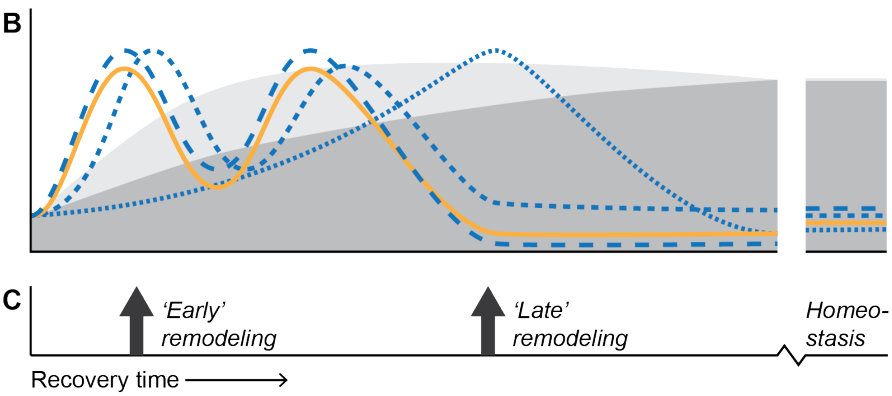

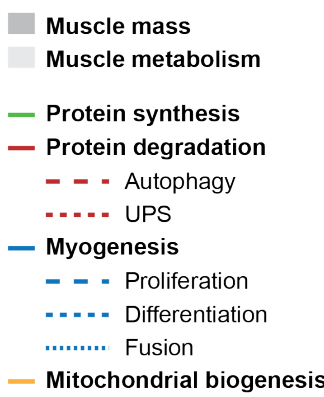

- Mitochondrial biogenesis

Figure 1. | Temporal regulation of remodeling-related processes during recovery from skeletal muscle atrophy. A. Schematic presentation of the temporal protein turnover signaling associated with the recovery of muscle mass and metabolism (as described in Chapter 4). B. Schematic presentation of the temporal regulation of myogenesis and mitochondrial biogenesis signaling associated with the recovery of muscle mass and metabolism (as described in Chapter 4). C. Hypothesized distinction between patient clusters based on the pattern of molecular and functional alterations resembling an 'early' (Cluster 2) versus 'late' stage (Cluster 1) of muscle remodeling (as described in Chapter 5). 
The temporal interrelationship between muscle mass and metabolic plasticity is evident from our work, as well as others' (Chapter 4) [26-31, 34-40]. However, applying a similar high time resolution approach as was done in the mouse study is technically and ethically very challenging in humans, preventing the direct assessment of the temporal nature of these interrelationships in vivo in humans. We took an important step towards a better understanding of these interrelationships in humans by adopting an integrative approach. By performing a cluster analysis of the correlations between remobilization-induced changes in molecular signaling after limb immobilization, we found a tight coordination specifically between the regulation of autophagy and myogenesis, and, more generally, between regulators of muscle mass and metabolic plasticity (Chapter 4).

Due to the cross-sectional nature and technical limitations of most studies in humans, it remains difficult to establish the role for these coordinated responses in the longterm regulation of muscle mass versus muscle remodeling. However, a recent study prospectively assessed the satellite cell response to exercise depending on the training status, and found an acute exercise-induced increase in satellite cell numbers in the untrained state, and a sustained elevation with no further exercise-induced alterations in satellite cell numbers in the later weeks of a training program [24]. In line with the time-point dependent correlations with exercise-induced muscle damage and alterations in MPS and MPB, the authors speculated that the initial increase in satellite cell numbers supports skeletal muscle repair, whereas the sustained elevation supports skeletal muscle hypertrophy [23, 24], as may also be the case during remobilization-induced recovery from atrophy. As such, the regulation of skeletal muscle mass and metabolic plasticity depends on the training status. This may not solely reflect training-induced myocellular intrinsic changes, but also adaptations in the myocellular microenvironment (e.g. neuromuscular junctions (NMJ), vasculature, and extracellular matrix (ECM)) [41-45]. In line, disuse induces NMJ degeneration [46], capillary loss [47, 48], and ECM remodeling [45, 49], which are reversed upon remobilization and exercise training $[45,50-56]$. Adding to the complexity of skeletal muscle recovery responses, not only these local factors, but also systemic factors are important mediators of remobilization-induced skeletal muscle recovery. For example, during skeletal muscle recovery from disuse or injury, macrophage invasion contributes to the removal of damaged cells, and contributes to muscle repair, growth and regeneration $[57,58]$. Although these factors were not subject of investigation in the current thesis, they may be important contributors to the inter-individual variability in molecular exercise responses, and as such, should be subject of future investigation when studying muscle recovery. 


\section{CHAPTER 8}

\section{Indications for altered skeletal muscle remodeling-related processes in COPD}

Skeletal muscle disuse is an important determinant of muscle wasting in patients with COPD [59]. However, in addition to the structural and metabolic alterations driven by a low amount of physical activity, COPD patients display an exaggerated impairment in oxidative phenotype compared to activity-matched healthy subjects [60]. Moreover, COPD patients with a low muscle mass seem to display an exacerbated impairment in oxidative phenotype [61-63]. As such, it was previously suggested that the impaired oxidative phenotype may accelerate the loss of muscle mass [64]. However, despite the fact that the overlap between regulatory mechanisms of muscle mass and quality implies their association, a causal role for potential disturbances of this relationship in the development of COPD-induced skeletal muscle pathology remains to be established.

Primarily based on animal studies, it could be hypothesized that COPD disease-related drivers of muscle qualitative alterations, such as hypoxia and systemic inflammation, mainly contribute to muscle wasting by the excessive induction of protein degradation [65]. Interestingly, extensive review of the fragmented human tissue data in the existing literature suggested not only an induction of catabolic processes, but also of anabolic processes in the skeletal muscle of patients with COPD [59] (Chapter 2). To obtain a better understanding of changes in shared muscle mass and remodeling-related mechanisms, we studied the regulation of muscle protein synthesis and degradation in COPD patients with and without sarcopenia, and in healthy controls. In addition, we studied the molecular regulation of the anabolic process 'myogenesis'. Through a comprehensive analysis of both anabolic and catabolic signaling, we confirmed the concomitant induction of both protein degradation, and protein synthesis pathways in patients with COPD (Chapter 3). Strengthened by the accompanying induction of myogenesis (Chapter 3), we suggest that these alterations may reflect ongoing repair or remodeling of the skeletal muscle. While COPD disease-related factors per se may drive remodeling processes $[66,67]$, there are also indications for elevated levels of damage in the skeletal muscle of COPD patients. For example, sarcomere disruption [68], and oxidative damage to muscle proteins [68, 69] and mtDNA [70] are increased in the skeletal muscle of COPD patients. Interestingly, the effects of such induction of skeletal muscle damage may be exacerbated by the COPD-associated fiber type shift, as type II fibers are more susceptible to inflammation and oxidative stress-induced damage [71-73]. As such, the fiber type shift may contribute to muscle wasting. Indeed, in line with a more pronounced fiber type shift in patients with a low muscle mass [74], there are indications for increased (oxidative) muscle damage in these patients $[68,74]$. Moreover, we found that the COPD-related increase in protein turnover 
signaling was most prominent in sarcopenic patients (Chapter 3), suggesting even more pronounced remodeling in skeletal muscle of these patients. In contrast, the induction of myogenesis signaling was not further enhanced in sarcopenic patients (Chapter 3). Contrary to aging [75], there is no clear evidence for a further depletion of the satellite cell pool in skeletal muscle of COPD patients [76, 77]. Nevertheless, in line with the 'inadequate' increase in myogenesis signaling, muscle wasted COPD patients display replicative exhaustion, and potentially resulting senescence of satellite cells [77-79]. Unfortunately, we were not able to directly measure satellite cell numbers or function in the COPD patient cohort studied in this thesis (Chapter 3 \& Chapter 5). However, given the crucial role of myogenesis in muscle repair and regeneration [80-86], we propose that impaired myogenesis, evidenced by an inadequate adaptive increase in myogenesis signaling, may result in impaired muscle quality maintenance and thereby contribute to the progressive decline of muscle mass in COPD patients.

An inadequate increase in myogenic signaling may reflect an exhaustion of the systems regulating skeletal muscle mass and metabolic plasticity. As such, COPDassociated baseline alterations may prevent the induction of these signaling pathways involved in the skeletal muscle recovery from atrophy, and the response to exercise training as deployed during pulmonary rehabilitation programs. However, several studies assessed molecular markers of remodeling-related processes upon exercise training in COPD patients [87-91], and did not find a clear differential rehabilitation response between predefined phenotypic categories. Similarly, we did not find a clear differential molecular rehabilitation response pattern between the COPD patients with or without sarcopenia studied in Chapter 3 [unpublished data]. Moreover, the rehabilitation-induced change in 6MWT and peak load on a cycle ergometer did not differ between these sarcopenic and non-sarcopenic patients [unpublished data]. Interestingly, only 3 patients (7\%) displayed no change or a decrease in both the $6 \mathrm{MWT}$ and peak load. This suggests that in line with findings in healthy subjects [92, 93], nearly all COPD patients functionally improve after supervised exercise training. Nevertheless, as generally accepted, there is a large inter-individual variation in the response to exercise or rehabilitation. In part, this may explain the lack of clear differences in the molecular rehabilitation response between predefined phenotypic categories (e.g. based on muscle mass). However, we should also consider that the skeletal muscle molecular changes that drive the loss of muscle mass and quality, may not necessarily occur in parallel with the development of phenotypic alterations. To identify divergent molecular patterns that may be underlying to the development of skeletal muscle pathology, we performed an unbiased analysis of the rehabilitationinduced molecular alterations in skeletal muscle of COPD patients. Thereby, we 


\section{CHAPTER 8}

identified two major patient clusters, which were distinguished by a pattern of molecular and functional alterations resembling an 'early' versus 'late' stage of muscle remodeling (Chapter 5) (Figure 1).

The separation of patients in these two response-patterns may have several causes, likely reflecting either an 'impaired', or 'delayed/blunted' remodeling response in the 'early' stage remodeling group. Several studies found indications for an altered progression of myogenesis in the isolated muscle satellite cells from skeletal muscle of COPD patients [77, 94], which may be linked to a defective metabolic plasticity [31]. Such an altered remodeling response would suggest that, even upon long-term exercise training, skeletal muscle adaptation would remain incomplete. Although an impaired remodeling response may originate from a failed induction of remodelingrelated signaling, a failed progression of these processes seems more plausible given the pattern of 'early' response, suggesting that the initial induction of the process is still present. However, while an impaired response would suggest that the short-term hypertrophic effects of exercise training may not be maintained over time [86], several studies have shown that long-term exercise training effectively induces health benefits in COPD patients [95-100]. Moreover, there is evidence for exercise-induced metabolic and morphological adaptations in the skeletal muscle of these patients [101, 102]. These data suggest a delayed or blunted remodeling response rather than an impaired response in COPD patients. As in healthy subjects, the speed and amplitude of the remodeling-response depends on the initial training status, reflecting myocellular 'intrinsic' and microenvironmental 'extrinsic' characteristics. Indeed, it was shown in healthy subjects that the extent of muscle fiber capillarization and innervation are associated with the exercise-induced satellite cell response and muscle recovery following disuse $[103,104]$. In line with a potentially delayed or blunted remodeling response to exercise in a subgroup of COPD patients, there is evidence for a decreased capillarization, and persistent denervation of skeletal muscle in COPD patients [61, 105-108]. Nonetheless, these parameters can still be improved through exercise training [53, 109]. In addition to these microenvironmental characteristics, COPDrelated systemic factors may contribute to a delayed or blunted remodeling response to exercise. Interestingly, it was indeed shown that systemic inflammation in COPD is associated with an increased macrophage number and abnormal polarization [110, 111], which may delay the progression of myogenesis [112-114]. Again, this supports our hypothesis that myogenesis may be delayed or blunted rather than impaired in a subgroup of COPD patients. 


\section{A central role for satellite cells in muscle fiber remodeling}

Given the clear indications for a tight interrelationship between the cell's structure and molecular systems that regulate skeletal muscle mass and muscle quality, it is essential to identify central regulators that may serve as a target for intervention. Evidently, protein synthesis is essential for the production of structural and functional components to support e.g. muscle fiber hypertrophy, recovery from injury, and metabolic plasticity. In turn, muscle fiber protein synthesis is controlled by myonuclei, which, according to the 'myonuclear domain' theory, each control the mRNA transcription and protein synthesis of a finite volume of cytoplasm (i.e., myonuclear domain) $[115,116]$. As such, extensive and sustained myofiber expansion would require myonuclear accretion. In addition, the myonuclear domain size does not just depend on the synthetic capacity, but also depends on the rate of protein degradation. This would suggest that conditions that require an increased protein synthesis in the absence of muscle growth, such as muscle repair and remodeling, may similarly require myonuclear accretion (Figure 2). Indeed, myonuclear accretion is facilitated by satellite cells, which were shown to be indispensable for both muscle hypertrophy and repair [80-86].

Interestingly, a non-hypertrophic increase in protein synthesis is also necessary for muscle qualitative remodeling. In line, it has become clear that satellite cells contribute to the muscle aerobic metabolic adaptation, as recently reviewed by Abreu et al. [34]. Specifically, in this context, it should be noted that not all myocellular proteins are encoded by the $\mathrm{nDNA}$, but several metabolic genes are encoded by the $m t D N A$. Although in contrast to nDNA, mtDNA can be replicated in the post-mitotic muscle fibers, excessive DNA damage may prevent this [117]. Importantly, mtDNA is more sensitive than $\mathrm{nDNA}$ to oxidative stress [118], which is a well-known driver of muscle pathology in COPD. Furthermore, impaired mtDNA quality has been linked to a decrease in oxidative phenotype, a change in fiber type, and sarcopenia [119121], which are all characteristic of COPD-induced skeletal muscle pathology. Previous reports suggest that satellite cells support the restoration of mtDNA quality [122, 123], which may potentially occur via the direct donation of satellite cell mtDNA to the muscle fiber [124, 125], and may be particularly important for muscle maintenance in COPD patients. 


\section{CHAPTER 8}

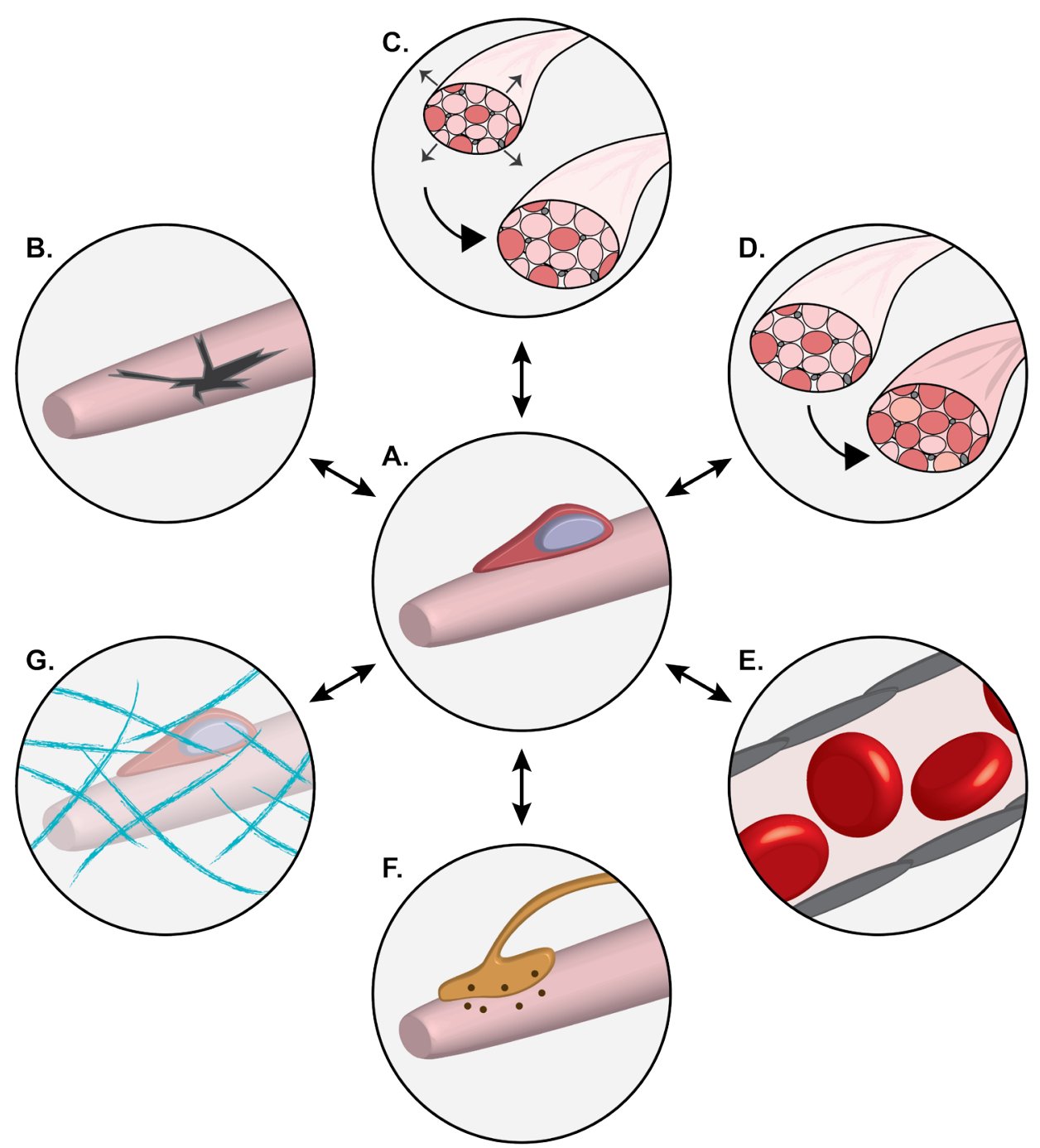

Figure 2. | A central role for satellite cells in the plasticity of muscle mass and muscle quality. A. Satellite cell-derived myonuclear accretion. B. Muscle fiber structural and molecular damage. C. Muscle fiber hypertrophy. D. Muscle fiber phenotypic and metabolic alterations. E. Muscle vasculature. F. Neuromuscular junctions. G. Extracellular matrix.

\section{Satellite cell cross-talk}

Evidently, a plethora of physiological conditions can lead to the induction of myonuclear accretion, suggesting that satellite cells 'sense' a need for myonuclear accretion. This implies a well-controlled cross-talk between the muscle fiber and satellite cell, in order to control satellite cell activation, its number of replicative cycles, the timely induction of myogenic differentiation, and the guidance of fusion. However, 
cross-talk between muscle fibers and satellite cells is difficult to study in vivo, and in vitro models are not equipped to study the regulation of myoblast-myotube fusion as occurs during postnatal myogenesis. Therefore, we developed an in vitro model that better reflects the aspects of postnatal myogenesis, and that facilitates the study of myotube-myoblast cross-talk (Chapter 6).

In the context of skeletal muscle growth and regeneration, the extrinsic regulation of satellite cell function has been extensively reviewed [126, 127]. Since several physiological conditions that evoke adaptations in the myofibers can lead to the induction of myonuclear accretion, factors that induce satellite cell activation and differentiation likely originate from the muscle fiber. Indeed, when in vitro myotubes were subjected to an atrophic trigger, subsequent myonuclear accretion was increased during myotube recovery (Chapter $6 \&$ Chapter 7). Through the use of myotube conditioned medium, we revealed the involvement of a secreted factor in this response (Chapter 7). Myotubes and myofibers have been reported to secrete myokines, and extracellular vesicles containing microRNA's, which have a role in the regulation of satellite cell function, as extensively reviewed [128-131]. Interestingly, myokine secretion has not only been reported in the context of muscle mass regulation, but also in the regulation of metabolism [132]. In line, we found that disruption of myotube metabolism induces an increase in myonuclear accretion during recovery (Chapter 7). In accordance with previous reports [133, 134], myotube metabolic disruption was shown to induce the expression of FGF21 (Chapter 7). FGF21 is an interesting candidate regulator of myonuclear accretion to control muscle metabolic alterations, as FGF21 has been associated with the progression of myogenesis and the formation of, specifically, aerobic fibers [135-137]. A general role of FGF's has been shown in satellite cell activation, differentiation, and self-renewal, but the effect on fusion had not been studied yet [138]. We showed for the first time that myotube FGF21 knockdown led to a decrease in in vitro postnatal myonuclear accretion (Chapter 7), implicating FGF21 as a myotube-derived regulator of myotube-myoblast cross-talk during the regulation of muscle plasticity. This notion is supported by the expression of FGF receptors in satellite cells [139].

Considering the close proximity of satellite cells to myofibers, a direct interaction between these cell types through membrane bound proteins is anticipated. Indeed, as reviewed, an extensive interaction of membrane receptors is involved in the regulation of satellite cell fusion during muscle development [140]. To date, no distinction is made between developmental and postnatal regulation of satellite cell fusion, but the role of several membrane-bound proteins, such as Myomaker and Myomixer, in the 


\section{CHAPTER 8}

regulation of adult skeletal muscle maintenance is verified [141]. Importantly, using our in vitro model, we were able to show that Myomaker knockdown, specifically in myoblasts, impairs postnatal myonuclear accretion (Chapter 6).

Membrane bound proteins and myokines not only modulate the cross-talk between the muscle-fiber and satellite cell, but also control the communication with other structures in the skeletal muscle, as well as the communication between skeletal muscle and other organs [41, 142]. Interestingly, satellite cells display a direct interaction with a structural part of the niche, i.e., the ECM, which modulates satellite cell function [143, 144] (Figure 2). Conversely, satellite cells regulate ECM deposition [86], which may be mediated by fibroblasts [83, 145-147]. In addition, excessive fibrosis and failed regeneration were shown upon inhibition of macrophage functioning [148]. This likely reflects the important role for macrophages in the regulation of satellite cell function $[57,58]$. Interestingly, secreted factors from the muscle fiber regulate macrophage activation, and potentially macrophage polarization [149]. As such, macrophages may play a mediating role in muscle fiber - satellite cell cross-talk, even in the absence of muscle trauma. Recent evidence also implied a mediating role for macrophages in the coupling between myogenesis and angiogenesis [150, 151]. In turn, the level of muscle fiber capillarization is associated with the exercise-induced satellite cell response [53, 103], which potentially results from a direct interaction between endothelial cells and satellite cells $[152,153]$ (Figure 2). Additionally, a regulating role for satellite cells in the maintenance and regeneration of the NMJ was proposed [154, 155], whereas denervation is clearly associated with satellite cell loss [156, 157] (Figure 2). Together, this shows that cross-talk within and between different cell types in the skeletal muscle is important for the satellite-cell mediated regulation of muscle mass and quality, and disturbances in satellite cell function may contribute to aberrations of multiple muscle tissue-associated structures in COPD.

\section{Therapeutic perspectives and future research in COPD}

The studies described in this thesis, and recent advances in the literature clearly indicate that postnatal myogenesis plays a central role in the regulation of muscle mass and metabolic plasticity. Moreover, the observed COPD-related disturbances in this process seem to be underlying to both the loss of skeletal muscle mass and quality in COPD patients. Considering the clear interrelationship between the regulation of muscle mass and quality, treatments that target the observed skeletal muscle alterations may result in severe off-target effects. For example, in mice, inhibition of protein degradation leads to an impaired recovery from skeletal muscle atrophy [158]. Therefore, it is imperative to identify the central regulators, and underlying drivers of 
COPD-associated skeletal muscle mass and quality loss.

Based on the studies presented in this thesis, and the recent literature, we propose a central role for satellite cell dysfunction in the development of COPD-related muscle pathology. Interestingly, the replicative exhaustion underlying satellite cell dysfunction in COPD patients is also a hallmark of aging [159]. Thus, depending on the employed definition, COPD-associated satellite cell dysfunction and disturbed myogenesis may at least partially be considered as the results of accelerated aging. Satellite cell dysfunction could directly contribute to both the COPD and age-associated muscle pathology, by preventing the turnover of myonuclei and myofiber components, and by obscuring the direct interactions with the myocellular microenvironment. Notably, the characteristics of COPD- and age-associated muscle pathology differ, e.g. evidenced by a differential shift in fiber type distribution. Nevertheless, satellite cell dysfunction may also be underlying to age- or disease-specific muscle alterations, due to its crucial role in the removal and recovery from muscle fiber damage. For example, COPD is often associated with systemic inflammation and oxidative stress, which can lead to the induction of damage to the nDNA and mtDNA. The removal of this damage is partly mediated by satellite cell mediated DNA turnover, and as such, impairments in this system may lead to DNA damage accumulation and the subsequent loss of muscle mass and oxidative capacity characteristic for COPD [70, 160, 161]. Moreover, systemic inflammation and oxidative stress seem to drive the production of specific microRNA's, including miR-206 $[162,163]$, which was shown to be elevated in the circulation of COPD patients [164]. This microRNA is a paracrine signal that promotes myogenesis $[163,165]$, but a systemic elevation may also drive the excessive ECM production in COPD patients [145-147, 166].

Based on this, it seems reasonable to aim therapies for COPD patients at the suppression of systemic inflammation and oxidative stress. However, certain levels of both immune cell activity and oxidative stress are important for satellite cell functioning, and more research is required to define a favorable modulation of these factors. In line with this, future studies should further elucidate the communication between the satellite cell and myofiber, and the modulation of this communication by COPD and age-related alterations in the satellite cell niche components. Advances in this area may be accelerated by the use of our new in vitro cell culture system. Moreover, the complexity of in vivo modulation of myonuclear accretion may be even more accurately studied by expanding the in vitro cell culture systems beyond the interaction between myotubes and myoblasts, by including factors such as the ECM characteristics. 


\section{CHAPTER 8}

Given the complexity and interrelationship between the skeletal muscle molecular regulation of mass and quality, and the interaction with the satellite cell niche components, molecular targets for intervention have not yet been defined. Although beneficial effects of exercise training have been observed, longitudinal studies will be essential to determine the long-term skeletal muscle responses to exercise training in both COPD patients and elderly, to assess the role for potential satellite cell intrinsic and extrinsic defects in an 'impaired' or 'blunted' exercise training response. To combat aging- and COPD-associated satellite cell intrinsic defects, autologous stem cell transplantation seems a promising therapy $[167,168]$. For example, by exvivo expansion of the satellite cell pool, potential harmful effects of the pathological satellite cell niche on satellite cell activation and proliferation can be circumvented. Furthermore, a satellite cell pool with intrinsic defects preventing proliferation, differentiation, or fusion can be renewed by transplantation of mesenchymal stem cells (MSC). Moreover, co-transplantation of satellite cells with MSCs is of particular interest, as this further improves skeletal muscle regeneration likely due to a beneficial modification of the satellite cell niche by MSCs [169]. However, despite recent advances, this treatment option is still in its infancy, as beneficial effects in humans have yet to be proven, and fundamental challenges such as the development of appropriate delivery systems remain to be solved. Conversely, the most comprehensive and effective intervention to date remains exercise training, which benefits skeletal muscle mass and function in both COPD patients and elderly. To better understand variability in the exercise/ rehabilitation response and the association with the progressive loss of muscle mass and function, future studies should include the characterization of baseline differences, and the sequential rehabilitation-induced modification of satellite cell niche components in patients with COPD. Thereby, we may be able to select training modalities that most potently improve the factors that limit the rehabilitation response in the different identified patient clusters in this thesis. 


\section{REFERENCES}

1. Atherton PJ, Greenhaff PL, Phillips SM, et al. Control of skeletal muscle atrophy in response to disuse: clinical/preclinical contentions and fallacies of evidence. Am J Physiol Endocrinol Metab 2016;311(3):E594-604.

2. de Boer MD, Selby A, Atherton $\mathrm{P}$, et al. The temporal responses of protein synthesis, gene expression and cell signalling in human quadriceps muscle and patellar tendon to disuse. $J$ Physiol 2007;585(Pt 1):241-51.

3. Gibson JN, Halliday D, Morrison WL, et al. Decrease in human quadriceps muscle protein turnover consequent upon leg immobilization. Clin Sci (Lond) 1987;72(4):503-9.

4. Glover El, Phillips SM, Oates BR, et al. Immobilization induces anabolic resistance in human myofibrillar protein synthesis with low and high dose amino acid infusion. J Physiol 2008;586(24):6049-61.

5. Wall BT, Dirks ML, Snijders T, et al. Short-term muscle disuse lowers myofibrillar protein synthesis rates and induces anabolic resistance to protein ingestion. Am J Physiol Endocrinol Metab 2016;310(2):E137-47.

6. Wall BT, Dirks ML, van Loon LJ. Skeletal muscle atrophy during short-term disuse: implications for age-related sarcopenia. Ageing Res Rev 2013;12(4):898-906.

7. Phillips SM, Tipton KD, Aarsland A, et al. Mixed muscle protein synthesis and breakdown after resistance exercise in humans. Am J Physiol 1997;273(1 Pt 1):E99-107.

8. Biolo G, Maggi SP, Williams BD, et al. Increased rates of muscle protein turnover and amino acid transport after resistance exercise in humans. Am J Physiol 1995;268(3 Pt 1):E514-20.

9. Tipton KD, Hamilton DL, Gallagher IJ. Assessing the Role of Muscle Protein Breakdown in Response to Nutrition and Exercise in Humans. Sports Med 2018;48(Suppl 1):53-64.

10. Tanner RE, Brunker LB, Agergaard J, et al. Age-related differences in lean mass, protein synthesis and skeletal muscle markers of proteolysis after bed rest and exercise rehabilitation. J Physiol 2015;593(18):4259-73.

11. Mitchell CJ, D'Souza RF, Mitchell SM, et al. Impact of dairy protein during limb immobilization and recovery on muscle size and protein synthesis; a randomized controlled trial. Journal of applied physiology 2018;124(3):717-28.

12. Mitchell CJ, Churchward-Venne TA, Parise G, et al. Acute post-exercise myofibrillar protein synthesis is not correlated with resistance training-induced muscle hypertrophy in young men. PLoS One 2014;9(2):e89431.

13. Mayhew DL, Kim JS, Cross JM, et al. Translational signaling responses preceding resistance training-mediated myofiber hypertrophy in young and old humans. Journal of applied physiology 2009;107(5):1655-62.

14. Mitchell CJ, Churchward-Venne TA, Cameron-Smith D, et al. What is the relationship between the acute muscle protein synthesis response and changes in muscle mass? Journal of applied physiology 2015;118(4):495-97.

15. Mitchell CJ, Churchward-Venne TA, Cameron-Smith D, et al. What is the relationship between the acute muscle protein synthesis response and changes in muscle mass? Journal of applied physiology 2015;118(4):495-7.

16. Sheffield-Moore $M$, Yeckel CW, Volpi $E$, et al. Postexercise protein metabolism in older and younger men following moderate-intensity aerobic exercise. Am J Physiol Endocrinol Metab 2004;287(3):E513-22.

17. Wilkinson SB, Phillips SM, Atherton PJ, et al. Differential effects of resistance and endurance exercise in the fed state on signalling molecule phosphorylation and protein synthesis in human muscle. J Physiol 2008;586(15):3701-17.

18. Beelen M, Zorenc A, Pennings B, et al. Impact of protein coingestion on muscle protein 


\section{CHAPTER 8}

synthesis during continuous endurance type exercise. Am J Physiol Endocrinol Metab 2011;300(6):E945-54.

19. Carraro F, Stuart CA, Hartl WH, et al. Effect of exercise and recovery on muscle protein synthesis in human subjects. Am J Physiol 1990;259(4 Pt 1):E470-6.

20. Harber MP, Konopka AR, Jemiolo $B$, et al. Muscle protein synthesis and gene expression during recovery from aerobic exercise in the fasted and fed states. Am J Physiol Regul Integr Comp Physiol 2010;299(5):R1254-62.

21. Commentaries on Viewpoint: What is the relationship between acute measure of muscle protein synthesis and changes in muscle mass? Journal of applied physiology 2015;118(4):498-503.

22. McGlory C, Devries MC, Phillips SM. Skeletal muscle and resistance exercise training; the role of protein synthesis in recovery and remodeling. Journal of applied physiology 2017;122(3):541-48.

23. Damas F, Phillips SM, Libardi CA, et al. Resistance training-induced changes in integrated myofibrillar protein synthesis are related to hypertrophy only after attenuation of muscle damage. J Physiol 2016;594(18):5209-22.

24. Damas F, Libardi CA, Ugrinowitsch C, et al. Early- and later-phases satellite cell responses and myonuclear content with resistance training in young men. PLoS One 2018;13(1):e0191039.

25. Pansters NA, Schols AM, Verhees KJ, et al. Muscle-specific GSK-3beta ablation accelerates regeneration of disuse-atrophied skeletal muscle. Biochim Biophys Acta 2015;1852(3):490506.

26. Call JA, Wilson RJ, Laker RC, et al. Ulk1-mediated autophagy plays an essential role in mitochondrial remodeling and functional regeneration of skeletal muscle. Am J Physiol Cell Physiol 2017;312(6):C724-C32.

27. Ferraro E, Giammarioli AM, Chiandotto $S$, et al. Exercise-induced skeletal muscle remodeling and metabolic adaptation: redox signaling and role of autophagy. Antioxid Redox Signal 2014;21(1):154-76.

28. Kaur J, Debnath J. Autophagy at the crossroads of catabolism and anabolism. Nat Rev Mol Cell Biol 2015;16(8):461-72.

29. Nichenko AS, Southern WM, Atuan M, et al. Mitochondrial maintenance via autophagy contributes to functional skeletal muscle regeneration and remodeling. Am J Physiol Cell Physiol 2016;311(2):C190-200.

30. Boya P, Codogno P, Rodriguez-Muela N. Autophagy in stem cells: repair, remodelling and metabolic reprogramming. Development 2018;145(4)

31. Fortini P, Ferretti C, lorio E, et al. The fine tuning of metabolism, autophagy and differentiation during in vitro myogenesis. Cell Death Dis 2016;7:e2168.

32. Burd NA, De Lisio M. Skeletal Muscle Remodeling: Interconnections Between Stem Cells and Protein Turnover. Exerc Sport Sci Rev 2017;45(3):187-91.

33. Remels AH, Pansters NA, Gosker HR, et al. Activation of alternative NF-kappaB signaling during recovery of disuse-induced loss of muscle oxidative phenotype. Am J Physiol Endocrinol Metab 2014;306(6):E615-26.

34. Abreu P, Mendes SV, Ceccatto VM, et al. Satellite cell activation induced by aerobic muscle adaptation in response to endurance exercise in humans and rodents. Life Sci 2017;170:3340.

35. Sin J, Andres AM, Taylor DJ, et al. Mitophagy is required for mitochondrial biogenesis and myogenic differentiation of C2C12 myoblasts. Autophagy 2016;12(2):369-80.

36. Kim B, Kim JS, Yoon Y, et al. Inhibition of Drp1-dependent mitochondrial division impairs myogenic differentiation. Am J Physiol Regul Integr Comp Physiol 2013;305(8):R927-38.

37. Barbieri E, Battistelli M, Casadei L, et al. Morphofunctional and Biochemical Approaches 
for Studying Mitochondrial Changes during Myoblasts Differentiation. J Aging Res 2011;2011:845379.

38. Koopman R, Ly CH, Ryall JG. A metabolic link to skeletal muscle wasting and regeneration. Frontiers in physiology 2014;5:32.

39. Ceco E, Weinberg SE, Chandel NS, et al. Metabolism and Skeletal Muscle Homeostasis in Lung Disease. Am J Respir Cell Mol Biol 2017;57(1):28-34.

40. Fortini P, lorio E, Dogliotti E, et al. Coordinated Metabolic Changes and Modulation of Autophagy during Myogenesis. Frontiers in physiology 2016;7:237.

41. Yin $H$, Price F, Rudnicki MA. Satellite cells and the muscle stem cell niche. Physiol Rev 2013;93(1):23-67.

42. Snijders T, Nederveen JP, Verdijk LB, et al. Muscle fiber capillarization as determining factor on indices of insulin sensitivity in humans. Physiol Rep 2017;5(10)

43. Takagi R, Ogasawara R, Takegaki J, et al. Influence of past injurious exercise on fiber typespecific acute anabolic response to resistance exercise in skeletal muscle. Journal of applied physiology 2018;124(1):16-22.

44. Seene T, Umnova M, Kaasik P. Morphological peculiarities of neuromuscular junctions among different fiber types: Effect of exercise. Eur J Transl Myol 2017;27(3):6708.

45. Kaasik P, Riso EM, Seene T. Extracellular matrix and myofibrils during unloading and reloading of skeletal muscle. Int J Sports Med 2011;32(4):247-53.

46. Rudolf $R$, Deschenes MR, Sandri M. Neuromuscular junction degeneration in muscle wasting. Current opinion in clinical nutrition and metabolic care 2016;19(3):177-81.

47. Kaneguchi A, Ozawa J, Kawamata S, et al. Intermittent whole-body vibration attenuates a reduction in the number of the capillaries in unloaded rat skeletal muscle. BMC Musculoskelet Disord 2014;15:315.

48. Bleeker MW, De Groot PC, Rongen GA, et al. Vascular adaptation to deconditioning and the effect of an exercise countermeasure: results of the Berlin Bed Rest study. Journal of applied physiology 2005;99(4):1293-300.

49. Urso ML, Scrimgeour AG, Chen YW, et al. Analysis of human skeletal muscle after $48 \mathrm{~h}$ immobilization reveals alterations in mRNA and protein for extracellular matrix components. Journal of applied physiology 2006;101(4):1136-48.

50. Nishimune $\mathrm{H}$, Stanford JA, Mori Y. Role of exercise in maintaining the integrity of the neuromuscular junction. Muscle Nerve 2014;49(3):315-24.

51. Wilson $\mathrm{MH}$, Deschenes MR. The neuromuscular junction: anatomical features and adaptations to various forms of increased, or decreased neuromuscular activity. Int J Neurosci 2005;115(6):803-28.

52. Goetsch SC, Hawke TJ, Gallardo TD, et al. Transcriptional profiling and regulation of the extracellular matrix during muscle regeneration. Physiol Genomics 2003;14(3):261-71.

53. Joanisse S, Nederveen JP, Snijders T, et al. Skeletal Muscle Regeneration, Repair and Remodelling in Aging: The Importance of Muscle Stem Cells and Vascularization. Gerontology 2017;63(1):91-100.

54. Charifi N, Kadi F, Feasson L, et al. Enhancement of microvessel tortuosity in the vastus lateralis muscle of old men in response to endurance training. J Physiol 2004;554(Pt 2):55969.

55. Jensen L, Bangsbo J, Hellsten Y. Effect of high intensity training on capillarization and presence of angiogenic factors in human skeletal muscle. J Physiol 2004;557(Pt 2):571-82.

56. Latroche C, Gitiaux C, Chretien F, et al. Skeletal Muscle Microvasculature: A Highly Dynamic Lifeline. Physiology (Bethesda) 2015;30(6):417-27.

57. Tidball JG, Wehling-Henricks M. Macrophages promote muscle membrane repair and muscle fibre growth and regeneration during modified muscle loading in mice in vivo. $J$ 


\section{CHAPTER 8}

Physiol 2007;578(Pt 1):327-36.

58. Bencze $M$, Negroni $E$, Vallese $D$, et al. Proinflammatory macrophages enhance the regenerative capacity of human myoblasts by modifying their kinetics of proliferation and differentiation. Mol Ther 2012;20(11):2168-79.

59. Langen RC, Gosker HR, Remels AH, et al. Triggers and mechanisms of skeletal muscle wasting in chronic obstructive pulmonary disease. Int J Biochem Cell Biol 2013;45(10):224556.

60. Gifford JR, Trinity JD, Kwon OS, et al. Altered skeletal muscle mitochondrial phenotype in COPD: disease vs. disuse. Journal of applied physiology 2018;124(4):1045-53.

61. Kapchinsky S, Vuda M, Miguez K, et al. Smoke-induced neuromuscular junction degeneration precedes the fibre type shift and atrophy in COPD. J Physiol 2018

62. Zhang JQ, Long $X Y, X i e ~ Y$, et al. Relationship between PPARalpha mRNA expression and mitochondrial respiratory function and ultrastructure of the skeletal muscle of patients with COPD. Bioengineered 2017;8(6):723-31.

63. van de Bool C, Gosker HR, van den Borst B, et al. Muscle Quality is More Impaired in Sarcopenic Patients With Chronic Obstructive Pulmonary Disease. J Am Med Dir Assoc 2016;17(5):415-20.

64. Remels $\mathrm{AH}$, Gosker HR, Langen $\mathrm{RC}$, et al. The mechanisms of cachexia underlying muscle dysfunction in COPD. Journal of applied physiology 2013;114(9):1253-62.

65. Milan G, Romanello V, Pescatore $F$, et al. Regulation of autophagy and the ubiquitinproteasome system by the FoxO transcriptional network during muscle atrophy. Nat Commun 2015;6:6670.

66. de Theije CC, Langen RC, Lamers WH, et al. Distinct responses of protein turnover regulatory pathways in hypoxia- and semistarvation-induced muscle atrophy. Am J Physiol Lung Cell Mol Physiol 2013;305(1):L82-91.

67. Yang W, Hu P. Skeletal muscle regeneration is modulated by inflammation. Journal of Orthopaedic Translation 2018;13:25-32.

68. Puig-Vilanova E, Rodriguez DA, Lloreta J, et al. Oxidative stress, redox signaling pathways, and autophagy in cachectic muscles of male patients with advanced COPD and lung cancer. Free Radic Biol Med 2015;79:91-108.

69. Barreiro E, Peinado VI, Galdiz JB, et al. Cigarette smoke-induced oxidative stress: A role in chronic obstructive pulmonary disease skeletal muscle dysfunction. Am J Respir Crit Care Med 2010;182(4):477-88.

70. Puente-Maestu L, Lazaro A, Tejedor A, et al. Effects of exercise on mitochondrial DNA content in skeletal muscle of patients with COPD. Thorax 2011;66(2):121-7.

71. Schakman O, Kalista S, Barbe C, et al. Glucocorticoid-induced skeletal muscle atrophy. Int J Biochem Cell Biol 2013;45(10):2163-72.

72. Wang Y, Pessin JE. Mechanisms for fiber-type specificity of skeletal muscle atrophy. Current opinion in clinical nutrition and metabolic care 2013;16(3):243-50.

73. de Theije CC, Langen RC, Lamers WH, et al. Differential sensitivity of oxidative and glycolytic muscles to hypoxia-induced muscle atrophy. Journal of applied physiology 2015;118(2):20011.

74. Fermoselle $C$, Rabinovich $R$, Ausin $P$, et al. Does oxidative stress modulate limb muscle atrophy in severe COPD patients? Eur Respir J 2012;40(4):851-62.

75. Verdijk LB, Snijders T, Drost M, et al. Satellite cells in human skeletal muscle; from birth to old age. Age (Dordr) 2014;36(2):545-7.

76. Menon MK, Houchen L, Singh SJ, et al. Inflammatory and satellite cells in the quadriceps of patients with COPD and response to resistance training. Chest 2012;142(5):1134-42.

77. Theriault ME, Pare ME, Lemire BB, et al. Regenerative defect in vastus lateralis muscle of 
patients with chronic obstructive pulmonary disease. Respir Res 2014;15:35.

78. Theriault ME, Pare ME, Maltais F, et al. Satellite cells senescence in limb muscle of severe patients with COPD. PLoS One 2012;7(6):e39124.

79. Lakhdar R, Drost EM, MacNee W, et al. 2D-DIGE proteomic analysis of vastus lateralis from COPD patients with low and normal fat free mass index and healthy controls. Respir Res 2017;18(1):81.

80. Relaix F, Zammit PS. Satellite cells are essential for skeletal muscle regeneration: the cell on the edge returns centre stage. Development 2012;139(16):2845-56.

81. Lepper C, Partridge TA, Fan CM. An absolute requirement for Pax7-positive satellite cells in acute injury-induced skeletal muscle regeneration. Development 2011;138(17):3639-46.

82. Sambasivan R, Yao R, Kissenpfennig A, et al. Pax7-expressing satellite cells are indispensable for adult skeletal muscle regeneration. Development 2011;138(17):3647-56.

83. Murphy MM, Lawson JA, Mathew SJ, et al. Satellite cells, connective tissue fibroblasts and their interactions are crucial for muscle regeneration. Development 2011;138(17):3625-37.

84. Rosenblatt JD, Parry DJ. Gamma irradiation prevents compensatory hypertrophy of overloaded mouse extensor digitorum longus muscle. Journal of applied physiology 1992;73(6):2538-43.

85. Rosenblatt JD, Parry DJ. Adaptation of rat extensor digitorum longus muscle to gamma irradiation and overload. Pflugers Archiv : European journal of physiology 1993;423(34):255-64.

86. Fry CS, Lee JD, Jackson JR, et al. Regulation of the muscle fiber microenvironment by activated satellite cells during hypertrophy. FASEB journal : official publication of the Federation of American Societies for Experimental Biology 2014;28(4):1654-65.

87. Vogiatzis I, Simoes DC, Stratakos G, et al. Effect of pulmonary rehabilitation on muscle remodelling in cachectic patients with COPD. Eur Respir J 2010;36(2):301-10.

88. Vogiatzis I,Terzis G, Stratakos G, et al. Effect of pulmonary rehabilitation on peripheral muscle fiber remodeling in patients with COPD in GOLD stages II to IV. Chest 2011;140(3):744-52.

89. Constantin D, Menon MK, Houchen-Wolloff $L$, et al. Skeletal muscle molecular responses to resistance training and dietary supplementation in COPD. Thorax 2013;68(7):625-33.

90. Costes F, Gosker H, Feasson L, et al. Impaired exercise training-induced muscle fiber hypertrophy and Akt/mTOR pathway activation in hypoxemic patients with COPD. Journal of applied physiology 2015;118(8):1040-9.

91. Jones SE, Maddocks M, Kon SS, et al. Sarcopenia in COPD: prevalence, clinical correlates and response to pulmonary rehabilitation. Thorax 2015;70(3):213-8.

92. Barbalho MSM, Gentil P, Izquierdo M, et al. There are no no-responders to low or high resistance training volumes among older women. Exp Gerontol 2017;99:18-26.

93. Churchward-VenneTA, Tieland M,VerdijkLB, et al. There Are No Nonresponders to ResistanceType Exercise Training in Older Men and Women. J Am Med Dir Assoc 2015;16(5):400-11.

94. Pomies $\mathrm{P}$, Rodriguez J, Blaquiere $\mathrm{M}$, et al. Reduced myotube diameter, atrophic signalling and elevated oxidative stress in cultured satellite cells from COPD patients. J Cell Mol Med 2015;19(1):175-86.

95. Troosters T, Gosselink R, Decramer M. Short- and long-term effects of outpatient rehabilitation in patients with chronic obstructive pulmonary disease: a randomized trial. Am J Med 2000;109(3):207-12.

96. Guell R, Casan P, Belda J, et al. Long-term effects of outpatient rehabilitation of COPD: A randomized trial. Chest 2000;117(4):976-83.

97. Ries AL, Kaplan RM, Myers R, et al. Maintenance after pulmonary rehabilitation in chronic lung disease: a randomized trial. Am J Respir Crit Care Med 2003;167(6):880-8. 


\section{CHAPTER 8}

98. Swerts PM, Kretzers LM, Terpstra-Lindeman E, et al. Exercise reconditioning in the rehabilitation of patients with chronic obstructive pulmonary disease: a short- and longterm analysis. Arch Phys Med Rehabil 1990;71(8):570-3.

99. Strijbos JH, Postma DS, van Altena R, et al. A comparison between an outpatient hospitalbased pulmonary rehabilitation program and a home-care pulmonary rehabilitation program in patients with COPD. A follow-up of 18 months. Chest 1996;109(2):366-72.

100. Vale F, Reardon JZ, ZuWallack RL. The long-term benefits of outpatient pulmonary rehabilitation on exercise endurance and quality of life. Chest 1993;103(1):42-5.

101. MacMillan NJ, Kapchinsky S, Konokhova Y, et al. Eccentric Ergometer Training Promotes Locomotor Muscle Strength but Not Mitochondrial Adaptation in Patients with Severe Chronic Obstructive Pulmonary Disease. Frontiers in physiology 2017;8:114.

102. De Brandt J, Spruit MA, Derave W, et al. Changes in structural and metabolic muscle characteristics following exercise-based interventions in patients with COPD: a systematic review. Expert Rev Respir Med 2016;10(5):521-45.

103. Nederveen JP, Joanisse S, Snijders T, et al. The influence of capillarization on satellite cell pool expansion and activation following exercise-induced muscle damage in healthy young men. J Physiol 2018;596(6):1063-78.

104. Baehr LM, West DW, Marcotte G, et al. Age-related deficits in skeletal muscle recovery following disuse are associated with neuromuscular junction instability and ER stress, not impaired protein synthesis. Aging (Albany NY) 2016;8(1):127-46.

105. Jobin J, Maltais F, Doyon JF, et al. Chronic obstructive pulmonary disease: capillarity and fiber-type characteristics of skeletal muscle. J Cardiopulm Rehabil 1998;18(6):432-7.

106. Whittom F, Jobin J, Simard PM, et al. Histochemical and morphological characteristics of the vastus lateralis muscle in patients with chronic obstructive pulmonary disease. Medicine and science in sports and exercise 1998;30(10):1467-74.

107. Jatta K, Eliason G, Portela-Gomes GM, et al. Overexpression of von Hippel-Lindau protein in skeletal muscles of patients with chronic obstructive pulmonary disease. J Clin Pathol 2009;62(1):70-6.

108. Eliason G, Abdel-Halim SM, Piehl-Aulin K, et al. Alterations in the muscle-to-capillary interface in patients with different degrees of chronic obstructive pulmonary disease. Respir Res 2010;11:97.

109. Kelly NA, Hammond KG, Bickel CS, et al. Effects of aging and Parkinson's disease on motor unit remodeling: influence of resistance exercise training. Journal of applied physiology 2018;124(4):888-98.

110. Hiemstra PS. Altered macrophage function in chronic obstructive pulmonary disease. Ann Am Thorac Soc 2013;10 Suppl:S180-5.

111. Barnes PJ. Inflammatory mechanisms in patients with chronic obstructive pulmonary disease. J Allergy Clin Immunol 2016;138(1):16-27.

112. Yang W, Hu P. Skeletal muscle regeneration is modulated by inflammation. J Orthop Translat 2018;13:25-32.

113. Guttridge DC, Mayo MW, Madrid LV, et al. NF-kappaB-induced loss of MyoD messenger RNA: possible role in muscle decay and cachexia. Science 2000;289(5488):2363-6.

114. Langen RC, Van DerVelden JL, Schols AM, et al.Tumor necrosis factor-alpha inhibits myogenic differentiation through MyoD protein destabilization. FASEB journal : official publication of the Federation of American Societies for Experimental Biology 2004;18(2):227-37.

115. Cheek DB. The control of cell mass and replication. The DNA unit--a personal 20-year study. Early Hum Dev 1985;12(3):211-39.

116. Hall ZW, Ralston E. Nuclear domains in muscle cells. Cell 1989;59(5):771-2.

117. Kolesar JE, Safdar A, Abadi A, et al. Defects in mitochondrial DNA replication and oxidative 
damage in muscle of mtDNA mutator mice. Free Radic Biol Med 2014;75:241-51.

118. Yakes FM, Van Houten B. Mitochondrial DNA damage is more extensive and persists longer than nuclear DNA damage in human cells following oxidative stress. Proc Natl Acad Sci U S A 1997;94(2):514-9.

119. Herbst A, Widjaja K, Nguy B, et al. Digital PCR Quantitation of Muscle Mitochondrial DNA: Age, Fiber Type, and Mutation-Induced Changes. J Gerontol A Biol Sci Med Sci 2017;72(10):132733.

120. Wanagat J, Cao Z, Pathare $\mathrm{P}$, et al. Mitochondrial DNA deletion mutations colocalize with segmental electron transport system abnormalities, muscle fiber atrophy, fiber splitting, and oxidative damage in sarcopenia. FASEB journal : official publication of the Federation of American Societies for Experimental Biology 2001;15(2):322-32.

121. Wang X, Pickrell AM, Rossi SG, et al. Transient systemic mtDNA damage leads to muscle wasting by reducing the satellite cell pool. Hum Mol Genet 2013;22(19):3976-86.

122. Kyriakouli DS, Boesch P, Taylor RW, et al. Progress and prospects: gene therapy for mitochondrial DNA disease. Gene Ther 2008;15(14):1017-23.

123. Adhihetty PJ, Irrcher I, Joseph AM, et al. Plasticity of skeletal muscle mitochondria in response to contractile activity. Exp Physiol 2003;88(1):99-107.

124. Mishra P, Varuzhanyan G, Pham AH, et al. Mitochondrial Dynamics is a Distinguishing Feature of Skeletal Muscle Fiber Types and Regulates Organellar Compartmentalization. Cell Metab 2015;22(6):1033-44.

125. Shoubridge EA. Mitochondrial DNA diseases: histological and cellular studies. J Bioenerg Biomembr 1994;26(3):301-10.

126. Dumont NA, Wang YX, Rudnicki MA. Intrinsic and extrinsic mechanisms regulating satellite cell function. Development 2015;142(9):1572-81.

127. Chakkalakal J, Brack A. Extrinsic Regulation of Satellite Cell Function and Muscle Regeneration Capacity during Aging. J Stem Cell Res Ther 2012;Suppl 11:001.

128. Son JS, Chae SA, Testroet ED, et al. Exercise-induced myokines: a brief review of controversial issues of this decade. Expert Review of Endocrinology \& Metabolism 2018;13(1):51-58.

129. Forterre A, Jalabert A, Berger $E$, et al. Proteomic analysis of $\mathrm{C} 2 \mathrm{C} 12$ myoblast and myotube exosome-like vesicles: a new paradigm for myoblast-myotube cross talk? PLoS One 2014;9(1):e84153.

130. Forterre $A$, Jalabert $A$, Chikh $K$, et al. Myotube-derived exosomal miRNAs downregulate Sirtuin1 in myoblasts during muscle cell differentiation. Cell Cycle 2014;13(1):78-89.

131. Charge SB, Rudnicki MA. Cellular and molecular regulation of muscle regeneration. Physiol Rev 2004;84(1):209-38.

132. Huh JY. The role of exercise-induced myokines in regulating metabolism. Arch Pharm Res 2018;41(1):14-29.

133. Keipert S, Ost M, Johann K, et al. Skeletal muscle mitochondrial uncoupling drives endocrine cross-talk through the induction of FGF21 as a myokine. Am J Physiol Endocrinol Metab 2014;306(5):E469-82.

134. Villarroya J, Campderros L, Ribas-Aulinas F, et al. Lactate induces expression and secretion of fibroblast growth factor-21 by muscle cells. Endocrine 2018.

135. Ribas F, Villarroya J, Hondares E, et al. FGF21 expression and release in muscle cells: involvement of MyoD and regulation by mitochondria-driven signalling. Biochem $J$ 2014;463(2):191-9.

136. Liu X, Wang Y, Hou L, et al. Fibroblast Growth Factor 21 (FGF21) Promotes Formation of Aerobic Myofibers via the FGF21-SIRT1-AMPK-PGC1alpha Pathway. J Cell Physiol 2017;232(7):1893-906.

137. Liu X, Wang Y, Zhao S, et al. Fibroblast Growth Factor 21 Promotes C2C12 Cells Myogenic 


\section{CHAPTER 8}

Differentiation by Enhancing Cell Cycle Exit. Biomed Res Int 2017;2017:1648715.

138. Pawlikowski B, Vogler TO, Gadek K, et al. Regulation of skeletal muscle stem cells by fibroblast growth factors. Dev Dyn 2017;246(5):359-67.

139. Cornelison DD, Filla MS, Stanley HM, et al. Syndecan-3 and syndecan-4 specifically mark skeletal muscle satellite cells and are implicated in satellite cell maintenance and muscle regeneration. Dev Biol 2001;239(1):79-94. [published Online First: 2002/01/11]

140. Deng S, Azevedo M, Baylies M. Acting on identity: Myoblast fusion and the formation of the syncytial muscle fiber. Semin Cell Dev Biol 2017;72:45-55.

141. Sampath SC, Sampath SC, Millay DP. Myoblast fusion confusion: the resolution begins. Skelet Muscle 2018;8(1):3.

142. Giudice J, Taylor JM. Muscle as a paracrine and endocrine organ. Curr Opin Pharmacol 2017;34:49-55.

143. Gilbert PM, Havenstrite KL, Magnusson KE, et al. Substrate elasticity regulates skeletal muscle stem cell self-renewal in culture. Science 2010;329(5995):1078-81.

144. Gattazzo F, Urciuolo A, Bonaldo P. Extracellular matrix: a dynamic microenvironment for stem cell niche. Biochim Biophys Acta 2014;1840(8):2506-19.

145. Spinazzola JM, Gussoni E. Exosomal Small Talk Carries Strong Messages from Muscle Stem Cells. Cell Stem Cell 2017;20(1):1-3.

146. Fry CS, Kirby TJ, Kosmac K, et al. Myogenic Progenitor Cells Control Extracellular Matrix Production by Fibroblasts during Skeletal Muscle Hypertrophy. Cell Stem Cell 2017;20(1):5669.

147. Mackey AL, Magnan M, Chazaud B, et al. Human skeletal muscle fibroblasts stimulate in vitro myogenesis and in vivo muscle regeneration. J Physiol 2017;595(15):5115-27.

148. Segawa M, Fukada S, Yamamoto $Y$, et al. Suppression of macrophage functions impairs skeletal muscle regeneration with severe fibrosis. Exp Cell Res 2008;314(17):3232-44.

149. Furrer R, Eisele PS, Schmidt A, et al. Paracrine cross-talk between skeletal muscle and macrophages in exercise by PGC-1alpha-controlled BNP. Sci Rep 2017;7:40789.

150. Latroche C, Weiss-Gayet M, Muller L, et al. Coupling between Myogenesis and Angiogenesis during Skeletal Muscle Regeneration Is Stimulated by Restorative Macrophages. Stem Cell Reports 2017;9(6):2018-33.

151.Zordan P, Rigamonti E, Freudenberg K, et al. Macrophages commit postnatal endotheliumderived progenitors to angiogenesis and restrict endothelial to mesenchymal transition during muscle regeneration. Cell Death Dis 2014;5:e1031.

152. Chazaud B, Sonnet $C$, Lafuste $P$, et al. Satellite cells attract monocytes and use macrophages as a support to escape apoptosis and enhance muscle growth. J Cell Biol 2003;163(5):113343.

153. Ochoa O, Sun D, Reyes-Reyna SM, et al. Delayed angiogenesis and VEGF production in CCR2-/- mice during impaired skeletal muscle regeneration. Am J Physiol Regul Integr Comp Physiol 2007;293(2):R651-61.

154. Liu W, Klose A, Forman S, et al. Loss of adult skeletal muscle stem cells drives age-related neuromuscular junction degeneration. Elife 2017;6

155. Liu W, Wei-LaPierre L, Klose A, et al. Inducible depletion of adult skeletal muscle stem cells impairs the regeneration of neuromuscular junctions. Elife 2015;4

156. Dedkov El, Kostrominova TY, Borisov $A B$, et al. Reparative myogenesis in long-term denervated skeletal muscles of adult rats results in a reduction of the satellite cell population. Anat Rec 2001;263(2):139-54.

157. Jejurikar SS, Marcelo CL, Kuzon WM, Jr. Skeletal muscle denervation increases satellite cell susceptibility to apoptosis. Plast Reconstr Surg 2002;110(1):160-8. 
158. Ceelen JJM, Schols A, Thielen NGM, et al. Pulmonary inflammation-induced loss and subsequent recovery of skeletal muscle mass require functional poly-ubiquitin conjugation. Respir Res 2018;19(1):80.

159. Lopez-Otin C, Blasco MA, Partridge L, et al. The hallmarks of aging. Cell 2013;153(6):1194217.

160. Romanello V, Sandri M. Mitochondrial Quality Control and Muscle Mass Maintenance. Frontiers in physiology 2015;6:422.

161. Konokhova $Y$, Spendiff $S$, Jagoe RT, et al. Failed upregulation of TFAM protein and mitochondrial DNA in oxidatively deficient fibers of chronic obstructive pulmonary disease locomotor muscle. Skelet Muscle 2016;6:10.

162. Yue J, Guan J, Wang X, et al. MicroRNA-206 is involved in hypoxia-induced pulmonary hypertension through targeting of the HIF-1alpha/Fhl-1 pathway. Lab Invest 2013;93(7):74859.

163. Winbanks CE, Wang B, Beyer C, et al. TGF-beta regulates miR-206 and miR-29 to control myogenic differentiation through regulation of HDAC4. J Biol Chem 2011;286(16):13805-14.

164. Donaldson A, Natanek SA, Lewis A, et al. Increased skeletal muscle-specific microRNA in the blood of patients with COPD. Thorax 2013;68(12):1140-9.

165. Huang QK, Qiao HY, Fu MH, et al. MiR-206 Attenuates Denervation-Induced Skeletal Muscle Atrophy in Rats Through Regulation of Satellite Cell Differentiation via TGF-beta1, Smad3, and HDAC4 Signaling. Med Sci Monit 2016;22:1161-70.

166. Garg K, Boppart MD. Influence of exercise and aging on extracellular matrix composition in the skeletal muscle stem cell niche. Journal of applied physiology 2016;121(5):1053-58.

167. Cosgrove BD, Gilbert PM, Porpiglia E, et al. Rejuvenation of the muscle stem cell population restores strength to injured aged muscles. Nat Med 2014;20(3):255-64.

168. Loebel C, Burdick JA. Engineering Stem and Stromal Cell Therapies for Musculoskeletal Tissue Repair. Cell Stem Cell 2018;22(3):325-39.

169. Klimczak A, Kozlowska U, Kurpisz M. Muscle Stem/Progenitor Cells and Mesenchymal Stem Cells of Bone Marrow Origin for Skeletal Muscle Regeneration in Muscular Dystrophies. Arch Immunol Ther Exp (Warsz) 2018 

SUMMARY

SAMENVATTING 


\section{SUMMARY}

The progressive loss of skeletal muscle mass and function is frequently observed in elderly and patients with chronic diseases such as Chronic Obstructive Pulmonary Disease (COPD). This may lead to sarcopenia, which affects physical functioning and metabolic health. The prevention and treatment of sarcopenia requires a better understanding of the underlying cellular and molecular pathobiology. Although skeletal muscle catabolic processes have been broadly studied, a potential role for impaired skeletal muscle recovery in the development of sarcopenia is still underexplored. In this thesis, we therefore set out the development and use of novel strategies for the assessment of molecular disturbances in the regulation of skeletal muscle mass and quality in COPD patients, and discuss the implications for the development of COPDrelated muscle pathology.

To establish the current knowledge on the molecular alterations underlying muscle atrophy in COPD, we reviewed recent insights in the diagnosis and pathophysiology of muscle wasting in COPD (Chapter 2). Based on the available literature, we found indications for skeletal muscle molecular signaling towards increased protein degradation and protein synthesis, as well as increased myogenesis signaling, in muscle wasted COPD patients.

However, the available studies had either a limited sample size, or focused solely on either protein degradation or protein synthesis signaling. Furthermore, the role of myogenesis in muscle mass maintenance was incompletely assessed. To further establish the coordination and differential regulation of protein degradation and synthesis, as well as myogenesis, we analyzed an extensive panel of molecular regulators and mediators of these processes in skeletal muscle biopsies of a large, wellphenotyped cohort, and compared expression patterns in COPD patients with and without muscle wasting, and healthy controls (Chapter $\mathbf{3}$ ). While ubiquitin proteasome system (UPS) signaling was unaltered, indices of UPS regulation, autophagy signaling, and protein synthesis signaling were increased in COPD. These alterations were even more pronounced in muscle wasted COPD patients. Furthermore, myogenic signaling was increased in COPD, with no further alterations in muscle wasted COPD patients. We propose that this pattern of molecular alterations reflects ongoing muscle repair and remodeling. However, it remains unknown to what extent these alterations in basal skeletal muscle molecular signaling affect the coordination between anabolic signaling and the anabolic response required for skeletal muscle recovery after disuse. 
To identify and target factors that may hamper skeletal muscle recovery during resumed physical activity following disuse, a better understanding of the interrelationship between the regulation of muscle mass and metabolic plasticity during reloadinginduced recovery is imperative. We explored these relations by measuring a selected set of molecular regulators and mediators of muscle mass- and oxidative metabolismrelated processes, and studied their interrelationship during reloading-induced recovery following disuse in both humans and rodents (Chapter 4). We showed through the combination of a cluster analysis and high-resolution time-course study, that the molecular regulation of protein turnover, mitochondrial turnover, and myonuclear turnover are correlated and temporally associated during recovery from atrophy.

As these findings suggest an interdependency or joint regulation of these muscle mass- and oxidative metabolism-related processes during recovery from atrophy, we next used an unbiased integrative approach to identify groups of COPD patients with a differential skeletal muscle molecular response to pulmonary rehabilitation (Chapter 5). We identified two major patient clusters that differed in pulmonary rehabilitationinduced Autophagy, Myogenesis, Glucocorticoid signaling, and Oxidative metabolism regulation. Cluster 1 displayed a more pronounced change in marker expression than Cluster 2, which was accompanied by a more pronounced functional improvement upon pulmonary rehabilitation.

The in vivo studies in this thesis clearly indicated a coordinated regulation of myogenesis during muscle mass and metabolic plasticity, and provided indications for altered myogenesis signaling in COPD patients. To further elucidate the role and regulation of postnatal myogenesis in skeletal muscle mass and metabolic plasticity, we developed an in vitro model that better reflects in vivo postnatal myonuclear accretion (Chapter 6). This yielded a validated, physiologically relevant, sensitive, high-throughput, Cre/ LoxP-based cell system to semi-quantitatively assess in vitro postnatal myonuclear accretion by the conditional expression of luciferase upon myoblast-myotube fusion. Next, we used this in vitro model to study the role and regulation of postnatal myogenesis in non-hypertrophic skeletal muscle adaptation and recovery (Chapter 7). We showed that recovery from myotube metabolic stress or atrophy increased in vitro postnatal myonuclear accretion, and found indications that, specifically during recovery from metabolic stress, this is mediated by AMPK/FGF21 signaling.

In the final chapter, we address the implications of the findings presented in this thesis, and define important goals for research in this area (Chapter $\mathbf{8}$ ). The studies described 
in this thesis collectively indicate that postnatal myogenesis plays an important role in the regulation of muscle mass and metabolic plasticity, and that dysregulation of this process may be central in the development of COPD-related muscle pathology. To date, exercise training remains the most comprehensive and effective intervention to improve skeletal muscle mass and function in COPD patients. However, to better understand the variability in the exercise/rehabilitation response and the association with the progressive loss of muscle mass and function, future studies should include the characterization of baseline differences, and the sequential rehabilitation-induced modification of satellite cell niche components in patients with COPD. Thereby, we may be able to select training modalities, potentially with other co-interventions, that most potently improve the rehabilitation response in specific patient groups. 


\section{SAMENVATTING}

Sarcopenie is een syndroom dat gekenmerkt wordt door verlies van massa en functie van de skeletspieren en dat veel voorkomt bij ouderen en bij patiënten met de chronische longziekte COPD. Om sarcopenie te kunnen voorkomen en behandelen is een beter begrip van de onderliggende cellulaire en moleculaire mechanismen vereist. Eerder spierbiologisch onderzoek richtte zich vooral op afbraakprocessen. Een mogelijke rol voor een verstoord skeletspierherstel na spiermassa verlies in de ontwikkeling van sarcopenie is tot op heden echter onderbelicht. In dit proefschrift passen we nieuwe strategieën toe voor de bestudering van moleculaire stoornissen in de regulatie van skeletspiermassa en -kwaliteit bij COPD-patiënten.

Allereerst hebben we een literatuuronderzoek gedaan naar de meest recente inzichten in de diagnose en pathofysiologie van COPD-gerelateerd spiermassaverlies (Hoofdstuk 2). Op basis van de beschikbare literatuur vonden we aanwijzingen voor een moleculaire signalering duidend op een verhoogde spiereiwitafbraak en spiereiwitaanmaak, alsook een verhoogde signalering van de ontwikkeling van spierweefsel, de zogenaamde myogenese, bij COPD-patiënten met een lage spiermassa. De beschikbare studies hadden echter een beperkte steekproefomvang, of bestudeerden enkel eiwitaanmaak dan wel eiwitafbraak signalering. Daarnaast werd de rol van myogenese in het behoud van spiermassa in deze studies onderzocht met behulp van een zeer beperkt aantal moleculaire markers.

Om de afstemming en veranderde regulatie van eiwitafbraak en -aanmaak alsook myogenese in COPD patiënten verder te onderzoeken, hebben we een uitgebreid panel van moleculaire regulatoren en mediatoren van deze processen bestudeerd in stukjes spierweefsel van een grote groep COPD patiënten. Hierin vergeleken we de expressiepatronen van deze markers tussen COPD-patiënten met een normale en een lage spiermassa, alsook met die van gezonde proefpersonen (Hoofdstuk 3). Hoewel er geen grote verschillen vastgesteld werden in expressie van componenten van de afbraakmachinerie, waaronder het ubiquitine proteasoom systeem (UPS) en autofagie, duidden de markers van regulatie op een verhoogde capaciteit om deze processen te kunnen activeren in de spier van COPD patiënten. Daarnaast was ook de eiwitaanmaak signalering verhoogd in COPD patiënten. De veranderingen in eiwitafbraak en eiwitaanmaak signalering waren zelfs nog meer uitgesproken in COPD-patiënten met een lage spiermassa. Bovendien was de myogenese signalering verhoogd in zowel COPD-patiënten met normale spiermassa als die met een lage spiermassa. We suggereren dat dit patroon van moleculaire veranderingen de 
aanwezigheid van voortdurende spierreparatie en -remodelering reflecteert. Het blijft echter onbekend in hoeverre deze basale veranderingen in de moleculaire signalering van invloed zijn op de afstemming tussen de anabole signalering en de daadwerkelijke anabole respons die nodig is voor skeletspierherstel na inactiviteit.

Veranderingen in fysieke activiteit gaan, naast veranderingen in spiermassa, gepaard met aanpassingen in het energiemetabolisme. Om belemmerende factoren voor het herstel van skeletspieren na fysieke inactiviteit te kunnen identificeren en gericht te kunnen beïnvloeden, dienen we de onderlinge relatie tussen de regulatie van spiermassa en metabole plasticiteit tijdens spierherstel beter te begrijpen. We hebben dit onderzocht door, in zowel mensen als muizen, geselecteerde moleculaire regulatoren en mediatoren te meten die een rol spelen in de regulatie van spiermassa en oxidatief metabolisme, en vervolgens hun onderliggende samenhang te bestuderen tijdens spierherstel na inactiviteit (Hoofdstuk 4). Door middel van een clusteranalyse, gecombineerd met een gedetailleerde analyse van het verloop in de tijd, toonden we aan dat de moleculaire regulatie van de vervanging van eiwitten, mitochondriën en spiervezelkernen, samenhang vertoont tijdens het herstel van atrofie.

Deze bevindingen duiden op een onderliggende afhankelijkheid of gedeelde regulatie van spiermassa- en oxidatief metabolisme gerelateerde processen tijdens het herstel van atrofie. Daarom hebben we vervolgens een objectieve, integrerende statistische benadering gebruikt om clusters van COPD-patiënten te onderscheiden die van elkaar verschilden in de moleculaire skeletspierrespons op longrevalidatie (Hoofdstuk 5). Hiermee werden twee belangrijke patiënt clusters onderscheiden, welke verschilden in de revalidatie-geïnduceerde regulatie van Autofagie, Myogenese, Glucocorticoïd signalering en Oxidatief metabolisme. Cluster 1 vertoonde een meer uitgesproken verandering in expressiepatronen dan Cluster 2, wat tevens gepaard ging met een meer uitgesproken functionele verbetering na longrevalidatie.

De studies beschreven in dit proefschrift wijzen duidelijk op een gecoördineerde regulatie van myogenese tijdens actieve veranderingen in spiermassa en metabolisme, en wijzen op een veranderde myogenese signalering in COPDpatiënten. Om de rol en regulatie van postnatale myogenese in de plasticiteit van skeletspiermassa en -metabolisme verder te doorgronden, hebben we een celkweek model ontwikkeld dat de postnatale aanwas van spiervezelkernen die in de spier plaatsvindt beter weerspiegelt (Hoofdstuk 6). We ontwikkelden en valideerden een genetisch gemodificeerd celkweeksysteem waarmee we postnatale celkernaanwas semi-kwantitatief kunnen bepalen door het meten van de conditionele expressie 


\section{Samenvatting}

van een marker-enzym na de fusie van spierstamcellen met gekweekte spiervezels. Deze efficiënte, fysiologisch relevante, en gevoelige methode hebben we vervolgens gebruikt om de rol en regulatie van postnatale myogenese in skeletspieradaptatie en -herstel te bestuderen (Hoofdstuk 7). In dit hoofdstuk hebben we aangetoond dat het herstel van spiervezels niet enkel na atrofie, maar ook na metabole stress, gepaard gaat met een verhoogde postnatale aanwas van spiervezelkernen. Bovendien vonden we aanwijzingen dat, specifiek tijdens het herstel van metabole stress, dit gemedieerd wordt door AMPK/FGF21-signalering.

In het laatste hoofdstuk bespreken we de implicaties van de bevindingen gepresenteerd in dit proefschrift, en definiëren we aandachtspunten voor vervolgonderzoek (Hoofdstuk 8). Samen tonen de studies beschreven in dit proefschrift aan dat postnatale myogenese een belangrijke rol speelt in de regulatie van spiermassa en metabole plasticiteit, en dat ontregelingen van deze processen mogelijk bijdragen aan de ontwikkeling van COPD-gerelateerde spierpathologie. Tot op heden blijft fysieke training de meest omvattende en effectieve interventie om de skeletspiermassa en -functie bij COPD-patiënten te verbeteren. Echter, voor een beter begrip van de variabiliteit van de respons op inspanning en longrevalidatie, en hoe deze bijdraagt aan het progressieve verlies van spiermassa en -functie in patiënten met COPD, zou toekomstig onderzoek zich moeten richten op het karakteriseren van basale verschillen, en verschillen in de tijdsafhankelijke revalidatie-geïnduceerde aanpassingen van componenten van de spierstamcel omgeving. Met deze kennis kunnen we in de toekomst mogelijk de revalidatie respons verbeteren, door de trainingsmodaliteiten af te stemmen op specifieke patiëntgroepen of te combineren met aanvullende behandelingen. 
VALORIZATION 
The aging of the world's population is one of the main forces driving the development and the increasing burden of chronic diseases such as chronic obstructive pulmonary disease (COPD), of which the prevalence is rising and which is already the third leading cause of death worldwide [1]. Interestingly, loss of lung function is one of the characteristics of 'normal' aging, and was already reported by Falzone et al., in 1956 [2]. Overall, aging is characterized by changes in physiological structure and function, of which, Rosenberg noted, the loss of muscle mass is perhaps the most striking [3-5]. To describe this phenomenon, Rosenberg proposed the term 'sarcopenia' in 1989 (From the Greek words 'sarx' (flesh) and 'penia' (loss)) [6].

While, to date, there is still no international consensus on the diagnostic criteria for sarcopenia, several working groups have developed objective definitions of sarcopenia, all including both a low muscle mass together with low muscle function (strength or performance) [7-11]. Depending on the definition used, the prevalence of sarcopenia in $60-70$ year olds is $5-13 \%$, and rises to $11-50 \%$ in > 80 year olds [12]. However, sarcopenia is not only a disease of the elderly, but is also associated with chronic diseases such as COPD, and is an integral part of the disease-related wasting syndrome called 'cachexia' [11]. Interestingly, compared to the same age group, the prevalence of sarcopenia is significantly higher in COPD patients, reaching $14-40 \%$ in 60-70 year olds $[13,14]$. Importantly, besides that sarcopenia is an adverse health outcome and is associated with chronic diseases, it is also an independent risk factor for other adverse outcomes such as cognitive decline, injurious falls, and loss of independence, and is a powerful predictor of mortality [14-17]. Together, this has led to the recent recognition of sarcopenia as an independent disease entity with an ICD10-CM (M62.84) code [18].

Prominent researchers in the field of sarcopenia have predicted that the recognition of sarcopenia as an independent disease will lead to an accelerated development and availability of diagnostic tools, as well as a growing interest by physicians in integrating the diagnostic assessment of sarcopenia in routine clinical practice [18]. Furthermore, the recognition of sarcopenia as an independent disease is expected to increase the interest of pharmaceutical and nutritional companies in developing drugs and medical nutrition to treat sarcopenia [18]. This process is nicely illustrated in a 'from bench to bedside' translational research continuum (Figure 1), which also emphasizes the importance of adequate observation and recognition of the disease in the process. 


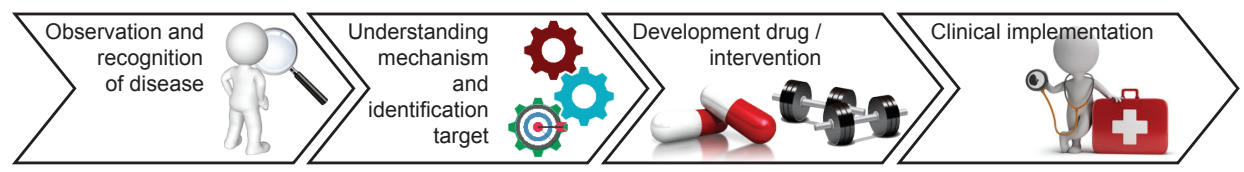

Figure 1. | Translational research continuum

Importantly, the etiology of sarcopenia is multi-factorial [7, 12]. Added to the wellestablished heterogeneity of the COPD population, it is evident that, next to generic interventions such as stimulating a physically active lifestyle, targeted primary and secondary prevention of sarcopenia in COPD patients is not easy. Adequate early intervention and the development of tailored treatment plans for sarcopenia in COPD patients requires the identification of more homogeneous risk populations, e.g. by sub-classification of endotypes, as a first step in the translational research continuum. Given the complexity of the disease, an integrated and unbiased approach is required to identify relevant endotypes. In chapter 5 of this thesis, we used an unbiased clustering-based approach to address the heterogeneity in the molecular rehabilitation responses that may be underlying to a progressive decline in muscle mass and function. Through this novel approach, we identified two major patient clusters which were characterized by an 'early' versus 'late' stage molecular response pattern to PR. This opens up avenues for personalization of the intensity and duration of PR, and for selective additional nutritional and pharmaceutical interventions based on the molecular response pattern. Another important observation was that no cluster of non-responders was identified, illustrating the importance of PR, when feasible, as primary approach to treat sarcopenia in advanced COPD before considering additional pharmaceutical or nutritional interventions. These findings are of relevance to medical and paramedical caregivers, medical decision and policy makers and health insurance companies.

The integrative analysis of molecular data, adopted in both chapters 4 and 5, can also be applied to different research fields and to answer various types of research questions. For example, such an approach can be used by fundamental researchers to gain more insight in potential molecular alterations in highly dynamic processes, such as cellular proliferation and differentiation. Furthermore, it can be used in a clinical (research) setting to identify distinct endotypes that respond differentially to treatment, to improve accurate diagnosis, develop specific drugs, nutraceuticals, and lifestyle interventions, and/or provide a targeted treatment to the appropriate patient group to improve cost-effectiveness. 
To date, the most effective treatment for sarcopenia is resistance-type exercise training, which induces protein turnover signaling to facilitate skeletal muscle remodeling. To promote a gain in muscle mass, it is frequently noted that additional pharmacological anabolic triggers, can be used to combat the blunted anabolic response to exercise training in elderly and COPD patients. However, this thesis shows in chapter 3 that anabolic signaling is already increased at baseline in muscle wasted COPD patients. Based on this, we could speculate that pharmacological anabolic stimuli may have a limited effect in this population, reducing cost-effectiveness of such treatments. Nevertheless, several studies showed that anabolic stimuli such as recombinant growth hormone [19], anabolic steroids [20-23], and an Activin type II receptor blocker [24], did induce or promote a gain in muscle mass in COPD patients. Importantly, these studies collectively showed no improvements in muscle function, indicating that increasing muscle mass alone is not sufficient to promote exercise performance. This dissociation between improvements in muscle mass and muscle function may be due to their respective determination by the balance versus the rate of muscle protein turnover, as discussed in chapter 8 . Indeed, these anabolic stimuli promote a positive protein turnover balance, but do not necessarily increase the rate of protein turnover. Importantly, these anabolic stimuli signal through the PI3K-AKT1 pathway, which may even result in a decrease in the protein turnover rate by suppression of protein degradation. As such, these anabolic stimuli may inhibit muscle repair and remodeling, which are required for the recovery and maintenance of muscle quality and function. In chapter 3 , we showed that in addition to the increased baseline protein synthesis signaling, protein degradation signaling was also increased in muscle wasted COPD patients, suggesting ongoing skeletal muscle repair and remodeling. This information is of value for rehabilitation physicians, as it demands caution with general implementation of additional anabolic stimuli in exercise-based pulmonary rehabilitation. Nevertheless, a supportive environment for anabolism is important for improvement of both muscle mass and muscle function, emphasizing the requirement for integrating nutritional support as part of PR.

Furthermore, in this thesis we report several indications for alterations in postnatal myogenesis in COPD patients. However, studying the individual processes involved in postnatal myogenesis is not yet feasible in vivo, and even difficult in vitro. In chapter 6, we describe the development of a novel in vitro model to efficiently study postnatal myogenesis. This model can be used by basic researchers to further unravel the molecular mechanisms involved in postnatal myogenesis, which may lead to the discovery of in vivo applicable biomarkers or drug targets. Moreover, the model can be used as a screening tool for potential nutritional or pharmaceutical compounds that 
promote muscle mass and quality, and thereby contribute to a more efficient drug or intervention development. In addition, in vitro (pre)screening of compounds will reduce the need for animal studies, reducing research costs, as well as contributing to the 3R's of animal research. Moreover, fundamental biological insights obtained using this in vitro system are likely relevant for the optimization of stem cell-based therapies. Optimization of such therapies will not only be important for the field of aging and chronic disease related muscle pathologies, but also for the treatment of muscle dystrophies.

In conclusion, this thesis provides both new scientific insights, as well as tools for further knowledge development. This will support crucial steps in the translational research continuum, hopefully contributing to a future with sarcopenia as a treatable, and maybe even preventable, disease. 


\section{REFERENCES}

1. World Health Organization. Fact sheets: The top 10 causes of death 2018 [15 August 2018]. Available from: http://www.who.int/news-room/fact-sheets/detail/the-top-10-causes-ofdeath.

2. Falzone JA, Jr., Landowne $M$, Norris $A H$, et al. Pulmonary function studies: age differences in lung volumes and bellows function. J Gerontol 1956;1 1(4):379-87.

3. Rowe JW, Andres R, Tobin JD, et al. The effect of age on creatinine clearance in men: a crosssectional and longitudinal study. J Gerontol 1976;31(2):155-63.

4. Forbes GB, Reina JC. Adult lean body mass declines with age: some longitudinal observations. Metabolism 1970;19(9):653-63.

5. Rosenberg IH. Sarcopenia: origins and clinical relevance. J Nutr 1997;127(5 Suppl):990S-91S.

6. Rosenberg $\mathrm{IH}$. Summary comments. The American Journal of Clinical Nutrition 1989;50(5):1231-33.

7. Cruz-Jentoft AJ, Baeyens JP, Bauer JM, et al. Sarcopenia: European consensus on definition and diagnosis: Report of the European Working Group on Sarcopenia in Older People. Age Ageing 2010;39(4):412-23.

8. Fielding RA, Vellas B, Evans WJ, et al. Sarcopenia: an undiagnosed condition in older adults. Current consensus definition: prevalence, etiology, and consequences. International working group on sarcopenia. J Am Med Dir Assoc 2011;12(4):249-56.

9. Morley JE, Abbatecola AM, Argiles JM, et al. Sarcopenia with limited mobility: an international consensus. J Am Med Dir Assoc 2011;12(6):403-9.

10. Dam TT, Peters KW, Fragala M, et al. An evidence-based comparison of operational criteria for the presence of sarcopenia. J Gerontol A Biol Sci Med Sci 2014;69(5):584-90.

11. Muscaritoli M, Anker SD, Argiles J, et al. Consensus definition of sarcopenia, cachexia and pre-cachexia: joint document elaborated by Special Interest Groups (SIG) "cachexiaanorexia in chronic wasting diseases" and "nutrition in geriatrics". Clin Nutr 2010;29(2):1549.

12. Santilli V, Bernetti A, Mangone $M$, et al. Clinical definition of sarcopenia. Clin Cases Miner Bone Metab 2014;11(3):177-80.

13. Schols AM, Soeters PB, Dingemans AM, et al. Prevalence and characteristics of nutritional depletion in patients with stable COPD eligible for pulmonary rehabilitation. Am Rev Respir Dis 1993;147(5):1151-6.

14. Vestbo J, Prescott E, Almdal T, et al. Body mass, fat-free body mass, and prognosis in patients with chronic obstructive pulmonary disease from a random population sample: findings from the Copenhagen City Heart Study. Am J Respir Crit Care Med 2006;173(1):79-83.

15. Mostert R, Goris A, Weling-Scheepers $C$, et al. Tissue depletion and health related quality of life in patients with chronic obstructive pulmonary disease. Respir Med 2000;94(9):859-67.

16. Engelen MP, Schols AM, Baken WC, et al. Nutritional depletion in relation to respiratory and peripheral skeletal muscle function in out-patients with COPD. Eur Respir J 1994;7(10):17937.

17. Schols AM, Broekhuizen R, Weling-Scheepers CA, et al. Body composition and mortality in chronic obstructive pulmonary disease. Am J Clin Nutr 2005;82(1):53-9.

18. Anker SD, Morley JE, von Haehling S. Welcome to the ICD-10 code for sarcopenia. J Cachexia Sarcopenia Muscle 2016;7(5):512-14.

19. Burdet $L$, de Muralt $B$, Schutz $Y$, et al. Administration of growth hormone to underweight patients with chronic obstructive pulmonary disease. A prospective, randomized, controlled study. Am J Respir Crit Care Med 1997;156(6):1800-6. 
20. Ferreira IM, Verreschi IT, Nery LE, et al. The influence of 6 months of oral anabolic steroids on body mass and respiratory muscles in undernourished COPD patients. Chest 1998;114(1):1928.

21. Yeh SS, DeGuzman B, Kramer T, et al. Reversal of COPD-associated weight loss using the anabolic agent oxandrolone. Chest 2002;122(2):421-8.

22. Casaburi R, Bhasin S, Cosentino $L$, et al. Effects of testosterone and resistance training in men with chronic obstructive pulmonary disease. Am J Respir Crit Care Med 2004;170(8):870-8.

23. Schols AM, Soeters PB, Mostert R, et al. Physiologic effects of nutritional support and anabolic steroids in patients with chronic obstructive pulmonary disease. A placebocontrolled randomized trial. Am J Respir Crit Care Med 1995;152(4 Pt 1):1268-74.

24. Polkey MI, Praestgaard J, Berwick A, et al. Activin Type II Receptor Blockade for Treatment of Muscle Depletion in COPD: A Randomized Trial. Am J Respir Crit Care Med 2018 

ACKNOWLEDGMENTS 


\section{Acknowledgments}

You are reading this chapter, which means that I have finished my thesis. While it is evident that a thesis is more than just a collection of research papers, I want to emphasize that a $\mathrm{PhD}$ studentship has more output than a thesis. I am indebted to many people who, in one way or another, have contributed to my personal and academic development leading to the successful completion of my PhD. In particular, I would like to thank a number of people, in my native language.

Als eerst wil ik mijn promotor Annemie bedanken voor het bieden van de mogelijkheid om binnen de vakgroep Longziekten te promoveren. Ik heb veel respect voor de manier waarop jij je verschillende taken binnen en buiten de universiteit weet te combineren, met behoud van een aanstekelijk enthousiasme voor onderzoek. Onder andere door mij te stimuleren om abstracte onderzoeks-ideeën of -concepten te concretiseren heb je mijn ambitie gevoed om mijn werk als een Postdoc te vervolgen.

Uiteraard wil ik ook mijn copromotoren bedanken.

Ramon, jij nam de dagelijkse begeleiding op je tijdens mijn PhD. Bedankt dat je deur altijd open stond om problemen of zorgen te bespreken, maar ook — niet het minst — om enthousiast binnen te huppelen met nieuwe onderzoeksresultaten. Jij weet als geen ander een veilige leeromgeving te creëren. Mede hierdoor kijk ik in het bijzonder met veel plezier terug op de meetings waarin we samen brainstormden over hypothesen en alternatieve hypothesen.

Lex, je bent nuchter en efficiënt, en was wat mij betreft daarmee de ideale persoon om mijn promotieteam te completeren. Zeker in de latere jaren van mijn PhD kon ik jouw 'niet lullen maar poetsen' mentaliteit erg waarderen. Jouw kritische blik op gerapporteerde fysiologische responsen ('hoe gevoelig kun je satelliet-cel aantallen nu eigenlijk meten?') heeft mij veel geleerd, wat ook waardevol bleek voor de ontwikkeling van het in vitro model. Bedankt voor al je uitstapjes naar de 5 e en voor het feit dat ook jouw deur altijd open stond, zelfs voor wanneer het over mijn (suffe) celkweekjes ging.

Luc, als officieus lid van mijn promotieteam wil ik ook jou graag bedanken. Je hebt meerdere pogingen gedaan om mij uit het celkweek-lab te lokken, maar ik voel mij daar voorlopig nog op mijn plek. Bedankt voor alle feedback en wijze raad. Met jouw stem in mijn achterhoofd verwacht ik ook in de toekomst nooit de fysiologische relevantie (of het gebrek daaraan) van in vitro bevindingen uit het oog te verliezen.

I would like to thank my committee for reading and approving my thesis, and for being present at my defence.

Uiteraard wil ik ook mijn paranimfen Rosanne en Pieter bedanken. Ik weet zeker dat het vertrouwd zal voelen om mijn thesis te verdedigen met jullie aan mijn zijden — een opstelling vergelijkbaar aan onze (vele) koffie-haal-wandelingen. 
Rosanne, je bent het beste kamergenootje dat ik ooit bij PUL heb gehad! We hebben op kamer 5.320 de afgelopen jaren veel zin en onzin gedeeld, wat er voor zorgde dat ik altijd zin had om naar het werk te gaan. Bedankt dat je mijn klankbord was voor werk-gerelateerde en persoonlijke kwesties, en voor alle gezelligheid en hilariteit tijdens het werk, het fitnessen, het shoppen, en het consumeren van biertjes en wijntjes. Er zijn een aantal woorden die ik niet meer zonder echo kan horen, en daarmee weet ik zeker dat ik je ook niet vergeet zodra ik niet meer werk in M-S-T-R-zachte G. Laten we proosten op de stapavonden die waar-dan-ook nog mogen volgen.

Pieter, kritisch denken — zoals het goede wetenschappers betaamt — zit jou in het bloed, en daarmee hield je mij scherp. Misschien vallen de vragen van de corona zelfs wel mee na alle terechte en onterechte kritische vragen die je mij in de loop van de jaren hebt voorgeschoteld. Deze scherpzinnigheid draagt bij aan jouw prettige relativeringsvermogen, en het feit dat wij om eigenlijk alles kunnen lachen. Ik weet zeker dat je een mooie carrière te wachten staat, en ik kijk er naar uit om in de toekomst weer samen te werken. Ik wil jou, maar natuurlijk ook je vrouw Judith, bedanken voor de gezelligheid tijdens het terrassen, borrelen, barbecueën en de vakantie in Frankrijk. Judith, je was al 'senior' PhD toen ik bij PUL kwam werken, en je assertieve en ietwat sceptische houding vond ik als 'junior' misschien wel wat intimiderend. Al snel kwam ik er echter achter dat je een lieverd bent, en onder het motto'alles is gemakkelijk als je weet hoe het moet' oneindig veel geduld hebt om dingen uit te leggen en voor te doen. Bijvoorbeeld hoe je een bommetje moet maken van een epje en droogijs ;). Het was leuk om jou en Pieter te zien ontwikkelen van pril verliefd stelletje naar man en vrouw. Uiteraard staat het bier altijd koud om de nieuwste ontwikkeligen in het leven, of ongerelateerde onzin, te bespreken.

Daarnaast zijn er een aantal collega's die bij hebben gedragen aan de totstandkoming van de wetenschappelijke artikelen beschreven in dit proefschrift, en de succesvolle afronding van mijn PhD. Chiel, zonder jouw hulp was ik waarschijnlijk nu nog steeds aan het cloneren. $\mathrm{Na}$ het horen van je naam zul je dat ongetwijfeld in je hoofd aanvullen met 'heb je tijd voor een vraagje?'. Je had nooit tijd, maar maakte het altijd. Met jou als achterwacht kreeg ik de kans om steeds meer ervaring en zelfvertrouwen in het lab op te doen. Bedankt voor alles wat je me hebt geleerd, voor de brainstormsessies, en bovenal voor de gezelligheid! Marco, ik denk dat als je al onze gekweekte spiervezeltjes optelt, we toch zeker een flink biefstuk hebben gemaakt. Bedankt voor alle experimenten en analyses die je voor me hebt gedaan, waardoor ik tijdens de afronding van mijn PhD mij met een gerust hart kon focussen op het schrijven van mijn thesis. Ik hoop dat we ook in de komende tijd samen nog mooie data kunnen verzamelen. Karin, wij hebben samen de eerste stapjes in het wetenschappelijk publiceren gezet. Bedankt voor je kritische vragen tijdens de labmeetings en voor je interesse in mijn werk. Veel succes met de laatste loodjes, en de nieuwe uitdagingen in de kliniek. Nicky en Evelien, bedankt dat ik jullie mooie studies mocht (her)gebruiken voor het beantwoorden van nieuwe vraagstellingen. Janneau, Rachel en Antoine, bedankt voor de technische ondersteuning vanuit BW, en voor 


\section{Acknowledgments}

het aanleren van de satellietcel staining en analyse. Tim, bedankt voor de fijne gesprekken over het onderzoek en voor de carrièretips. Ik hoop dat je een mooie onderzoeklijn kunt uitwerken in Maastricht, en dat we in de toekomst nog eens nauwer kunnen samenwerken. Mitja, thank you for the fruitful collaboration during my PhD. I would also like to thank all other co-authors for their time and critical remarks on the manuscripts.

During my PhD, I had the privilege to co-supervise several bright students. Mark, Ellis, Cristina, and Leonor, thank you for your critical questions, your hard work, and your enthusiasm for science. I hope I was able to teach you something, but you have definitely taught me a lot.

Daarnaast wil ik graag alle collega's en oud-collega's bedanken voor de gezellige jaren bij pulmonologie. Alex, door jou voelde ik mij onmiddelijk welkom bij PUL. Je stond met open armen klaar om mij de kneepjes van het vak (=bier drinken) te leren. Bedankt voor alles waarmee het bier drinken gepaard ging; barbequen, dansen, en de goede gesprekken. Wouter en Wessel, jullie hebben jezelf ongeveer een jaar geleden tot activiteitencommissie gebombardeerd. Teleurstellend genoeg is er nog niet veel van terecht gekomen. Gelukkig heb ik mijzelf af en toe uitgenodigd voor een diner of spelletjesavond, waardoor het toch nog goed kwam. Bedankt voor alle gezelligheid! Wouter, succes met je PhD. Met jouw ambitie komt dat vast helemaal goed. Wessel, jij succes gewenst met de afronding van je PhD. Tegen die tijd kom ik graag langs om er een alcoholvrije versnapering op te drinken. Harry, het is verfrissend om te zien hoe jij als doorgewinterde wetenschapper nooit blind meegaat in ongefundeerde tradities. Bedankt voor al je input en hulp, zowel tijdens de labmeetings als daarbuiten. Bedankt ook voor je gastvrijheid en de gezellige avonden rond de vuurkorf in je achtertuin. Sarah, thank you for introducing me in the Canadian culture. Unfortunately, I have not been able to use this knowledge yet, but I will certainly drop by for a refresher course when needed. Jules en Niki, bedankt voor jullie waardevolle input en feedback tijdens het schrijven van mijn eerste onderzoeks-aanvraag. Dames van de longlijn (Mieke, Niki, Juanita), en natuurlijk ook Caspar, Cheryl, Coby, Rianne, Martijn, Viviënne, Juliette, Lisanne, Bregtje en Vasili, bedankt voor jullie feedback tijdens de labmeetings, de gezelligheid tijdens de borrels en labuitjes, en jullie bijdrage aan de fijne sfeer bij PUL.

Vrienden, familie en schoonfamilie zijn absoluut onmisbaar tijdens een promotietraject. Zonder jullie was mijn periode als PhD student ongetwijfeld een stuk minder aangenaam en waarschijnlijk ook minder succesvol geweest.

Annemieke, je hebt mij zien ontwikkelen van stuiterbal naar een (semi)gefocuste student. Bedankt ook voor je moederlijke aanmoedigingen, die er voor zorgden dat ik zelfvertrouwen had voor de (toch wel spannende) toetsen, en om de overstap van HBO, naar Universiteit, naar een $\mathrm{PhD}$ te maken. Dominique, Manon, Charlotte, Sylvie, Bianca, zonder jullie zou mijn master tijd lang niet zo fijn zijn geweest. Het is leuk om te merken hoe de gesprekken tijdens onze dinertjes 
zijn veranderd. Bedankt voor alle gezelligheid en steun de afgelopen jaren, en ik kijk uit naar alle dinertjes, (promotie)feestjes, bruiloften en babyshowers die ongetwijfeld nog gaan komen. Een verhuizing naar een nieuwe stad is altijd spannend, maar Julie en Maarten, het scheelde een hoop dat jullie ook net in Maastricht waren neergestreken. Zo konden we samen een beetje de cultuurshock te boven komen. Bedankt voor de borrel-diners, de naailessen, het fitnessen, en de gezellige hardlooprondes met 'die gekke Maastrichtenaren'. Dennis, bedankt dat je (samen met Marcel) Julie en mij mee nam voor heel wat hardloop rondes door de pratsj. Ik kijk met veel plezier terug op de (ongewenste) modderbaden, onze sprongen in de Maas, en je veel te harde knuffels. Andrea, we go waaaay back. Onze vriendschap ontstond als een soort van liefde op het eerste gezicht, en is sindsdien altijd vanzelfsprekend geweest. Ik ben er dan ook zeker van dat wij elkaar als bejaarden nog steeds zullen opzoeken. Bedankt dat je mij altijd bewust houd van de dingen die écht belangrijk zijn in het leven, maar ook dat je mijn oneindige hoeveelheid bullshit altijd met geduld aanhoort. Ik wens je met Martijn een fantastische toekomst!

Lieve grote broer, Erik, ik ken niemand die zo leergierig is als jij. Bedankt voor je oprechte interesse in mijn werk, en dat je altijd het geduld hebt om mij opnieuw uit te leggen wat jij nu eigenlijk doet. Ik heb mij er inmiddels bij neergelegd dat ik nooit zo slim zal zijn als jij, maar voor jou ben ik vanaf nu wel doctor dom zusje. Ik wens je samen met Marije alle geluk toe daar in het hoge noorden. Linda, mijn kleine zusje zal je altijd blijven, maar eigenlijk ben je dat allang niet meer. Je houd altijd een oogje in het zeil, en vòò je het vraagt weet jij eigenlijk al hoe het met mij gaat. Ik bewonder je ondernemendheid, en je intense liefde en respect voor het leven. Ik wens je veel geluk en mooie avonturen toe samen met Alex.

Lieve papa en mama, bedankt dat jullie mij al van jongs af aan hebben gestimuleerd en gesteund om persoonlijke doelen na te streven. Dit heeft er toe geleid dat, ook als ik niet direct de beste ben, ik niet snel opgeef. Ik ben ontzettend trots als ik ook maar een beetje van jullie humor, sportiviteit, optimisme en realisme heb meegekregen. Bedankt voor de steun en het vertrouwen dat jullie mij geven bij het maken van grote of kleine beslissingen, zelfs als dat betekend dat ik wéér verder naar het zuiden ga verhuizen.

Roy, wat hadden wij een geluk toen we samen naar Maastricht konden voor onze PhD. Hoewel ons carrière-pad en passie voor het werk overeen komt, zijn wij gelukkig toch duidelijk verschillend. Met je nuchterheid, geduld en rust houd je mij in balans. Nu, na het afronden van onze PhD's staan we voor nieuwe uitdagingen. Bedankt voor het vertrouwen dat je mij geeft in jou en in mijzelf, waardoor ik deze nieuwe uitdagingen met plezier aan ga. Ik kijk uit naar onze toekomst samen. 

CURRICULUM VITAE 

Anita Kneppers was born in Valkenburg aan de Geul, the Netherlands, on June 30th, 1990. In 2007 she graduated from secondary school at the Lambert Franckens College in Elburg. In the same year, she started her Bachelor of Health Science program in Nutrition and Dietetics at HAN University of Applied Sciences, which she completed in 2011. Immediately after that, she started the Master Nutrition and Health at Wageningen University, and specialized in Molecular Nutrition and Toxicology. During this study, Anita completed a research project at the Nutrition, Metabolism and Genomics group on the molecular mechanism for reduced exercise-induced increase in expression of the myokine ANGPTL4 in active versus non-active muscle. Subsequently, she completed an internship at the Human and Animal Physiology group, during which she worked on a stable DNM1L silenced muscle cell model to study the effects on the mitochondrial morphology.

After obtaining her Master's degree in 2013, she started her PhD trajectory at the department of Respiratory Medicine at Maastricht University. This PhD project was conducted in close collaboration with the department of Human Biology, under the supervision of prof. dr. Annemie Schols, dr. Ramon Langen, dr. Lex Verdijk, and prof. dr. Luc van Loon. The primary focus of her PhD project was on the molecular processes that regulate skeletal muscle recovery after disuse-induced atrophy, with a particular focus on the role of myogenesis. Furthermore, she investigated the potential dysregulation of these processes in skeletal muscle of patients with COPD. 

LIST OF PUBLICATIONS 



\section{Published}

Kneppers A, Haast R, Langen R, Verdijk L, Leermakers P, Gosker H, van Loon L, Lainscak $\mathrm{M}$, Schols A. Distinct skeletal muscle molecular responses to pulmonary rehabilitation in COPD: a cluster analysis. J Cachexia Sarcopenia Muscle. 2018. Accepted

Kneppers A, Leermakers P, Pansters N, Backx E, Gosker H, van Loon L, Schols A, Langen R. Coordinated reguation of skeletal muscle mass and metabolic plasticity during recovery from disuse. FASEB J. 2018. [Epub ahead of print]

Leermakers P, Schols A, Kneppers A, Kelders M, de Theije C, Lainscak M*, Gosker H*. Molecular signalling towards mitochondrial breakdown is enhanced in skeletal muscle of patients with chronic obstructive pulmonary disease. Sci. Rep. 2018:8(1):15007. *shared last authorship

Ceelen J, Schols A, Kneppers A, Rosenbrand R, Drożdż M, van Hoof S, de Theije C, Kelders $M$, Verhaegen $F$, Langen R. Altered protein turnover signaling and myogenesis during impaired recovery of inflammation-induced muscle atrophy in emphysematous mice. Sci. Rep. 2018:8(1):10761.

Kneppers A, Verdijk L, de Theije C, Corten M, Gielen E, van Loon L, Schols A, Langen R. A novel in vitro model for the assessment of postnatal myonuclear accretion. Skelet Muscle. 2018:8(1):4.

Kneppers A, Langen R, Gosker H, Verdijk L, Lipovec N, Leermakers P, Kelders M, de Theije $C$, Omersa D, Lainscak $M^{*}$, Schols $A^{*}$. Increased myogenic and protein turnover signaling in skeletal muscle of COPD patients with sarcopenia. J Am Med Dir Assoc. 2017:18(7):637.e1-637.e11. *shared last authorship

Sanders $K^{*}$, Kneppers A*, van de Bool C, Langen R, Schols A. Cachexia in chronic obstructive pulmonary disease: new insights and therapeutic perspective. J Cachexia Sarcopenia Muscle. 2016;7(1):p.5-22. *shared first authorship

Catoire M, Alex S, Paraskevopulos N, Mattijssen F, Evers-van Gogh I, Schaart G, Jeppesen J, Kneppers A, Mensink M, Voshol PJ, Olivecrona G, Tan NS, Hesselink MK, Berbée JF, Rensen PC, Kalkhoven E, Schrauwen P, Kersten S. Fatty acid-inducible ANGPTL4 governs lipid metabolic response to exercise. Proc Natl Acad Sci U S A. 2014;111(11):E1043-52.

\section{Submitted for publication}

Leermakers P, Kneppers A, Schols A, Kelders M, de Theije C, Verdijk L, van Loon L, Langen $\mathrm{R}$, Gosker $\mathrm{H}$. Early-onset alterations in mitophagy and mitochondrial biogenesis signaling during skeletal muscle unloading in mice and men. Submitted. 2018. 